\title{
Monocyte Regulation By Soluble Uric Acid
}

\author{
By \\ René Joel McLaughlin
}

\author{
A thesis \\ submitted to the Victoria University of Wellington \\ in fulfilment of the requirements for a degree of \\ Doctor of Philosophy \\ in Biomedical Science
}

Victoria University of Wellington

2014 



\section{Abstract}

Hyperuricaemia is a chronic condition associated with diseases of the metabolic syndrome. However, the cause and effect relationship between increased serum uric acid (UA) levels and the pathophysiology of metabolic dysfunction is far from clear. From an immunological angle hyperuricaemia has been shown to modulate inflammatory signalling in both immune and nonimmune cell types. Blood monocytes are constantly exposed to soluble UA in the circulation but the direct effect of this exposure has not been examined. This research focuses on the how soluble UA alters blood monocyte responses to inflammatory stimuli using in vitro, in vivo and clinical manipulation of UA levels.

The Harper group previously found that blood monocytes from hyperuricaemic individuals produced lower levels of inflammatory cytokines compared to monocytes from healthy controls when stimulated ex vivo with LPS. My research began by studying the direct effect of soluble UA on human blood monocytes in vitro. I found that soluble UA reduced monocyte production of pro-inflammatory cytokines and increased IL-10 in response to stimulation with LPS. I identified two inflammatory signalling pathways modulated by soluble UA that could be contributing to this suppressive monocyte phenotype: MAP kinase phosphorylation was reduced alongside increased expression of the regulatory protein DUSP10 and reduced ASC; there was a switch towards anti-inflammatory NFKB signalling illustrated by decreased p65 and increased p50 nuclear translocation.

To study the modulation of soluble UA levels in a physiological context I raised serum UA levels in vivo with a model of acute hyperuricaemia and lowered serum UA using two clinically relevant medications: allopurinol and rasburicase. Consistent with in vitro UA treatment, raising serum UA levels in vivo suppressed pro-inflammatory cytokine responses to LPS, increased IL-10 and down-regulated monocyte MAP kinase and NFKB signalling pathways. 
Acute urate-lowering therapy (ULT) with allopurinol or rasburicase reversed this suppressive inflammatory cytokine and signalling pattern.

The PLT2 mouse strain has had the purine metabolic pathway disrupted by random mutagenesis of the gene encoding 5-hiydroxyisourate hydrolase, the enzyme responsible for degradation of the molecule directly downstream of UA, 5-hydroxyisourate. I found that this mutation resulted in chronic hyperuricaemia with an average 2-fold increase in serum UA over C57 mice. LPS challenge resulted in increased IL-10 production in PLT2 mice compared to $\mathrm{C} 57$, however no differences in monocyte inflammatory signalling were observed between the two strains. Acute ULT with rasburicase reduced serum UA in the PLT2 strain and subsequent LPS challenge increased monocyte inflammatory signalling.

Finally, I studied the effects of ULT on the inflammatory phenotype of human blood monocytes from patients with hyperuricaemia. ULT significantly reduced serum UA levels, which coincided with reduced blood monocyte percentages and adhesion molecule expression (CD11b and ICAM1). ULT increased the inflammatory potential of human blood monocytes: Monocytes stimulated with LPS produced less IL-10; MAP kinase phosphorylation increased alongside increased ASC expression; nuclear translocation of NFкB p65 was increased. ULT also increased expression of the NLRP3 inflammasome components procaspase1, pro-IL-1 $\beta$ and NLRP3.

Taken together these results demonstrate a previously unidentified role for soluble UA in moderating monocyte immune responses to inflammatory stimuli. In vitro, in vivo and clinical experimentation all confirmed the immunosuppressive function of soluble UA. This potentially places UA in the centre of innate immune control through the dichotomy of its suppressive soluble effects, demonstrated herein, and the widely reported inflammatory crystalline effects. Importantly, this research illustrates that serum UA levels can be manipulated in a clinical setting to control the inflammatory phenotype of circulating immune cells. 


\section{Acknowledgements}

To make this a little easier on the reader I am going to split this section into two. That way if you've been a part of my scientific family you'll want to look for your name in the first half and if you are part of my more conventional family (friends are included here as family) you can skip the first two pages and start skimming from the third.

I hope that if you are reading this and expect to find yourself listed here that I haven't somehow forgotten you but if I have I apologise in advance and you can give me an earful later.

To begin with I would like to thank my super-visor (pun definitely intended) Jacquie Harper. She was instrumental, not only during this $\mathrm{PhD}$, but in bringing me into the world of immunology. She gave a naïve undergrad a taste of the lab life and opened up a world of immunological horizons that were previously unknown to him. I admire her for her candour and no holds barred attitude to science and would like to think l've managed to absorb some of her knowledge and wisdom during our time together. Only time will tell...

The team Jacquie brought together was nothing but exceptional. Steffi, my $\mathrm{PhD}$ sister, it was wonderful struggling along beside you and happily we were able to fall across the finish line together. Odette was the rock that held us all together and to complement her many casual threats of physical harm (usually stabbing) she was an invaluable source of technical know-how and comic relief. Lisa was the best lab buddy a guy could hope for. We developed a solid disdain for $\mathrm{Ke}$ (dollar sign)ha together but also knew when another favourite pop hit was brewing that we could both sing along to. I was so happy when you got that golden opportunity in Sydney but you were missed very much. 
To the other group members who have moved on, Willy-John, Shujie, Mischa and even going back to Liz and Oliver. You have all had a positive influence on my immunological development and deserve a special mention.

The Malaghan PhD club is a fantastic little group of hardworking, idealistic and supportive students all just trying to survive the process but at the same time managing to pass on a little of their hard earned wisdom to the next generation. To the generation before me, my contemporaries and the ones still wet behind the ears: thank you for the camaraderie.

Of course an immunologist is not a one-man-band and without the whole Malaghan family behind me none of this would have been possible. I would especially like to thank the tireless work of the support staff who keep the well-oiled machine running full steam ahead and Janine for the lunchtime crossword sessions; they were a wonderful distraction from the all-consuming lab work.

My final experimental chapter I owe to the wonderful people in Christchurch. Lisa generously offered me patients when I had none of my own to work with. Janine organised them all for me and made sure they came in on time and gave me lots of precious blood. Tony gave me a lab to work in and his team answered my every (sometimes naïve) question and became my temporary science family for a few months.

Donia and Miles I would like to credit as the font of all statistical knowledge. Dealing with my clinical data was difficult but their insight and enthusiasm for large data sets made it all possible and even fun.

I have come to appreciate that good science requires adequate funding and as such would like to say a big thank you to all those who contributed to my research; Arthritis NZ, JL Stewart, Wellington Medical Research Foundation and the Maurice and Phyllis Paykel Trust and those that supported me and the Malaghan through donations when I ran for research or shook a bucket during the lollipop appeal. 
A PhD is a personal as well as a scientific experience and I think the role of my friends and family in this process cannot be overestimated.

My folks deserve a special mention, not just for having me, but also for their limitless support and for keeping us supplied with delicious home-grown fruit and veg and good quality cheese. So cheers guys, thanks a lot.

Ultimate Frisbee is the great provider of everything good in life; friends, fun, fitness, fresh air, travel, distraction... the list goes on. So to all the wonderful people (there are too many to name) in Wellington, in NZ and around the world who have provided Ultimate relief I credit you with my sanity and wish you all many happy years of chasing plastic.

Near the end I relied heavily on the unlimited generosity of Ellie and Todd who put me up, fed me and kept me warm during my many and frequent visits to Christchurch. You guys are the best and I wish there was some way I could fully repay you.

I devote this final paragraph to my devoted wife. I can honestly credit her with my success, not just in this PhD but also in life in general. She was the one who at the beginning of it all said, "Hey, you like biology don't you? Why don't you come to Uni with me and study biology?" Then she was there beside me doing the hard yards and long (last minute) hours of undergrad and honours. Finally, she was the one who kept me functioning during the long haul to the finish line. I can't wait to return the favour during your PhD BB. 


\section{Table of Contents}

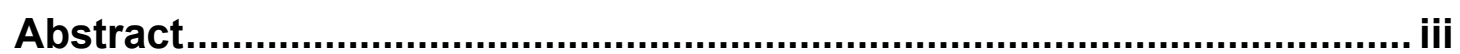

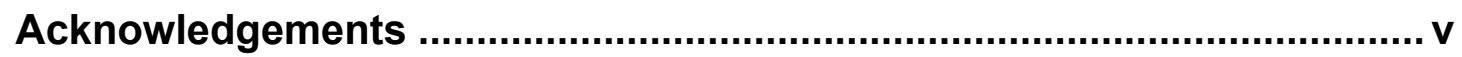

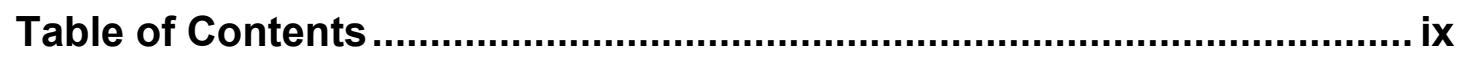

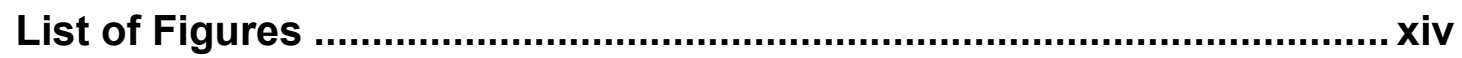

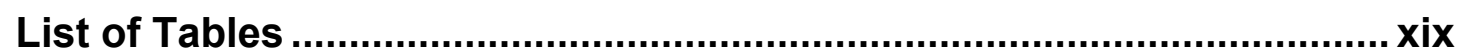

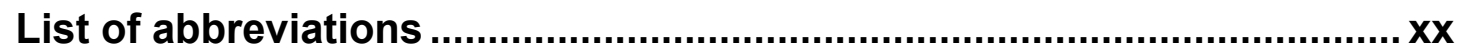

1 General introduction ....................................................................... 1

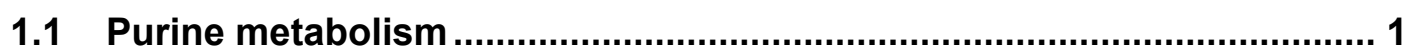

1.2 Evolutionary mutational silencing of uricase ......................................... 1

1.3 Dysregulation of UA homeostasis......................................................... 4

1.3.1 Hypouricaemia (serum UA $<0.12 \mathrm{mM}$ or $<2 \mathrm{mg} / \mathrm{dl}$ ) ….......................... 4

1.3.2 Risk factors for hyperuricaemia (serum $U A>0.42 \mathrm{mM}$ or $>7 \mathrm{mg} / \mathrm{dl}$ )......... 4

1.4 Diseases associated with UA dysregulation ........................................... 7

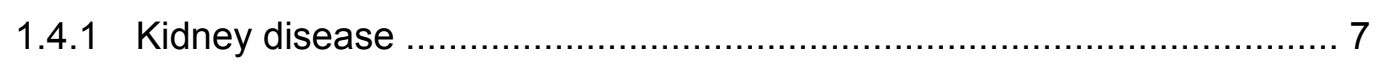

1.4.2 Hypertension and cardiovascular disease .......................................... 7

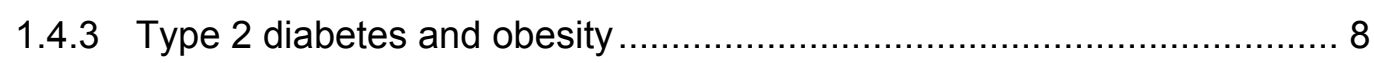

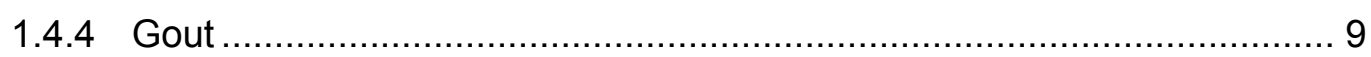

1.5 Treatment of hyperuricaemia ............................................................. 10

1.5.2 Xanthine oxidoreductase (XOR) inhibition ....................................... 11

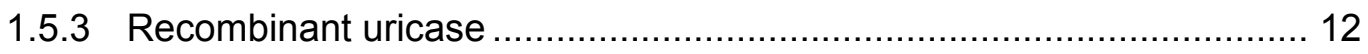

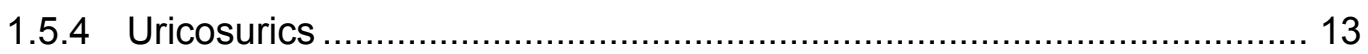

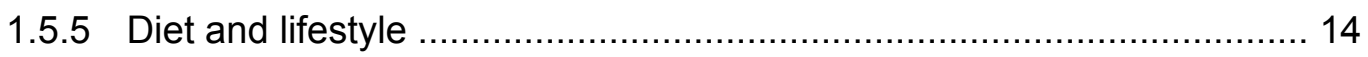

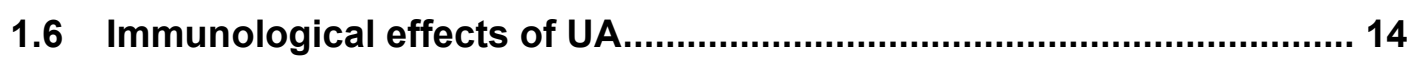

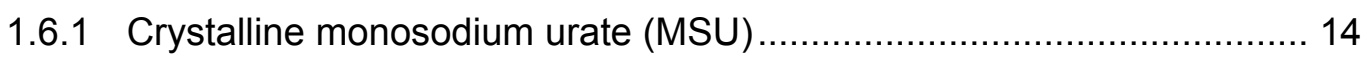

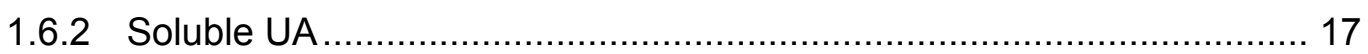

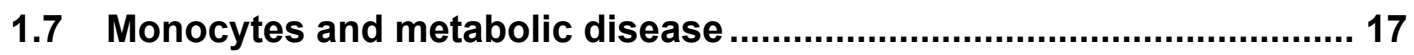

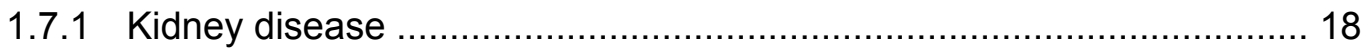

1.7.2 Hypertension and cardiovascular disease ........................................ 19

1.7.3 Type 2 diabetes and obesity ………................................................ 19

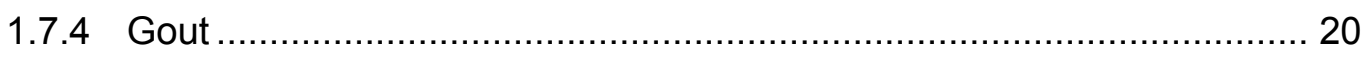

1.8 Experimental hyperuricaemia ......................................................... 20 


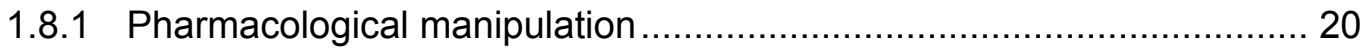

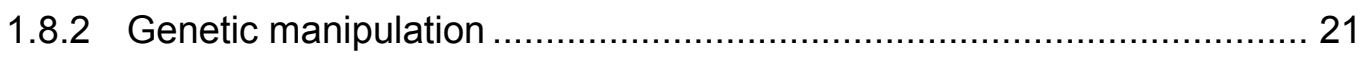

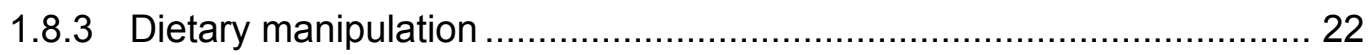

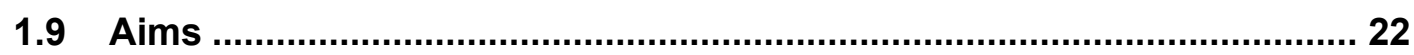

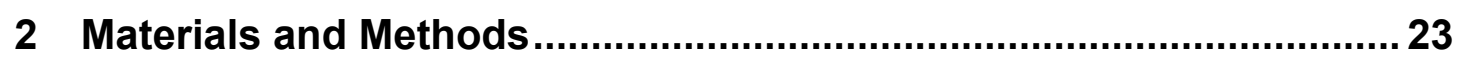

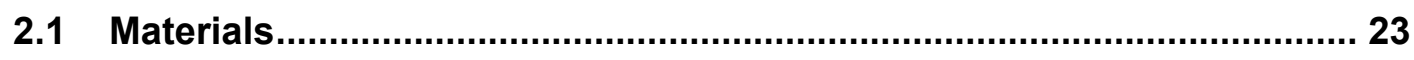

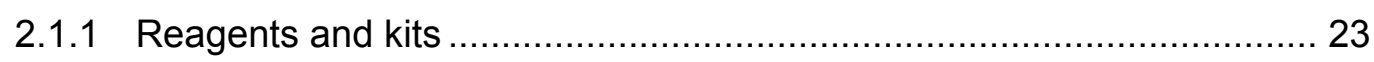

2.1 .2 Antibodies .......................................................................... 25

2.1.3 Commercial buffers and media .................................................. 26

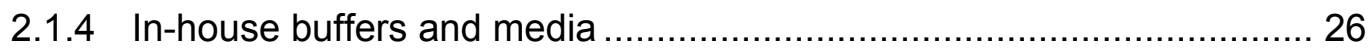

2.1.5 Preparation of monosodium urate (MSU) crystals .............................. 28

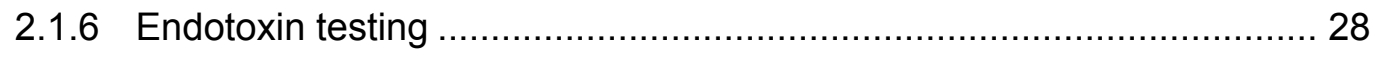

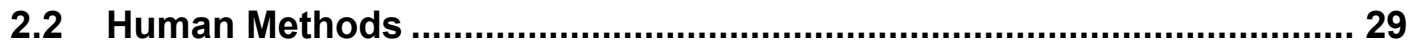

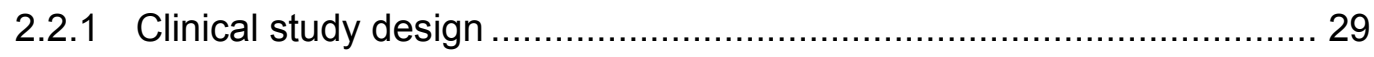

2.2 .2 Isolation of human blood monocytes .............................................. 29

2.2 .3 In vitro monocyte activation assays ............................................. 30

2.2.4 Monocyte photography and cluster analysis.................................. 30

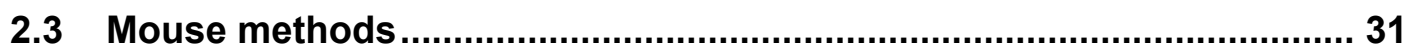

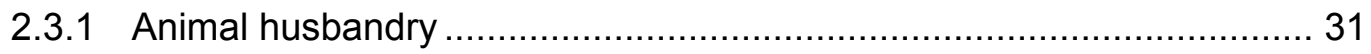

2.3.2 Mouse model of acute hyperuricaemia ............................................. 31

2.3.3 Mouse models of urate-lowering therapy (ULT) ............................... 31

2.3.4 LPS model of systemic inflammation............................................... 32

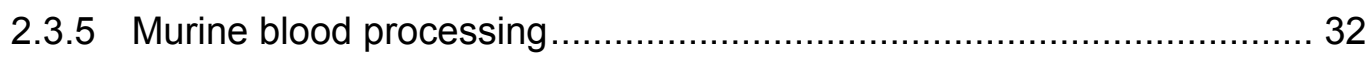

2.3.6 Murine spleen processing ........................................................ 32

2.3.7 AutoMACS purification of splenic monocytes ................................. 32

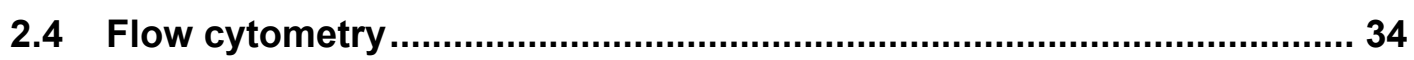

2.4.1 Surface staining for flow cytometry .................................................... 34

2.4.2 Intracellular staining for flow cytometry ......................................... 34

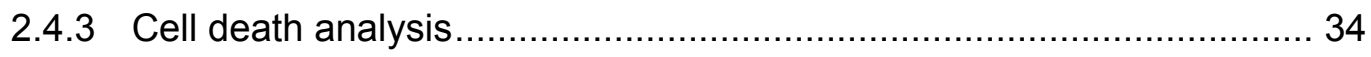

2.4.4 Intracellular reactive oxygen species .......................................... 34

2.4.5 Flow cytometry equipment .......................................................... 35

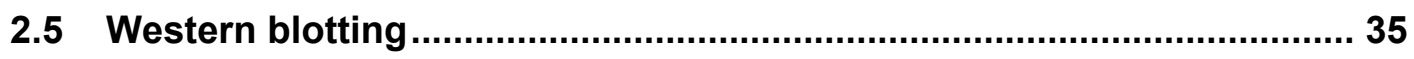

2.5.1 Cytosolic and nuclear protein isolation .......................................... 35

2.5.2 Determining protein concentration .................................................. 35

2.5.3 Gel electrophoresis .................................................................. 36

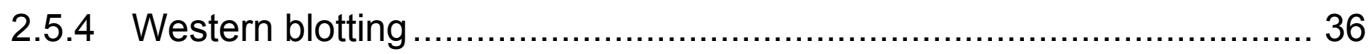




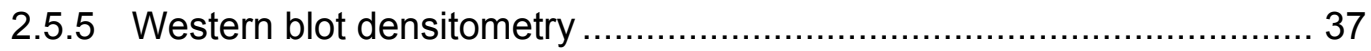

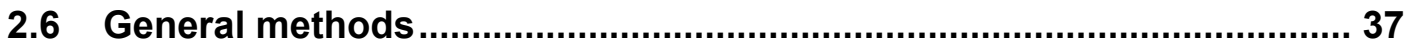

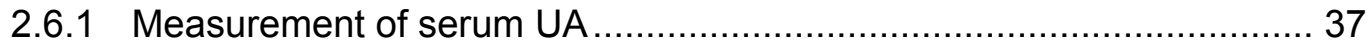

2.6.2 Chemical assay for anti-oxidant activity............................................... 38

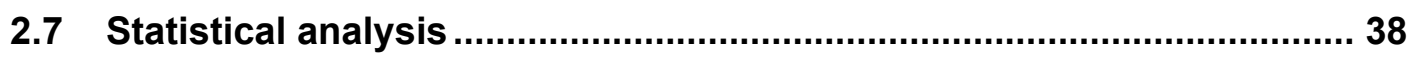

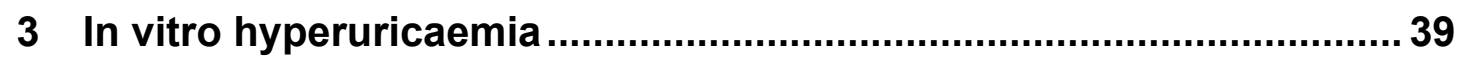

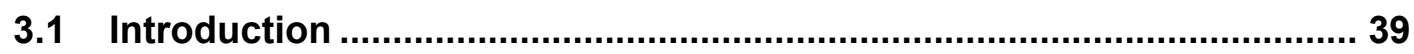

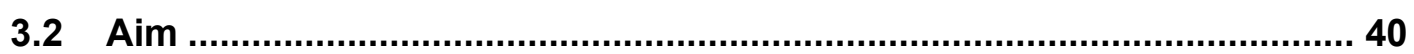

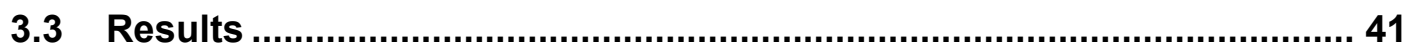

3.3.1 Monocyte viability and soluble UA …............................................ 41

3.3.2 Monocyte cytokine production .................................................... 42

3.3.3 Monocyte LPS signalling machinery .............................................. 42

3.3.4 Intracellular reactive oxygen species (ROS) generation during LPS

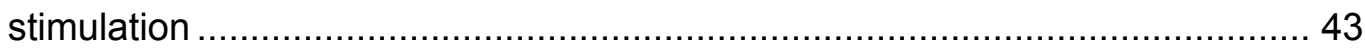

3.3.5 Testing UA as an electron scavenger ........................................ 44

3.3.6 NFKB signalling pathway ............................................................. 45

3.3.7 Anti-inflammatory cytokine production ......................................... 48

3.3.8 Involvement of MAP kinases and their regulation by UA ..................... 48

3.3.9 Adenosine receptors and purine recognition ................................... 51

3.3.10 The role of adenosine receptors in UA-enhanced monocyte IL-10

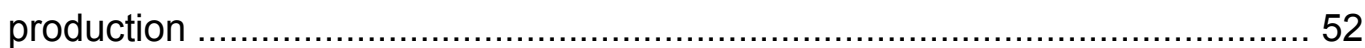

3.3.11 A2a and A2b receptor signalling and protein kinase $A(P K A)$........... 55

3.3.12 Monocyte homotypic aggregation ............................................. 56

3.3.13 Monocyte aggregation and adenosine receptor activation ................ 57

3.3.14 Monocyte adhesion molecule expression .......................................... 62

3.3.15 Monocyte chemokine production ................................................. 64

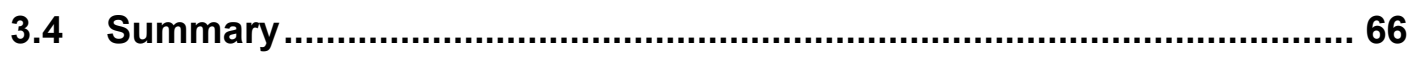

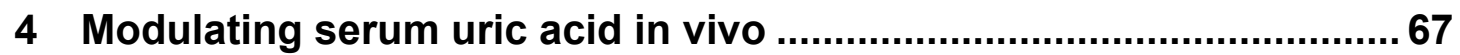

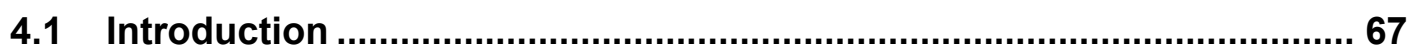

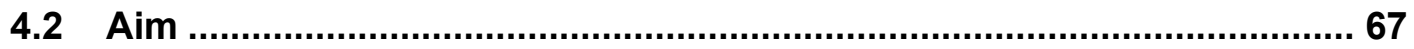

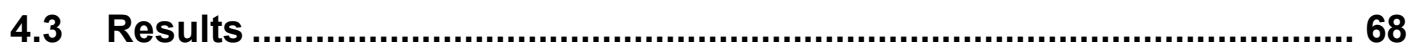

4.3.1 A mouse model of acute hyperuricaemia .......................................... 68

4.3.2 Profiling the systemic cytokine response to LPS during acute

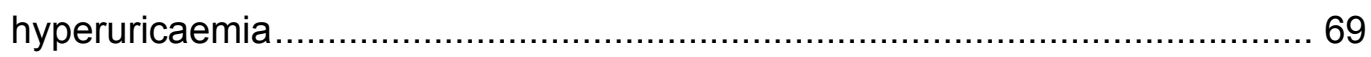

4.3.3 The in vivo cellular response to acute hyperuricaemia and LPS .......... 70

4.3.4 Lowering serum UA in vivo .................................................... 74 
4.3.5 A mouse model of chronic hyperuricaemia .................................... 80

4.3.6 Lowering serum UA in chronic hyperuricaemia .............................. 84

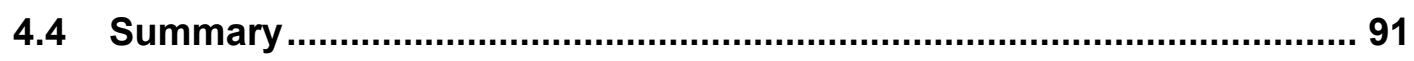

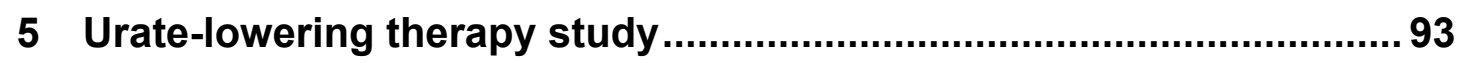

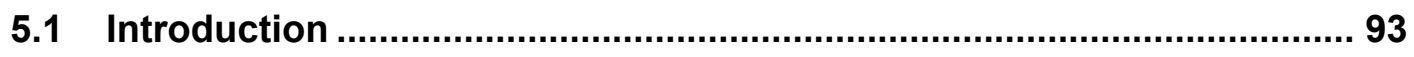

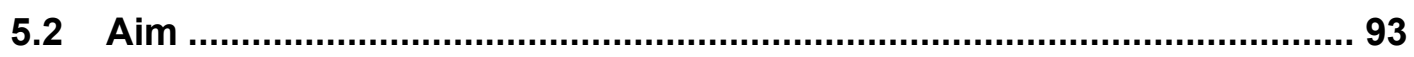

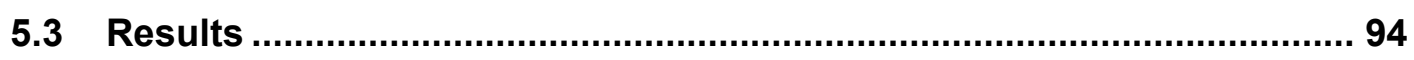

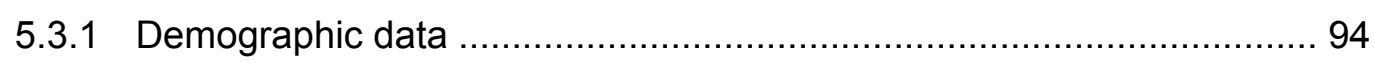

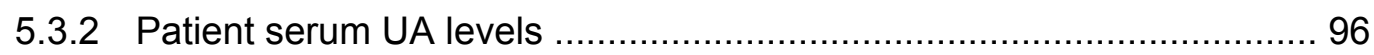

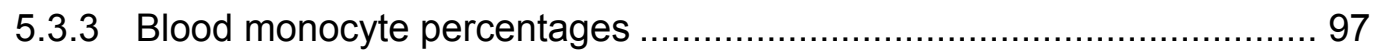

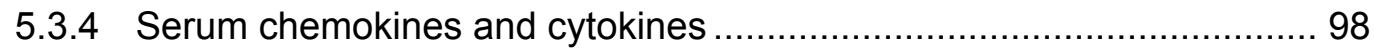

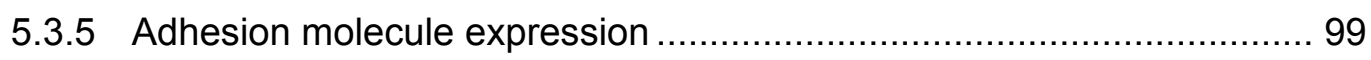

5.3.6 Monocyte cytokine production following ULT ................................ 102

5.3.7 Monocyte chemokine production following ULT .............................. 107

5.3.8 ULT and cytosolic monocyte MAP kinase signalling ........................ 111

5.3.9 ULT and monocyte protein expression in the nucleus ...................... 116

5.3.10 Monocyte inflammasome signalling following ULT ........................ 118

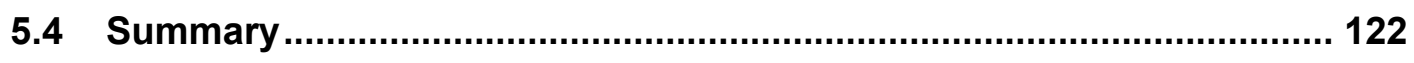

6 Discussion and future directions.................................................... 124

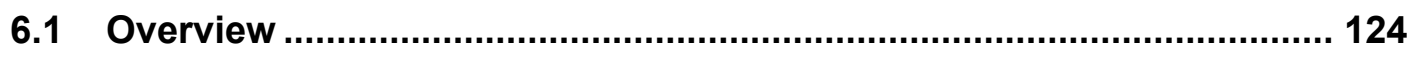

6.2 Monocyte inflammatory signalling and cytokine production .............. 125

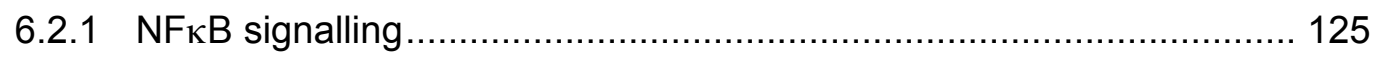

6.2.2 MAP kinase signalling ......................................................... 127

6.2.3 NLRP3 inflammasome signalling ............................................... 128

6.3 Monocyte adhesion molecule expression and CCL2 ......................... 129

6.4 Murine hyperuricaemia and urate-lowering therapy........................... 131

6.4.1 Acute hyperuricaemia and ULT with allopurinol and rasburicase ........ 131

6.4.2 The PLT2 mouse strain as a model for chronic hyperuricaemia ......... 132

\subsection{Modulating soluble UA as a method of controlling inflammation and}

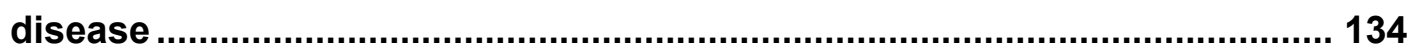

6.5.1 Hyperuricaemia and sepsis .................................................. 134

6.5.2 Hyperuricaemia and metabolic syndrome ..................................... 135

6.5.3 Soluble versus crystalline uric acid ............................................ 136

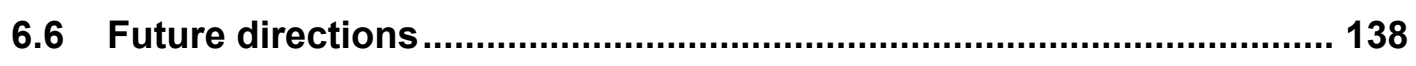

6.6.1 Immune cell signalling ....................................................... 138

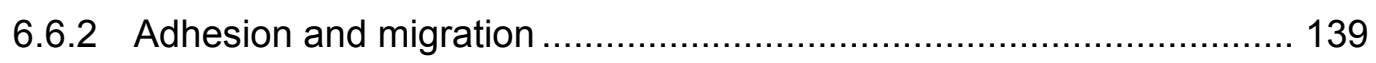


6.6.3 Murine hyperuricaemia and urate-lowering therapy 140

6.6.4 Human urate-lowering therapy studies

References

7 Appendices 156

7.1 NFKB signalling in human monocytes following in vitro UA treatment 156

7.2 MAP kinase signalling in human monocytes following in vitro UA treatment.

7.3 Densitometric analysis of western blot data from figure 4.5 and 4.6.. 158

7.4 Densitometric analysis of western blot data from figure 4.8. 159

7.5 Densitometric analysis of western blot data from figure 4.10 160

7.6 Densitometric analysis of western blot data from figure 4.11 161

7.7 Densitometric analysis of western blot data from figure 4.14 and 4.15 162

7.8 Densitometric analysis of western blot data from figure 4.17 and 4.18 163

7.9 Densitometric analysis of western blot data from figure 4.20 and 4.21

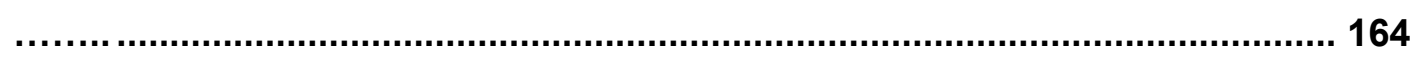

7.10 Serum cytokines and chemokines during ULT ................................ 165

7.11 Serum cytokines and chemokines versus serum UA levels ............. 166

7.12 Clinical western blots of LPS treated monocytes............................. 167

7.13 Clinical western blots of MSU treated monocytes............................ 168 


\section{List of Figures}

Figure 1.1 Purine metabolic pathway.

Figure 1.2 Different mechanisms of pharmacological urate-lowering therapy.

Figure 1.3 Adjuvanticity of MSU.

Figure 2.1 Mouse models of acute hyperuricaemia, urate-lowering therapy and systemic inflammation.

Figure 3.1 Ex vivo monocyte cytokine production negatively correlates with serum UA levels. 40

Figure 3.2 Monocyte viability is unaffected by UA.

Figure 3.3 UA reduces LPS-induced pro-inflammatory cytokines and enhances IL-10.

Figure 3.4 CD14 and TLR4 expression is unaffected by UA.

Figure 3.5 Monocyte intracellular ROS is unaffected by UA. 44

Figure 3.6 Soluble UA is not an electron scavenger. 45

Figure 3.7 LPS-induced monocyte pro- and anti-inflammatory cytokine production via NFKB. 46

Figure 3.8 IKB $\alpha$ levels return faster with UA treatment after LPS stimulation.

Figure 3.9 UA enhances LPS-induced IL-10 and reduces IL-12p40...... 48

Figure 3.10 LPS-induced monocyte pro-inflammatory cytokine production via MAP kinase signalling.

Figure 3.11 UA treatment reduces ASC and increases DUSP10 expression.

Figure 3.12 Chemical structures of uric acid, adenosine and caffeine highlighting their core purine structure.

Figure 3.13 A2a receptor antagonist inhibits the IL-10 increase caused by UA. 53

Figure 3.14 UA increases monocyte IL-10 independently of adenosine and $A 2$ a receptor signalling.

Figure 3.15 UA does not signal through the A2a or A2b receptor........ 56

Figure 3.16 Monocyte aggregation is enhanced by UA. 
Figure 3.17 Monocyte aggregation is inhibited by the A2a receptor antagonist.

Figure 3.18 Monocyte aggregation, but not cluster formation, is inhibited by the $\mathrm{A} 2 \mathrm{a}$ receptor antagonist.

Figure 3.19 UA enhances LPS-induced aggregation independently of adenosine signalling.

Figure 3.20 UA enhances LPS-induced aggregation independently of adenosine signalling.

Figure 3.21 Monocyte adhesion molecule expression is unchanged by UA or adenosine.

Figure 3.22 Monocyte chemokine production is not affected by UA....65

Figure 4.1 OA and UA induce acute hyperuricaemia in C57 mice. .......68

Figure 4.2 IL-10 peaks in mouse serum 1 hour after LPS challenge. ... 69

Figure 4.3 In vivo hyperuricaemia reduces inflammatory cytokines and increases IL-10 in response to LPS. 70

Figure 4.4 A population of splenic CD11b+/Ly6C+ monocytes produces IL-10 upon LPS challenge and increases in frequency in hyperuricaemic mice.

Figure 4.5 Hyperuricaemia reduces ASC expression and MAP kinase phosphorylation in splenic monocytes and decreases NFKB p65 nuclear translocation.

Figure 4.6 Hyperuricaemia increases pro-caspase1 expression and decreases active IL-1 $\beta$ in splenic monocytes.

Figure 4.7 Allopurinol treatment lowers serum UA and increases LPSinduced systemic inflammation. .75

Figure 4.8 Allopurinol treatment reduces monocyte $\mathrm{I}_{\kappa} \mathrm{B} \alpha$ expression and increases NFKB p65 nuclear translocation.

Figure 4.9 Rasburicase treatment lowers serum UA but does not alter LPS-induced systemic inflammation.

Figure 4.10 Rasburicase treatment alone increases monocyte ASC expression and NFKB nuclear translocation. .78

Figure 4.11 ULT with rasburicase increases NLRP3 but decreases procaspase 1 and pro-IL-1 $\beta$ expression in splenic monocytes. 
Figure 4.12 Mouse purine metabolic pathway from hypoxanthine to allantoin.

Figure 4.13 Serum UA and cytokine levels are higher in LPS treated PLT2 mice compared with C57 mice.

Figure 4.14 Chronic hyperuricaemia in PLT2 mice does not affect monocyte inflammatory signalling molecules with or without LPS challenge.

Figure 4.15 After LPS challenge PLT2 mouse monocytes have decreased NLRP3 and pro-IL-1 $\beta$ expression compared to C57.

Figure 4.16 Allopurinol treatment has no effect on either serum UA or LPS-induced serum cytokines in PLT2 mice. 85

Figure 4.17 Allopurinol treatment has no effect on ASC expression in splenic monocytes from PLT2 mice.

Figure 4.18 Allopurinol treatment increases NLRP3 expression in splenic monocytes from PLT2 mice.

Figure 4.19 Rasburicase treatment lowers serum UA but has no effect on LPS-induced systemic inflammation in PLT2 mice. 88

Figure 4.20 Rasburicase treatment increases $p$-ERK1/2 and increases NFKB p65 subunit nuclear translocation in splenic monocytes from PLT2 mice. 89

Figure 4.21 Rasburicase treatment increases pro-caspase1 and pro-IL$1 \beta$ expression in splenic monocytes from PLT2 mice. 90

Figure 5.1 ULT reduces patient serum UA levels. .96

Figure 5.2 Representative flow plot of CD14 and CD16 stained white blood cells (WBC).

Figure 5.3 Monocyte and neutrophil percentages before and after ULT.

Figure 5.4 There is no relationship between ULT or serum UA levels and serum CCL2.

Figure 5.5 Monocyte and neutrophil CD11b expression decreases with ULT. 100

Figure 5.6 ULT reduces expression of monocyte adhesion molecules. 
Figure 5.7 ULT reduces LPS-induced monocyte IL-10 production. .... 102

Figure 5.8 Correlation analysis of monocyte IL-10 and IL12p40

production versus serum UA levels. 103

Figure 5.9 ULT does not affect monocyte TNF $\alpha$ or IL-6 production. .. 104

Figure 5.10 Serum UA negatively correlates with MSU-induced monocyte TNF $\alpha$ and IL-6 production. 104

Figure 5.11 MSU-induced monocyte IL-1 $\beta$ production is reduced following ULT. 105

Figure 5.12 Correlation analysis of monocyte IL-1 $\beta$ and IL-1Ra production compared to serum UA. 106

Figure 5.13 MSU-induced monocyte CCL2 production increases following ULT.

Figure 5.14 Correlation analysis of monocyte IL-8 and CCL2 production compared to serum UA. 108

Figure 5.15 MSU-induced monocyte CCL7 production increases following ULT. 109

Figure 5.16 Correlation analysis of monocyte CCL4, CCL5 and CCL7 production compared to serum UA.

Figure 5.17 ULT increases monocyte MAP kinase phosphorylation and ASC expression.

Figure 5.18 ULT increases monocyte MAP kinase phosphorylation and ASC expression. 113

Figure 5.19 ULT increases monocyte DUSP1 phosphorylation. 114

Figure 5.20 ULT increases monocyte DUSP1 phosphorylation. 115

Figure 5.21 ULT increases monocyte NFKB p65 nuclear translocation and decreases nuclear p50.

Figure 5.22 ULT increases NFKB nuclear translocation in LPSstimulated monocytes but decreases ASC and Bcl3 in MSUstimulated monocytes.

Figure 5.23 ULT increases monocyte NLRP3 inflammasome components.

Figure 5.24 ULT increases monocyte NLRP3 inflammasome components. 
Figure 6.1 Potential mechanisms through which soluble UA suppresses monocyte inflammatory signalling. 126

Figure 6.2 Proposed model of soluble UA controlling low-level inflammation and the switch to crystalline UA (MSU) activating innate and adaptive immunity during severe inflammation.

Figure 7.1 NFKB p65 nuclear translocation is inhibited and p50 in enhanced by soluble UA. 156

Figure 7.2 p38 and ERK1/2 MAP kinases are more rapidly dephosphorylated in UA treated monocytes.

Figure 7.3 Densitometric analysis of western blot data from figure 4.5 and 4.6. 158

Figure 7.4 Densitometric analysis of western blot data from figure 4.8. 159

Figure 7.5 Densitometric analysis of western blot data from figure 4.10. 160

Figure 7.6 Densitometric analysis of western blot data from figure 4.11.

Figure 7.7 Densitometric analysis of western blot data from figure 4.14 and 4.15.

Figure 7.8 Densitometric analysis of western blot data from figure 4.17 and 4.18.

Figure 7.9 Densitometric analysis of western blot data from figure 4.20 and 4.21 .

Figure 7.10 ULT does not alter human serum cytokine or chemokine levels.

Figure 7.11 Serum IL-10 and IL-8 levels negatively correlate with serum UA. 166

Figure 7.12 Western blots of LPS treated monocyte cytosolic protein from patients undergoing ULT.

Figure 7.13 Western blots of MSU treated monocyte cytosolic protein from patients undergoing ULT. 


\section{List of Tables}

Table 5.1 Serum UA levels, demographic data, comorbidities and medication. 


\section{List of abbreviations}

\begin{tabular}{|l|l|}
\hline Ad & Adenosine \\
\hline ADA & Adenosine deaminase \\
\hline ahs & Autologous human serum \\
\hline ANOVA & Analysis of variance \\
\hline APC & Allophycocyanin \\
\hline ASC & Apoptosis associated speck-like protein containing a CARD \\
\hline ATP & Adenosine triphosphate \\
\hline Bcl3 & B cell lymphoma 3 \\
\hline BMI & Body mass index \\
\hline BSA & Bovine serum albumin \\
\hline C57 & C57BL/6 \\
\hline CCL2 & Chemokine (C-C motif) ligand 2 \\
\hline CCL3 & Chemokine (C-C motif) ligand 5 \\
\hline CCL4 & Chemokine (C-C motif) ligand 4 \\
\hline CCL5 & Chemokine (C-C motif) ligand 5 \\
\hline CCL7 & Chemokine (C-C motif) ligand 7 \\
\hline CCR2 & Chemokine (C-C motif) receptor 2 \\
\hline CD11b & Cluster of differentiation 11b \\
\hline CD14 & Cluster of differentiation 14 \\
\hline CD16 & Cluster of differentiation 16 \\
\hline CD162 & Cluster of differentiation 162 \\
\hline CD3 & Cluster of differentiation 3 \\
\hline CD8 & Cluster of differentiation 8 \\
\hline CD31 & Cluster of differentiation 31 \\
\hline CD62L & Cluster of differentiation 62L \\
\hline CPPD & Calcium pyrophosphate dihydrate \\
\hline DAMP & Damage associated molecular pattern \\
\hline DC & Dendritic cell \\
\hline DHR-123 & Dihidrorhodamine-123 \\
\hline & \\
\hline
\end{tabular}




\begin{tabular}{|l|l|}
\hline DMSO & Dimethyl sulfoxide \\
\hline DOCK2 & Dedicator of cytokinesis 2 \\
\hline DPBS & Dulbecco's phosphate buffered saline \\
\hline dsRNA & Double stranded ribonucleic acid \\
\hline DUSP1 & Dual specific phosphatase 1 \\
\hline DUSP10 & Dual specific phosphatase 10 \\
\hline EDTA & Ethylenediaminetetraacetic acid \\
\hline EGCG & Epigallocatechin gallate \\
\hline ELISA & Enzyme-linked immunosorbent assay \\
\hline ERK & Extracellular signal-regulated kinase \\
\hline FACS & Fluorescence-activated cell sorting \\
\hline FBS & Foetal bovine serum \\
\hline FITC & Fluorescein isothiocyanate \\
\hline HBSS & Hank's balanced salt solution \\
\hline HEPES & 2-[4-(2-hydroxyethyl)piperazin-1-yl]ethanesulfonic acid \\
\hline HIU & 5-hydroxyisourate \\
\hline ICAM1 & Intracellular adhesion molecule 1 \\
\hline IL-10 & Interleukin-10 \\
\hline IL-12p40 & Interleukin-12p40 \\
\hline IL-1Ra & Interleukin-1 receptor antagonist \\
\hline IL-1 $\beta$ & Interleukin-1 $\beta$ \\
\hline IL-6 & Interleukin-6 \\
\hline IL-8 & Interleukin-8 \\
\hline IKB $\alpha$ & Inhibitor of NFKB $\alpha$ \\
\hline LPS & Lipopolysaccharide \\
\hline MACS & Magnetic-activated cell sorting \\
\hline MAP kinase & Mitogen activated protein kinase \\
\hline MES & 2-(N-morpholino)ethanesulfonic acid \\
\hline MFI & Mean fluorescent intensity \\
\hline mPMS & mothoxy-5-methylphenazinium methylsulfate \\
\hline MSU & \\
\hline NADH & -Nium urate \\
\hline
\end{tabular}




\begin{tabular}{|c|c|}
\hline NFKB & Nuclear factor $\kappa \mathrm{B}$ \\
\hline NLRP3 & NACHT, LRR and PYD domain-containing protein 3 \\
\hline NSAID & Non-steroidal anti-inflammatory drug \\
\hline $\mathrm{OA}$ & Oxonic acid \\
\hline $\mathrm{OHCU}$ & 2-oxo-4-hydroxy-4-carboxy-5-ureidoimidazoline \\
\hline PAGE & Polyacrylamide gel electrophoresis \\
\hline PAMP & Pathogen associated molecular pattern \\
\hline PBMC & Peripheral blood mononuclear cell \\
\hline PBS & Phosphate buffered saline \\
\hline $\mathrm{PE}$ & Phycoerythrin \\
\hline PerCP & Peridinin chlorophyll protein \\
\hline PKA & Protein kinase $\mathrm{A}$ \\
\hline PLT2 & $U_{r a h}{ }^{P t 2 / P I t 2}$ \\
\hline PMA & Phorbol myristate acetate \\
\hline PMSF & Phenylmethylsulfonyl fluoride \\
\hline PVDF & Polyvinyl difluoride \\
\hline RBC & Red blood cell \\
\hline ROS & Reactive oxygen species \\
\hline RPMI & Roswell park memorial institute medium \\
\hline sf & Serum free \\
\hline shRNA & Short hairpin ribonucleic acid \\
\hline SOD & Superoxide dismutase \\
\hline SUMO & Small ubiquitin-like modifier \\
\hline TBP & Tata binding protein \\
\hline TLR4 & Toll-like receptor 4 \\
\hline TNF $\alpha$ & Tumour necrosis factor $\alpha$ \\
\hline UA & Uric acid \\
\hline ULT & Urate-lowering therapy \\
\hline WBC & White blood cell \\
\hline WST-1 & $\begin{array}{l}\text { 2-(4-lodophenyl)-3-(4-nitrophenyl)-5-(2,4-disulfophenyl)-2H- } \\
\text { tetrazolium }\end{array}$ \\
\hline XOR & Xanthine oxidoreductase \\
\hline
\end{tabular}


Chapter 1:

General Introduction 



\section{General introduction}

Elevated serum uric acid (UA) was first identified in patients with gout by Alfred Baring Garrod in $1848^{1}$. We have now come to recognise that soluble uric acid (UA) plays a multifaceted role in a multitude of inflammatory diseases including metabolic syndrome and neurological disorders. However, it is still unclear whether hyperuricaemia $(>0.42 \mathrm{mM}$ serum UA) is protective or contributes to metabolic disorders such as heart disease, obesity and type 2 diabetes $^{2}$. Furthermore, there is a paucity of information regarding the direct effect of soluble UA on immune cell function during inflammation and disease. Identifying how soluble UA interacts with immune cells could provide important insights into the pathophysiology of hyperuricaemia in the context of metabolic disorders and the role soluble UA plays in homeostasis of the immune system.

\subsection{Purine metabolism}

The purine metabolic pathway describes the generation and breakdown of nucleotides for DNA synthesis, namely adenosine and guanosine. Uric acid (UA) is the end product of purine degradation in humans, whereas in most mammals allantoin is the end product (figure 1.1). Adenosine and guanosine are broken down through a series of enzymatic steps into hypoxanthine and xanthine respectively ${ }^{3}$. From this point a single enzyme, xanthine oxidoreductase (XOR), catalyses the production of UA. In most mammals UA is then oxidised by uricase into 5-hydroxyisourate (HIU) and two more enzymatic steps catabolise HIU into the end product allantoin, which is then excreted via the kidneys and urine ${ }^{4}$.

\subsection{Evolutionary mutational silencing of uricase}

During our common evolution, humans and higher primates acquired a series of mutations in the uricase gene that has resulted in a complete loss of function ${ }^{5}$. These mutations have been traced back to different periods of species divergence in the hominids and great apes. 


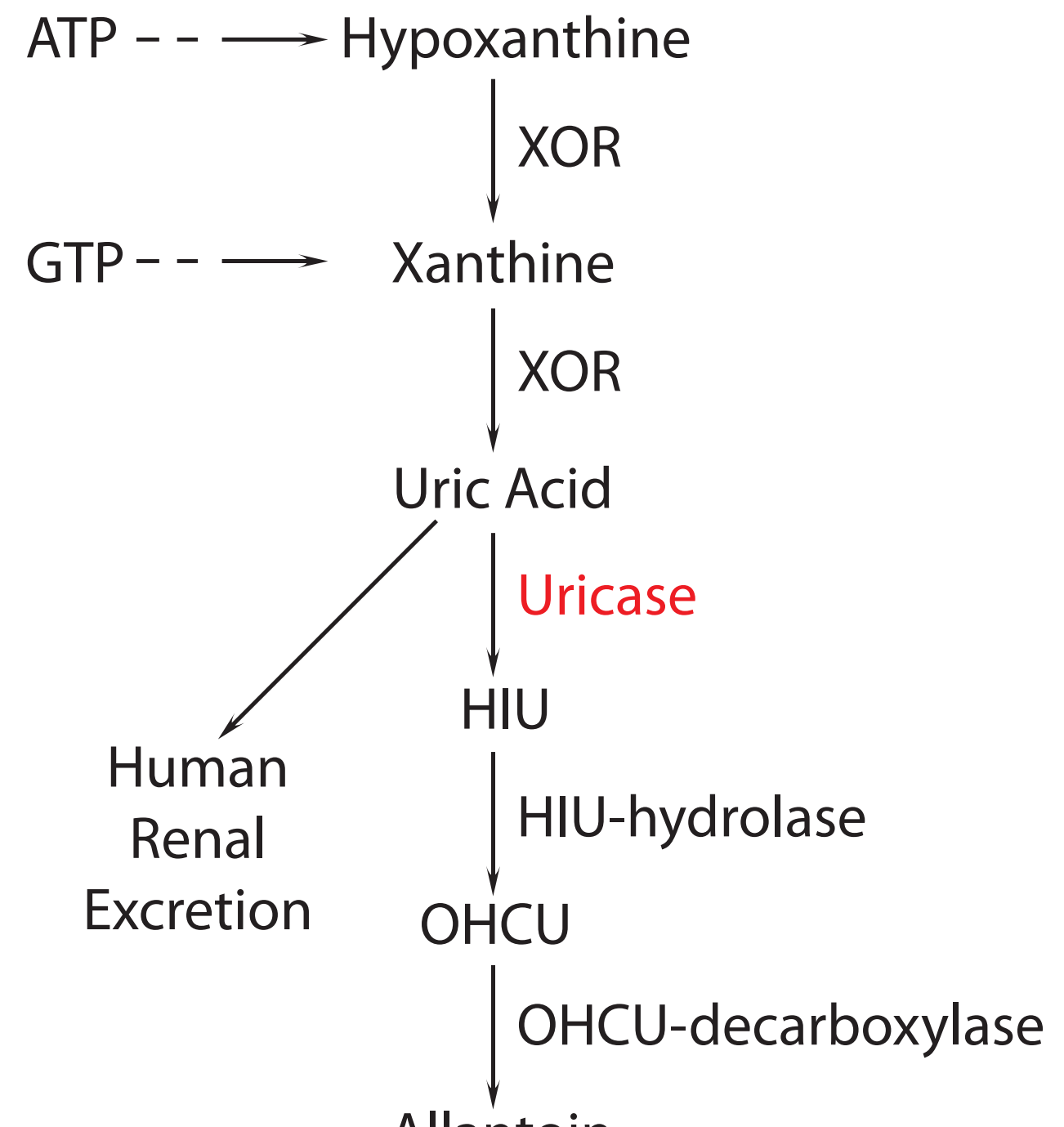

Allantoin

Figure 1.1 Purine metabolic pathway.

Humans no longer have a functional uricase enzyme. This makes uric acid the endpoint of purine degradation in humans. ATP: Adenosine triphosphate, GTP: Guanosine triphosphate, XOR: Xanthine oxidoreductase, HIU: 5-hydroxyisourate, OHCU: 2-oxo-4-hydroxy-4-carboxy-5-ureidoimidazoline. 
Combined with the evolutionary loss of uricase is the efficient retention of UA in humans by reabsorption via UA transporters in the kidneys, such that about $90 \%$ of the UA that is filtered through the kidneys is reabsorbed into the blood $^{6}$. This contributes to average serum UA levels in humans of around $0.36 \mathrm{mM}(6 \mathrm{mg} / \mathrm{dl}), 10$-fold higher than most other mammals.

There are several theories regarding the drivers of evolutionary selection for a loss of uricase function and increased serum UA.

Firstly, increased serum UA may have played a key role during human evolution by maintaining the higher blood pressure required to support bipedal motility and upright posture during periods of low salt availability ${ }^{7}$. This is supported by data showing a strong association between hyperuricaemia and hypertension ${ }^{8}$.

Secondly, soluble UA has anti-oxidant properties that could be important for reducing the damage and the risk of developing diseases associated with increased oxidative stress. UA is reported to be as effective as ascorbate in reducing oxidative damage in erythrocytes ${ }^{9}$. This study also suggests that as $\mathrm{UA}$ is present at much higher concentrations than ascorbate in serum it may account for up to $60 \%$ of the anti-oxidant capacity in the serum.

In this way increased serum UA during evolution may have promoted neuroprotection, as the brain is very sensitive to damage induced by oxidative stress. This is supported by recent meta-analyses that further confirm the association between increased serum UA levels and reduced risk and progression of neurological disorders such as Parkinson's disease ${ }^{10}$ and Alzheimer's ${ }^{11}$.

The anti-oxidant properties of UA may also protect humans from multiple sclerosis (MS) by reducing the damage caused by reactive oxygen and nitrogen species ${ }^{12}$. This is corroborated by studies showing lower levels of UA in the serum and cerebrospinal fluid in patients with MS compared to age and sex matched controls ${ }^{13,14}$ and lower UA levels in MS patients with active disease than patients in remission ${ }^{15}$. Despite these clear associations there has been limited success in clinical trials aiming to treat MS by raising serum UA levels ${ }^{16,17}$. 
Thirdly, soluble UA can also have pro-oxidant effects as evidenced by the oxidation of UA by peroxynitrite, which can produce an aminocarbonyl radical $^{18}$, and the reaction of UA with myeloperoxidase that can produce a urate radical $^{19}$. In this way, increased soluble UA may support ROS dependent intracellular signalling as both the NFKB and MAP kinase signalling pathways are redox sensitive $e^{20,21}$. Furthermore, the generation of extracellular ROS by UA oxidation could aid in the killing of pathogens during inflammation. This does not necessarily contradict the anti-oxidant theory described above but indicates that perhaps both the pro- and anti-oxidant effects of increased soluble UA support the evolutionary loss of uricase.

Together these theories and the supporting studies indicate that the loss of uricase and increase in serum UA in early man could have provided a selective advantage and was not just a random occurrence.

\subsection{Dysregulation of UA homeostasis}

\subsubsection{Hypouricaemia (serum UA $<0.12 \mathrm{mM}$ or $<2 \mathrm{mg} / \mathrm{dl}$ )}

Hypouricaemia is rare in humans and can be caused by a heritable loss of function mutation in the urate transporters URAT1 or Glut9 that results in reduced renal reabsorption and excessive urinary excretion of soluble $U^{22-24}$. Alternatively, a loss in function mutation in XOR reduces uric acid production and is the cause of hereditary xanthinuria ${ }^{6}$. The physiological effects of hypouricaemia include urolithiasis and exercise induced acute renal failure ${ }^{25-}$ ${ }^{28}$. This indicates that serum UA may have an important role in maintaining proper kidney function.

\subsubsection{Risk factors for hyperuricaemia (serum UA $>0.42 \mathrm{mM}$ or $>7 \mathrm{mg} / \mathrm{dl}$ )} Age

Serum UA levels increase with age. This is evident from the increased prevalence of hyperuricaemia and gout in the ageing population and may be a result of decreased kidney function and renal UA excretion or a combination of risk factors for hyperuricaemia that all increase with age $\mathrm{e}^{29,30}$. 


\section{Gender}

Men in general have higher serum UA levels than women and are therefore more prone to developing hyperuricaemia but this gender discrepancy decreases after women reach menopause ${ }^{31}$. The UA lowering effect of estrogens have been clearly demonstrated by an inverse relationship between estrogens and serum UA during the menstrual cycle and the association between postmenopausal hormone use and lower serum UA levels ${ }^{32,33}$. Additionally, it has been demonstrated that testosterone treatment can increase serum UA levels ${ }^{34}$ further explaining the clear gender difference present in serum UA levels.

\section{Medication}

A common side effect of many medications is decreased renal function. Most medications require clearance via the kidneys and urinary excretion. As such, medication can have either a general negative effect on renal function or a more specific negative effect on UA excretion. For example, low dose aspirin and diuretics can decrease renal UA excretion by competing for UA with organic ion transporters in the kidney ${ }^{35}$.

\section{Genetics}

UA excretion involves several stages of renal tubular filtration, secretion and reabsorption ${ }^{6}$. URAT1, Glut9, ABCG2 and several other organic anion transporters are responsible for this process and heritable polymorphisms within the genes for these transporters have been identified as major contributors to the risk of developing hyperuricaemia ${ }^{22,36,37}$. This has been further demonstrated in population-based studies linking the prevalence of different genetic polymorphisms to the incidence of gout ${ }^{38-40}$. The rs2231142 polymorphism is a glutamine to lysine mutation at amino acid 141 (Q141K) in the ABCG2 transporter that produces a loss of transporter function which decreases extra-renal UA secretion ${ }^{37,41}$. 
Diet

A purine rich diet, especially one high in seafood, can increase serum UA levels as a result of increased purine metabolism and production of $U A^{42}$. It has been shown that increased alcohol consumption, especially beer, increases the incidence of hyperuricaemia ${ }^{43,44}$. This can be attributed to the high purine content of beer and the convergence of the purine and ethanol metabolic pathways. Ethanol metabolism increases degradation of ATP, which enhances purine metabolism and results in increased UA production ${ }^{45}$. Ethanol metabolism also increases lactate production, which is a competitive inhibitor of UA excretion ${ }^{46,47}$. Excess fructose consumption, especially from sugar-sweetened beverages, has also been shown to contribute to hyperuricaemia ${ }^{48,49}$. The mechanism is similar to ethanol, in that fructose metabolism increases purine nucleotide metabolism and lactate production ${ }^{50}$ and may also involve a direct effect of increased glucose and fructose on urate transport, as these sugars are also substrates for the Glut9 transporter ${ }^{51}$.

\section{Lifestyle}

Healthy eating and exercise habits are also important factors in maintaining normal serum UA levels. This has been demonstrated with interventional trials where treatment of patients with gout or obesity consisted of caloric restriction $^{52}$ or a low calorie diet and exercise ${ }^{53}$. It was found that these treatments lowered serum UA levels and improved renal UA excretion indicating that a poor diet and sedentary lifestyle can contribute to hyperuricaemia.

Ultimately, these risk factors combine together to increase production and/or decrease excretion of soluble UA, increasing the risk of developing hyperuricaemia. 


\subsection{Diseases associated with UA dysregulation}

While humans may have evolved to have higher UA levels compared to other mammals this has predisposed humans to developing hyperuricaemia when control of UA homeostasis is perturbed. Hyperuricaemia is associated with a plethora of diseases but the role of soluble UA in exacerbating the pathophysiology of these diseases is still not fully understood.

\subsubsection{Kidney disease}

Hyperuricaemia is recognised as a risk factor for the development of acute kidney injury. This is clearly demonstrated during tumour lysis syndrome where massive tumour cell death occurs as a result of chemotherapy for haematological malignancy ${ }^{54}$. The breakdown and release of tumour cell contents into the blood stream causes serum UA levels to spike and can result in severe renal nephropathy ${ }^{55}$. However, in chronic kidney disease it is difficult to separate the independent effects of raising serum UA levels from other pathologies associated with hyperuricaemia and kidney disease. As such, definitive causation has not yet been shown in humans ${ }^{6}$.

\subsubsection{Hypertension and cardiovascular disease}

Hypertension is closely associated with hyperuricaemia but whether the high serum UA levels cause hypertension is still the subject of debate. Uratelowering therapy (ULT) has been shown to reduce essential hypertension ${ }^{56}$. However, the association between hypertension and hyperuricaemia weakens with increasing patient age and duration of hypertension ${ }^{57}$. Additionally, there has been a lack in consistency between genome wide association studies investigating the effects of polymorphisms in UA transporters on hypertension and Johnson et al. posit that this could stem from a difference in function between intracellular and serum $U A^{58}$. In this case, hyperuricaemia without hypertension could result from more active urate transporters reducing intracellular UA levels to decrease endothelial dysfunction and vascular stiffening but the increased urate transporter function would also increase serum UA levels. 
Hyperuricaemia is only weakly associated with heart disease and this link can potentially be explained by the association of hyperuricaemia with hypertension, a true risk factor for heart disease. However, hyperuricaemia has been shown to induce endothelial dysfunction by reducing eNOS activity in endothelial cells ${ }^{59-61}$ and endothelial dysfunction, a predictor of cardiovascular disease, precedes atherosclerotic plaque formation ${ }^{3,62}$. Therefore, it could be argued that hyperuricaemia is indeed a risk factor for cardiovascular disease progression through its contribution to endothelial dysfunction. This link is supported by a study where hyperuricaemia was an independent predictor of mortality in patients with type 2 diabetes and coronary artery disease ${ }^{63}$.

\subsubsection{Type 2 diabetes and obesity}

These diseases share common characteristics such as dyslipidaemia, insulin resistance and low-grade chronic inflammation. However, the contribution of hyperuricaemia to the pathogenesis of these disorders is still unclear.

Hyperuricaemia is associated with an increased risk of developing type 2 diabetes and there is an argument for hyperuricaemia, as a result of excessive fructose intake, being a significant contributor to the current metabolic syndrome and diabetes epidemic ${ }^{64,65}$. Like cardiovascular disease, the contribution of hyperuricaemia to type 2 diabetes may be through its contribution to endothelial dysfunction and low-grade chronic inflammation, as demonstrated in a recent study connecting new onset diabetes in hypertensive patients to hyperuricaemia ${ }^{66}$. Another study showed a direct effect of soluble UA in producing intracellular oxidative damage that inhibited the growth of pancreatic $\beta$-cells in vitro ${ }^{67}$. However, an earlier study found that hyperuricaemia increased the risk of developing hypertension but not diabetes, indicating hyperuricaemia may not be a suitable prognostic marker for diabetes in every situation ${ }^{68}$. 
Obesity can be classified into either metabolically healthy or unhealthy, which pertains to a decreased or increased risk of developing cardiovascular disease respectively ${ }^{69}$. Interestingly, one of the main predictors of unhealthy obesity is hyperuricaemia but obesity itself can contribute to hyperuricaemia by increasing purine metabolism within adipose tissue ${ }^{70}$. This highlights the difficulty of differentiating between the contribution of hyperuricaemia to the disease and the contribution of the disease to hyperuricaemia.

Experimentally, soluble UA activates MAP kinase signalling in adipocytes through increased ROS production and oxidative stress ${ }^{71}$. Another study showed that adipocytes treated with soluble UA decreased production of the anti-inflammatory adipokine adiponectin and increased production of CCL2, a major monocyte chemokine, and that lowering serum UA in obese mice reduced insulin resistance and adipose tissue macrophages ${ }^{72}$. These results indicate that hyperuricaemia directly contributes to metabolic syndrome related pathology by increasing oxidative stress.

Together these studies indicate that elevated serum UA is an indicator of general metabolic dysfunction that may also contribute to the pathology of metabolic syndrome related disorders. However, recent epidemiological studies using Mendelian randomisation, which uses traits linked to genetic polymorphisms of known susceptibility to control for observational bias and confounding co-morbidities, suggest that there is no causal effect of hyperuricaemia in metabolic syndrome related disorders and may be confounded by a causal effect of increased body mass index on serum UA levels and risk of developing hyperuricaemia ${ }^{73-75}$.

\subsubsection{Gout}

The one metabolic disorder that has been definitively linked to hyperuricaemia is gout. Gout is caused by the formation of monosodium urate (MSU) crystals in joints and periarticular spaces ${ }^{76}$. These crystals trigger a severe, selflimiting auto-inflammatory response ${ }^{77}$. At physiological temperature in biological fluids UA has an upper solubility limit of $0.42 \mathrm{mM}(7 \mathrm{mg} / \mathrm{dl})$ and 
above this level the risk of an acute gouty attack increases as the hyperuricaemia worsens ${ }^{78}$.

As the crystallisation event is key to the pathophysiology of gout it is clear that hyperuricaemia is only a risk factor for gout while crystallisation of UA and the ensuing auto-inflammation is the cause. This is an important distinction, as it defines the immunological action of crystalline UA as functionally different from soluble UA (discussed further in section1.8).

\subsection{Treatment of hyperuricaemia}

Currently, treatment for hyperuricaemia is limited to two main indications. The first, and most common, is gout. The second is tumour lysis syndrome.

There are three mechanisms through which UA levels can be pharmacologically manipulated (figure 1.2) and by combining these with diet and lifestyle changes hyperuricaemia can be effectively treated and controlled in the majority of individuals. 


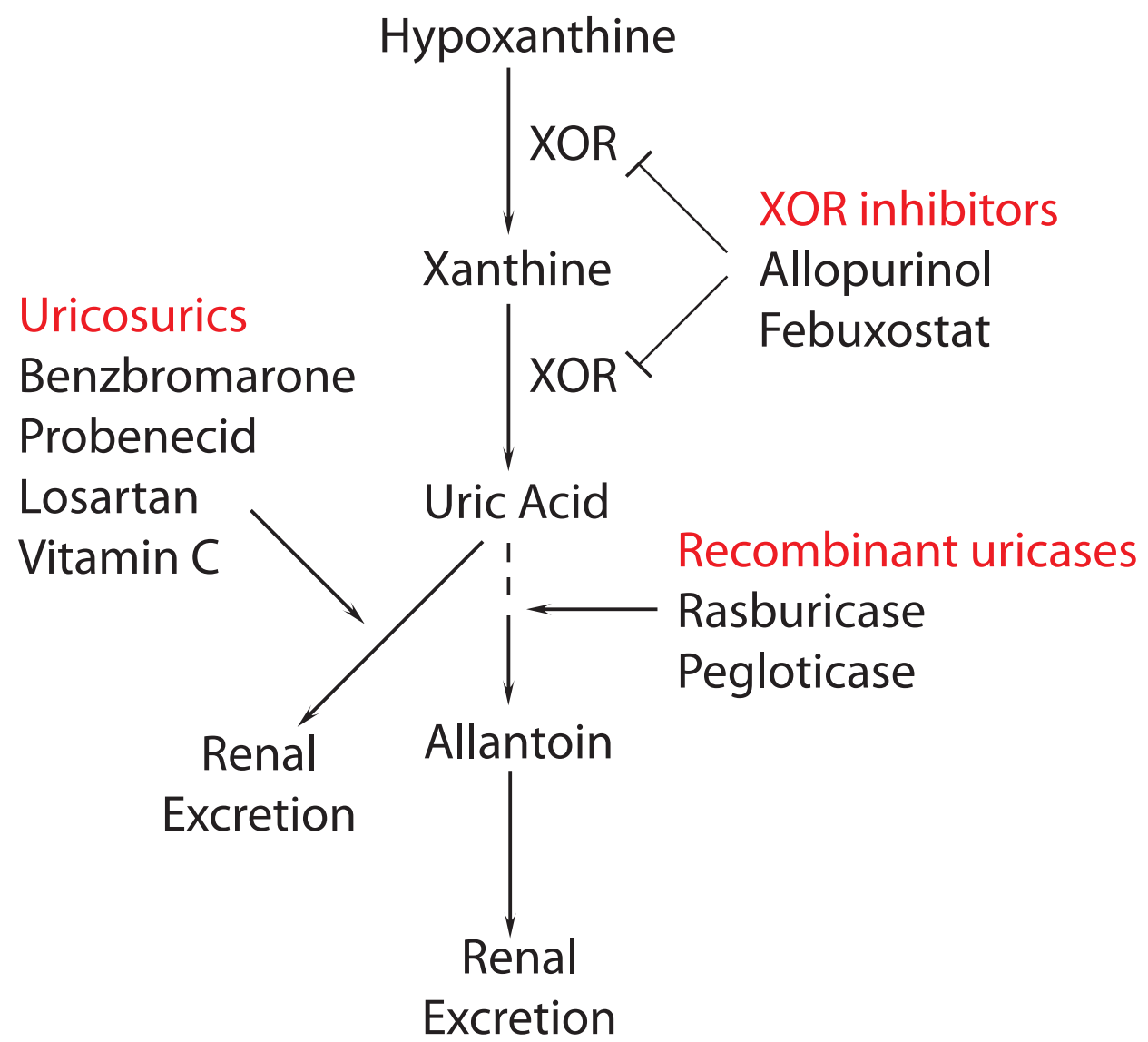

Figure 1.2 Different mechanisms of pharmacological urate-lowering therapy. $X O R$ inhibitors prevent formation of UA, recombinant uricase therapy metabolises excess $U A$ and uricosurics increase renal $U A$ excretion. $X O R=$ xanthine oxidoreductase.

\subsubsection{Xanthine oxidoreductase (XOR) inhibition}

$\mathrm{XOR}$ is the enzyme that produces UA from hypoxanthine and xanthine (figure 1.2) making $X O R$ a logical target for pharmacological inhibition. However, the efficacy of XOR inhibition hinges on efficient renal UA clearance. For example, people with genetic variants in urate transporters may not respond well to XOR inhibition because efficient renal UA reabsorption reduces the clearance rate of excess UA.

From an immunological standpoint $\mathrm{XOR}$ is recognised as a significant source of reactive oxygen species (ROS), which are involved in inflammatory signalling and vascular oxidative stress ${ }^{79,80}$. Therefore, XOR inhibition will reduce both UA and ROS. Being able to differentiate between the effects of 
lowering serum UA and decreasing ROS is an important consideration when assessing the immunological outcomes of UA reduction with a XOR inhibitor.

\section{Allopurinol}

Allopurinol has been the mainstay of urate-lowering therapy (ULT) for the past 40 years as it is generally well tolerated and has few serious side effects ${ }^{81}$. In certain cases treatment with allopurinol can result in the precipitation of an acute gout attack, the aetiology of which is still unknown. Less likely, but more severe, is development of allopurinol hypersensitivity syndrome (AHS). The presence of the HLA-B*5801 allele has been linked with an increased risk of developing AHS but the exact mechanism is unknown ${ }^{82}$. These side effects have resulted in the development of cautious dosing regimes that start at 50 or $100 \mathrm{mg} /$ day and escalate slowly until target serum UA is achieved at which point the allopurinol dose is maintained ${ }^{83}$.

\section{Febuxostat}

Febuxostat is the only other XOR inhibitor to come onto the market and has only done so in the last few years. Comparisons with allopurinol have shown febuxostat to be more selective in its inhibition of $X \mathrm{R}^{84}$ and to provide better UA lowering with a lower dose ${ }^{85-87}$. Febuxostat has a small human side effect profile, however there have been reports of neutropenia or cardiovascular thromboembolic events as a result of febuxostat treatment ${ }^{88,89}$.

\subsubsection{Recombinant uricase}

The development of recombinant uricase for the treatment of hyperuricaemia is relatively recent. Uricase breaks down UA into HIU (figure 1.1), which in the absence of HIU-hydrolase and OHCU-decarboxylase in humans can spontaneously degrade into allantoin but at a much slower rate than when enzymatically degraded ${ }^{4}$. Currently, the immunological and physiological functions of HIU and $\mathrm{OHCU}$ production during treatment of hyperuricaemia with recombinant uricase are unknown but need to be investigated to 
determine the safety and suitability of recombinant uricase treatment. Recently, a mouse strain has been developed that has a loss of function mutation in the Urah gene that codes for HIU hydrolase and has been designated Urah ${ }^{\text {Plt2/Plt2 }}\left(\mathrm{PLT}_{2}\right)^{90}$. The PLT2 strain has some growth abnormalities including hepatomegaly, increased platelet counts and development of spontaneous liver cancer after two years. However the role of increased HIU in these pathologies was not investigated.

There are two recombinant uricase formulations currently approved for use in treating hyperuricaemia. Rasburicase is derived from Aspergillus flavus and pegloticase is of porcine origin and has been conjugated to polyethylene glycol $^{91}$. Both rasburicase and pegloticase have been successful in the treatment of tumour lysis syndrome and can be prescribed for use in treatment-failure gout, where the patient has had an adverse reaction to allopurinol or allopurinol treatment has been ineffective ${ }^{92}$. However, there are safety concerns over the antigenicity of recombinant uricases that currently preclude them for long-term repeated use until studies on the effects of longterm treatment are complete ${ }^{93}$.

\subsubsection{Uricosurics}

The third method of ULT is through uricosuria. This works by increasing renal clearance of UA and are recommended for treating hyperuricaemia in cases of renal UA underexcretion ${ }^{94}$. Three uricosurics are used clinically: benzbromarone, probenecid and losartan. These inhibit the URAT1 transporter to prevent UA reabsorption in the proximal kidney tubule ${ }^{22,95}$. Benzbromarone was initially well received but has since been withdrawn from many countries due to the increased risk of hepatotoxicity ${ }^{94,96}$. Probenecid is the most widely used uricosuric and can be used as a monotherapy for allopurinol refractory gout and also in combination with allopurinol ${ }^{97}$. Losartan is prescribed primarily for treatment of hypertension but has off label uricosuric effects that make it a promising candidate for combined treatment of hypertension and hyperuricaemia, two conditions with a high rate of comorbidity ${ }^{98,99}$. Interestingly, a recent meta-analysis found that vitamin $\mathrm{C}$ 
supplementation reduced serum UA levels in randomised controlled trials and implicates competitive inhibition of UA reabsorption through renal transporters as a possible mechanism ${ }^{100}$. Given the reported safety of high dose vitamin $\mathrm{C}^{101}$ this may provide an avenue for use in combination therapy of hyperuricaemia.

\subsubsection{Diet and lifestyle}

The association between excessive food consumption and gout is best exemplified by the moniker "disease of kings" which is how gout was perceived right up until the $20^{\text {th }}$ century and long before hyperuricaemia was recognised as the primary risk factor ${ }^{102}$. However, there has been a rapid increase in the late $20^{\text {th }}$ century in the prevalence of hyperuricaemia and associated metabolic diseases that coincides with the increased availability of calorie rich foods and increased sedentary lifestyle in western and westernising countries ${ }^{103,104}$. Education on how diet and lifestyle changes can be used to help ameliorate hyperuricaemia is common practice when initiating ULT for gout. Simple measures such as lowering consumption of alcoholic and fructose sweetened beverages or getting regular exercise can have a significant effect on the reduction of gout flares and metabolic comorbidities associated with hyperuricaemia ${ }^{83,105}$. However, diet and lifestyle modifications alone can sometimes be insufficient to significantly reduce serum UA levels and are more effective when combined with the right pharmacotherapy ${ }^{106}$.

\subsection{Immunological effects of UA}

\subsubsection{Crystalline monosodium urate (MSU)}

The crystalline form of uric acid, MSU, is more than just the etiological agent in gout. UA was first identified as a powerful endogenous immune adjuvant in an elegant study by Shi et al. showing that UA was released from dying cells and was able to enhance adaptive immune responses by licensing DC in the presence of antigen ${ }^{107}$ (figure 1.3). They also demonstrated that by increasing and decreasing UA in vivo they could potentiate or restrict the generation of 
CD8 T-cell responses respectively. These results led them to hypothesise that MSU was responsible for this adjuvanticity. To complement this research, the adjuvanticity of MSU has been recently demonstrated in a model of cancer immunotherapy whereby co-injection of $\mathrm{MSU}$ and $M$. smegmatis slowed tumour progression through the rapid induction of monocyte-derived $D^{108}$.

The mechanism through which MSU activates immune cells is not fully understood. Knockout of CD14 on monocyte/macrophages reduces the inflammatory response to $\mathrm{MSU}^{109}$. Antibody blocking experiments have shown that the $F \mathrm{c} \gamma$ receptor $\mathrm{CD} 16$ and adhesion molecule $\mathrm{CD} 11 \mathrm{~b}$ mediate activation of neutrophils to $\mathrm{MSU}^{110}$. These studies do not definitively show that receptor ligation of MSU by these molecules triggers the down stream inflammatory effects. However, they do indicate that the involvement of these receptors enhances inflammatory signalling in the case of CD14 or phagocytosis of MSU crystals in the case of CD16 and CD11b. This is supported by research showing that lipid sorting occurs upon contact of MSU crystals with the cellular membrane and activates the Syk kinase dependent signalling pathway which can initiate phagocytosis and inflammatory cytokine production $^{111,112}$.

The NLRP3 inflammasome is a multi-protein complex involved in the cleavage of pro-IL-1 $\beta$ and pro-IL-18 into their active forms ${ }^{113}$. The key components are NLRP3, the adaptor protein ASC and pro-caspase1. Activation of the NLRP3 inflammasome brings these components together to trigger pro-caspase 1 by autocatalytic cleavage. Active caspase 1 can then cleave pro-IL-1 $\beta$ into active IL-1 $\beta$ for secretion. 


\section{Tissue damage or infection}

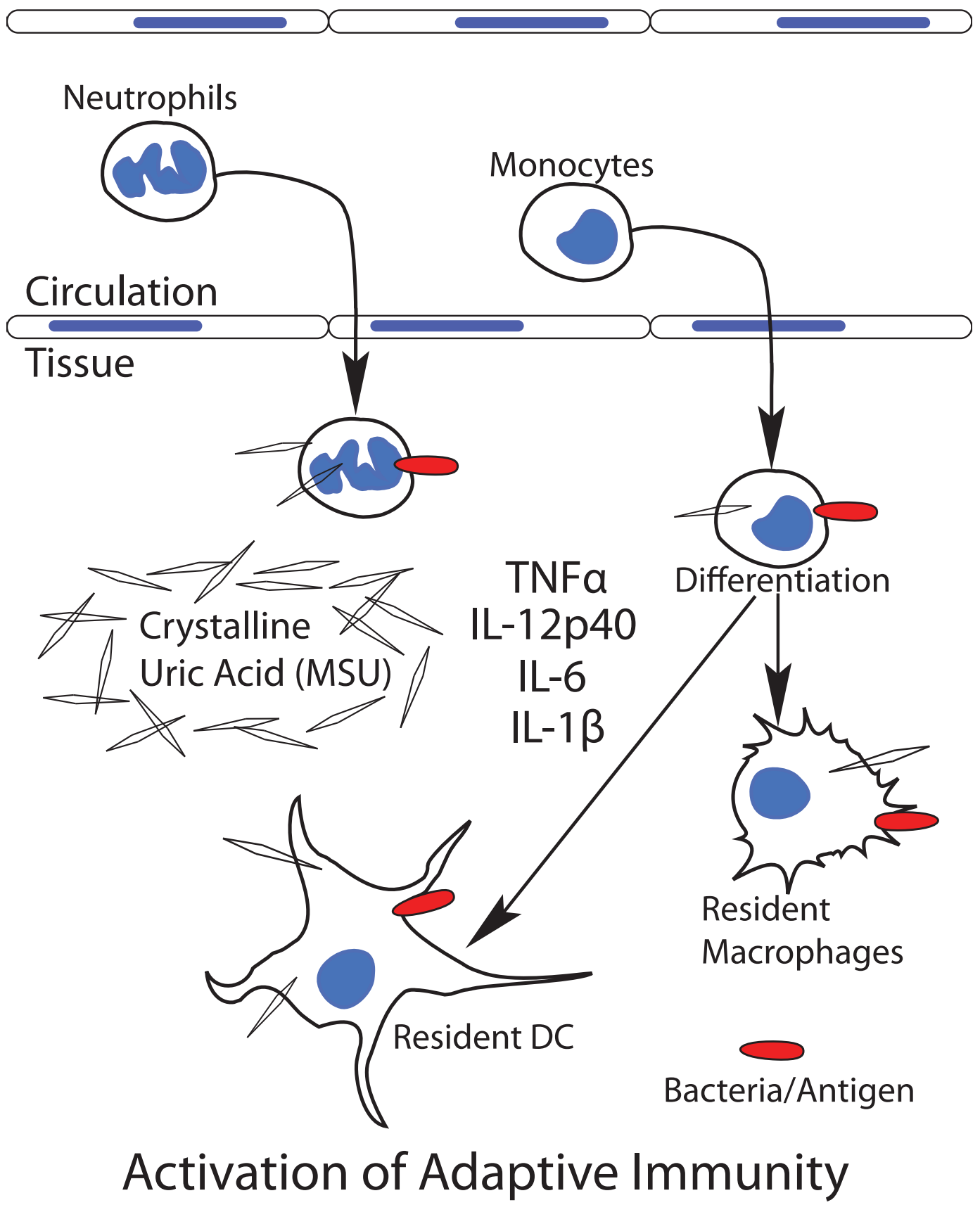

Figure 1.3 Adjuvanticity of MSU.

UA released from dying cells can form MSU crystals to activate the innate immune system (neutrophils and monocytes) and promote differentiation of monocytes into inflammatory $D C$, in the presence of antigen, to activate an adaptive immune response. 
Endogenous danger signals such as ATP, CPPD and MSU can activate the NLRP3 inflammasome. However, it wasn't until the recognition of MSU as an endogenous danger signal that Martinon and colleagues investigated the role MSU played in IL-1 $\beta$ maturation. It was discovered that MSU is a potent activator of the NLRP3 inflammasome and IL- $1 \beta$ maturation ${ }^{114}$. This discovery has led to a renewed interest in inflammasomes and the identification of many different inflammasomes in both mice and humans ${ }^{115}$. However, the NLRP3 inflammasome is by far the best characterised.

\subsubsection{Soluble UA}

The role of soluble UA in inflammation and immunity has not been as extensively investigated. In contrast to MSU there are reports that soluble UA has an anti-inflammatory effect on inflammation. UA administration has been shown to reduce the severity of dsRNA-induced arthritis in mice, possibly through a decrease in granulocyte inflammatory activity ${ }^{116}$. Additionally, UVinduced skin damage was shown to increase local soluble UA levels and immune suppression in vivo that could be both be inhibited with allopurinol treatment ${ }^{117}$. However, an inflammatory role for soluble UA has been proposed in obesity and metabolic syndrome whereby increased soluble UA levels increased inflammatory signalling in adipocytes and contributed to insulin resistance ${ }^{71,72}$. These effects were mediated by the pro-oxidant capabilities of soluble UA and demonstrate that soluble UA may have different functions depending on the specific cell type.

\subsection{Monocytes and metabolic disease}

Monocytes play multiple roles in innate immunity and in the crosstalk between innate and adaptive immunity. They are both effector and precursor cells that can alter their function as they adapt to the local environment and immunological stimuli. Monocytes produced in the bone marrow are recruited into the circulation by the chemokine-chemokine receptor axis CCL2-CCR2 ${ }^{118}$. In the circulation monocytes make up approximately $5 \%$ of all immune cells and can be split into two contiguous sub-populations. The CD14+/CD16- 
subset (Ly6C+ in mice) are circulating monocytes that are recruited to sites of inflammation, whereas CD14+/CD16+ monocytes (Ly6C- in mice) patrol in and out of the tissues and may be the population that replenishes the tissue resident macrophage pool ${ }^{119,120}$. There is also a large population of monocytes resident in the spleen that can be recruited into the circulation and then to the tissues during an inflammatory event ${ }^{121}$.

In the tissues monocytes initially respond to inflammatory stimuli by secreting cytokines and chemokines that activate and educate the immune system to combat the insult and begin the resolution process. They also begin differentiating into macrophages or dendritic cells (DC) depending on the nature of the inflammation. Macrophage differentiation occurs in sterile inflammation such as gout ${ }^{122}$. Monocyte-derived DC can be produced in response to adjuvant therapy and delay the growth of established tumours ${ }^{108}$. As they differentiate monocytes gain phagocytic capacity and the phenotypic and functional characteristics that the local environment requires for an effective innate or adaptive immune response to the specific insult.

Despite their essential role during pathogen-induced inflammation, or perhaps because of it, monocytes are also key immune cells involved in the pathogenesis of metabolic disease.

\subsubsection{Kidney disease}

Cisplaten-induced acute renal failure is used as a model of tumour lysis syndrome-mediated renal injury and induces monocyte recruitment into the kidney. In this model hyperuricaemia exacerbated renal tubular injury compared to rasburicase treated rats, indicating a role for increased serum UA in exacerbating renal inflammation through increased monocyte recruitment ${ }^{123}$. Additionally, increased renal vasculopathy and macrophage infiltration was observed in hyperuricaemic mice after remnant kidney surgery $^{124}$. However, blood monocytes isolated from patients with end stage renal disease were less inflammatory when stimulated ex vivo with LPS or MSU, indicating that the monocytes that invade as a result of renal 
nephropathy are immunosuppressed and as such may be mitigating further disease $^{125}$.

\subsubsection{Hypertension and cardiovascular disease}

Hypertension is associated with the recruitment of monocyte/macrophages into renal tissue. Here the macrophages can contribute to renal dysfunction and the development of hypertension through a combination of increased oxidative stress and angiotensin II activity ${ }^{126}$.

During the formation of atherosclerotic plaques monocytes are recruited to the inflamed arterial wall and invade. Once inside the plaque, monocytes differentiate into macrophages and phagocytose the cholesterol crystals in an attempt to repair the damaged artery wall. Excessive cholesterol uptake can create a chronic cycle of macrophage foam cell formation, cell death and more monocyte recruitment ${ }^{127}$. In addition to this, hyperuricaemia is associated with increased monocyte recruitment into the circulation and soluble UA can stimulate CCL2 production, a major monocyte chemokine, in vascular smooth muscle cells ${ }^{128,129}$. This could potentially increase monocyte recruitment to atherosclerotic plaques in hyperuricaemic individuals.

\subsubsection{Type 2 diabetes and obesity}

Adipokines are the signalling molecules that control energy usage and fat deposition and monocytes express adipokine receptors, such as the leptin receptor, allowing them to respond to changes in metabolic signalling ${ }^{130}$. Their role as instigators or moderators of inflammation is still unknown, however monocytes respond to leptin by increasing their phagocytic capacity and cytokine production ${ }^{131}$. Furthermore, in patients with type 2 diabetes monocytes display an enhanced inflammatory phenotype and are more reactive when stimulated ex vivo ${ }^{132,133}$ and adipose tissue macrophages are recognised as a driver of obesity related inflammation and insulin resistance $^{134}$. This indicates that monocyte recruitment into adipose tissue could be an important factor in determining the severity and progression of disease and there may be an opportunity to target monocyte recruitment in 
the treatment of metabolic syndrome. However, whether hyperuricaemia has a direct impact on monocyte recruitment to adipose tissue during type 2 diabetes and obesity is unclear.

\subsubsection{Gout}

The role of monocytes and macrophages in gout has been well studied. Monocytes are recruited in large numbers into the inflamed synovium following initiation of an acute gouty attack $^{77}$. Interestingly, resident macrophages are not required for this monocyte recruitment as the synovial epithelial cells are the main source of monocyte chemoattractants ${ }^{135}$. The monocytes that are recruited into an inflamed joint during gout are initially refractory to further MSU stimulation but gradually differentiate into hyper responsive resident macrophages that when stimulated produce an even more severe auto-inflammatory gouty attack $^{136}$.

\subsection{Experimental hyperuricaemia}

In an effort to better understand the physiological and immunological effects of hyperuricaemia there have been many animal models developed.

The majority of studies have used rodents as model animals, employing pharmacological, genetic and dietary manipulation of purine metabolism. Other organisms have also been investigated, such as silkworms for their use in testing new $\mathrm{ULT}^{137}$ and Dalmatians, as they have a unique heritable decrease in UA reabsorption that may be the result of endocrine signalling from the liver ${ }^{138}$.

\subsubsection{Pharmacological manipulation}

The most common pharmacological method of inducing hyperuricaemia is through the inhibition of uricase with oxonic acid, which is often delivered by intraperitoneal (i.p.) injection or orally and can be used to induce both acute and chronic hyperuricaemia. Many studies using oxonic acid are designed to test the efficacy of new compounds or extracts to lower serum UA levels ${ }^{139-141}$ 
but oxonic acid is also used for research into the effects of hyperuricaemia on metabolic dysfunction ${ }^{142,143}$.

Inosine, a purine precursor that is broken down into UA has been used to raise serum UA levels in mice and was successful in treating experimental autoimmune encephalomyelitis ${ }^{144}$.

\subsubsection{Genetic manipulation}

Genetic ablation of the uricase enzyme has been used to create a mouse model of hyperuricaemia ${ }^{145}$. However, this mouse strain develops severe hyperuricaemia and nephropathy, as a result of MSU crystal deposition in the kidneys, followed quickly by death. Therefore, its use as an experimental mouse strain is limited, as it requires constant ULT for long-term survival.

A hyperuricaemic mouse deficient in the UA transporter Glut9 has been developed $^{146}$. Glut9 is important for UA transport into the liver, the main location of uricase, so this knockout develops hyperuricaemia as a result of impaired UA metabolism rather than underexcretion.

Indirectly, models of hyperuricaemia have been created during development of genetically obese mice. This is exemplified by the leptin deficient (ob/ob) and leptin receptor deficient ( $d b / d b$ and Pound) mouse strains, which all develop hyperuricaemia alongside obesity and symptoms of metabolic dysfunction. The effect of lowering serum UA with allopurinol on renal disease has been investigated in $d b / d b$ mice and was shown to reduce inflammatory cell infiltration via a reduction in renal epithelial adhesion molecule expression, specifically ICAM $1^{147}$. Allopurinol treatment has also been shown to alleviate insulin resistance and hepatic steatosis in Pound mice by reducing oxidative stress $^{72,148}$. The ob/ob mouse strain has been used to determine the effect of obesity on UA transporter expression and found that both ob/ob and high fat diet fed mice both increased renal expression of URAT1 and ABCG $2^{149}$. 


\subsubsection{Dietary manipulation}

A diet consisting of a purine rich yeast extract has been used to model hyperuricaemia. This model tested the ability of potential therapeutic compounds to lower serum uric acid levels ${ }^{150}$.

Hyperuricaemia develops alongside obesity and metabolic dysfunction in high fat diet models. These models have been used are to test the efficacy of cysteine-containing compounds ${ }^{151}$ and polyphenol-enriched extracts ${ }^{152}$ to reduce metabolic dysfunction .

A high fructose diet has also been shown to induce hyperuricaemia and metabolic dysfunction in rats where the metabolic dysfunction was attributed to the increased serum UA promoting endothelial dysfunction ${ }^{153}$. Metabolic dysfunction has been linked to hyperuricaemia in fructose fed rats whereby the XOR inhibitor febuxostat has reduced hyperuricaemia, metabolic dysfunction and renal disease when used therapeutically ${ }^{154}$. Additionally, another study has shown that arginase inhibition alleviated hypertension in fructose fed rats by re-establishing the balance of $\mathrm{NO}$ and ROS production but had no impact on hyperuricaemia ${ }^{155}$. This indicates that hyperuricaemia may contribute to hypertension and other metabolic disturbances through increased arginase activity.

\subsection{Aims}

This research aims to develop a better understanding of the role monocytes play in hyperuricaemia associated diseases with the ultimate goal of determining the potential therapeutic benefits of modulating serum UA to help combat human diseases associated with metabolic dysfunction.

1: To investigate the direct effect of in vitro soluble UA on the inflammatory response of human blood monocytes.

2: To define the in vivo effects of raising and lowering serum UA on systemic and monocyte responses to LPS. 
Chapter 2:

Materials and Methods 



\section{Materials and Methods}

\subsection{Materials}

\subsubsection{Reagents and kits}

\begin{tabular}{|c|c|}
\hline Reagent/kit & Company \\
\hline Uric acid detection kit & Abcam (Cambidge, UK) \\
\hline UASure test strips & ApexBio (Taiwan) \\
\hline Limulus amebocyte aysate kit & $\begin{array}{c}\text { Associates of Cape Cod (East } \\
\text { Falmouth, MA) }\end{array}$ \\
\hline Polymorphprep density gradient & Axis-Shield (Oslo, Norway) \\
\hline $\begin{array}{l}\text { ELISA: human IL-12p40, mouse IL-10, } \\
\text { IL-12p40, IL-6, TNF } \alpha\end{array}$ & BD (Auckland, NZ) \\
\hline GolgiStop & BD Biosciences (Auckland, NZ) \\
\hline Propidium iodide & BD Biosciences (Auckland, NZ) \\
\hline Dc protein assay reagent & Bio-Rad (Auckland, NZ) \\
\hline Lympholyte M density gradient & Cedarlane (Burlington, Canada) \\
\hline $\begin{array}{l}\text { 1-Methoxy-5-methylphenazinium } \\
\text { methylsulfate (mPMS) }\end{array}$ & Dojindo (Kumamoto, Japan) \\
\hline $\begin{array}{l}\text { 2-(4-Iodophenyl)-3-(4-nitrophenyl)-5- } \\
\text { (2,4-disulfophenyl)-2H-tetrazolium } \\
\text { (WST-1) }\end{array}$ & Dojindo (Kumamoto, Japan) \\
\hline Bovine serum albumin (BSA) & Life Technologies (Auckland, NZ) \\
\hline Dihidrorhodamine-123 (DHR-123) & Life Technologies (Auckland, NZ) \\
\hline $\begin{array}{l}\text { Dynabeads untouched human } \\
\text { monocytes }\end{array}$ & Life Technologies (Auckland, NZ) \\
\hline Ethylenediaminetetraacetic acid (EDTA) & Life Technologies (Auckland, NZ) \\
\hline $\begin{array}{l}\text { Luminex multiplex cytokine array: } \\
\text { human IL-10, IL12p40, IL-1 } \beta \text {, IL-6, } \\
\text { TNF } \alpha \text {, IL-1Ra, IL-8, CCL2, CCL3, } \\
\text { CCL4, CCL5, CCL7. }\end{array}$ & Life Technologies (Auckland, NZ) \\
\hline
\end{tabular}




\begin{tabular}{|l|c|}
\hline ELISA: human IL-1 $\beta$ & $\begin{array}{c}\text { Mabtech (Thomastown, } \\
\text { Australia) }\end{array}$ \\
\hline Streptavidin-MACS beads & $\begin{array}{c}\text { Miltenyi Biotec (Cologne, } \\
\text { Germany) }\end{array}$ \\
\hline $\begin{array}{l}\text { 4-12\% Bis-Tris mini gels } \\
\text { IL-1 } \beta\end{array}$ & $\begin{array}{c}\text { Life Technologies (Auckland, NZ) } \\
\text { R }\end{array}$ \\
\hline Adenosine
\end{tabular}


2.1.2 Antibodies

\begin{tabular}{|c|c|c|}
\hline Antibody & Conjugate & Company \\
\hline DUSP10 & - & Abcam (Cambidge, UK) \\
\hline NFкB p50 & - & Abcam (Cambidge, UK) \\
\hline NLRP3 & - & Abcam (Cambidge, UK) \\
\hline CD14 & $\mathrm{PE}$ & BD (Auckland, NZ) \\
\hline ICAM1 & $\mathrm{PE}$ & BD (Auckland, NZ) \\
\hline Annexin V & FITC & BD Pharmingen (Auckland, NZ) \\
\hline CD11b & APC-Су7 & BD Pharmingen (Auckland, NZ) \\
\hline IL-10 & APC & BD Pharmingen (Auckland, NZ) \\
\hline CD16 & PE-Cy7 & Biolegend (San Diego, CA) \\
\hline CD62L & FITC & Biolegend (San Diego, CA) \\
\hline $\mathrm{I \kappa B} \alpha$ & - & Biolegend (San Diego, CA) \\
\hline Ly6C & PerCP-Сy5.5 & Biolegend (San Diego, CA) \\
\hline NFкB p50 & - & Biolegend (San Diego, CA) \\
\hline TLR4 & $\mathrm{PE}$ & Biolegend (San Diego, CA) \\
\hline NFкB p65 & - & Biolenged (San Diego, CA) \\
\hline $\mathrm{IL}-1 \beta$ & - & Biovision (Milpitas, CA) \\
\hline p-DUSP1 & - & Cell Signaling (Danvers, MA) \\
\hline p-ERK1/2 & - & Cell Signaling (Danvers, MA) \\
\hline p-p38 & - & Cell Signaling (Danvers, MA) \\
\hline poly-lgG & - & CSL Bioplasma (Melbourne, Australia) \\
\hline B220 & Biotin & eBioscience (San Diego, CA) \\
\hline CD11b & APC & eBioscience (San Diego, CA) \\
\hline CD11b & $\mathrm{PE}$ & eBioscience (San Diego, CA) \\
\hline CD14 & APC & eBioscience (San Diego, CA) \\
\hline CD162 & APC & eBioscience (San Diego, CA) \\
\hline CD3 & Biotin & eBioscience (San Diego, CA) \\
\hline CD31 & PE-Cy7 & eBioscience (San Diego, CA) \\
\hline $\mathrm{Bcl} 3$ & - & GeneTex (Irvine, CA) \\
\hline TBP & - & GeneTex (Irvine, CA) \\
\hline $2.4 \mathrm{G} 2$ & - & In-house \\
\hline
\end{tabular}




\begin{tabular}{|l|c|c|}
\hline Caspase1 & - & Life Technologies (Auckland, NZ) \\
\hline ASC & - & Novus Biologicals (Littleton, CO) \\
\hline DUSP1 & - & Santa Cruz Biotechnology (Dallas, TX) \\
\hline Mouse $2^{\circ}$ & HRP & Santa Cruz Biotechnology (Dallas, TX) \\
\hline Rabbit 2 & HRP & Santa Cruz Biotechnology (Dallas, TX) \\
\hline$\alpha$-tubulin & - & Sigma-Aldrich (Auckland, NZ) \\
\hline$\beta$-actin & - & Sigma-Aldrich (Auckland, NZ) \\
\hline
\end{tabular}

\subsubsection{Commercial buffers and media}

\begin{tabular}{|l|r|}
\hline Buffer/Media & Company \\
\hline $\begin{array}{l}\text { Hank's balanced salt solution, } \\
\mathrm{Ca}^{2+} \text { and } \mathrm{Mg}^{2+} \text { free (HBSS) }\end{array}$ & Life Technologies (Auckland, NZ) \\
\hline RPMI-1640 & Life Technologies (Auckland, NZ) \\
\hline Penicillin-Streptomycin & Life Technologies (Auckland, NZ) \\
\hline Glutamax & Life Technologies (Auckland, NZ) \\
\hline $\begin{array}{l}\text { Dulbecco's phosphate } \\
\text { buffered saline (DPBS) }\end{array}$ & Life Technologies (Auckland, NZ) \\
\hline 4x sample loading buffer & Life Technologies (Auckland, NZ) \\
\hline $10 x$ sample reducing buffer & Life Technologies (Auckland, NZ) \\
\hline MES running buffer & Life Technologies (Auckland, NZ) \\
\hline RBC lysis buffer & Qiagen (Germantown, MD) \\
\hline
\end{tabular}

\subsubsection{In-house buffers and media}

Salts and buffer constituents were sourced from Sigma-Aldrich (Auckland, NZ) unless stated otherwise in sections 2.1.1 or 2.1.3.

Autologous human serum (ahs)RPMI

RPMl-1640 with 100 units $/ \mathrm{ml}$ penicillin, $100 \mu \mathrm{g} / \mathrm{ml}$ streptomycin, 1x glutamax and $5 \%$ heat inactivated autologous human serum. 
Serum free (sf)RPMI

RPMI-1640 with 100 units $/ \mathrm{ml}$ penicillin, $100 \mu \mathrm{g} / \mathrm{ml}$ streptomycin, $1 \mathrm{x}$ glutamax.

FACS buffer

DPBS with $0.1 \%$ BSA and $0.01 \%$ sodium azide.

Saponin buffer

DPBS with $0.1 \%$ saponin, $0.1 \%$ FBS and $0.1 \%$ sodium azide.

Annexin $V$ binding buffer

Distilled water with $10 \mathrm{mM}$ HEPES, $140 \mathrm{mM} \mathrm{NaCl}$ and $2.5 \mathrm{mM} \mathrm{CaCl}_{2}$. Adjust $\mathrm{pH}$ to 7.4 with $\mathrm{NaOH}$.

Cytosolic protein isolation buffer

Distilled water with 10mM HEPES, pH7.9, 1.5mM $\mathrm{MgCl}_{2}, 10 \mathrm{mM} \mathrm{KCl}, 10 \mathrm{mM}$ $\mathrm{NaMoO}_{4}, 1 \mathrm{x}$ proteinase inhibitor cocktail, $20 \mathrm{mM} \beta$-glycerophosphate, $2 \mathrm{mM}$ $\mathrm{Na}_{3} \mathrm{VO}_{4}$ and $1 \mathrm{mM}$ PMSF.

Nuclear protein isolation buffer

Distilled water with 20mM HEPES, pH7.9, 1.5mM MgCl $2,420 \mathrm{mM} \mathrm{NaCl}, 10 \mathrm{mM}$ $\mathrm{Na}_{2} \mathrm{MoO}_{4}, 25 \%$ glycerol, $1 \mathrm{x}$ proteinase inhibitor cocktail, $20 \mathrm{mM} \beta-$ glycerophosphate, $2 \mathrm{mM} \mathrm{Na}_{3} \mathrm{VO}_{4}$ and $1 \mathrm{mM} \mathrm{PMSF}$.

Tris-Glycine buffer

Distilled water with $0.3 \%$ Tris, $1.44 \%$ glycine and $20 \% \mathrm{MeOH}$.

PBS-Tween

DPBS with $0.1 \%$ Tween 20 .

With addition of $5 \%$ non-fat milk powder for western blot blocking buffer.

With addition of $2 \%$ BSA for western blot antibody dilution buffer.

Automacs running buffer

DPBS with $1 \%$ heat inactivated FBS and $0.5 \mathrm{mM}$ EDTA. 
ELISA wash buffer

DPBS with $0.05 \%$ Tween 20.

Bioplex buffer

DPBS with $0.1 \%$ BSA, $0.05 \%$ Tween $20,0.005 \%$ sodium azide, $2.5 \mathrm{mM}$ EDTA.

\subsection{Carbonate Buffer}

Distilled water with $85 \mathrm{mM} \mathrm{NaHCO}_{3}$ and $15 \mathrm{mM} \mathrm{Na}_{2} \mathrm{CO}_{3}$, pH to 10.5 with $\mathrm{NaOH}$.

\subsubsection{Preparation of monosodium urate (MSU) crystals}

$45 \mathrm{ml}$ distilled water with $300 \mu \mathrm{l} 5 \mathrm{M} \mathrm{NaOH}$ was heated to gentle boil on a heating/stirring block. Under constant stirring 250mg uric acid was added slowly and stirring was continued until completely dissolved. Volume was adjusted to $50 \mathrm{ml}$ with distilled water and the solution was cooled to $50^{\circ} \mathrm{C}$. The solution was then filtered through a $0.2 \mu \mathrm{m}$ filter and reboiled. Under sterile conditions $1 \mathrm{ml} 5 \mathrm{M} \mathrm{NaOH}$ was added without stirring and the solution was left undisturbed for 7 days at room temperature to allow for crystallisation to occur. Crystals were washed with ethanol then acetone and allowed to dry before being checked for size and shape. MSU crystals were examined by light microscopy and were found to be 5-25um long with a needle like shape and birefringent under polarised light.

\subsubsection{Endotoxin testing}

Endotoxin levels of uric acid, oxonic acid, allopurinol and MSU preparations were determined using the Limulus Amebocyte Lysate assay. For in vitro experiments cells were exposed to $<0.5 \mathrm{EU} / \mathrm{ml}$. For in vivo experiments mice were exposed to $<5 \mathrm{EU} / \mathrm{kg}$. 


\subsection{Human Methods}

\subsubsection{Clinical study design}

15 patients $>18 y$ rs of age with gout as defined by the American Rheumatism Association Preliminary Classification Criteria and the Wallace Criteria ${ }^{156}$ were recruited from the Christchurch Hospital Rheumatology Service. This study was approved by the New Zealand Multi-Region Ethics Committee (MEC/11/06/060) and registered with the Australian and New Zealand Clinical Trials Registry ACTRN 12613000114741. All participants provided written informed consent. The decision to commence allopurinol was made by the treating rheumatologist. The starting dose of allopurinol was $50 \mathrm{mg}$ daily for patients with creatinine clearance $(\mathrm{CrCL})<60 \mathrm{ml} / \mathrm{min}$ and $100 \mathrm{mg} /$ day for patients with a $\mathrm{CrCL}>60 \mathrm{mls} / \mathrm{min}$. The low dose reduces the risk of allopurinol hypersensitivity syndrome and the precipitation of an acute gout flare ${ }^{83}$. Blood was taken directly before starting treatment, one day after the first allopurinol dose and 28 days after starting treatment. At each time point $60 \mathrm{ml}$ of venous blood was taken and processed immediately to isolate blood monocytes (section 2.2.2) for subsequent analyses.

Patient sample size calculation was based on one cytokine, CCL2, as this was the key cytokine of interest. Our previous data indicated an important difference in serum CCL2 is $220 \mathrm{pg} / \mathrm{ml}$ (R Grainger, PhD thesis). The paired standard deviation for repeated measures t-test is not known, nor published in the literature. Therefore, this pilot study could be used to calculate the paired standard deviation of difference in cytokine level before and after uratelowering therapy. The usual sample size for such a study is 12 to 15 subjects.

\subsubsection{Isolation of human blood monocytes}

Blood from the clinical study patients and a separate study involving healthy normouricaemic donors (CEN/08/11/062) was collected by venepuncture into $\mathrm{K}_{3}$-EDTA vacutainers and spun at $840 \mathrm{~g}$ for $10 \mathrm{~min}$ to separate the plasma. Plasma was aspirated, pooled and spun at $840 \mathrm{~g}$ for $10 \mathrm{~min}$. The pellet was discarded and the plasma was heat inactivated at $57^{\circ} \mathrm{C}$ for $30 \mathrm{~min}$, spun at $840 \mathrm{~g}$ for $10 \mathrm{~min}$ to pellet the coagulated proteins and the autologous human 
serum (ahs) was aspirated for preparation of ahsRPMI and subsequent cell culture.

The separated blood cells were diluted two-fold with HBSS and $10-11 \mathrm{ml}$ of this mixture was layered over $3 \mathrm{ml}$ polymorphprep in a $15 \mathrm{ml}$ falcon tube. The blood preparation was then spun at $680 \mathrm{~g}$ for $45 \mathrm{~min}$ with no brake to separate the PBMC (top layer) from the PMN (middle layer) and the RBC (bottom layer). PBMC were aspirated, washed with $40 \mathrm{ml}$ HBSS and spun at $450 \mathrm{~g}$ for $5 \mathrm{~min}$. PBMC where then washed and spun at $300 \mathrm{~g}$ for $10 \mathrm{~min}$ to remove platelets. Residual RBC were lysed by suspending the washed PBMC in 5-10ml RBC lysis buffer and incubating at $37^{\circ} \mathrm{C}$ for $10 \mathrm{~min}$ then PBMC were washed once more in $40 \mathrm{ml} \mathrm{HBSS}$ and spun at $300 \mathrm{~g}$ for $10 \mathrm{~min}$.

Human blood monocytes were isolated from the PBMC using Dynabeads Untouched Human Monocytes. Isolated human blood monocytes were resuspended in ahsRPMI at $2 \times 10^{6}$ cells $/ \mathrm{ml}$ for analysis.

\subsubsection{In vitro monocyte activation assays}

Isolated human blood monocytes were pre-incubated for $1 \mathrm{hr}$ with combinations of UA and the agonists, antagonists or enzymes being investigated then stimulated with LPS $(100 \mathrm{ng} / \mathrm{ml})$ for cytokine production (18hr) or protein expression (up to $1 \mathrm{hr}$ ). Cell density was $1 \times 10^{6} / \mathrm{ml}$.

Cytokine and chemokine production in culture supernatants was analyzed by ELISA or multiplex cytokine array. Protein expression was assessed by western blotting.

\subsubsection{Monocyte photography and cluster analysis}

Isolated human blood monocytes were photographed in flat bottom 96 well plates using an Olympus IX51 inverted microscope (Olympus, Auckland, NZ). Monocyte cluster size was measured using ImageJ software. The area of each cluster within a field of view was estimated using the 'oval' selection tool and the 'measure' function to produce an area measurement. The mean cluster area in each treatment group was then normalised to cluster area of 
LPS alone. The number of monocyte clusters in each field of view for each treatment was also counted.

\subsection{Mouse methods}

\subsubsection{Animal husbandry}

Male C57BL/6 (C57) and Urah ${ }^{\text {Plt/Plt2 }}$ (PLT2) mice were housed in the Biomedical Research Unit, Malaghan Institute of Medical Research (Wellington, NZ) under specific pathogen free conditions with a $12 \mathrm{hr}$ light-dark cycle. Experimental mice were aged between 6-12 weeks. All experimental procedures were undertaken with ethical approval from the Victoria University of Wellington Animal Ethics Committee.

\subsubsection{Mouse model of acute hyperuricaemia}

Oxonic acid $(O A)$ is a uricase inhibitor that prevents the breakdown of UA in the mouse liver ${ }^{157}$. Combining UA with $O A$ will ensure a rapid rise in serum UA and a prolonged state of hyperuricaemia.

OA was suspended in PBS. UA was dissolved in PBS by addition of $10 \mathrm{M}$ $\mathrm{NaOH}$ then the $\mathrm{pH}$ was brought down to 10.5 with $2 \mathrm{M} \mathrm{HCl}$. The UA solution was filter sterilised before administration.

C57 mice were injected i.p. with oxonic acid (300 mg/kg, 30min) followed by i.v. injection of soluble UA (12mg/kg, $1 \mathrm{hr}$ ) (figure $2.1 \mathrm{~A})$.

\subsubsection{Mouse models of urate-lowering therapy (ULT)}

To investigate the effect of lowering serum UA in vivo on systemic inflammation two different methods of ULT were used. Allopurinol was dissolved in carbonate buffer, $\mathrm{pH} 10.5$, by warming to $37^{\circ} \mathrm{C}$. Rasburicase was diluted in PBS. C57 or PLT2 mice were injected i.v. with allopurinol $(30 \mathrm{mg} / \mathrm{kg}$, $3 \mathrm{hr}$ ) or rasburicase $(0.8 \mathrm{mg} / \mathrm{kg}, 3 \mathrm{hr}$ ) (figure $2.1 \mathrm{~B})$. 


\subsubsection{LPS model of systemic inflammation}

To induce a non-lethal systemic inflammatory cytokine response C57 and PLT2 mice were injected i.v. with a low dose of LPS $(40 \mu \mathrm{g} / \mathrm{kg}, 1 \mathrm{hr})$ following induction of acute hyperuricaemia or ULT with allopurinol or rasburicase (figure 2.1).

\subsubsection{Murine blood processing}

Blood was taken from the submandibular facial vein using a $4 \mathrm{~mm}$ lancet (MEDIpoint, Mineola, NY). Blood was collected into microtubes containing $10 \mu \mathrm{l} 0.5 \mathrm{M}$ EDTA and centrifuged immediately at $10,000 \mathrm{~g}$ for $10 \mathrm{~min}$. Plasma was removed and frozen at $-80^{\circ} \mathrm{C}$ for subsequent cytokine and UA measurement.

\subsubsection{Murine spleen processing}

Spleens were dissected from mice, passed through a $40 \mu \mathrm{M}$ cell strainer to create a single cell suspension, washed with $10 \mathrm{ml}$ sfRPMI and spun at $450 \mathrm{~g}$ for $5 \mathrm{~min}$. The cell pellet was resuspended in $5 \mathrm{ml}$ sfRPMI. An aliquot was taken at this stage for analysis of intracellular cytokine production by flow cytometry (section 2.4.2).

\subsubsection{AutoMACS purification of splenic monocytes}

$5 \mathrm{ml}$ of splenic cell suspension was layered over $4 \mathrm{ml}$ Lympholyte $\mathrm{M}$ density gradient and centrifuged for at $1200 \mathrm{~g}$ for $20 \mathrm{~min}$. The upper cell layer was aspirated, washed in PBS and spun at $450 \mathrm{~g}$ for $5 \mathrm{~min}$ then resuspended in $1 \mathrm{ml}$ automacs running buffer. Cells were counted and $1 \mu$ l CD3-biotin and B220biotin per $10^{7}$ cells was added followed by incubation for $15 \mathrm{~min}$ at $4^{\circ} \mathrm{C}$. Cells were then rinsed twice in $5 \mathrm{ml}$ automacs running buffer and spun at $450 \mathrm{~g}$ for $5 \mathrm{~min}$ then resuspended in $200 \mu \mathrm{l}$ automacs running buffer per $10^{7}$ cells. $10 \mu \mathrm{l}$ streptavidin MACS beads were added per $200 \mu$ l then incubated with rolling and tilting for $30 \mathrm{~min}$ at $4^{\circ} \mathrm{C}$. Cells were then rinsed in $5 \mathrm{ml}$ automacs running buffer and spun at $450 \mathrm{~g}$ for $5 \mathrm{~min}$ then resuspended in $2 \mathrm{ml}$ automacs running buffer, passed through a $30 \mu \mathrm{M}$ cell strainer to remove any aggregates and 
run through an AutoMACS (Miltenyi Biotec, Cologne, Germany) to remove CD3 positive and B220 positive cells. The negative fraction was collected and prepared for cytosolic and nuclear protein isolation as described in section 2.5.1.

A Acute Hyperuricaemia

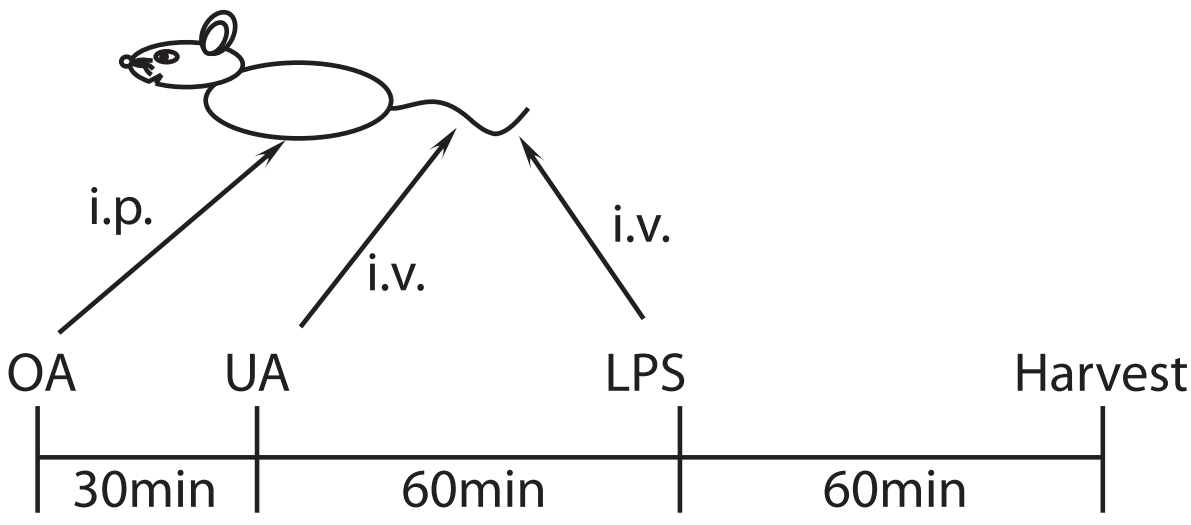

B Urate-lowering therapy

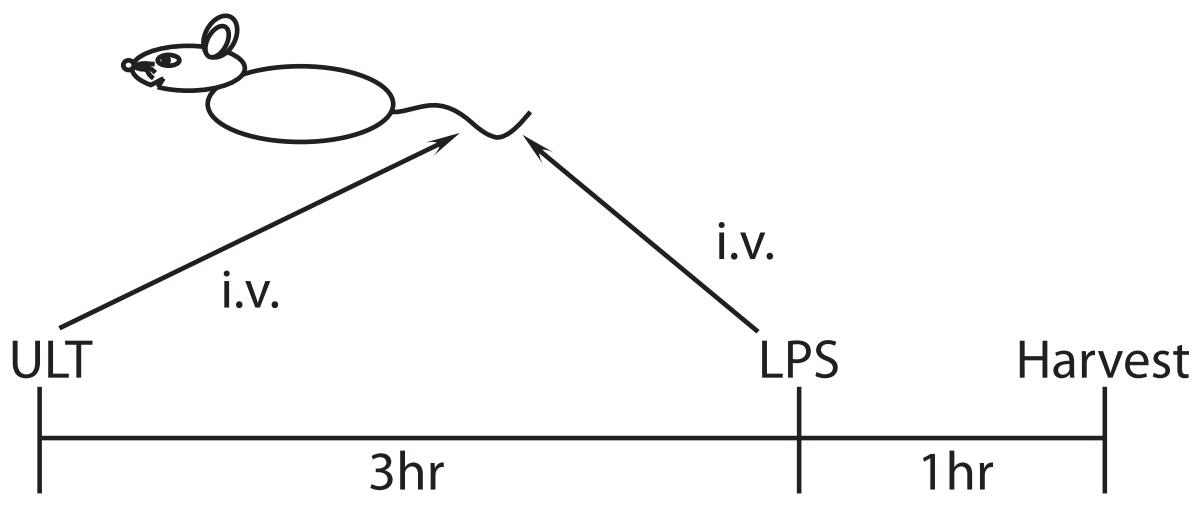

Figure 2.1 Mouse models of acute hyperuricaemia, urate-lowering therapy and systemic inflammation.

A: Mice were injected with OA i.p. (300mg/kg) then UA i.v. $(12 \mathrm{mg} / \mathrm{kg})$ followed by LPS i.v. $(40 \mu \mathrm{g} / \mathrm{kg})$. B: Mice were injected i.v. with allopurinol $(30 \mathrm{mg} / \mathrm{kg})$ or rasburicase $(0.8 \mathrm{mg} / \mathrm{kg})$ followed by $L P S$ i.v. $(40 \mu \mathrm{g} / \mathrm{kg})$. OA = oxonic acid, UA = uric acid, $L P S=$ Lipopolysaccharide, ULT = urate-lowering therapy. 


\subsection{Flow cytometry}

\subsubsection{Surface staining for flow cytometry}

Cells were distributed into 96 well plates for flow cytometry staining then washed with $200 \mu \mathrm{l}$ FACS buffer and spun at $450 \mathrm{~g}$ for $5 \mathrm{~min}$. Blocking antibodies for human (poly-lgG) or mouse (FcyRII clone 2.4G2) were added to prevent non-specific binding, followed by a FACS buffer wash step. Antibody master mixes were added and incubated for 20min. Cells were washed again in $200 \mu$ l FACS buffer then fixed in $4 \%$ neutral buffered formalin for $20 \mathrm{~min}$. Following fixation cells were washed in $200 \mu$ l FACS buffer, then resuspended in $200 \mu$ I FACS buffer for analysis.

\subsubsection{Intracellular staining for flow cytometry}

For analysis of intracellular cytokine production by flow cytometry $10^{6}$ splenocytes were cultured for $4 \mathrm{hr}\left(37^{\circ} \mathrm{C}, 5 \% \mathrm{CO}_{2}\right)$ with GolgiStop (1:1000 dilution) then stained for cell surface markers (section 2.4.1). Following fixation the cells were washed in $200 \mu$ l Saponin buffer to permeabilise and spun at $450 \mathrm{~g}$ for $5 \mathrm{~min}$, then incubated with antibody master mixes prepared in Saponin buffer for 30-60min. The cells were washed in $200 \mu$ l Saponin buffer and spun at $450 \mathrm{~g}$ for $5 \mathrm{~min}$ then resuspended in FACS buffer for analysis.

\subsubsection{Cell death analysis}

Monocytes were washed in $200 \mu \mathrm{l}$ annexin-V binding buffer and spun at $450 \mathrm{~g}$ for 5 min then labelled with the apoptosis marker annexin-V FITC for 20min. Cells were washed again with $200 \mu$ annexin- $V$ binding buffer then resuspended in FACS buffer for flow cytometry. Directly before analysis the cell death marker propidium iodide $(250 \mathrm{ng} / \mathrm{ml})$ was added.

\subsubsection{Intracellular reactive oxygen species}

To measure intracellular production of reactive oxygen species (ROS) monocytes were pre-incubated for $5 \mathrm{~min}$ with the ROS specific dye 
dihydrorhodamine (DHR)-123 $(0.5 \mu \mathrm{M})\left(37^{\circ} \mathrm{C}, 5 \% \mathrm{CO}_{2}\right)$ then stimulated with LPS $(100 \mathrm{ng} / \mathrm{ml})$ or PMA $(5 \mathrm{ng} / \mathrm{ml})$ for $30 \mathrm{~min}$ and analysed by flow cytometry.

\subsubsection{Flow cytometry equipment}

Flow cytometry was performed on a FACSCalibur or LSR-II (BD, Auckland, NZ) and data was analyzed using FlowJo software (Tree Star, Ashland, OR).

\subsection{Western blotting}

\subsubsection{Cytosolic and nuclear protein isolation}

Cytosolic and nuclear protein was isolated from human blood monocytes and murine splenic monocytes using in house cytosolic protein isolation buffer and nuclear protein isolation buffer.

Cells were washed three times in ice cold PBS and all liquid was removed by aspiration. $40-60 \mu \mathrm{l}$ of cytosolic protein isolation buffer was added to each cell pellet and transferred to microcentrifuge tubes for $20 \mathrm{~min}$ on ice. $2.5 \mu \mathrm{l} 10 \%$ igepal was added to each sample then incubated on ice for $2 \mathrm{~min}$. Samples were centrifuged for $2 \mathrm{~min}$ at $4^{\circ} \mathrm{C}, 20,000 \mathrm{~g}$ and the cytosolic protein was removed and stored at $-80^{\circ} \mathrm{C}$. The pellet was then washed with $500 \mu$ ice cold PBS and spun for $2 \mathrm{~min}$ at $4^{\circ} \mathrm{C}, 20,000 \mathrm{~g}$. The PBS was aspirated and the nuclear pellet was resuspended in $20 \mu \mathrm{l}$ nuclear protein isolation buffer and incubated for $30 \mathrm{~min}$ in ice. Samples were spun for $10 \mathrm{~min}$ at $4^{\circ} \mathrm{C}, 20,000 \mathrm{~g}$ and the nuclear protein removed and stored at $-80^{\circ} \mathrm{C}$.

\subsubsection{Determining protein concentration}

The concentration of cytosolic or nuclear protein was measured using the Lowry method ${ }^{158}$ with the Dc Protein Assay Reagent kit. Protein was diluted 3fold in MilliQ water then $5 \mu$ of each sample or BSA standard was aliquoted in duplicate into a flat bottom 96 well plate. A standard curve of $0,0.25,0.5,1,2$ and $4 \mathrm{mg} / \mathrm{ml}$ BSA was used. Dc protein assay reagents $A$ and $S$ were mixed at a $50: 1$ ratio respectively. $25 \mu$ of this mixture was added to each sample and standard well. $200 \mu \mathrm{l}$ Dc protein reagent B was added to each well and mixed 
thoroughly. The reaction was incubated for $15 \mathrm{~min}$ at room temperature then the absorbance was read at $750 \mathrm{~nm}$ with a Versamax microplate reader. Linear regression was used to calculate sample protein concentrations from the standard curve.

\subsubsection{Gel electrophoresis}

Equal amounts of protein (10-20 $\mu$ g) were mixed with MilliQ water, 4x loading buffer and 10x reducing buffer to produce samples of equal volume. These were then heated for $6 \mathrm{~min}$ at $95^{\circ} \mathrm{C}$ in a dry heat block then loaded into NuPAGE Novex 4-12\% Bis-Tris mini gels with Magic Mark and Novex prestained protein ladders as molecular weight markers. The gels were run at $200 \mathrm{~V}$ in MES running buffer for 40-60min. This voltage and running time gave good separation in the $30-70 \mathrm{kD}$ range where the majority of my proteins of interest were.

\subsubsection{Western blotting}

Protein was transferred from the gel to a PVDF membrane $(0.2 \mu \mathrm{m}$ pore size, BioRad, Auckland, NZ) for $2 \mathrm{hr}$ at $300 \mathrm{~mA}$ using a western blot transfer cassette and Tris-Glycine buffer. After transfer membranes were blocked with western blot blocking buffer (PBS-Tween with 5\% non-fat milk powder) for $1 \mathrm{hr}$ then washed with PBS and incubated with primary antibodies diluted in western blot antibody dilution buffer (PBS-Tween with $2 \%$ BSA) overnight at $4^{\circ} \mathrm{C}$. Membranes were washed 3 times with PBS-Tween then incubated with HRP-linked secondary rabbit or mouse antibodies diluted in western blot antibody dilution buffer for $2 \mathrm{hr}$. Membranes were washed 3 times with PBSTween and once with PBS then incubated for 2-5min with Enhanced or Ultra Chemiluminescent Substrate. Membranes were photographed with a Gel Logic 4000 Pro (Carestream, Rochester, NY).

$\beta$-actin was used as a loading control for both cytoplasmic and nuclear protein. $\beta$-actin was compared to Tata Binding Protein (TBP) for nuclear protein loading and found to have an equivalent loading pattern. $\alpha$-tubulin was used 
to test nuclear protein extracts for cytoplasmic protein contamination as $\alpha$ tubulin is not normally present in the nucleus of cells.

\subsubsection{Western blot densitometry}

The density of western blot bands was calculated using ImageJ software. Lanes were selected with the rectangle tool, which produces a peak for each band in the lane. The peaks were closed off with the line tool to remove any background reading and the area of each peak was measured using the wand tool. These area measurements were then converted into a percentage of the total area of the combined peaks using the label peaks function. The percentages of each protein measured were normalised to the control band (day 0 ) then normalised again to the corresponding loading control to produce a measurement relative to day 0 (set at 1) and normalised to the loading control to account for any differences in protein loading.

\subsection{General methods}

\subsubsection{Measurement of serum UA}

Murine serum UA levels were measured using the Uric Acid Detection Kit. $20 \mu$ l serum sample was mixed with $30 \mu$ l UA assay buffer in a 96-well flatbottomed plate. Standards of $0,0.8,1.6,2.4,3.2$ and $4 \mathrm{~nm} /$ well UA diluted in $50 \mu$ l UA assay buffer were set up for each assay. A reaction mixture of $46 \mu l$ UA assay buffer, $2 \mu \mathrm{l}$ UA Probe and $2 \mu \mathrm{l}$ UA Enzyme Mix was prepared for each sample and standard. $50 \mu$ l reaction mixture was added to each sample and standard and the reaction was incubated at $37^{\circ} \mathrm{C}$ for $30 \mathrm{~min}$, protected from light. The fluorescence was measured at $\mathrm{Ex} / \mathrm{Em}=544 / 590$ using a FLUOstar OPTIMA microplate reader (BMG Labtech, Ortenberg, Germany). Linear regression was used to calculate serum UA concentrations from the standard curve.

Healthy human serum UA levels were measured from whole blood using a UASure blood uric acid monitoring system and UASure test strips. For the 
clinical study patient serum UA was measured by Canterbury Health Labs (Christchurch, NZ).

\subsubsection{Chemical assay for anti-oxidant activity}

This assay measures the transfer of electrons from NADH via mPMS to WST1 by measuring the absorbance of reduced WST-1 at 450nm. Anti-oxidant activity of soluble UA can be measured by a decrease in absorbance at $450 \mathrm{~nm}$ compared to control ${ }^{159}$.

A reaction mixture containing 100 $\mu \mathrm{M}$ NADH, $3 \mu \mathrm{M}$ mPMS and $349 \mu \mathrm{M}$ WST-1 was prepared in HBSS in 96well flat bottom plates with the addition of soluble UA $(0.1-0.7 \mathrm{mM})$. SOD $(0.1-1000 \mu \mathrm{M})$ and EGCG $(0.05-50 \mu \mathrm{g} / \mathrm{ml})$ were used as positive controls for anti-oxidant activity. The reaction was carried out for $30 \mathrm{~min}$ at $37^{\circ} \mathrm{C}$ then WST-1 absorbance was measured at $450 \mathrm{~nm}$ with a Versamax microplate reader (Molecular Devices, Sunnyvale, CA).

\subsection{Statistical analysis}

Two-way ANOVA with Bonferroni post-test was used to evaluate significance for time course and titration experiments involving multiple variables. One-way ANOVA with Bonferroni post-test was used when more than two variables were being compared at a single time point. Student's t-test was used when only two variables were used at a single time point. Paired t-test was used to compare clinical study data before and after ULT. Linear regression and Pearson's correlation coefficient were used to evaluate patient data in comparison to their serum UA levels. Statistical analyses were performed with Prism (GraphPad Software, San Diego, CA). 
Chapter 3:

In Vitro Hyperuricaemia 



\section{In vitro hyperuricaemia}

\subsection{Introduction}

The immunological effects of soluble uric acid (UA) on human blood monocytes have not yet been thoroughly investigated.

In other cell types soluble UA has been shown to have stimulatory effects. In vascular smooth muscle cells ${ }^{129}$, adipocytes ${ }^{71}$ and pancreatic $\beta$ cells ${ }^{67}$ soluble UA activates MAP kinase signalling pathways and NFKB family transcription factors. Additionally, the Harper group has shown that human neutrophil superoxide production, following ex vivo monosodium urate (MSU) stimulation, is positively associated with serum UA levels ${ }^{160}$. These studies demonstrate that UA has the ability to modulate signalling pathways that contribute to inflammatory responses.

In a clinical setting, hyperuricaemia correlates with increased monocyte numbers in the blood in patients with gout and asymptomatic hyperuricaemia ${ }^{128}$ and patients with coronary artery disease ${ }^{161}$. However, these studies do not address whether the increase in monocyte numbers translates to differences in monocyte function or disease state. One study shows that monocytes from hyperuricaemic haemodialysis patients have higher rates of apoptosis than healthy controls ${ }^{162}$. This increased monocyte apoptosis is attributed to a direct effect of soluble UA on monocytes as apoptosis is inhibited by the addition of rasburicase, which breaks down the UA.

These studies demonstrate that soluble UA is a ubiquitous regulator of cellular function but there remains a dearth of research into the direct effect of soluble UA on immune cell function. The ability of UA to alter the function of other cell types provides a rationale for investigating the effects of UA on monocyte function.

Given that blood monocytes are directly exposed to soluble UA in the circulation, changes in serum UA could have an immediate effect on monocyte responses to immune challenge. Previously, our group carried out 
an observational clinical study investigating the effect of serum UA levels on circulating innate immune cells in healthy controls versus subjects with hyperuricaemia ${ }^{160}$. One aspect of this study examined the ex vivo response of the participants' blood monocytes following stimulation with LPS. Hyperuricaemia is the main risk factor for gout and gout is a highly inflammatory disease. Based on this relationship we had hypothesised that monocytes from hyperuricaemic participants would be more pro-inflammatory than those from normouricaemic controls. Surprisingly, there was a negative correlation between participants' serum UA levels and production of proinflammatory cytokines by monocytes stimulated ex vivo with LPS (figure 3.1). This negative correlation suggested that UA was having a suppressive effect on monocyte cytokine production. It also provided a rationale to investigate the direct effect of soluble UA, by modelling hyperuricaemia in vitro, on the phenotype and function of human blood monocytes.
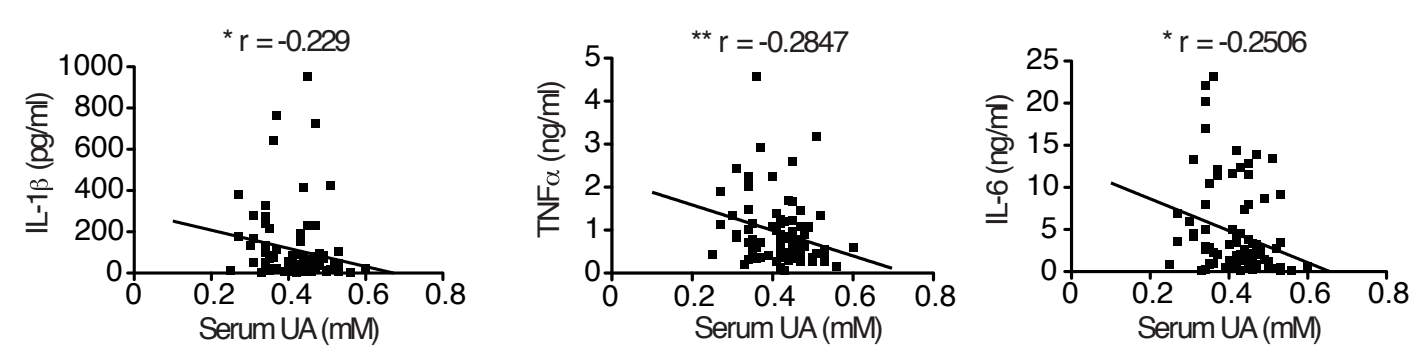

Figure 3.1 Ex vivo monocyte cytokine production negatively correlates with serum UA levels.

Human blood monocytes isolated from participants with hyperuricaemia and normouricaemia $(n=90)$ were stimulated with LPS $(100 \mathrm{ng} / \mathrm{ml}$ ) for $18 \mathrm{hr}$. Cytokine production was measured by multiplex bead array and plotted against participants' serum UA levels. ${ }^{*} p<0.05,{ }^{* *} p<0.01$. Spearman's correlation coefficient.

\subsection{Aim}

The aim of this chapter was to study the direct effect of soluble UA on blood monocyte responses to LPS in vitro and investigate possible mechanisms of action for soluble UA. 


\subsection{Results}

\subsubsection{Monocyte viability and soluble UA}

Apoptosis is a mechanism of programmed cell death that is used by the body to remove cells in a safe and non-inflammatory manner. It has been shown that after a 96hr exposure, soluble UA can increase apoptosis in a human monocytic cell line (U937) and human peripheral blood mononuclear cells ${ }^{162}$. Therefore, it was possible that exposure to high levels of UA in the serum was increasing monocyte cell death in our system, resulting in decreased proinflammatory cytokine production. Therefore, I tested the effect of soluble UA on human monocyte viability at $18 \mathrm{hr}$.

I isolated blood monocytes from healthy, normouricaemic volunteers and stimulated the cells with LPS in the presence or absence of soluble UA for 18hr. I then measured cell viability by flow cytometry using the apoptosis marker annexin $\mathrm{V}$ and the cell death marker propidium iodide. UA had no effect on monocyte viability, defined as annexin $V$ and propidium iodide negative, with or without LPS stimulation (figure 3.2). This suggested that the decrease in monocyte cytokine production in figure 3.1 was not due to increased monocyte cell death, indicating that soluble UA may be suppressing the monocyte cytokine response to LPS.

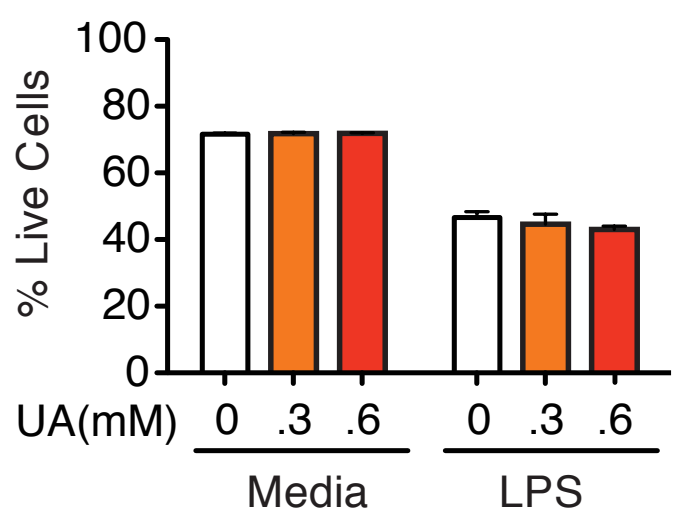

Figure 3.2 Monocyte viability is unaffected by UA.

Human blood monocytes were pre-treated with or without UA (0.3 or $0.6 \mathrm{mM}$ ) for $1 \mathrm{hr}$ then LPS (100ng/ml) for $18 \mathrm{hr}$. Cells were stained with propidium iodide and annexin $V$ and analysed by flow cytometry. Live cells are defined as annexin $V$ negative and $P I$ negative. Results are mean with SEM of triplicate measurements and are representative of two independent experiments from different volunteers. 


\subsubsection{Monocyte cytokine production}

Next I investigated the effect of soluble UA on monocyte cytokine production. To confirm that elevated serum UA could directly suppress the cytokine response of circulating human blood monocytes I treated monocytes from healthy normouricaemic volunteers ex vivo with physiological levels of UA (0.3mM, normal and $0.6 \mathrm{mM}$, hyper). UA alone had no effect on monocyte cytokine production (figure 3.3A-C). In LPS treated monocytes 0.6mM UA decreased IL-1 $\beta$, TNF $\alpha$ and IL-6 compared to LPS alone (figure 3.3A-C). These results showed that soluble UA was having a direct effect by suppressing LPS-induced monocyte cytokine production and confirmed the negative clinical correlation between serum UA levels and monocyte cytokine production.
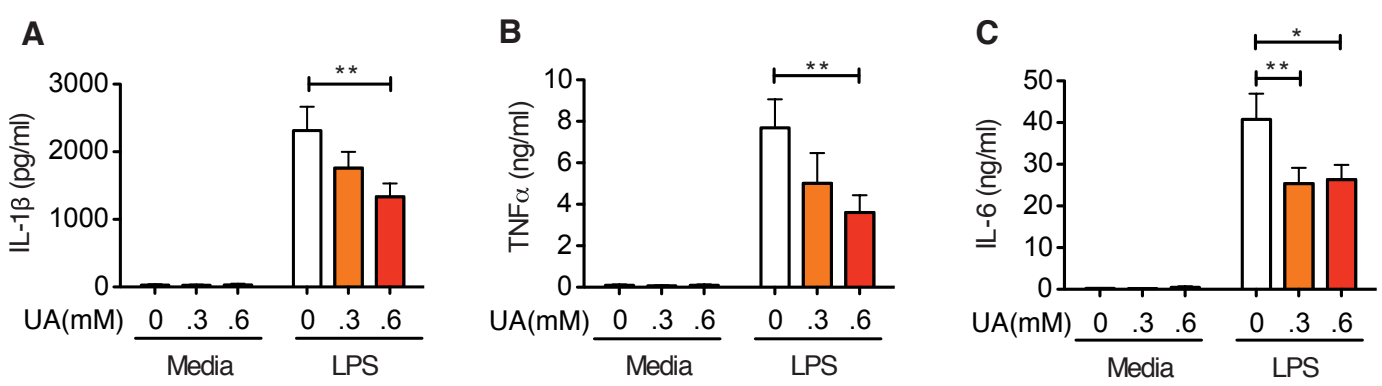

Figure 3.3 UA reduces LPS-induced pro-inflammatory cytokines and enhances IL-10.

Human blood monocytes were pre-treated with or without UA (A-C: $0,0.3$ and $0.6 \mathrm{mM})$ for $1 \mathrm{hr}$ then stimulated with LPS (100ng/ml) for $18 \mathrm{hr}$. A: IL-1 $\beta, B$ : TNF $\alpha$ and C: IL-6 production was assessed by multiplex cytokine bead array. ${ }^{*} p<0.05,{ }^{* *} p<0.01$. Two-way ANOVA with Bonferroni post-test. Results are mean with SEM of triplicate measurements and are representative of five independent experiments from individual volunteers.

\subsubsection{Monocyte LPS signalling machinery}

LPS signals through the LPS receptor complex at the cell surface and two major components of this complex are CD14 and TLR4. CD14 binds LPS in conjunction with LPS binding protein and TLR4 is a DAMP receptor that translates LPS-CD14 binding into the downstream intracellular signalling cascade $^{163}$. I hypothesised that UA could be altering the surface expression of 
CD14 or TLR4 and thereby altering downstream signalling. To test this hypothesis I measured CD14 and TLR4 expression on monocytes after 18hr incubation with UA and/or LPS. LPS enhanced CD14 expression (figure 3.4A) but UA did not change surface expression of CD14 or TLR4 with or without LPS stimulation (figure 3.4A-B). Hence, UA was not altering monocyte cytokine responses via changes to the LPS receptor machinery.

A

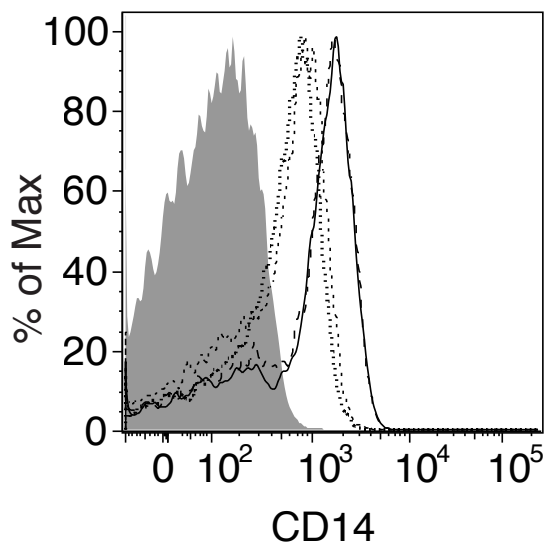

B

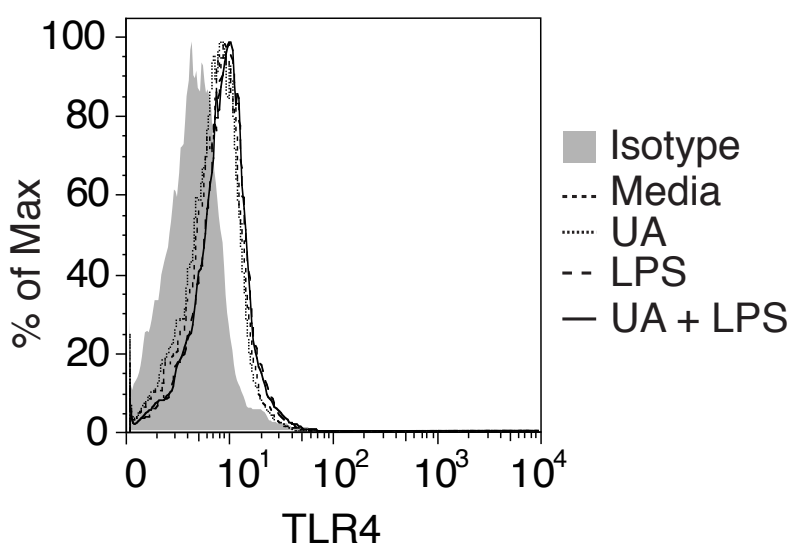

Figure 3.4 CD14 and TLR4 expression is unaffected by UA.

Human blood monocytes were pre-treated with or without UA $(0.6 \mathrm{mM})$ for $1 \mathrm{hr}$ then stimulated with LPS $(100 \mathrm{ng} / \mathrm{ml})$ for $18 \mathrm{hr}$. A: CD14 and B: TLR4 expression was measured by flow cytometry. Results representative of three independent experiments from individual volunteers.

\subsubsection{Intracellular reactive oxygen species (ROS) generation during LPS stimulation}

Next I investigated the intracellular effects of UA on human blood monocytes. Serum UA is currently considered one of the main sinks for circulating $\operatorname{ROS}^{9}$ and reactive nitrogen species (RNS) ${ }^{164,165}$. However, the protective function of soluble UA and its role as an important circulating anti-oxidant is being challenged by more recent publications that report increased serum UA as having an adverse effect on cancer outcomes ${ }^{166}$ and adipocyte homeostasis ${ }^{71}$. ROS can promote inflammatory cytokine production ${ }^{167,168}$. Therefore, I hypothesised that UA was scavenging monocyte intracellular ROS and thereby altering the downstream cytokine signalling. To test the anti-oxidant 
effect of UA I measured intracellular monocyte ROS with dihydrorhodamine123 (DHR-123), which reacts with ROS to produce a fluorescent product that can be measured by flow cytometry. Treatment with UA, LPS or the combination of both did not change the levels of monocyte intracellular ROS (figure 3.5) indicating that UA was not altering cytokine signalling by scavenging intracellular ROS.

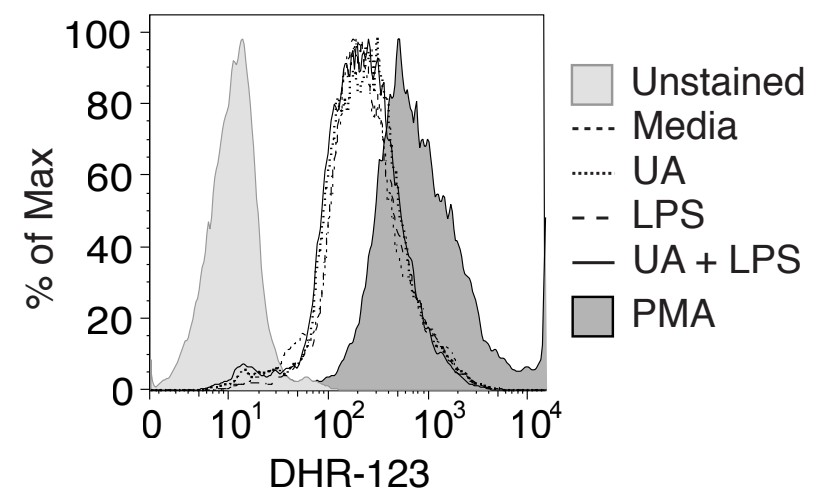

Figure 3.5 Monocyte intracellular ROS is unaffected by UA.

Human blood monocytes were pre-treated with or without UA $(0.6 \mathrm{mM})$ for $1 \mathrm{hr}$ then stimulated for $30 \mathrm{~min}$ with LPS $(100 \mathrm{ng} / \mathrm{ml})$ or PMA $(5 \mathrm{ng} / \mathrm{ml})$. Intracellular ROS production was measured by flow cytometry. Results are representative of three independent experiments from individual volunteers.

\subsubsection{Testing UA as an electron scavenger}

To test the direct anti-oxidant capacity of UA I used a cell free chemical assay where electrons are passed from NADH via methoxy-PMS to a water soluble tetrazolium salt $(\mathrm{WST}-1)^{159}$. The assay measures the oxidation of $\mathrm{NADH}$, through the reduction of WST-1 to a coloured compound, which can be inhibited by anti-oxidants. I measured the reduction of WST-1 in the presence of soluble UA (0.1-0.7mM), using superoxide dismutase (SOD) and the green tea extract epigallocatechin-3-gallate (EGCG), a known dietary anti-oxidant ${ }^{169}$, as positive controls for electron scavenging. UA had no effect on WST-1 reduction at any of the concentrations tested (figure 3.6A), while both SOD and EGCG inhibited the reduction of WST-1 in a concentration dependent manner (figure 3.6B). 
UA had no effect on both intracellular monocyte ROS and a cell free redox assay confirming that the suppression of LPS-induced monocyte cytokines by UA was not mediated by scavenging ROS.
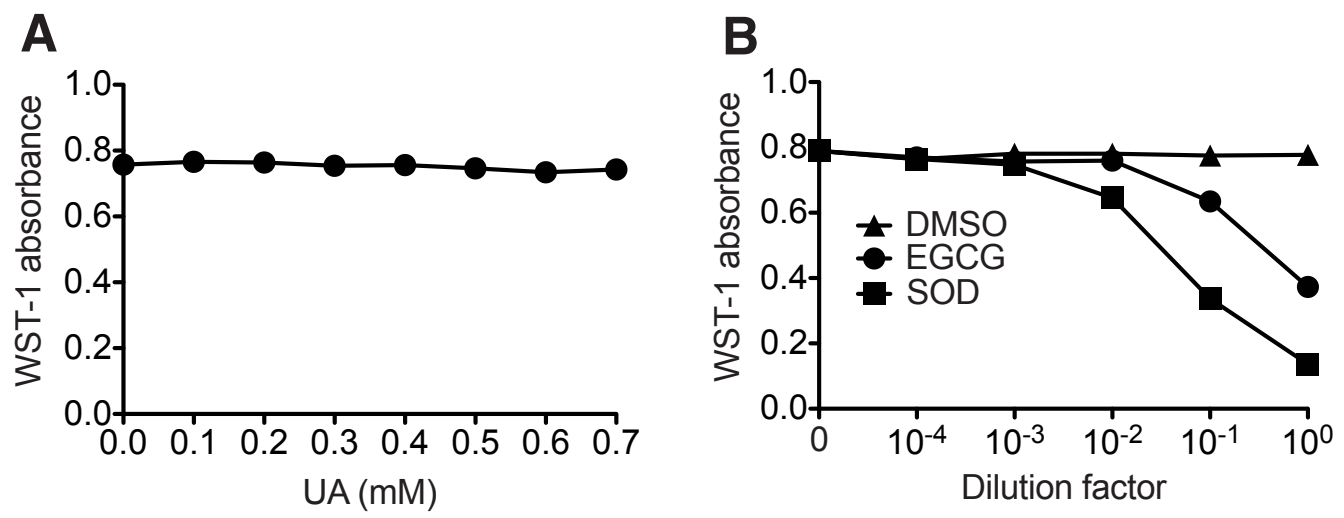

Figure 3.6 Soluble UA is not an electron scavenger.

(A) UA and (B) vehicle (DMSO), EGCG or SOD were titrated into a cell free redox assay measuring the chemical reduction of WST-1. Absorbance was measured at $450 \mathrm{~nm}$. Highest concentrations of EGCG and SOD $\left(10^{\circ}\right)$ were $1000 \mu \mathrm{M}$ and $50 \mu \mathrm{g} / \mathrm{ml}$ respectively. Results are mean with SEM of triplicate measurements and are representative of two independent experiments.

\subsubsection{NFKB signalling pathway}

Downstream of LPS signalling is the NFKB complex, which in monocytes is involved in regulation of cytokine transcription. The NFKB family of transcription factors are important for both pro- and anti-inflammatory cytokine production and function as hetero- and homodimers of the different subunits. (figure 3.7) The p65/p50 heterodimer is a transcription factor for many proinflammatory cytokines while the p50/p50 homodimer is inhibitory of p65/p50 and is itself a transcription factor for IL-10 ${ }^{170,171}$. Concurrent experiments performed by the Harper group (unpublished data) showed that UA had differential effects on nuclear translocation of NFKB subunits in monocytes stimulated with LPS. UA decreased LPS-induced nuclear translocation of NFKB p65 and enhanced early translocation of p50 (appendix 7.1). This indicated a predominance of the p50 subunit and perhaps more p50/p50 homodimer in the nucleus following UA exposure. 


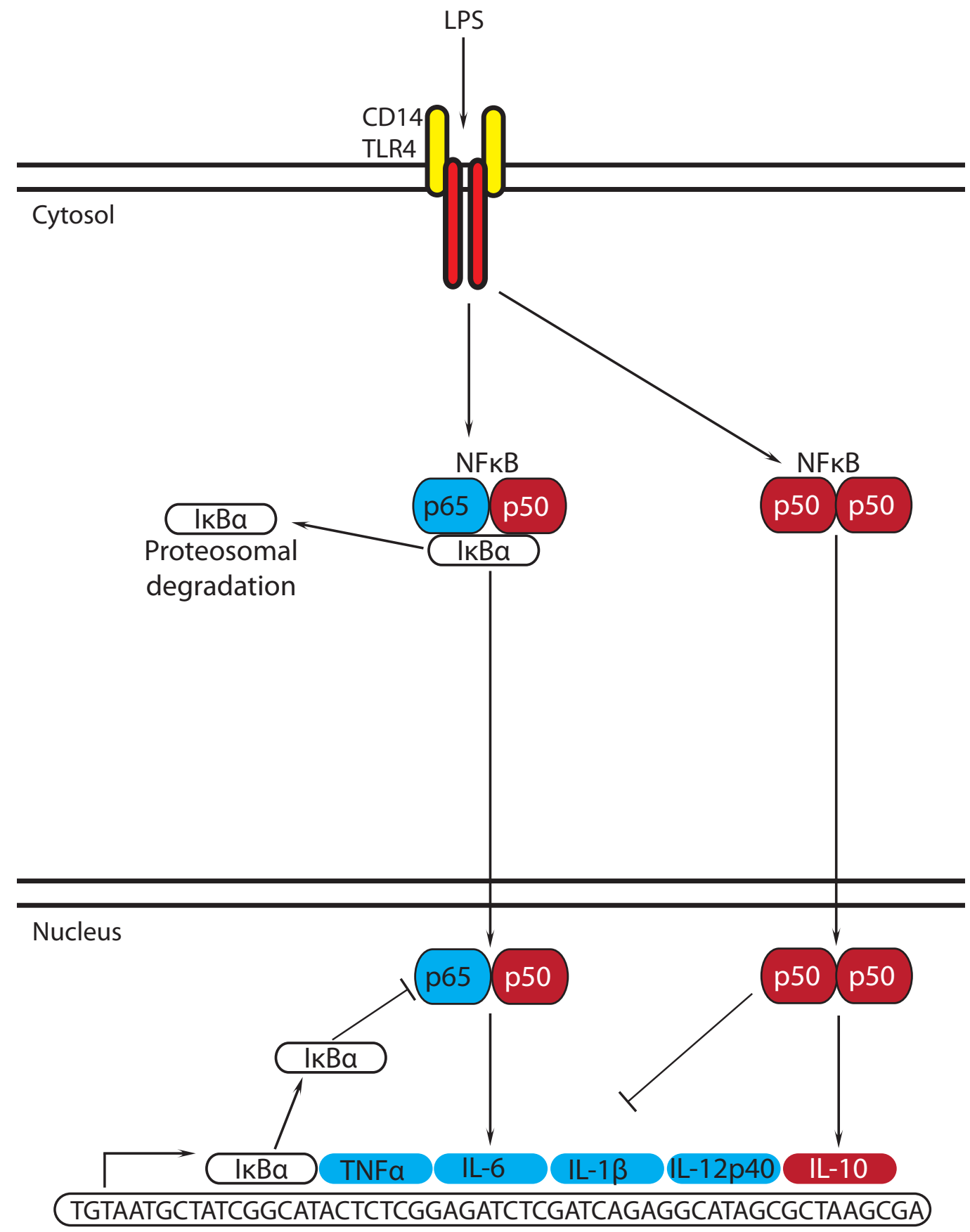

Figure 3.7 LPS-induced monocyte pro- and anti-inflammatory cytokine production via NFKB.

Following LPS stimulation $N F K B \quad p 65 / p 50$ signalling initiates pro-inflammatory cytokine transcription. I $\kappa B \alpha$ production restricts inflammatory $N F \kappa B \quad p 65 / p 50$ signalling and reduces pro-inflammatory cytokine production. NFKB p50/p50 nuclear translocation promotes IL-10 production and inhibits pro-inflammatory cytokines. 
NFкB p65/p50 is sequestered and inactive in the cytoplasm when bound by $I_{\kappa} B \alpha$, which is quickly degraded upon activation by LPS, releasing the p65/p50 heterodimer and allowing it to translocate to the nucleus. IкB $\alpha$ levels are gradually replenished after an initial activation signal to prevent prolonged NFKB activation ${ }^{172}$.

To determine if the reduced nuclear NFKB p65 in the presence of UA was due to altered I $\mathrm{\kappa} B \alpha$ expression I analysed monocyte I $\mathrm{KB} \alpha$ levels by western blot. LPS treatment resulted in a rapid loss of $1 \kappa B \alpha$ levels at $15 \mathrm{~min}$ in both control and UA treated monocytes. However, UA increased the recovery of $1 \kappa B \alpha$ levels at 30 and $60 \mathrm{~min}$ post-LPS (figure 3.8). This indicated that UA could be limiting nuclear translocation of the pro-inflammatory p65/p50 heterodimer via more rapid replenishment of $I_{\kappa} \mathrm{B} \alpha$, after LPS mediated degradation. Altering the p65 to p50 ratio could be one mechanism through which UA inhibited LPS-induced pro-inflammatory cytokine production (figure 3.3).

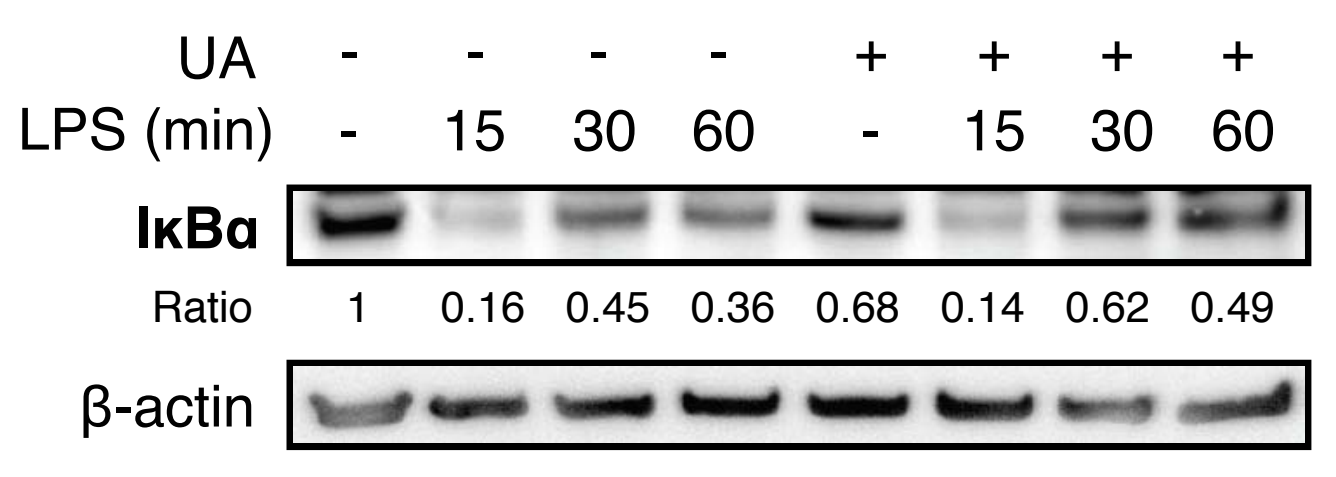

Figure 3.8 $\quad I K B \alpha$ levels return faster with UA treatment after LPS stimulation. Western blot of $I_{\kappa} B \alpha$ from human blood monocytes pre-treated with or without UA $(0.6 \mathrm{mM})$ for $2 \mathrm{hr}$ and 15,30 or $60 \mathrm{~min}$ with LPS $(100 \mathrm{ng} / \mathrm{ml})$. $\beta$-actin was used as a loading control. Results are representative of three independent experiments from individual volunteers. 


\subsubsection{Anti-inflammatory cytokine production}

NFкB p50 nuclear translocation was increased by UA in LPS treated monocytes (appendix 7.1) and the p50/p50 homodimer, while being inhibitory of p65/p50 gene transcription is also a transcription factor for IL-10, a canonical anti-inflammatory cytokine ${ }^{171}$.

Therefore, I wanted to determine whether UA could enhance monocyte IL-10 production. I treated monocytes with UA then stimulated them with LPS and measured production of IL-10 and IL-12p40. UA alone did not induce monocyte IL-10 production but UA and LPS treatment increased monocyte IL10 compared to LPS alone (figure 3.9A). In comparison, treatment with UA decreased production of the pro-inflammatory cytokine IL-12p40 in LPStreated monocytes (figure 3.9B).
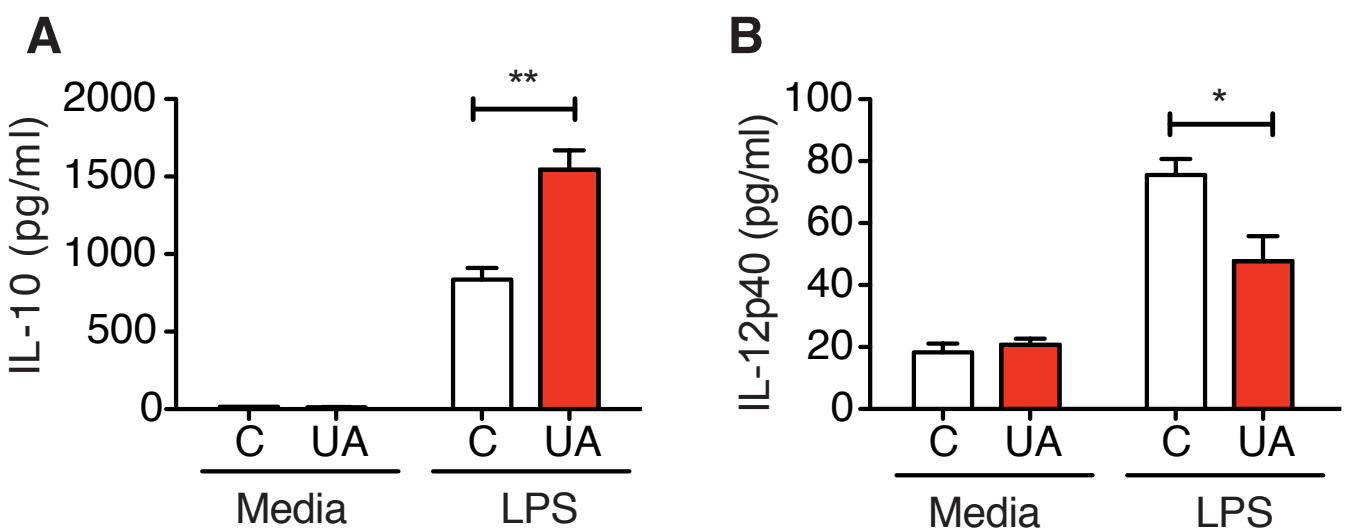

Figure 3.9 UA enhances LPS-induced IL-10 and reduces IL-12p40.

Human blood monocytes were pre-treated with or without $U A(C=0$ and $U A=0.6 \mathrm{mM})$ for $1 \mathrm{hr}$ then stimulated with LPS (100ng/ml) for 18hr. A: IL-10 and B: IL12p40 production in culture supernatant was assessed by ELISA. ${ }^{*} p<0.05,{ }^{* *} p<0.01$. Student's t-test. Results are mean with SEM of triplicate measurements and are representative of six independent experiments from individual volunteers.

\subsubsection{Involvement of MAP kinases and their regulation by UA}

Concurrent research within our group found that monocytes stimulated with LPS in the presence of UA accelerated the dephosphorylation of p38 and ERK1/2 MAP kinases, compared with LPS alone (appendix 7.2). MAP kinase phosphorylation and activity is tightly controlled, in part by the action of dual specific phosphatases (DUSP) ${ }^{173}$ (figure 3.10). 


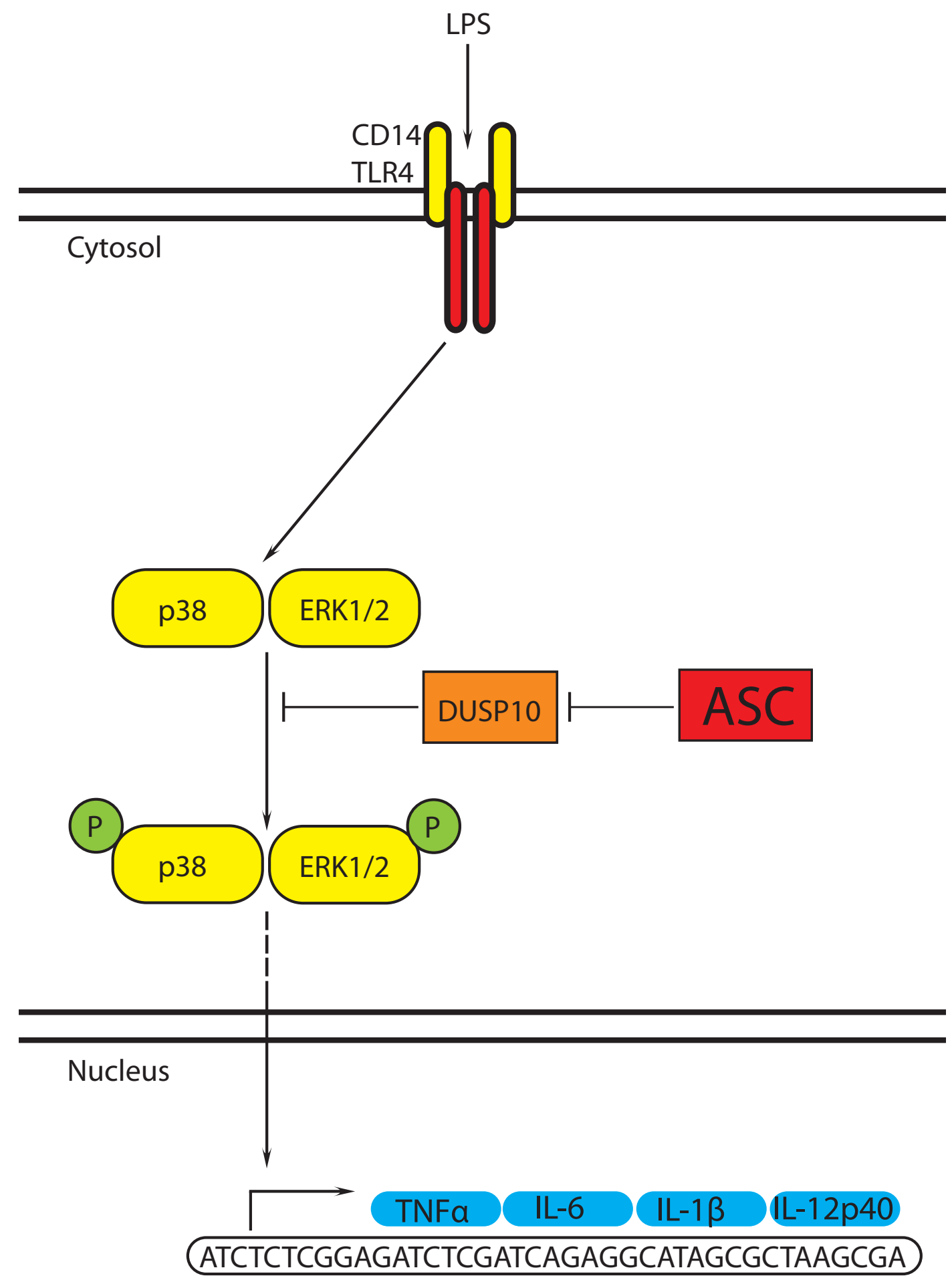

Figure 3.10 LPS-induced monocyte pro-inflammatory cytokine production via MAP kinase signalling.

Following LPS stimulation p38 and ERK1/2 MAP kinases are phosphorylated and activated. DUSP10 can dephosphorylate p38 and ERK1/2 MAP kinase to deactivate them and reduce pro-inflammatory cytokine production but is inhibited by ASC. 
DUSP10 has been shown to be an important homeostatic regulator of LPSinduced inflammation, whereby DUSP10 deficiency results in far greater inflammatory responses and susceptibility to sepsis ${ }^{174}$. In turn, DUSP10 expression is regulated by apoptosis associated speck-like protein containing a CARD (ASC) although the precise mechanism of interaction between DUSP10 and ASC is still unclear ${ }^{175}$. ASC is an adapter protein best characterised for its role in bringing together the NLRP3 inflammasome, the control switch for IL-1 $\beta$ maturation ${ }^{114}$. However, there is increasing evidence that ASC has NLRP3 inflammasome independent functions such as promoting antigen uptake and chemotaxis ${ }^{176}$ and controlling IL-10 production in CD4+ T cells ${ }^{177}$.

Therefore, I investigated monocyte ASC and DUSP10 expression by western blotting to see if these signalling molecules were involved in regulation of the observed MAP kinase dephosphorylation and increased IL-10 production.

I treated monocytes with or without UA for $2 \mathrm{hr}$ and stimulated with LPS for up to $1 \mathrm{hr}$. UA alone reduced monocyte ASC levels and LPS alone reduced monocyte ASC over time (figure 3.11A). DUSP10 levels were increased in UA treated monocytes and decreased following stimulation with LPS in both control and UA treated monocytes (figure 3.11A). I then treated monocytes with UA for 6 and $18 \mathrm{hr}$ and found that UA decreased monocyte ASC at both time points. UA increased DUSP10 at 6 hours but there was no difference at 18 hours (figure $3.11 \mathrm{~B}$ ). 


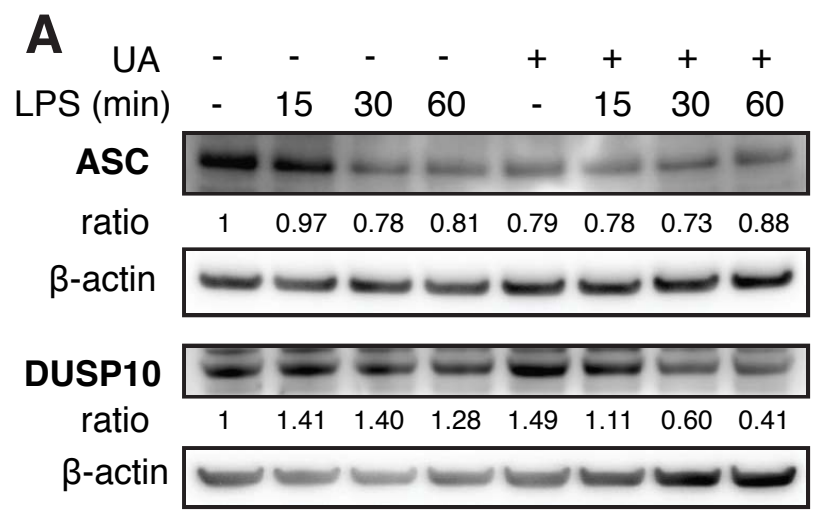

B

$\begin{array}{rcccc}\text { UA } & - & - & + & + \\ \text { Hours } & 6 & 18 & 6 & 18 \\ \text { ASC } & - & - & & \\ \text { ratio } & 1 & 1.86 & 0.57 & 0.87 \\ \text { DUSP10 } & - & & & \\ \text { ratio } & 1 & 1.61 & 1.45 & 1.71 \\ \text { B-actin } & -\end{array}$

Figure 3.11 UA treatment reduces ASC and increases DUSP10 expression. Western blots of ASC and DUSP10. A: Human blood monocytes were pre-treated with or without UA $(0.6 \mathrm{mM})$ for $2 \mathrm{hr}$ and 15,30 or $60 \mathrm{~min}$ with LPS $(100 \mathrm{ng} / \mathrm{ml})$. B: Human blood monocytes were treated for $6 \mathrm{hr}$ or $18 \mathrm{hr}$ with or without UA (0.6mM). $\beta$ actin was used as a loading control. Results are representative of two to six independent experiments from individual volunteers.

In this instance UA had a measurable effect in the absence of LPS stimulation. This implicated a reduction in ASC levels and concomitant increase in DUSP10 expression (figure 3.11) as the initial event that was suppressing the monocyte inflammatory cytokine response to LPS. The enhancement of IL-10 production appears to be a result of UA dependent changes to NFKB signalling (figure 3.8).

\subsubsection{Adenosine receptors and purine recognition}

Adenosine has been shown to have differential effects on leukocyte function, such as mediating chemotaxis ${ }^{178}$, reducing inflammatory cytokine production and enhancing IL-10 ${ }^{179,180}$, through the four adenosine receptors subtypes: $A 1$, A2a, A2b and A3. All four receptor subtypes are present on monocytes ${ }^{181}$. Adenosine is the main physiological agonist of adenosine receptors and caffeine is an environmental non-selective antagonist ${ }^{182}$. UA shares the common core purine structure with adenosine and caffeine (figure 3.12$)^{183}$. Therefore, UA could be signalling through an adenosine receptor to enhance LPS-induced IL-10 production. 
<smiles>O=c1[nH]c2c(c(=O)[nH]1)NCN2</smiles>

Uric Acid<smiles>Nc1ncnc2c1ncn2[C@@H]1O[C@H](CO)[C@@H](O)[C@H]1O</smiles>

Adenosine<smiles>Cn1c(=O)c2c(ncn2C)n(C)c1=O</smiles>

Caffeine

Figure 3.12 Chemical structures of uric acid, adenosine and caffeine highlighting their core purine structure.

\subsubsection{The role of adenosine receptors in UA-enhanced monocyte IL-10 production}

Considering the structural similarity between UA, adenosine and caffeine I tested the hypothesis that UA was enhancing LPS-induced IL-10 by signalling through one of the four adenosine receptors.

I pre-treated monocytes with UA and known adenosine receptor antagonists to block the four receptors individually: SLV320 (A1), ZM241385 (A2a), PSB1115 (A2b) and VUF5574 (A3). I then stimulated with LPS and measured monocyte cytokine production.

The $A 1, A 2 b$ and $A 3$ receptor antagonists had no effect on the increased IL10 production caused by UA in LPS-stimulated monocytes (figure 3.13A, C and D). The A2a antagonist had no effect on LPS alone treated monocytes but inhibited LPS-induced IL-10 production in UA treated monocytes in a concentration dependent manner (figure 3.13B).

This suggested that UA could be signalling through the $A 2 a$ receptor to enhance production of IL-10 by monocytes stimulated with LPS. 

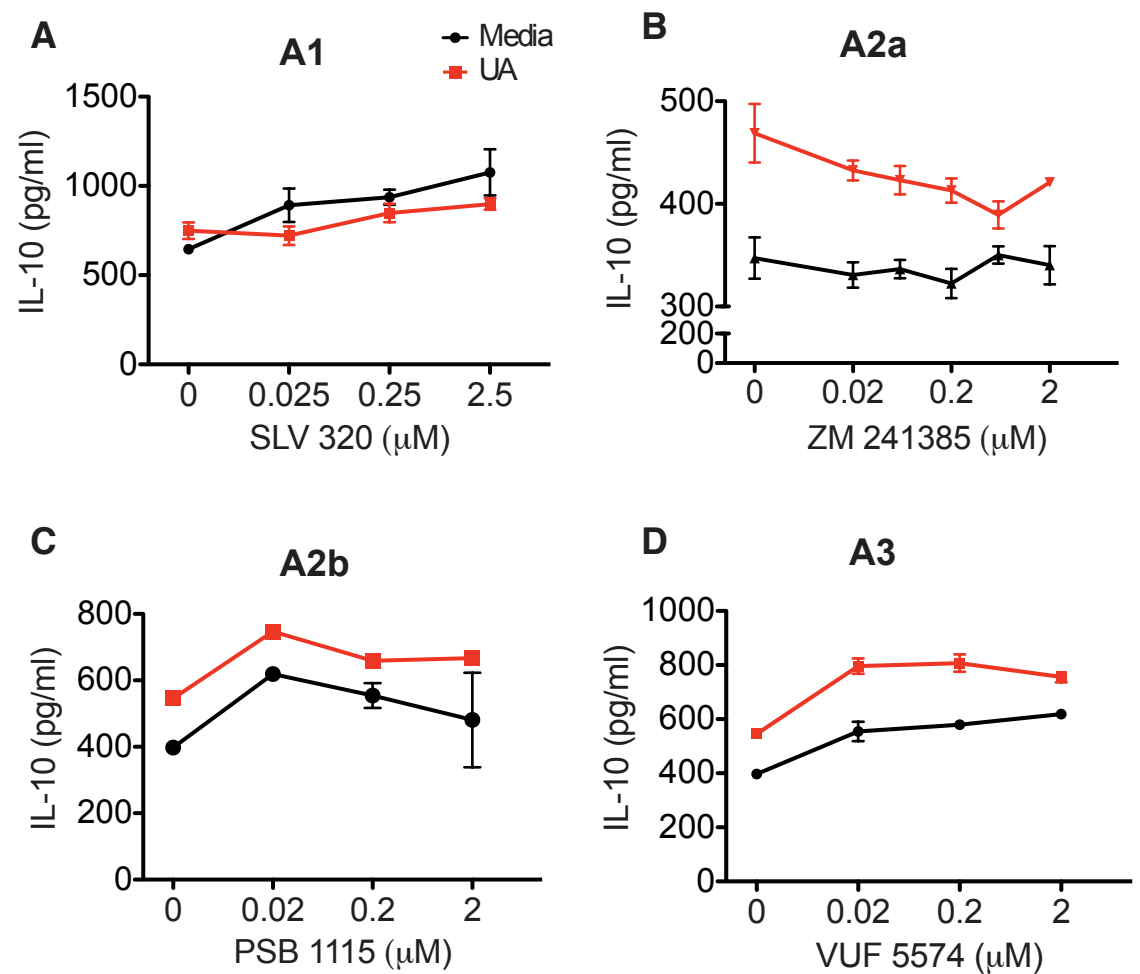

Figure 3.13 A2a receptor antagonist inhibits the IL-10 increase caused by UA. Human blood monocytes were pre-treated with or without UA $(0.6 \mathrm{mM})$ and adenosine receptor antagonists for $1 \mathrm{hr}$ then stimulated with LPS $(100 \mathrm{ng} / \mathrm{ml})$ for $18 \mathrm{hr}$. A: SLV320 (A1), B: ZM241385 (A2a), C: PSB1115 (A2b) and D: VUF5574 (A3) IL-10 production was measured by ELISA. Results are mean with SEM of triplicate measurements and are representative of two independent experiments from individual volunteers.

Adenosine can be released by monocytes in an autocrine-signalling loop that enhances LPS-induced IL-10 via adenosine A2a receptor signalling ${ }^{184}$. Therefore, I investigated whether adenosine was involved in the UA-mediated increase in LPS-induced IL-10. I treated monocytes with adenosine deaminase (ADA) and/or the $\mathrm{A} 2 \mathrm{a}$ receptor antagonist followed by adenosine or UA and then measured monocyte IL-10 production after LPS stimulation.

There was no effect of adenosine, UA or ADA on IL-10 production in the absence of LPS stimulation (figure 3.14A-B). Both ADA and the A2a antagonist treatment decreased the basal level of LPS-induced IL-10, confirming a role for endogenous adenosine in increasing monocyte IL-10 production (figure 3.14 white bars). Adenosine potently enhanced LPSinduced IL-10 and this enhancement was blocked by ADA, the A2a antagonist 
and the two combined. However, adenosine still moderately increased LPSinduced IL-10 in the presence of the A2a receptor antagonist (figure 3.14A, brown bars). UA increased LPS-induced IL-10 production over LPS alone and this increase was not inhibited by ADA, the $A 2 a$ antagonist or the two combined (figure 3.14B, red bars).
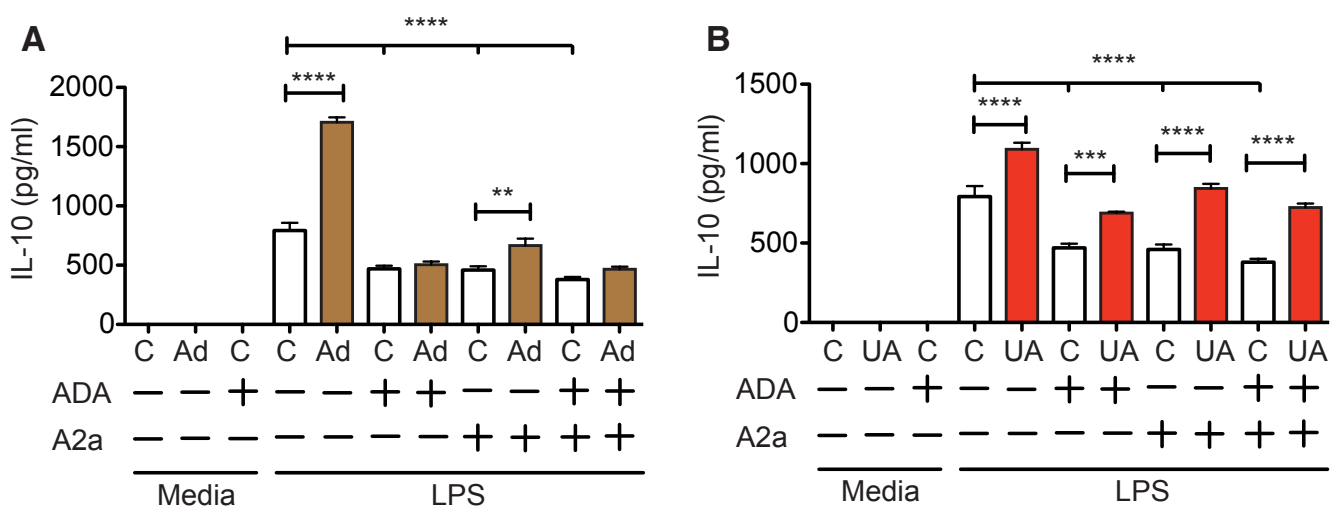

Figure 3.14 UA increases monocyte IL-10 independently of adenosine and A2a receptor signalling.

A: Human blood monocytes were pre-treated with or without Ad (adenosine, $0.1 \mathrm{mM}$ ) or B: UA (0.6mM), ADA (adenosine deaminase $3 \mathrm{U} / \mathrm{ml}$ ), A2a (A2a receptor antagonist, $20 \mu \mathrm{M})$ for $1 \mathrm{hr}$ then stimulated with LPS $(100 \mathrm{ng} / \mathrm{ml})$ for $18 \mathrm{hr}$. IL-10 production was measured by ELISA. ${ }^{* *} p<0.01,{ }^{* * *} p<0.001,{ }^{* * * *} p<0.0001$. One-way ANOVA with Bonferroni post-test. Results are mean with SEM of triplicate measurements and are representative of three independent experiments from individual volunteers.

These results showed that both $\mathrm{ADA}$ and the $\mathrm{A} 2 \mathrm{a}$ antagonist inhibited endogenous and exogenous adenosine from enhancing LPS-induced IL-10. However, $A D A$ and the $A 2 a$ antagonist were unable to prevent UA from enhancing monocyte IL-10 production. Thus, it appeared that UA was not enhancing LPS-induced IL-10 by increasing adenosine release from monocytes.

Interestingly, adenosine increased LPS-induced IL-10 in the presence of the A2a antagonist suggesting an increased capacity for adenosine to signal via the $A 2 b$ receptor when the $A 2 a$ receptor was blocked ${ }^{185}$. 


\subsubsection{1 $\mathrm{A} 2 \mathrm{a}$ and $\mathrm{A} 2 \mathrm{~b}$ receptor signalling and protein kinase $\mathrm{A}$ (PKA)}

The $A 2 a$ and $A 2 b$ adenosine receptors have the same downstream signalling pathway ${ }^{186}$. Consequently, blocking the A2a receptor could potentially shift UA signalling from the $A 2 a$ to the $A 2 b$ receptor.

To test whether UA could be signalling through the $A 2 b$ receptor I measured IL-10 release from monocytes treated with combinations of UA and the A2a and $A 2 b$ receptor antagonists then stimulated with LPS. Blockade of both the $\mathrm{A} 2 \mathrm{a}$ and $\mathrm{A} 2 \mathrm{~b}$ receptors individually and combined did not prevent UA from enhancing monocyte IL-10 production after LPS stimulation (figure 3.15A).

Signalling through the $A 2 a$ or $A 2 b$ receptors activates protein kinase $A(P K A)$; an intermediate kinase in the canonical cAMP-signalling pathway downstream of adenosine $A 2 a$ and $A 2 b$ receptors ${ }^{187}$. To see whether blocking PKA could prevent UA from augmenting LPS-induced IL-10 I treated monocytes with a specific PKA inhibitor (KT 5270) in combination with UA, then stimulated them with LPS.

The PKA inhibitor reduced IL-10 production from LPS only treated monocytes but did not prevent UA from increasing LPS-induced IL-10 (figure 3.15B).

Collectively these results showed that UA was increasing the production of IL10 from LPS-stimulated monocytes independently of both adenosine release and direct activation of the adenosine $\mathrm{A} 2 \mathrm{a}$ and $\mathrm{A} 2 \mathrm{~b}$ receptors. 
A

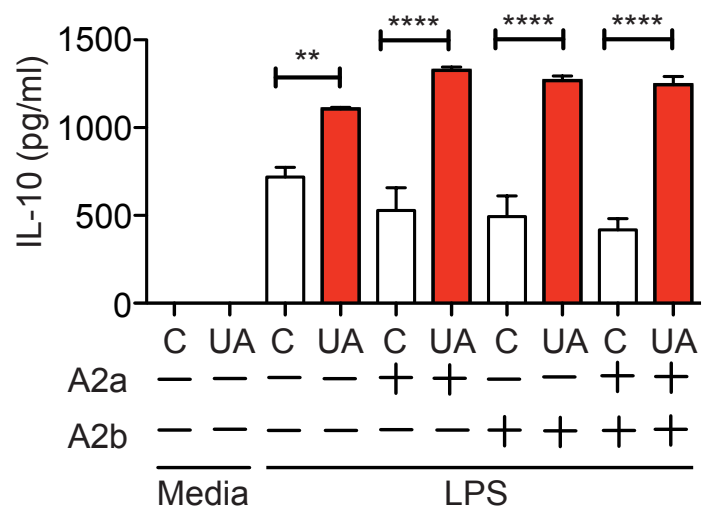

B

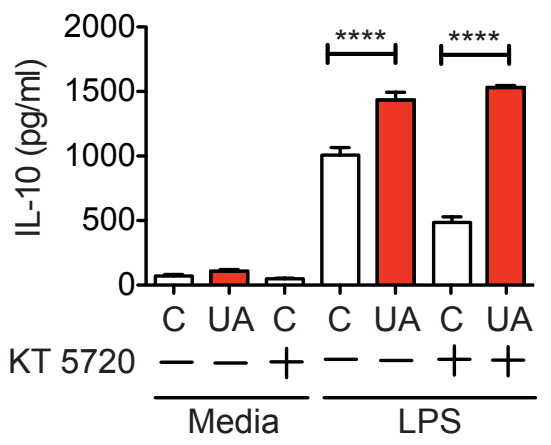

Figure 3.15 UA does not signal through the A2a or $A 2 b$ receptor.

A: Human blood monocytes were pre-treated with or without UA (0.6mM), A2a (A2a receptor antagonist, 20 $\mu \mathrm{M}), A 2 b(A 2 b$ receptor antagonist, 100nM) for $1 \mathrm{hr}$ then stimulated with LPS (100ng/ml) for 18hr. B: Human blood monocytes pre-treated with or without UA (0.6mM) and KT 5720 (PKA inhibitor, $1 \mu \mathrm{M})$ for $1 \mathrm{hr}$ then stimulated with LPS $\left(100 \mathrm{ng} / \mathrm{ml}\right.$ ) for $18 \mathrm{hr}$. IL-10 production was measured by ELISA. ${ }^{* *} p<0.01$, ${ }^{* * * *} p<0.0001$. One-way ANOVA with Bonferroni post-test. Results are mean with SEM of triplicate measurements and are representative of two independent experiments from individual volunteers.

\subsubsection{Monocyte homotypic aggregation}

Studies of human monocytic U937 cells have shown that activation causes homotypic aggregation ${ }^{188,189}$.

While investigating monocyte cytokine production I observed that LPS treatment caused the cells to aggregate together in small clusters and UA increased the size of LPS-induced clusters (figure 3.16C-D). UA alone did not induce monocyte aggregation (figure 3.16A-B). I also measured the size of each monocyte cluster using ImageJ software, normalising to LPS alone, and counted the number of clusters in each treatment. UA treatment increased average cluster size and there was a trend towards a decrease in the total number of clusters in LPS-stimulated monocytes (figure 3.16E-F). There was a reciprocal relationship between cluster size and the number of clusters suggesting that monocytes were migrating together to form the clusters.

Adhesion and migration are important monocyte functions. This led me to investigate UA's effect on monocyte aggregation, in parallel with the cytokine production studies detailed earlier, to see if there was a common mechanism involved in both aggregation and cytokine production. 
C

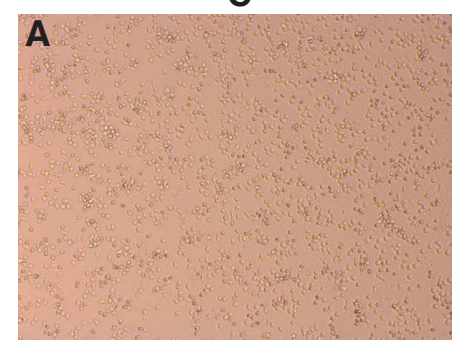

LPS

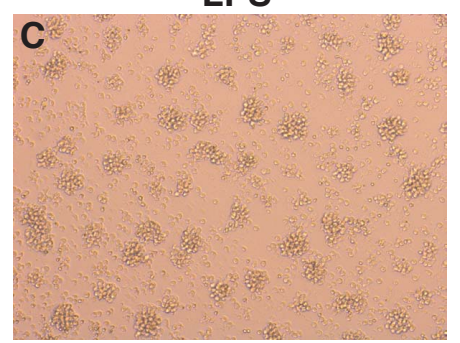

UA
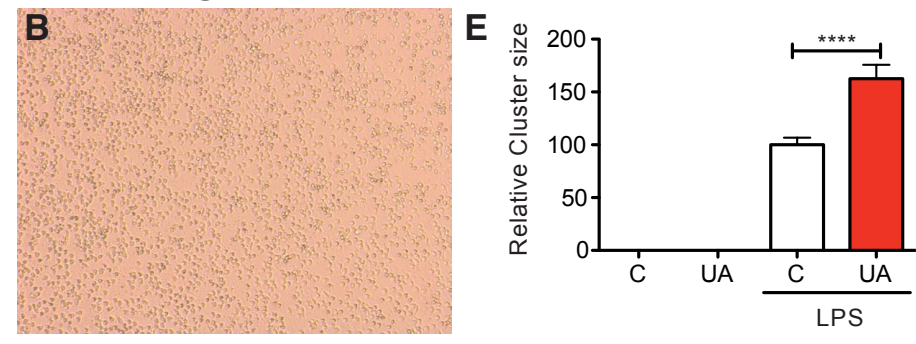

LPS + UA
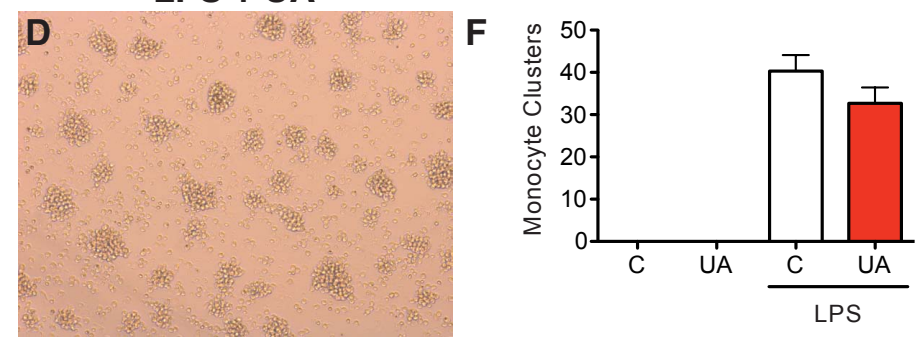

Figure 3.16 Monocyte aggregation is enhanced by UA.

A-D: Human blood monocytes were pre-treated with or without UA $(0.6 \mathrm{mM})$ for $1 \mathrm{hr}$ then stimulated with LPS $(100 \mathrm{ng} / \mathrm{ml})$ for $18 \mathrm{hr}$. Results are representative of three independent experiments from individual volunteers. E: Average cluster size, relative to LPS alone. F: Cluster number. ${ }^{* * * *} p<0.0001$, student's $t$-test. Results are mean with SEM of triplicate measurements and are representative of three independent experiments from individual volunteers.

\subsubsection{Monocyte aggregation and adenosine receptor activation}

Signalling via the adenosine receptors regulates cytokine production in activated monocytes ${ }^{180}$ but also alters chemokine receptor and adhesion molecule expression ${ }^{178}$. Therefore, I hypothesised that UA was signalling through adenosine receptors to enhance LPS-induced monocyte homotypic aggregation.

To study adenosine receptor involvement in monocyte homotypic aggregation I compared the effect of UA and adenosine on LPS-induced monocyte aggregation and found that both UA and adenosine increased aggregation of LPS treated monocytes (figure 3.17A-C). I then used adenosine receptor antagonists to block the four adenosine receptors individually. The $A 1, A 2 b$ and $\mathrm{A} 3$ receptor antagonists had no effect, while the $\mathrm{A} 2 \mathrm{a}$ receptor antagonist potently inhibited LPS-induced monocyte aggregation (figure 3.17, left column). The $\mathrm{A} 2 \mathrm{a}$ receptor antagonist also inhibited monocyte aggregation in the presence of UA and adenosine (figure 3.17G-I). 


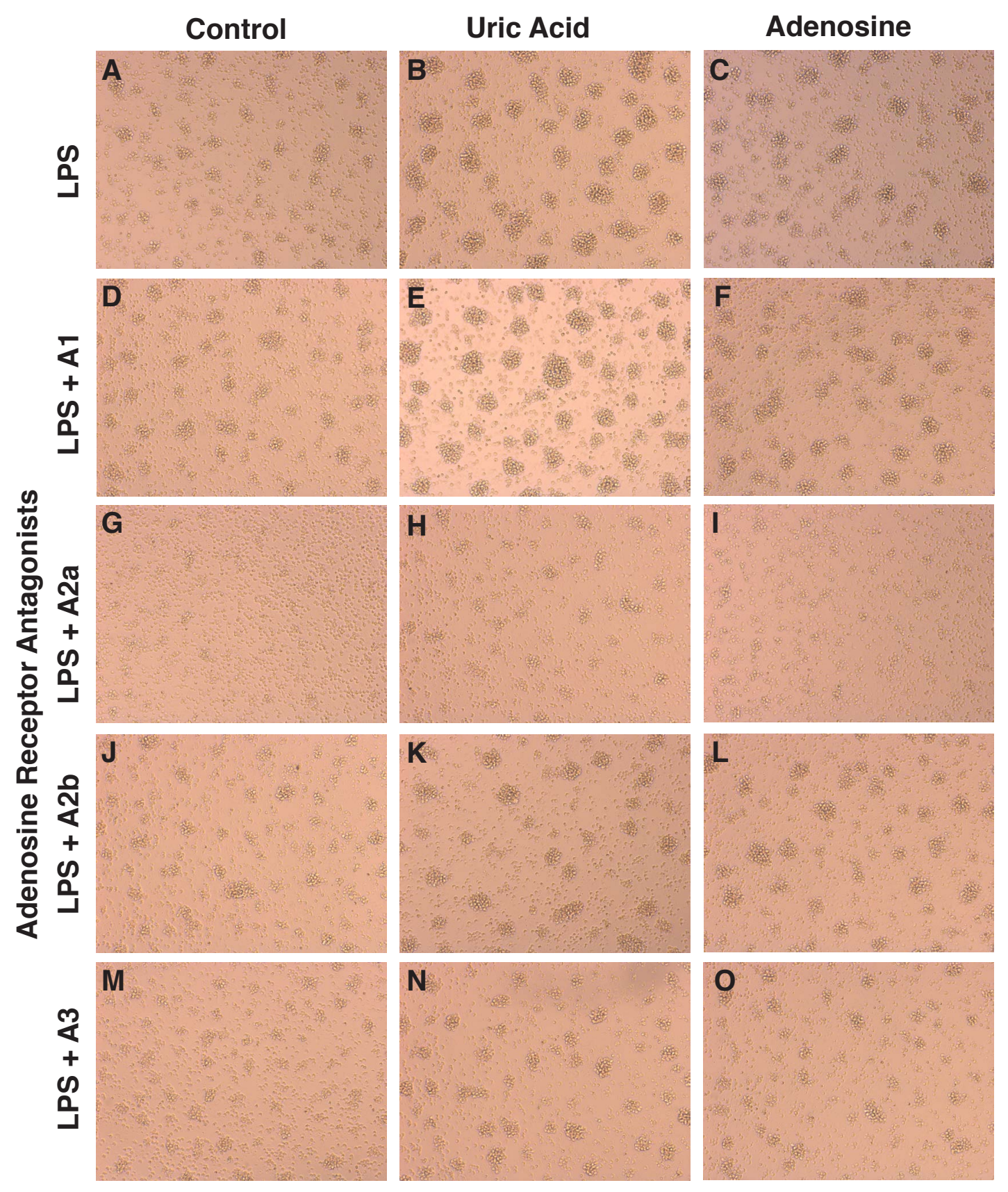

Figure 3.17 Monocyte aggregation is inhibited by the A2a receptor antagonist. A-O: Human blood monocytes were pre-treated with or without UA (0.6mM) or adenosine $(0.1 \mathrm{mM})$ and adenosine receptor antagonists for $1 \mathrm{hr}$ then stimulated with LPS (100ng/ml) for 18hr. Adenosine receptor antagonist concentrations were: A1 (5nM), A2a (20 $\mu \mathrm{M}), A 2 b(100 \mathrm{nM})$ and $A 3(40 \mathrm{nM})$. Results are representative of two independent experiments from individual volunteers. 
Figure 3.18A shows again that UA and adenosine both increased the size of LPS-induced monocyte clusters and demonstrated that UA increased cluster size when combined with the different receptor antagonists. However, there was no clear relationship between adenosine receptor antagonists and the number of clusters (figure 3.18B).
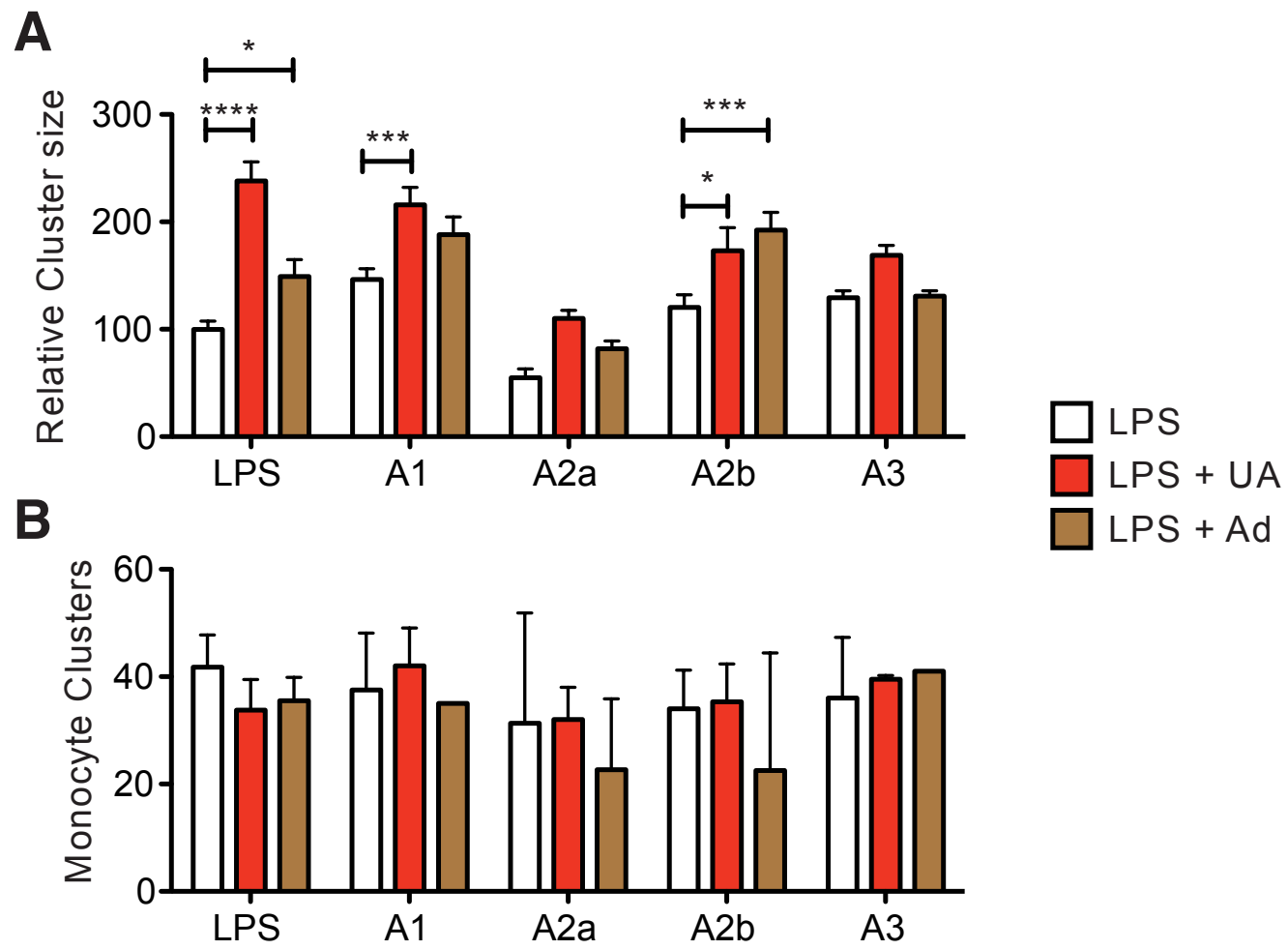

Figure 3.18 Monocyte aggregation, but not cluster formation, is inhibited by the $A 2 a$ receptor antagonist.

Monocytes were treated as in figure 3.17. A: Monocyte cluster size relative to LPS alone. B: Average number of clusters in each field of view. ${ }^{*} p<0.05,{ }^{* *} p<0.001$, ${ }^{* * * *} p<0.0001$, Two-way ANOVA with Bonferroni post-test. Results are mean with SEM of A: total clusters per field of view and are representative of three independent experiments from individual volunteers and B: total number of clusters and are combined from three independent experiments from individual volunteers. 
As discussed earlier adenosine can be released from activated monocytes to feed back in an autocrine manner ${ }^{184}$ and treatment with the $A 2 a$ antagonist alone reduced LPS-induced monocyte aggregation (figure 3.17A vs. G). This suggested a role for endogenous monocyte derived adenosine in mediating LPS-induced aggregation and UA may simply be increasing adenosine release and subsequently increasing aggregation.

To investigate the relationship between of UA and adenosine in mediating monocyte homotypic aggregation I treated monocytes with adenosine deaminase (ADA) to remove adenosine from the culture system. Monocytes pre-treated with ADA, then stimulated with LPS formed smaller clusters than when treated with LPS alone (figure 3.19A vs. D). Combined treatment with $A D A$ and the $A 2 a$ antagonist also resulted in decreased LPS-induced monocyte aggregation (figure 3.19A vs. J). UA enhanced LPS-induced aggregation in the presence of ADA (figure 3.19D vs. E), the A2a antagonist (figure 3.19G vs. $\mathrm{H}$ ) and the two combined (figure 3.19J vs. K). Adenosine did not enhance LPS-induced monocyte aggregation when ADA, the A2a antagonist or the two combined were present (figure 3.19G, I, J and L).

Measuring cluster size shows graphically that UA enhanced LPS-induced monocyte aggregation regardless of ADA or A2a antagonist treatment. Adenosine also increased cluster size but this increase was removed by treatment with either ADA or the A2a antagonist (figure 3.20A). Again there was no clear pattern present when comparing the number of clusters in each treatment group (figure 3.20B).

These results showed that the release of endogenous adenosine from monocytes was important for potentiating aggregation during LPS stimulation. As with the LPS-induced monocyte cytokine production UA was not enhancing adenosine release or signalling directly through the A2a receptor to increase monocyte aggregation. 

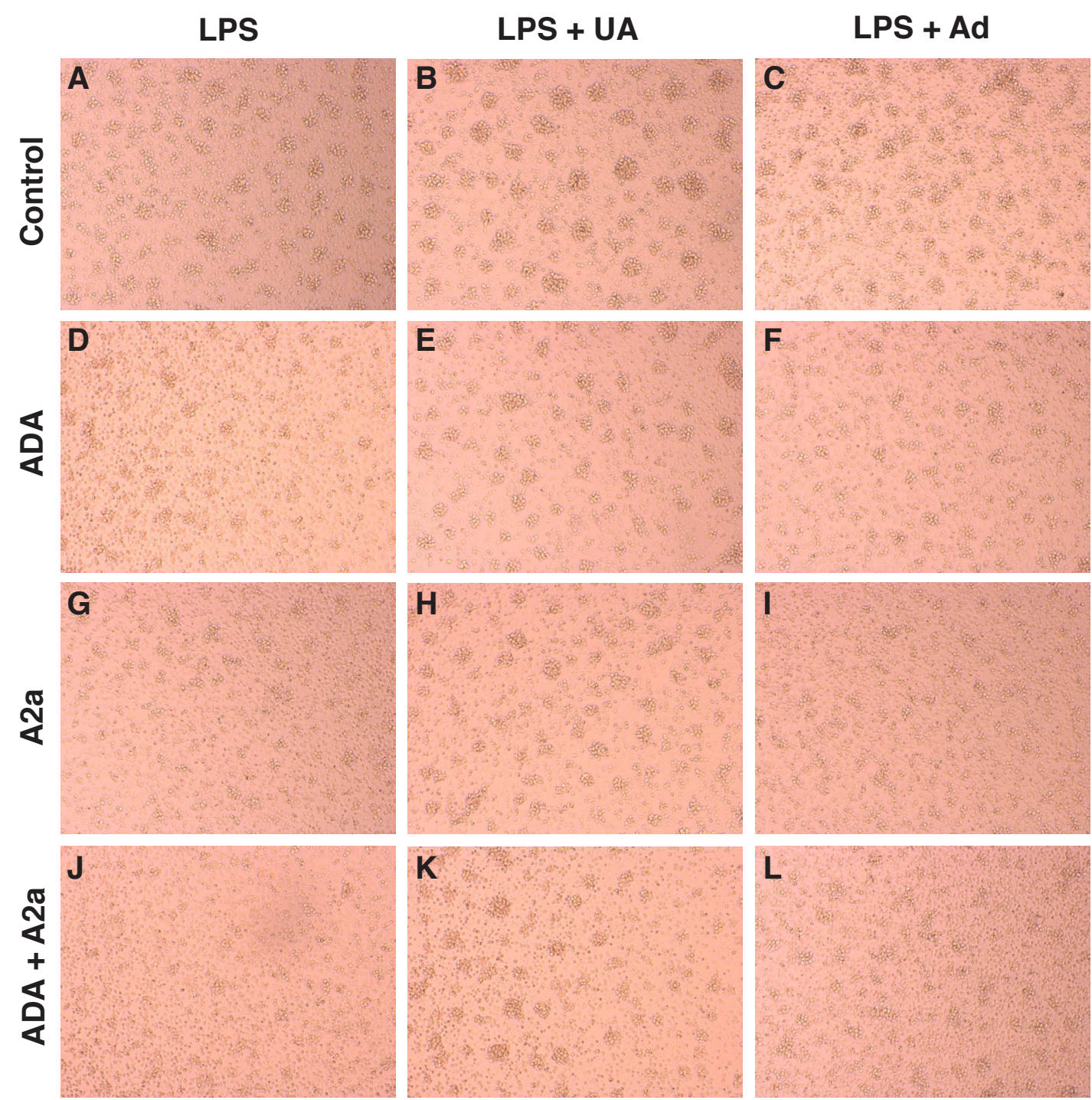

Figure 3.19 UA enhances LPS-induced aggregation independently of adenosine signalling.

A-L: Human blood monocytes were pre-treated with or without UA $(0.6 \mathrm{mM})$ or adenosine $(0.1 \mathrm{mM})$ plus $A D A(3 \mathrm{U} / \mathrm{ml})$ and/or $A 2 \mathrm{a}$ antagonist $(10 \mu \mathrm{M})$ for $1 \mathrm{hr}$ then stimulated with LPS (100ng/ml) for 18hr. ADA=Adenosine deaminase. Results are representative of three independent experiments from individual volunteers. 


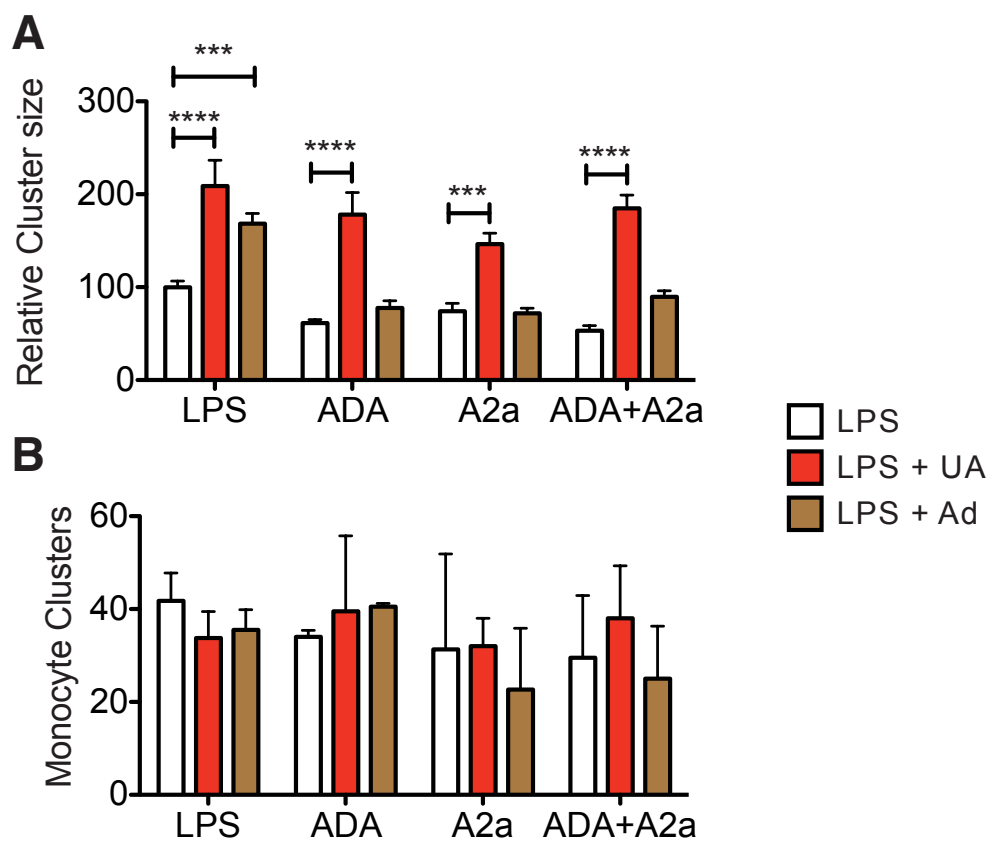

Figure 3.20 UA enhances LPS-induced aggregation independently of adenosine signalling.

Monocytes were treated as in figure 3.19. A: Monocyte cluster size relative to LPS alone. B: Average number of clusters in each field of view. ${ }^{* * *} p<0.001,{ }^{* * * *} p<0.0001$, Two-way ANOVA with Bonferroni post-test. Results are mean with SEM of A: total clusters per field of view and are representative of three independent experiments from individual volunteers and B: total number of clusters and are combined from three independent experiments from individual volunteers.

\subsubsection{Monocyte adhesion molecule expression}

Monocytes use adhesion molecules to move from the circulation into the tissues. CD162 and CD62L promote rolling and tethering to the endothelium ${ }^{190,191}$. CD11b is involved in firm adhesion and CD31 in transmigration ${ }^{192}$. I hypothesised that UA was increasing the expression of these adhesion molecules in LPS activated monocytes and thereby increasing aggregation.

To determine the effect of UA on the expression of monocyte adhesion molecules I measured CD62L, CD11b, CD31 and CD162 after treatment with combinations of UA, adenosine and LPS. UA and adenosine had no effect on adhesion molecule expression in untreated (figure 3.21A) or LPS treated monocytes (figure 3.21B). Therefore, it was unlikely that these adhesion molecules were contributing to the increased LPS-induced homotypic monocyte aggregation caused by UA. 
A Media
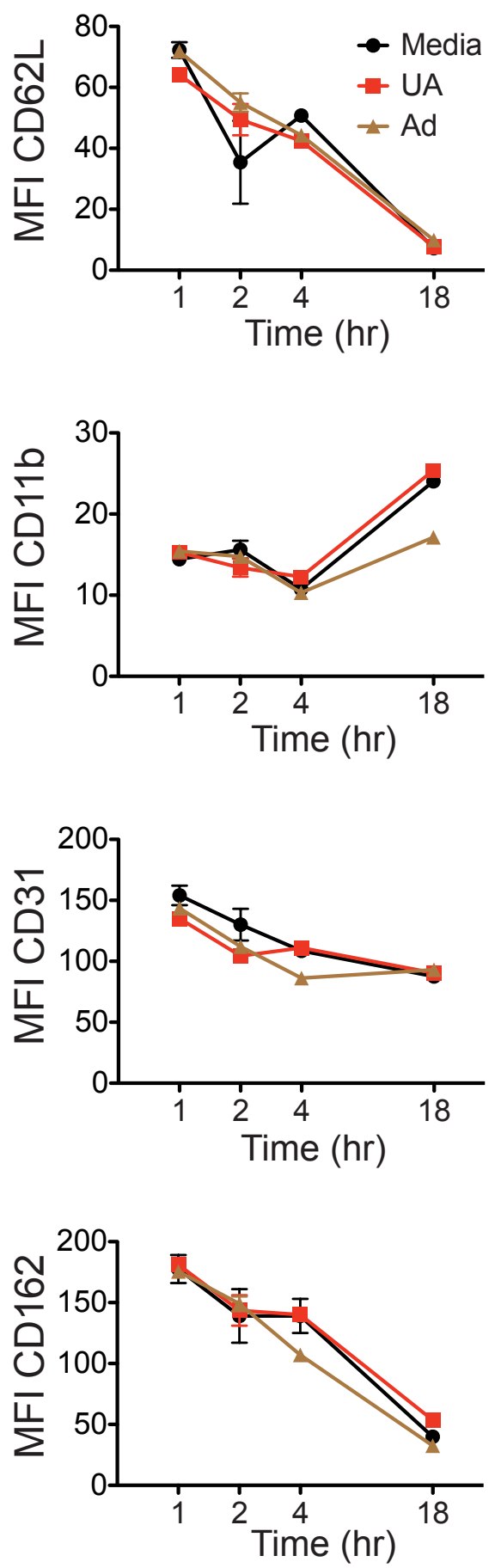

B LPS
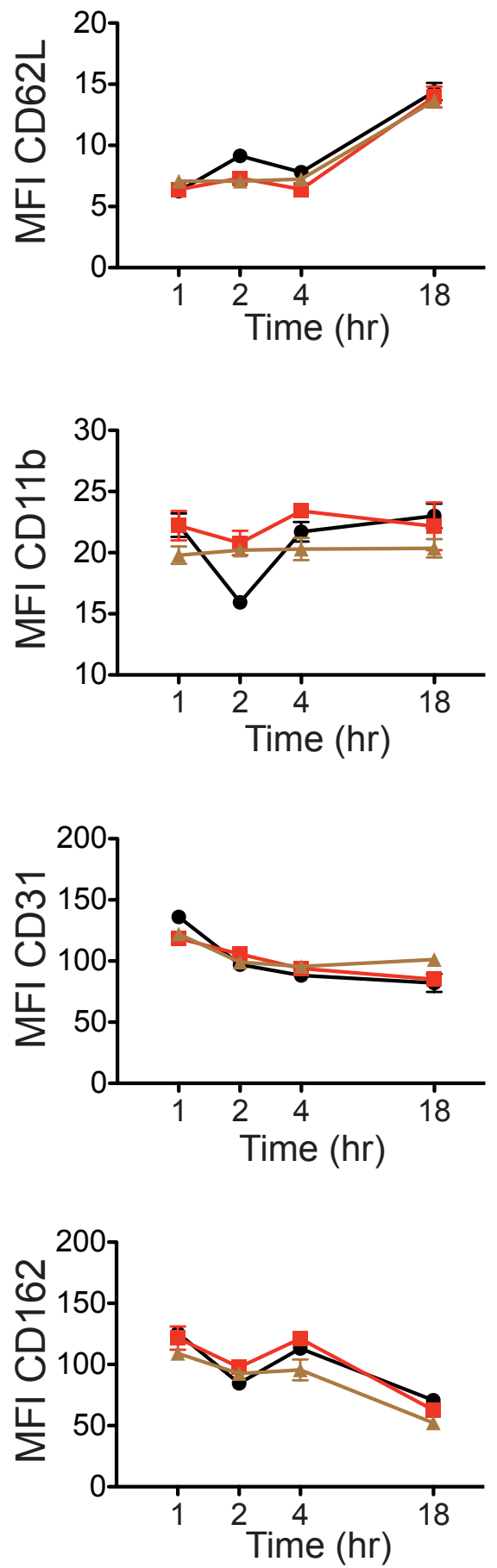

Figure 3.21 Monocyte adhesion molecule expression is unchanged by UA or adenosine.

A: Human blood monocytes were cultured in the absence (black lines) or presence of UA (0.6mM, brown lines) or adenosine $(0.1 \mathrm{mM}$, red lines) $B$ : As in (A) with the addition of LPS (100ng/ml). Surface marker expression was analysed at 1, 2, 4 and $18 \mathrm{hr}$ by flow cytometry. Results are mean with SEM of triplicate measurements and are representative of two independent experiments from individual volunteers. 


\subsubsection{Monocyte chemokine production}

Activated monocytes produce chemokines that recruit immune cells to areas of inflammation. It has been demonstrated that UA can directly stimulate nonimmune cells to produce $\mathrm{CCL} 2^{72}$ and the Harper group has shown that hyperuricaemia correlates with increased CCL2 levels and monocyte numbers in the blood ${ }^{128}$. CCL2, along with CCL3, CCL4 and CCL7 are monocyte chemoattractants, which are also produced by activated monocytes ${ }^{193,194}$. Therefore, I investigated UA's effect on monocyte chemokine production to see if it was linked to LPS-induced homotypic aggregation.

I measured monocyte release of CCL2, CCL3, CCL4 and CCL7 after exposure to different combinations of UA, adenosine and LPS. Unstimulated monocyte chemokine production was negligible and was not affected by either UA or adenosine treatment alone. In LPS treated monocytes there was a trend toward UA decreasing CCL3, CCL4 and CCL7 compared to LPS alone, which was not statistically significant. The addition of adenosine to LPStreated monocytes significantly decreased production of all four chemokines (figure 3.22). From these results it was evident that UA was not increasing LPS-induced monocyte homotypic aggregation by augmenting production of chemokines investigated in this study. 
A
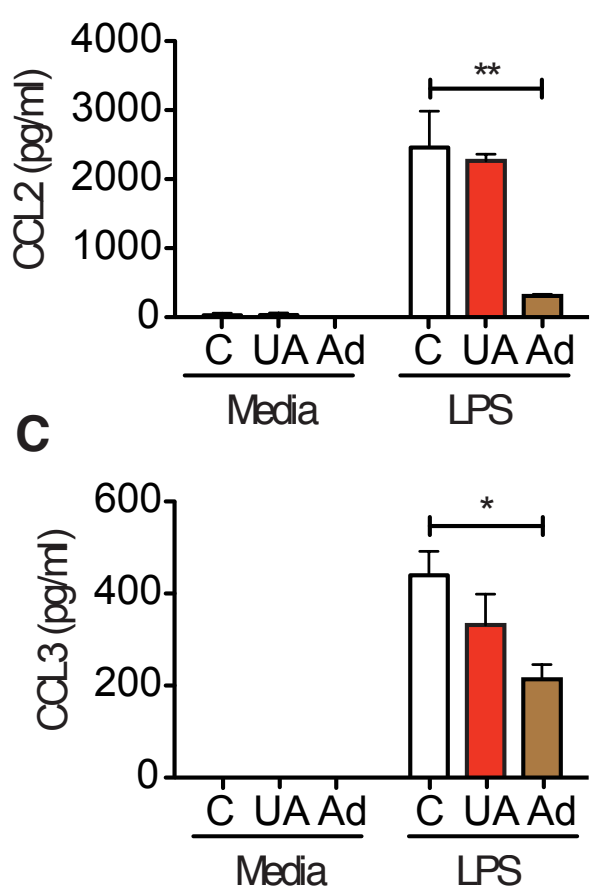

B
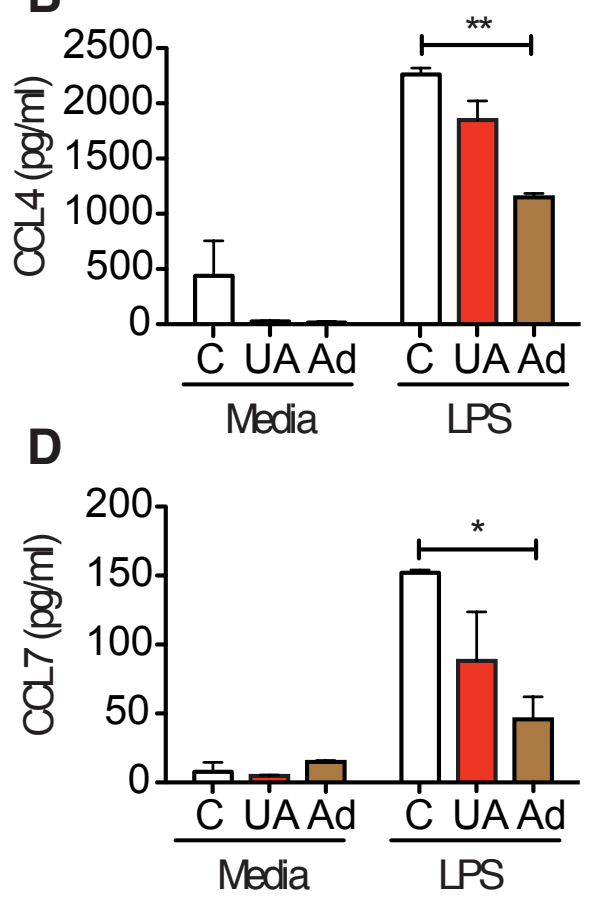

Figure 3.22 Monocyte chemokine production is not affected by UA.

Human blood monocytes were pre-treated with or without UA (0.6mM) or adenosine $(0.1 \mathrm{mM})$ for $1 \mathrm{hr}$ then stimulated with LPS $(100 \mathrm{ng} / \mathrm{ml})$ for up to $18 \mathrm{hr}$. Chemokine production was assessed by multiplex cytokine bead array at 4hr (CCL2), $1 \mathrm{hr}$ (CCL3), $2 \mathrm{hr}$ (CCL4) and 18hr (CCL7). ${ }^{*} p<0.05,{ }^{* *} p<0.01$. Two-way ANOVA with Bonferroni post-test. Results are mean with SEM of triplicate measurements and are representative of two independent experiments from individual volunteers. 


\subsection{Summary}

The purpose of this chapter was to explore the effect of soluble UA, using an in vitro model of hyperuricaemia, on monocyte inflammatory responses to LPS and investigate the mechanisms through which soluble UA could be acting. To this end I have found that soluble UA initiated a silent suppressive switch in human blood monocytes that caused a functional shift in the presence of an immune stimulus. LPS stimulation combined with soluble UA treatment decreased pro-inflammatory cytokine release, enhanced IL-10 production and increased monocyte homotypic aggregation, without inducing monocyte cell death. Analysis of monocyte adhesion molecule expression and chemokine production showed that UA was not enhancing LPS-induced monocyte homotypic aggregation via upregulation of the adhesion molecules CD62L, CD11b, CD31 and CD162 or production of the chemokines CCL2, CCL3, CCL4 and CCL7. Despite the structural similarity between UA and adenosine there was no evidence that soluble UA was signalling through adenosine receptors to increase IL-10 production or monocyte aggregation.

Importantly, treatment with soluble UA alone decreased monocyte ASC expression and increased DUSP10. This may be the initial event that shapes the downstream changes to MAP kinase and NFKB signalling following stimulation with LPS and results in a more suppressive inflammatory cytokine profile and increased homotypic aggregation.

The focus of Chapter 3 was on the direct effect of soluble UA on ex vivo human monocytes and the signalling pathways involved in soluble UAmediated suppression of the LPS response. The next chapter investigates how modulating serum UA levels in vivo alters systemic and monocytic responses to LPS and compares acute and chronic hyperuricaemia in this context. 


\title{
Chapter 4:
}

\section{Modulating Serum Uric Acid}

\author{
In Vivo
}





\section{Modulating serum uric acid in vivo}

\subsection{Introduction}

Chapter 3 showed that treatment of human blood monocytes in vitro with soluble UA resulted in a suppressive cytokine response to LPS and decreased inflammatory signalling. Modelling hyperuricaemia in vivo provides a better understanding of the immunological effects of soluble UA on LPSinduced inflammation in a more physiological context.

Rodent models of acute and chronic hyperuricaemia have been used mainly to investigate the effectiveness of potential urate-lowering compounds ${ }^{139,140,195}$ and several studies indicate that in vivo hyperuricaemia has a regulatory role during inflammation ${ }^{117,157,165}$. Combined with my in vitro results from Chapter 3 these studies provided a solid rationale for investigating the immunosuppressive effects of soluble UA in vivo.

Acute and chronic hyperuricaemia are associated with different pathologies. Acute hyperuricaemia during tumour lysis syndrome is a result of cell death induced by chemotherapy ${ }^{91}$. Chronic hyperuricaemia is commonly associated with metabolic syndrome and is the main risk factor for gout ${ }^{35}$. Modelling both acute and chronic hyperuricaemia will provide an insight into whether they have distinct effects during inflammation.

Additionally, hyperuricaemia can be treated with different methods of uratelowering therapy (ULT) such as allopurinol and rasburicase, which provides an opportunity to investigate how current ULT may be influencing systemic and monocyte immune responses to inflammation.

\subsection{Aim}

The aim of this chapter was to investigate how manipulation of serum UA levels alters the in vivo inflammatory response to LPS. 


\subsection{Results}

\subsubsection{A mouse model of acute hyperuricaemia}

To raise serum UA in C57 mice I used a combinatorial approach, administering the uricase inhibitor oxonic acid (OA) followed by a bolus injection of soluble UA. Combined OA and UA treatment raised mouse serum UA levels 2-fold at $1 \mathrm{hr}$ post-UA administration (Figure 4.1). This ratio was comparable to the differences observed in human populations, where healthy individuals have serum UA levels of $240-360 \mu \mathrm{M}$ while hyperuricaemics often have levels of $500-600 \mu M^{196}$.

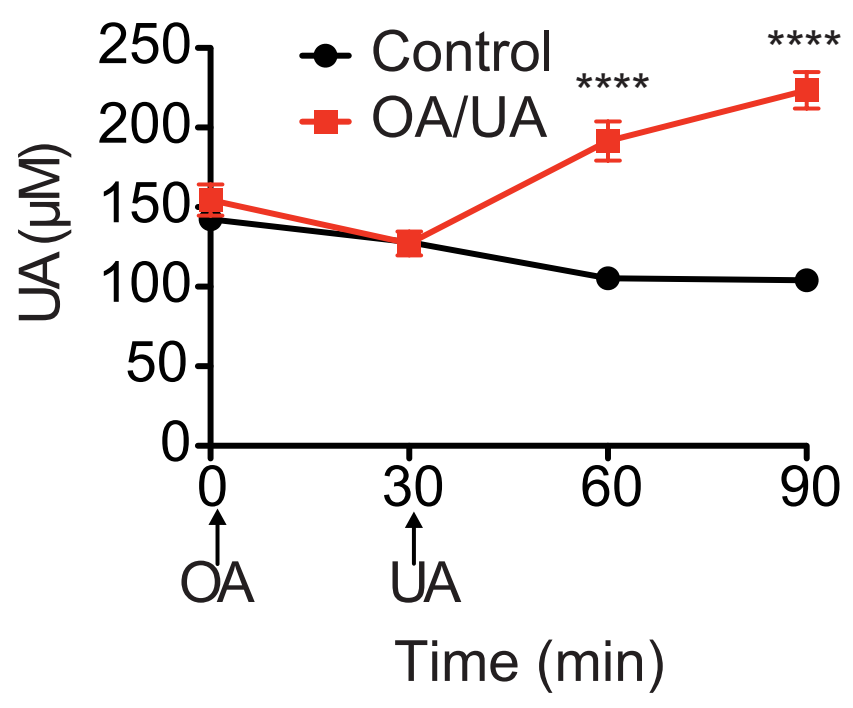

Figure 4.1 OA and UA induce acute hyperuricaemia in C57 mice.

Serum UA levels of C57 mice $(n=5)$ injected with oxonic acid i.p. (OA 300mg/kg) and soluble UA i.v. (12ug/kg) at the times indicated. Serum UA was measured using a fluorometric assay. ${ }^{* * * *} p<0.0001$, two-way ANOVA with Bonferroni post-test. Results are mean with SEM of triplicate measurements and are representative of two independent experiments. 
Next I injected OA/UA treated mice with LPS i.v. and measured serum IL-10 over a $24 \mathrm{hr}$ period. IL-10 peaked at $1 \mathrm{hr}$ post-LPS treatment and was enhanced by combined OA/UA treatment (figure 4.2). These data confirmed that hyperuricaemia increased systemic IL-10 production in vivo in response to LPS.

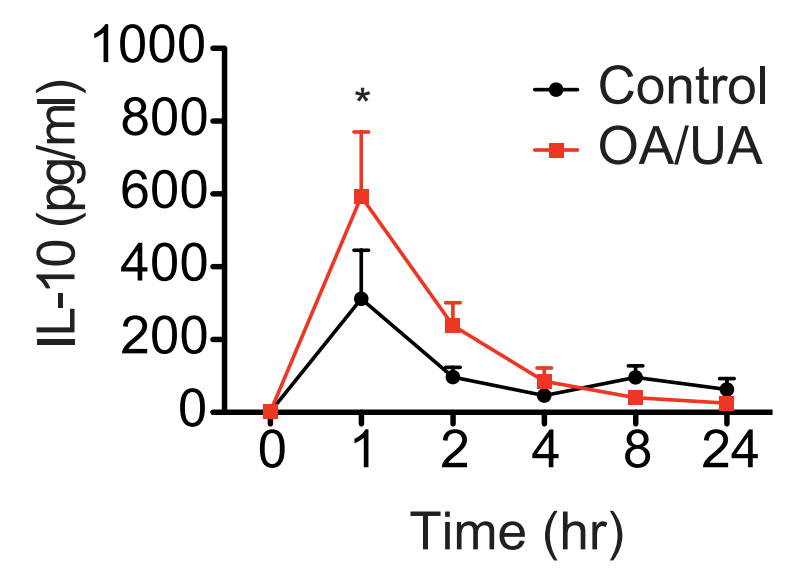

Figure $4.2 \quad$ IL-10 peaks in mouse serum 1 hour after LPS challenge.

Serum IL-10 levels of C57 mice $(n=4)$ pre-treated with or without oxonic acid (OA $300 \mathrm{mg} / \mathrm{kg}$, 30min) and UA (12ug/kg, $1 \mathrm{hr})$, then administered LPS i.v. (40 $\mathrm{ng} / \mathrm{kg})$. Serum IL-10 was measured by ELISA. ${ }^{*} p<0.05$, two-way ANOVA with Bonferroni post-test. Results are mean with SEM of triplicate measurements and are representative of two independent experiments.

\subsubsection{Profiling the systemic cytokine response to LPS during acute hyperuricaemia}

In Chapter 3 I showed that in vitro treatment with soluble UA suppressed the proinflammatory cytokine response of monocytes stimulated with LPS. Therefore, I measured the levels of proinflammatory serum cytokines in naïve and hyperuricaemic mice at the peak of the IL-10 response, $1 \mathrm{hr}$ after LPS administration.

Hyperuricaemia had no effect on serum cytokine levels in the absence of LPS stimulation. Serum IL-10 was increased in hyperuricaemic mice compared to controls following LPS administration (figure 4.3A). TNF $\alpha$ and IL-12p40 were reduced in hyperuricaemic mice after LPS administration (figure 4.3B and D). There was no difference in serum IL-6 levels in hyperuricaemic versus control 
mice following LPS (figure 4.3E). Serum IL-1 $\beta$ levels were below the detection limit of the ELISA in all of the conditions investigated (figure 4.3C). These results indicated that hyperuricaemia was suppressing the inflammatory response to LPS in vivo.
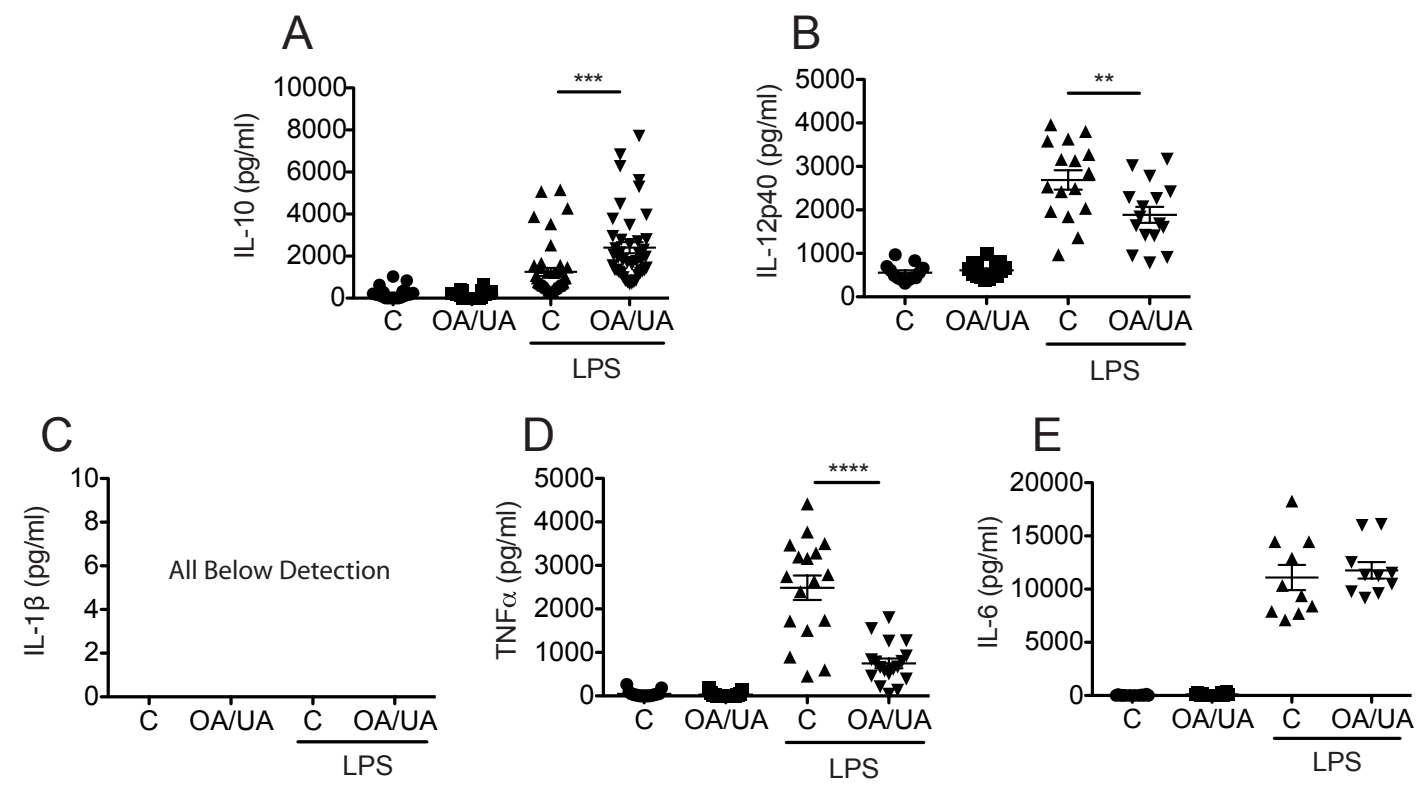

Figure 4.3 In vivo hyperuricaemia reduces inflammatory cytokines and increases IL-10 in response to LPS.

Serum cytokine levels of C57 mice pre-treated with or without oxonic acid (OA $300 \mathrm{mg} / \mathrm{kg}, 30 \mathrm{~min})$ and UA (12ug/kg, $1 \mathrm{hr})$, then administered LPS i.v. (40ng/kg, $1 \mathrm{hr})$. A: Serum IL-10 (n=43), B: IL-12p40 ( $n=16), C: I L-1 \beta(n=10), D: T N F \alpha(n=18)$ and $E$ : IL-6 ( $n=10)$ were measured by ELISA. ${ }^{*} p<0.05,{ }^{* *} p<0.01,{ }^{* * *} p<0.001,{ }^{* * * *} p<0.0001$, student's t-test. Results are mean with SEM and are combined from at least two independent experiments.

\subsubsection{The in vivo cellular response to acute hyperuricaemia and LPS}

Next I investigated whether soluble UA caused the same signalling changes I had observed in human monocytes treated with soluble UA in vitro, namely decreased MAP kinase phosphorylation and ASC expression, and differential NFкB subunit nuclear translocation (appendices 7.2 and 7.1 and figure 3.9). Before I could look at signalling I needed to identify which cell types might be increasing IL-10 production in response to LPS during hyperuricaemia. To do this I isolated spleens from control and hyperuricaemic mice after LPS administration, cultured the whole splenocyte preparations with GolgiStop, an 
intracellular protein transport inhibitor containing monensin, to prevent cytokine secretion then stained for cell surface markers and intracellular IL-10. There was a population of IL-10 positive cells in the spleen that were predominantly CD11b+ and Ly6C+, indicating that splenic monocytes were a source of IL-10 (figure 4.4A). By gating first on CD11b+/Ly6C+ cells (9\% of whole splenocyte preparations) I saw that there was an increase in IL-10+ monocytes in hyperuricaemic versus control mice after LPS treatment (figure 4.4B). These results indicated that the CD11b+/Ly6C+ positive monocytes in the spleen were producing $\mathrm{IL}-10$ and were responding to acute hyperuricaemia by increasing IL-10 production following LPS challenge. I also investigated intracellular production of $\mathrm{IL}-12 \mathrm{p} 40$ and $\mathrm{TNF} \alpha$ but could not detect either cytokine in splenic monocyte preparations.

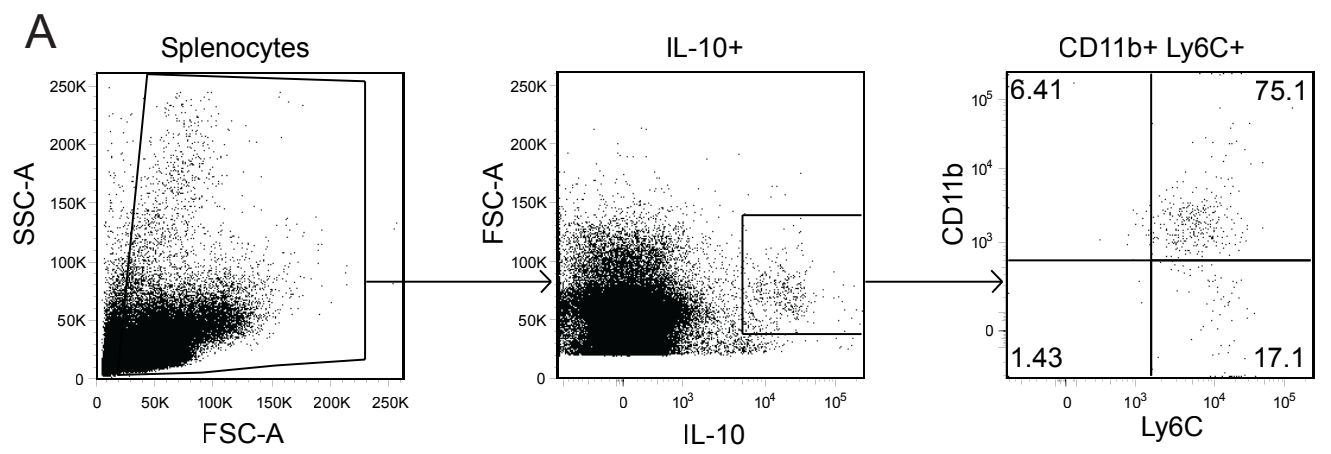

B
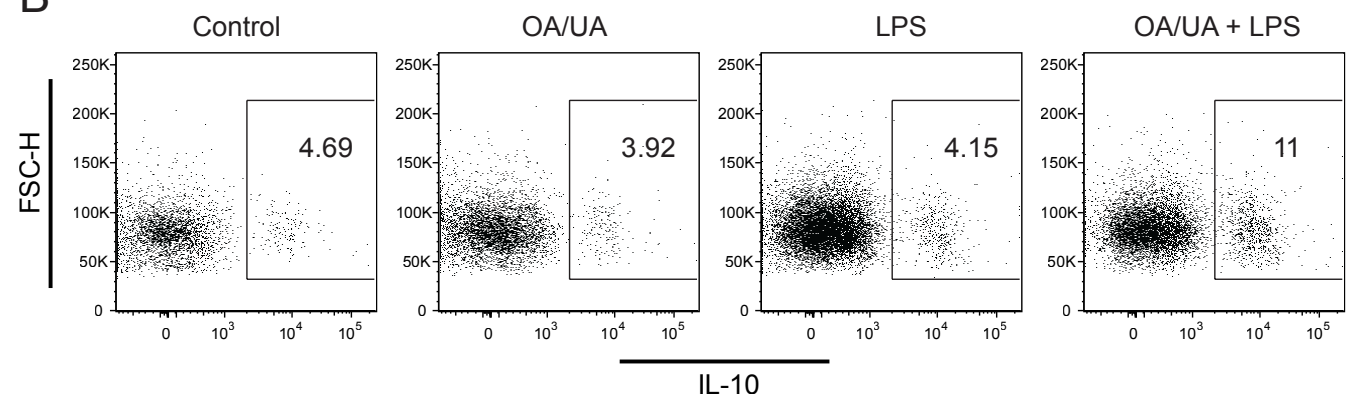

Figure 4.4 A population of splenic CD11b+/Ly6C+ monocytes produces IL10 upon LPS challenge and increases in frequency in hyperuricaemic mice. Splenic cells from $C 57$ mice $(n=5)$ pre-treated with or without oxonic acid (OA $300 \mathrm{mg} / \mathrm{kg}, 30 \mathrm{~min})$ and UA (12ug/kg, $1 \mathrm{hr}$ ), then administered LPS i.v. (40 $\mathrm{ng} / \mathrm{kg}, 1 \mathrm{hr}$ ). Cells were incubated in GolgiStop (1:1000, 4hr) then stained with cell surface and intracellular fluorescent antibodies and analysed by flow cytometry. A: Gating strategy to identify IL-10+ monocytes, B: Percent IL-10+ monocytes in each treatment group. Results are representative of two independent experiments. 
Next I investigated the effects of hyperuricaemia and systemic LPS administration on splenic monocyte inflammatory signalling pathways. Following induction of acute hyperuricaemia and systemic inflammation with LPS I purified splenic monocytes using density gradient centrifugation followed by negative magnetic selection then isolated cytosolic and nuclear protein for western blotting.

In vivo hyperuricaemia alone reduced splenic monocyte ASC expression (figure 4.5A). Following LPS stimulation DUSP10 expression was increased and phosphorylation of $\mathrm{p} 38$ and ERK1/2 MAP kinases was decreased in monocytes from hyperuricaemic mice compared to control (figure 4.5A). I $\mathrm{kB} \alpha$ expression was also increased in the cytosol of splenic monocytes from hyperuricaemic mice challenged with LPS (figure 4.5A), which coincided with a decrease in NFkB p65 nuclear translocation (figure 4.5B). Densitometric analysis of the depicted western blots is presented in appendix 7.3A-G.

These results showed that inflammatory MAP kinase and NFKB signalling within splenic monocytes was inhibited by acute hyperuricaemia during in vivo immune challenge with LPS. 


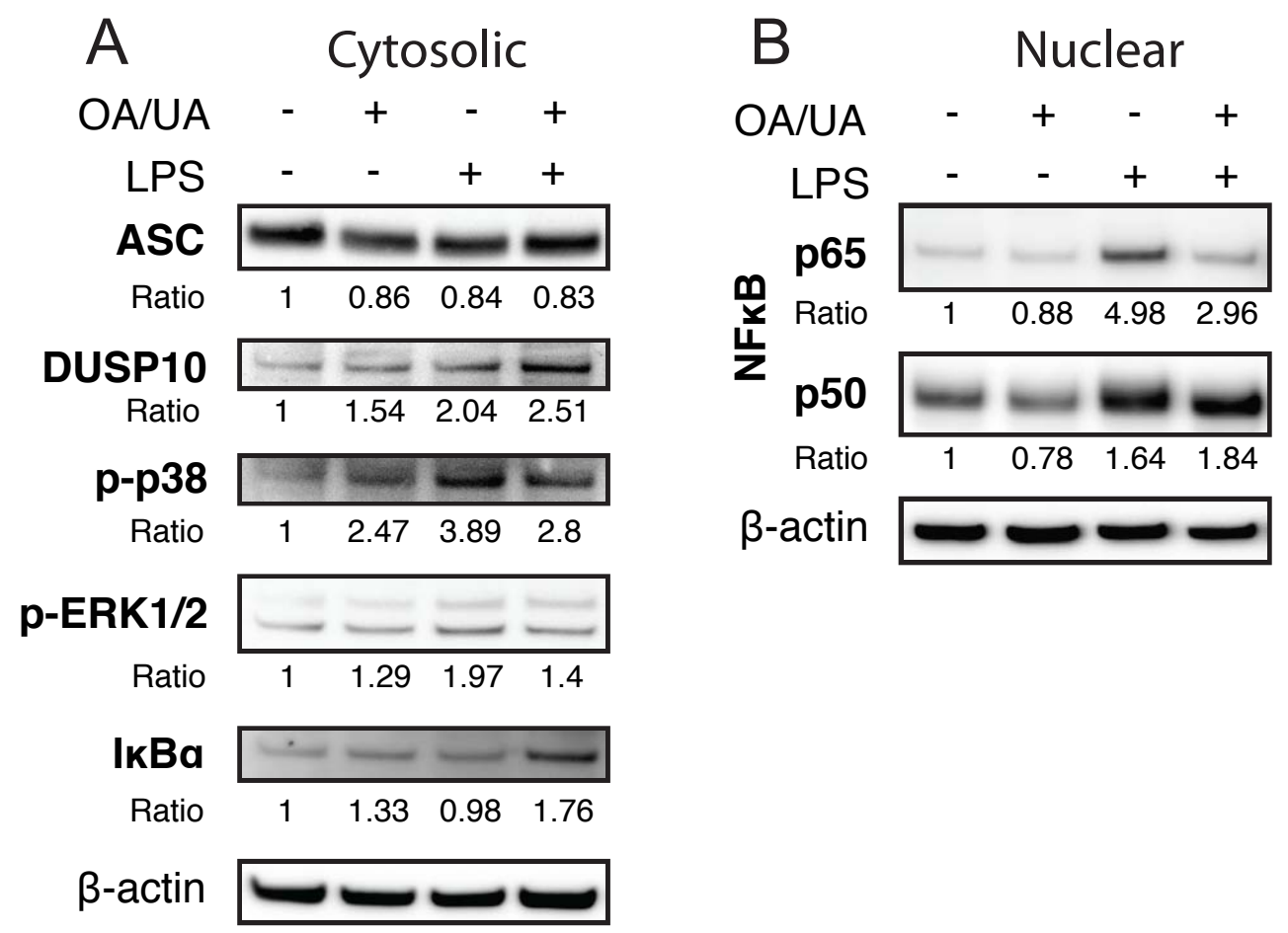

Figure 4.5 Hyperuricaemia reduces ASC expression and MAP kinase phosphorylation in splenic monocytes and decreases NFKB p65 nuclear translocation.

A: Cytosolic and B: nuclear protein from purified splenic monocytes isolated from C57 mice ( $n=5)$ pre-treated with or without oxonic acid (OA $300 \mathrm{mg} / \mathrm{kg}, 30 \mathrm{~min})$ and UA (12ug/kg, 1hr), then administered LPS i.v. (40ng/kg, $1 \mathrm{hr})$. Protein was analysed by western blotting. Results are representative of two independent experiments.

ASC is a central component of the NLRP3 inflammasome, which is responsible for the cleavage and maturation of pro-IL-1 $\beta$ into active IL-1 $\beta^{115}$. Therefore, I investigated the expression levels of other inflammasome components in splenic monocytes from hyperuricaemic mice.

Acute hyperuricaemia increased pro-caspase1 expression in both unstimulated and LPS-stimulated monocytes, but this appeared to be restricted to the larger isoform of pro-capase1 (upper band). (figure 4.6). There was also a decrease in active IL-1 $\beta$ in LPS treated monocytes from hyperuricaemic mice (figure 4.6). Densitometric analysis of the depicted western blots is presented in appendix 7.3H-L. 


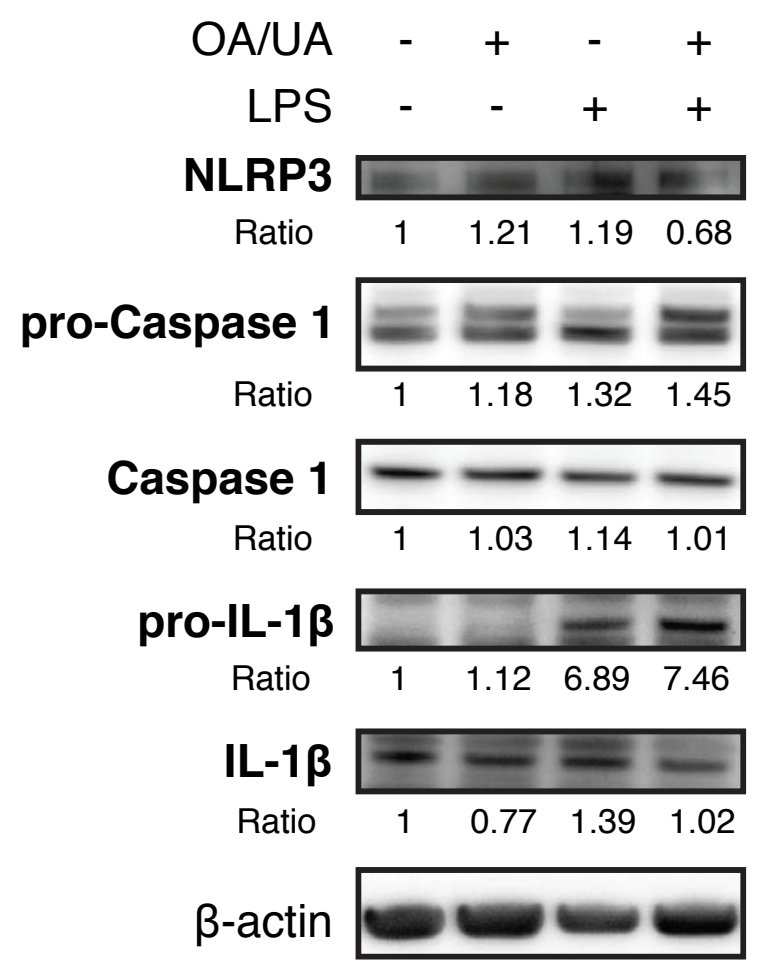

Figure 4.6 Hyperuricaemia increases pro-caspase1 expression and decreases active IL-1 $\beta$ in splenic monocytes.

Cytosolic protein from purified splenic monocytes isolated from C57 mice $(n=5)$ pretreated with or without oxonic acid (OA 300 mg/kg, 30min) and UA (12ug/kg, 1hr), then administered LPS i.v. (40ng/kg, $1 \mathrm{hr})$. Protein was analysed by western blotting. Results are representative of two independent experiments.

\subsubsection{Lowering serum UA in vivo}

The results from the previous sections showed that acute in vivo hyperuricaemia reduced systemic inflammatory cytokines in response to LPS, increased serum IL-10 and inhibited monocyte inflammatory signalling pathways. Next I tested the hypothesis that lowering serum UA would augment the systemic and cellular inflammatory response to LPS.

\section{Allopurinol}

To investigate the effect of lowering UA in vivo I treated mice with the ULT allopurinol for $3 \mathrm{hr}$, which significantly reduced serum UA levels (figure 4.7A). I then administered LPS i.v. and measured the levels of serum cytokines. Allopurinol treatment alone had no effect on serum cytokine levels (figure 4.7B-D). LPS-induced IL-10 production was decreased and IL-12p40 was 
increased in allopurinol treated mice versus control (figure 4.7B-C). Allopurinol treatment had no effect on serum TNF $\alpha$ levels and there was a trend towards increased IL-6 in LPS treated mice (figure 4.7dD-E).
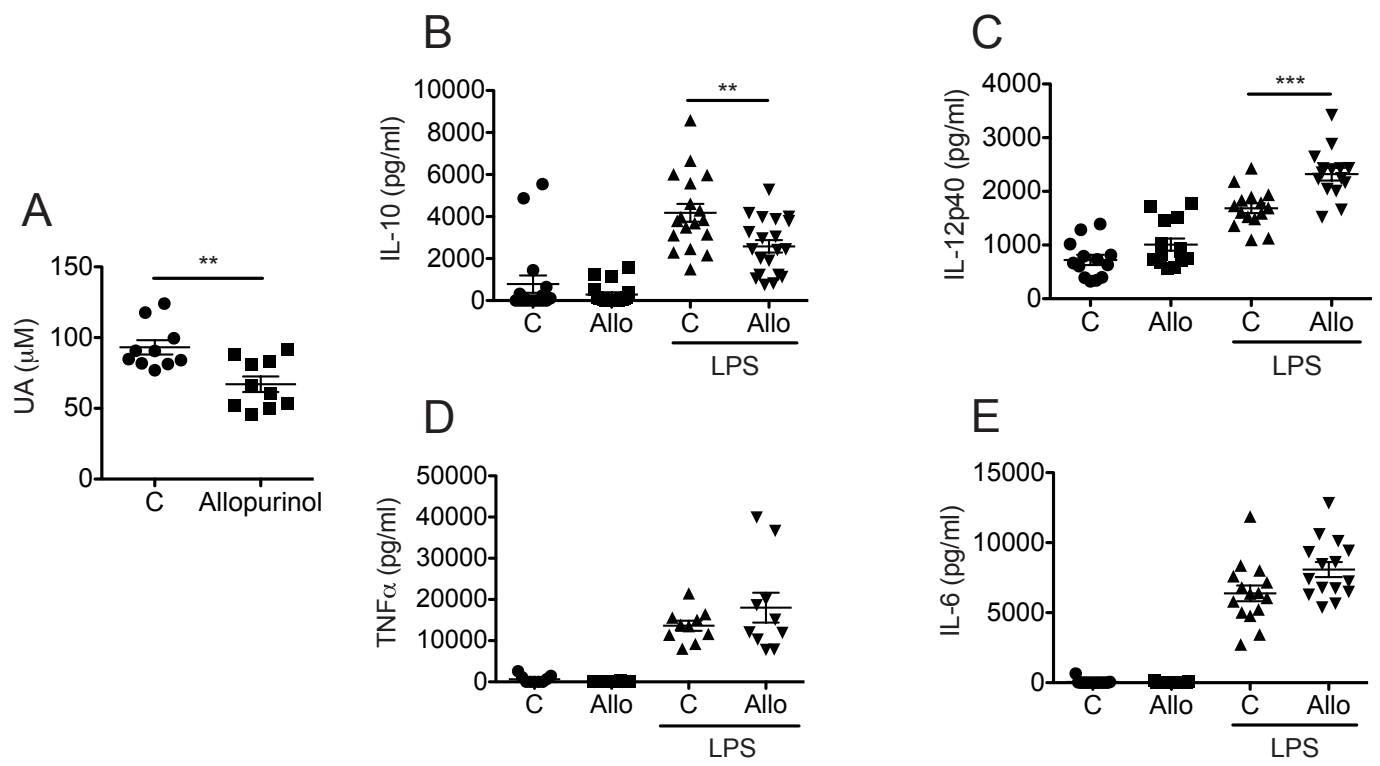

Figure 4.7 Allopurinol treatment lowers serum UA and increases LPSinduced systemic inflammation.

A: Serum UA and B-E: cytokine levels of C57 mice pre-treated with or without allopurinol (30mg/kg, 3hr) then administered LPS i.v. (40ng/kg, $1 \mathrm{hr})$. Serum UA was measured using a fluorometric assay. Serum cytokines were measured by ELISA. ${ }^{* *} p<0.01,{ }^{* * *} p<0.001$, student's $t$-test. Results are mean with SEM and are combined from at least two independent experiments. $A: n=10, B: n=20, C: n=15, D$ $n=10, E: n=15$. 
I next investigated the effect of allopurinol and LPS treatment on protein expression in murine splenic monocytes by western blotting.

Allopurinol had no effect on monocyte ASC or DUSP10 expression (figure 4.8A). There was a decrease in LPS-induced p38 MAP kinase phosphorylation in allopurinol treated mice versus LPS alone but no changes to phosphorylated ERK1/2 (figure 4.8A). Allopurinol treatment resulted in a slight decrease in cytosolic $1 \kappa \mathrm{B} \alpha$ expression (figure 4.8A, lower band) and a corresponding increase in NFKB p65 and p50 nuclear translocation in monocytes from LPS treated mice (figure 4.8B). Densitometric analysis of the depicted western blots is presented in appendix 7.4A-G.

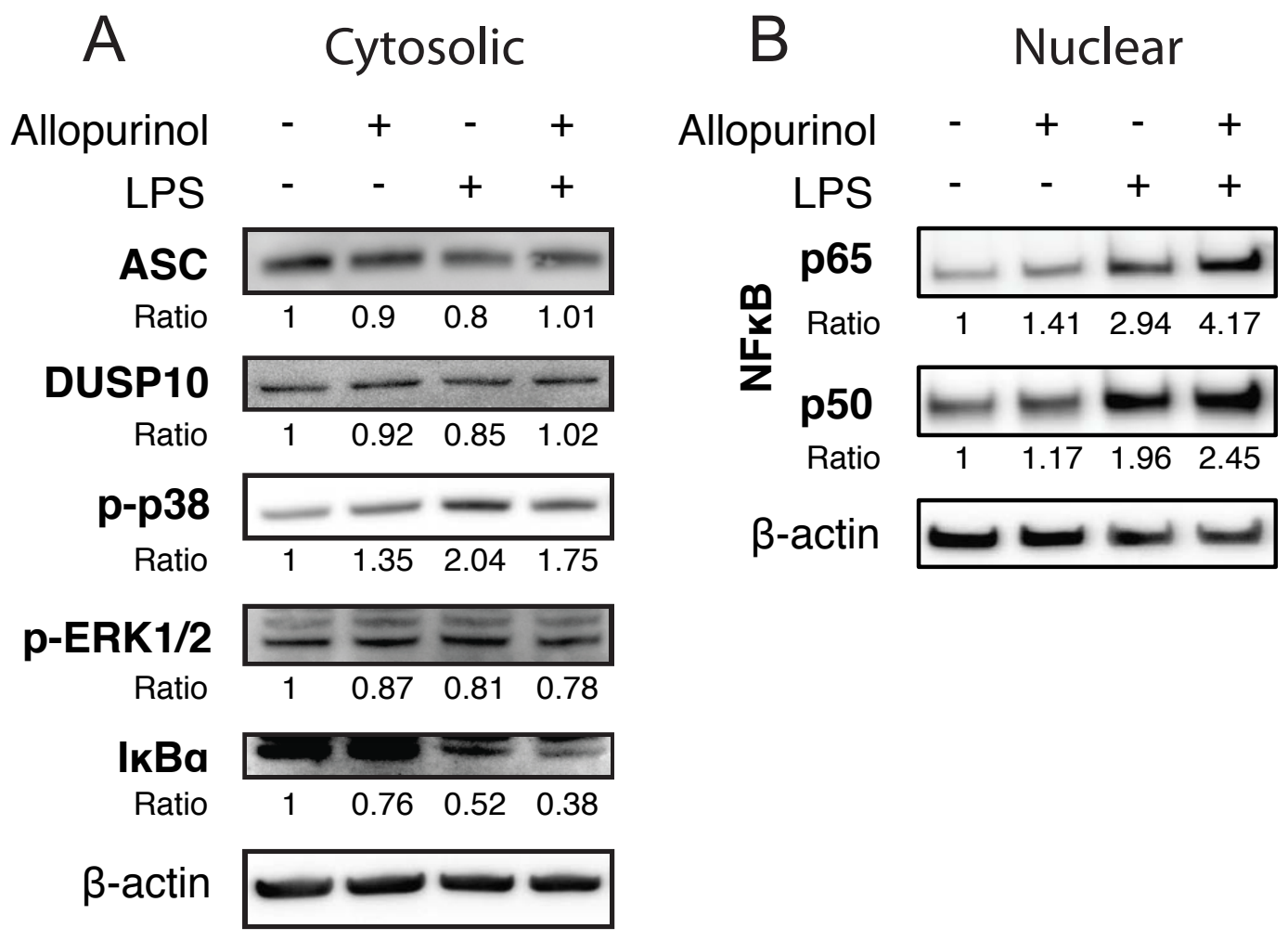

Figure 4.8 Allopurinol treatment reduces monocyte $I K B \alpha$ expression and increases NFKB p65 nuclear translocation.

A: Cytosolic and B: nuclear protein from purified splenic monocytes isolated from C57 mice $(n=5)$ pre-treated with or without allopurinol $(30 \mathrm{mg} / \mathrm{kg}$, 3hr) then administered LPS i.v. (40ng/kg, 1hr). Protein was analysed by western blotting. Results are representative of two independent experiments. 


\section{Rasburicase}

I treated mice with rasburicase and found that, like allopurinol, a single rasburicase dose was sufficient to reduce serum UA after $3 \mathrm{hr}$ (figure 4.9A). I then measured cytokine levels in serum from LPS treated and control mice. There was no effect of rasburicase alone on serum cytokines (figure 4.9B-E). Although not statistically significant, rasburicase treatment produced a trend towards decreased IL-10 and increased IL-12p40 and IL-6 levels in LPS treated mice (figure 4.9B, C, E).
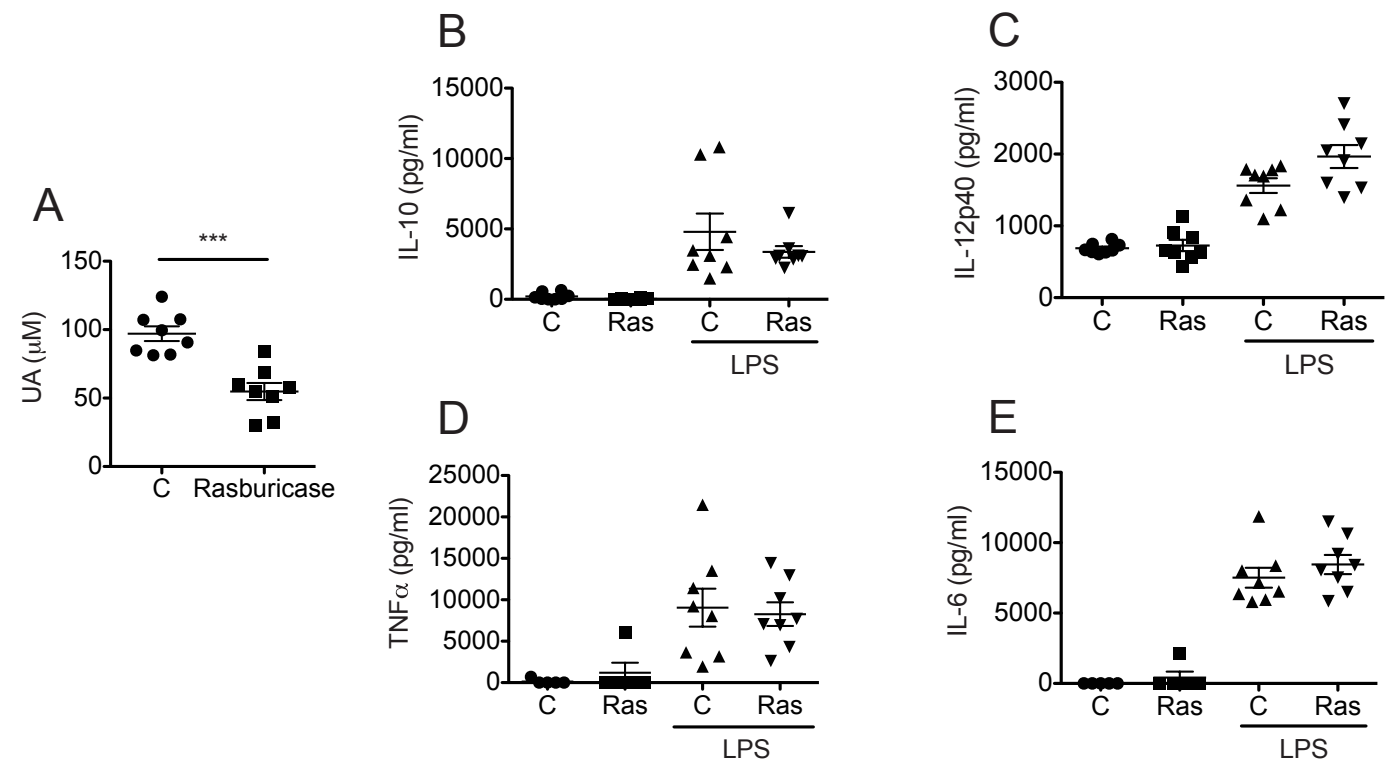

Figure 4.9 Rasburicase treatment lowers serum UA but does not alter LPSinduced systemic inflammation.

$A$ : Serum UA and B-E: cytokine levels of C57 mice pre-treated with or without rasburicase $(0.8 \mathrm{mg} / \mathrm{kg}$, 3hr) then administered LPS i.v. (40ng/kg, $1 \mathrm{hr})$. Serum UA was measured using a fluorometric assay. Serum cytokines were measured by ELISA. ${ }^{* * *} p<0.001$, student's t-test. Results are mean with SEM and are combined from at least two independent experiments. $A: n=8, B: n=8, C: n=8, D n=8, E: n=8$. 
Next I examined whether splenic monocyte signalling pathways were being affected by rasburicase treatment. I purified splenic monocytes from control, rasburicase, LPS and rasburicase plus LPS treated mice to measure cytosolic and nuclear protein expression by western blotting.

Rasburicase treatment alone increased monocyte ASC and DUSP10 expression, while LPS in combination with rasburicase reduced ASC and DUSP10 expression compared to LPS alone (figure 4.10A). Rasburicase had no effect on MAP kinase phosphorylation or $1 \kappa B \alpha$ expression (figure 4.10A). Following densitometric analysis the nuclear fractions showed a relative increase in NFKB p65 or p50 subunit translocation after LPS and rasburicase treatment (figure 4.11B). Densitometric analysis of the depicted western blots is presented in appendix 7.5A-G.

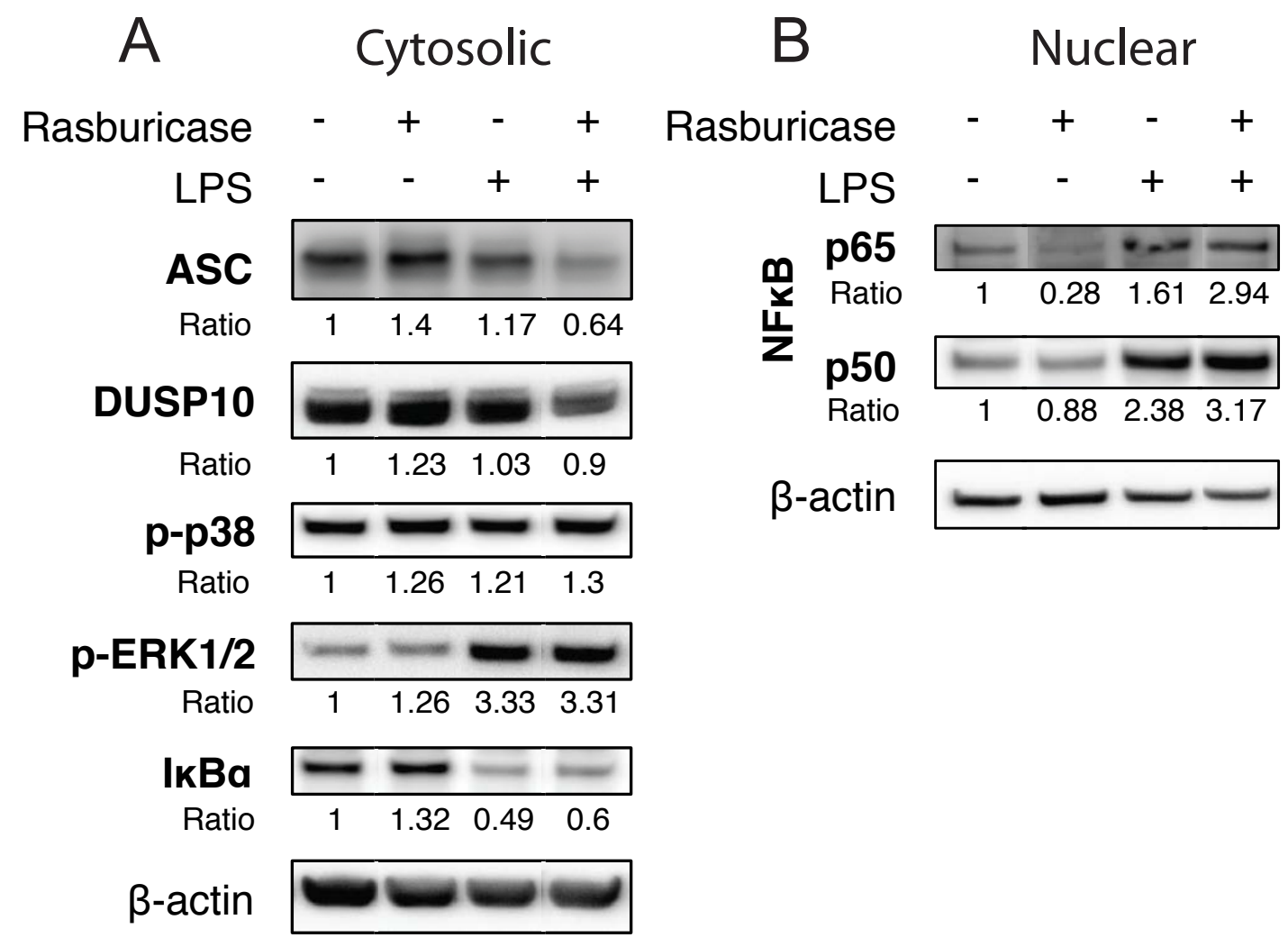

Figure 4.10 Rasburicase treatment alone increases monocyte ASC expression and NFKB nuclear translocation.

A: Cytosolic and B: nuclear protein from purified splenic monocytes isolated from C57 mice ( $n=5)$ pre-treated with or without rasburicase $(0.8 \mathrm{mg} / \mathrm{kg}$, 3hr) then administered LPS i.v. (40ng/kg, 1hr). Protein was analysed by western blotting. Results are representative of two independent experiments. Lanes have been cropped from a single blot for display purposes. 
I also compared the effects of allopurinol and rasburicase treatment on the expression of NLRP3 inflammasome components in splenic monocytes from mice with or without LPS administration. Both allopurinol and rasburicase treatment alone decreased monocyte expression of pro-caspase 1 and this decrease was still evident following LPS challenge. Rasburicase also decreased pro-IL-1 $\beta$ after LPS (figure 4.11). Densitometric analysis of the depicted western blots is presented in appendix 7.6A-E.

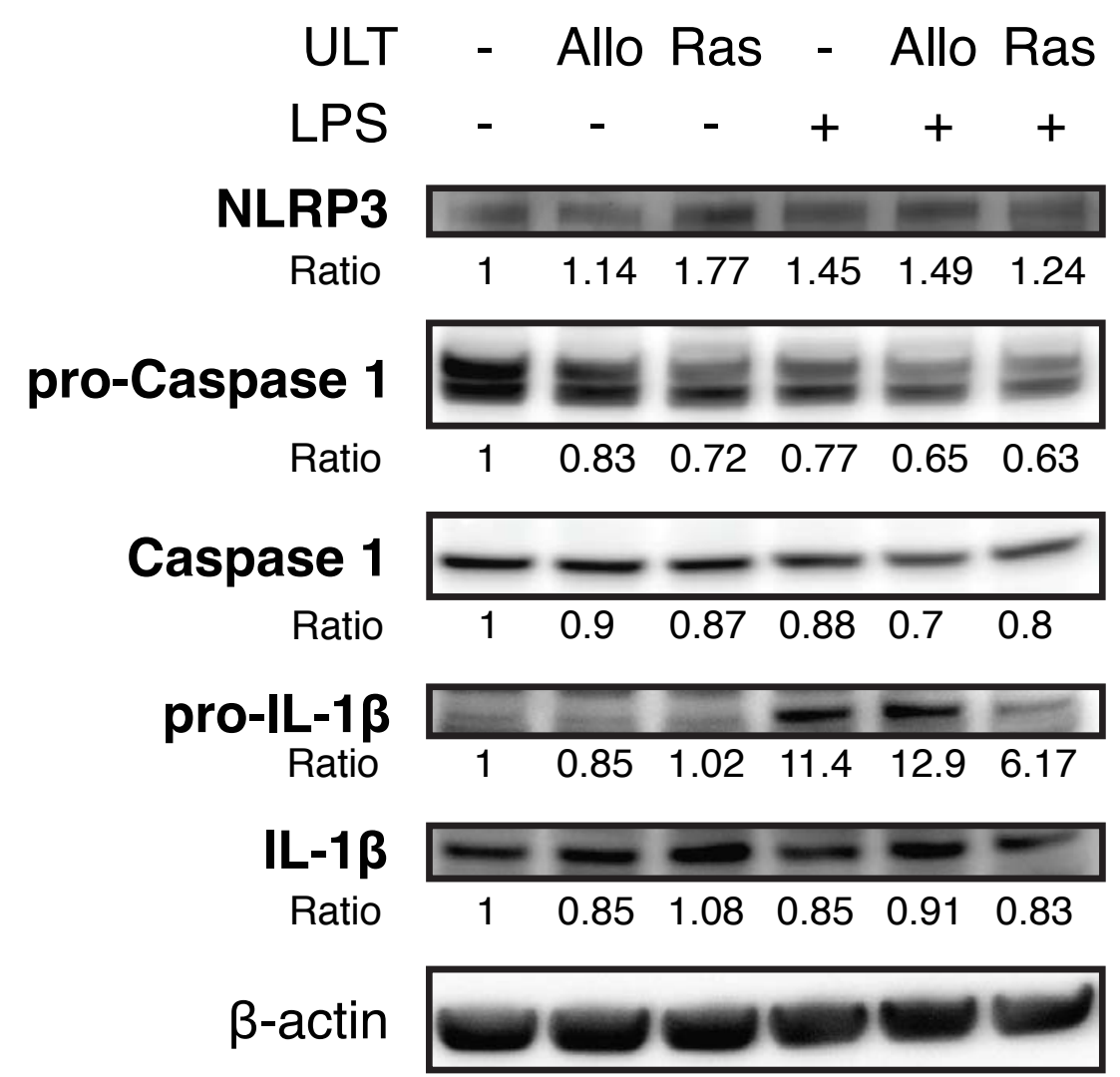

Figure 4.11 ULT with rasburicase increases NLRP3 but decreases procaspase1 and pro-IL-1 $\beta$ expression in splenic monocytes.

Cytosolic protein from purified splenic monocytes isolated from C57 mice $(n=5)$ pretreated with or without allopurinol $(30 \mathrm{mg} / \mathrm{kg}$, 3hr) or rasburicase $(0.8 \mathrm{mg} / \mathrm{kg}$, 3hr) then administered LPS i.v. (40ng/kg, $1 \mathrm{hr})$. Protein was analysed by western blotting. Results are representative of two independent experiments. 
Together these results showed that lowering serum UA with allopurinol but not rasburicase increased the systemic inflammatory cytokine response to LPS and decreased serum IL-10 levels. Allopurinol and rasburicase treatment also had differential effects on the inflammatory signalling pathways of splenic monocytes: allopurinol and rasburicase altered NFKB while only rasburicase altered ASC, NLRP3 and pro-IL-1 $\beta$ expression.

\subsubsection{A mouse model of chronic hyperuricaemia}

I had shown that acute changes to serum UA levels altered systemic cytokine responses and splenic monocyte inflammatory signalling in response to LPS. Next I wanted to investigate how chronic hyperuricaemia, which is closer to the human condition, affected systemic inflammation.

The Urah ${ }^{\text {PLT2/PLT2 }}$ (PLT2) mouse strain was identified during a mutagenesis screen and has a point mutation in the Urah gene that codes for 5hydroxyisourate (HIU) hydrolase ${ }^{90}$. Normally the endpoint of purine metabolism in mice is allantoin but the PLT2 strain has had its purine metabolic pathway disrupted at HIU hydrolase, the enzyme following uricase (figure 4.12). The effect of this mutation on the PLT2 strain's serum UA level has not been reported. I hypothesised that the mutation of HIU hydrolase and resulting build up of HIU would produce an increase in serum UA, possibly as a result of product inhibition of uricase by HIU. 


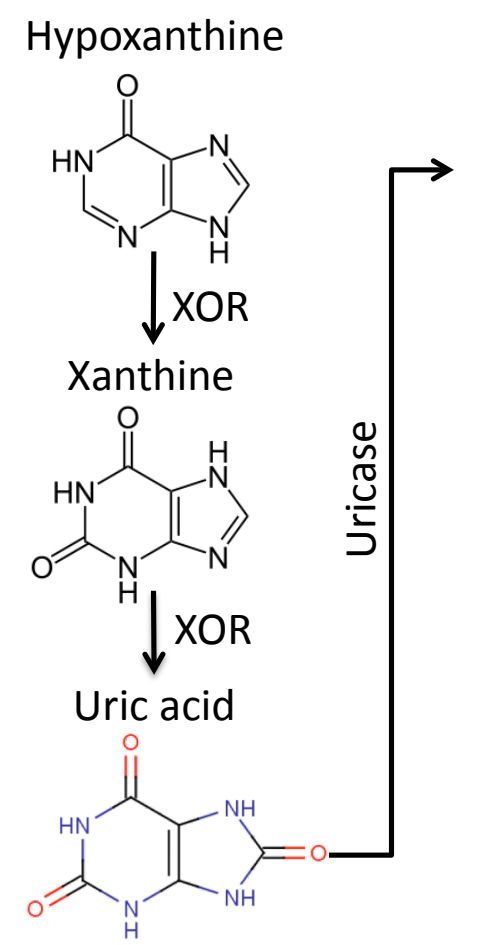

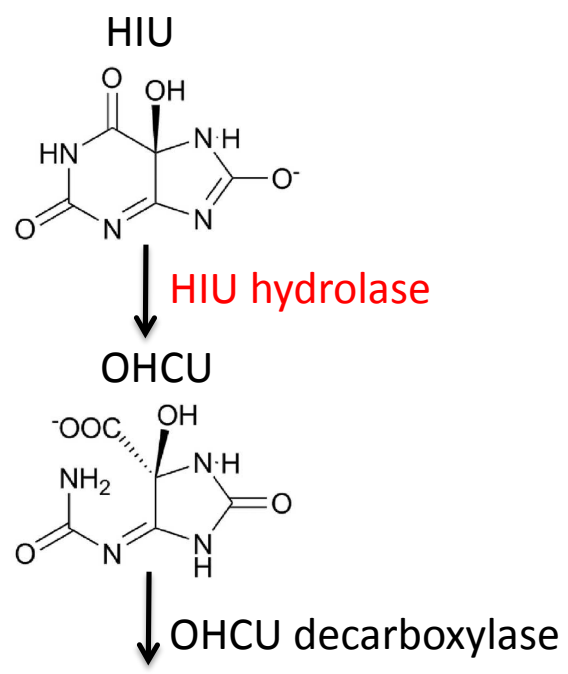<smiles>NC(=O)N[C@@H]1NC(=O)NC1=O</smiles>

Figure 4.12 Mouse purine metabolic pathway from hypoxanthine to allantoin. HIU hydrolase has been knocked out in the PLT2 mouse strain. XOR = xanthine oxidoreductase, HIU = 5-hydroxyisourate, $\mathrm{OHCU}=$ 2-oxo-4-hydroxy-4-carboxy-5ureidoimidazoline.

I measured the serum UA levels of PLT2 and C57 mice and found that PLT2 mice were hyperuricaemic (figure 4.13A). The 2-fold increase in serum UA between C57 and PLT2 mice was comparable to the acute hyperuricaemia I had generated in C57 mice using administration of OA and UA (figure 4.1).

Having confirmed that the PLT2 strain was indeed hyperuricaemic I investigated the effect of this chronic hyperuricaemia on serum cytokine levels using my LPS model of systemic inflammation.

There was no difference in cytokine levels of naïve C57 and PLT2 mice. Following LPS administration PLT2 mice had higher levels of the serum cytokines IL-10, IL12-p40 and TNF $\alpha$ and there was a trend towards higher IL6 (figure 4.13B-E). This differed from the effects of acute hyperuricaemia, which increased serum IL-10 but decreased proinflammatory cytokines in response to LPS. 

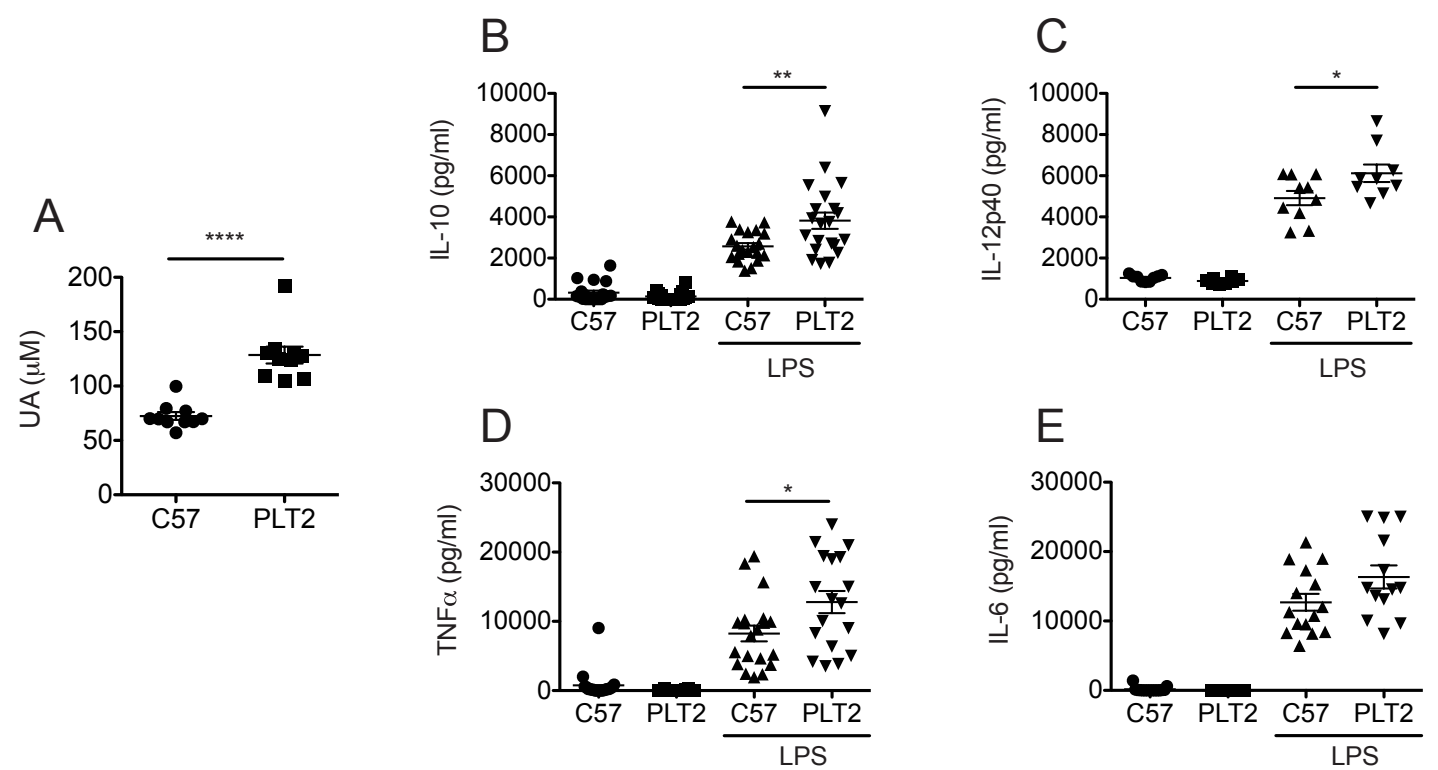

Figure 4.13 Serum UA and cytokine levels are higher in LPS treated PLT2 mice compared with $C 57$ mice.

A: Serum UA and B-E: cytokine levels of C57 and PLT2 mice administered LPS i.v. (40ng/kg, $1 \mathrm{hr})$. Serum UA was measured using a fluorometric assay. Serum cytokines were measured by ELISA. ${ }^{*} p<0.05$, ${ }^{* *} p<0.001,{ }^{* * * *} p<0.0001$, student's $t$ test. Results are mean with SEM and are combined from at least two independent experiments. $A: n=10, B: n=20, C: n=9, D n=18, E: n=13$.

I also compared the effect of chronic hyperuricaemia on inflammatory signalling molecules in splenic monocytes from the C57 and PLT2 mice. I isolated splenic monocytes from the spleens of naïve and LPS treated C57 and PLT2 mice and prepared cytosolic and nuclear protein for western blotting. There were no clear differences in either monocyte ASC expression or MAP kinase phosphorylation between C57 and PLT2 mice (figure 4.14A). DUSP10 expression was higher and $I_{\kappa} B \alpha$ levels were the same in the PLT2 mouse following LPS stimulation (figure 4.14A) and there was little difference in NFKB p65 and p50 subunit translocation into the nucleus of monocytes from PLT2 compared to C57 mice with or without in vivo LPS stimulation (figure 4.14B). Densitometric analysis of the depicted western blots is presented in appendix 7.6A-G. 

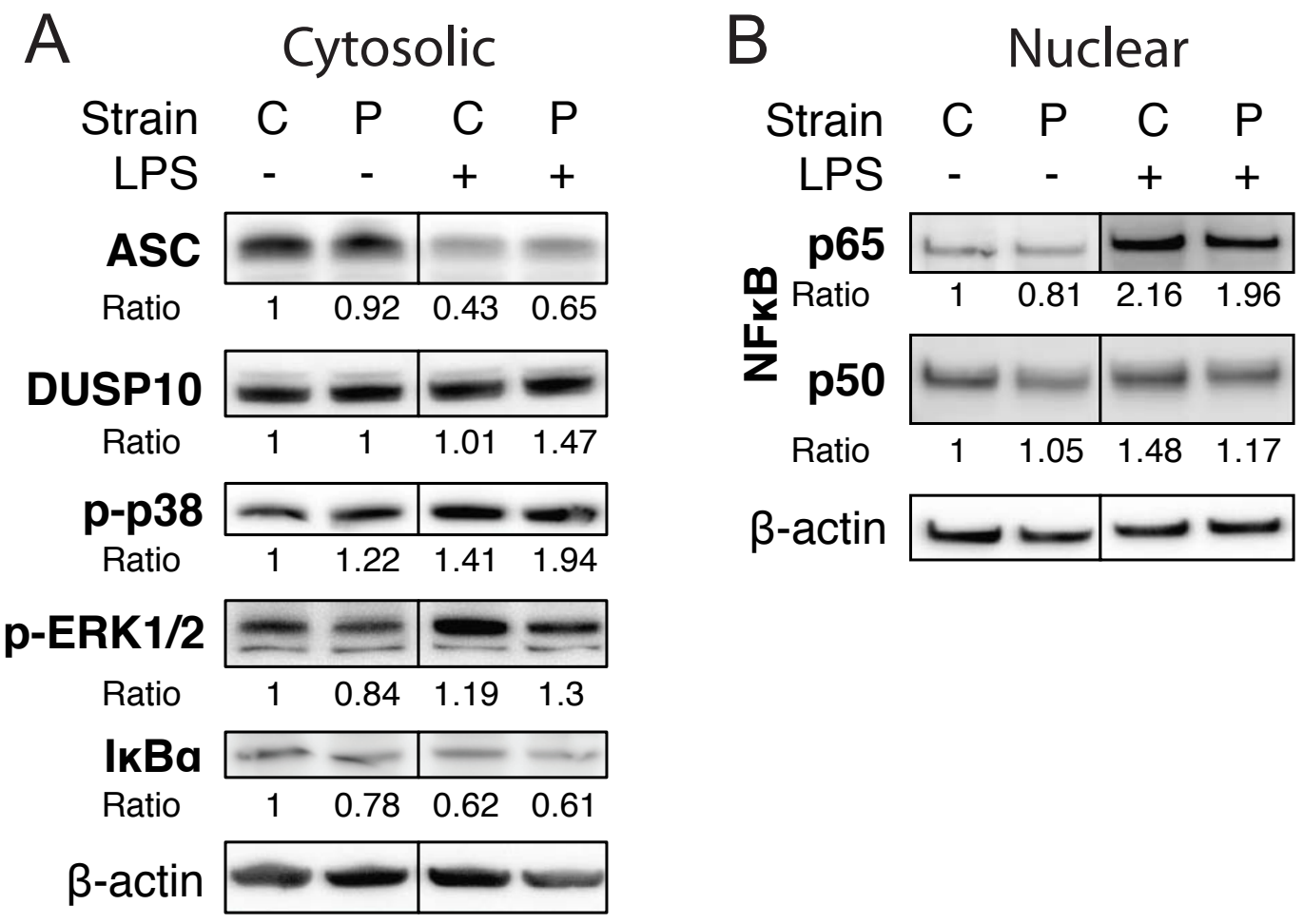

Figure 4.14 Chronic hyperuricaemia in PLT2 mice does not affect monocyte inflammatory signalling molecules with or without LPS challenge.

A: Cytosolic and B: nuclear protein from purified splenic monocytes isolated from C57 and PLT2 mice ( $n=5)$ administered LPS i.v. (40ng/kg, 1hr). Protein was analysed by western blotting. Strain: $C=C 57$ and $P=P L T 2$. Results are representative of two independent experiments. Lanes have been cropped from a single blot for display purposes.

I then analysed NLRP3 inflammasome component expression in splenic monocytes from C57 and PLT2 mice treated with or without LPS. Naïve monocytes from PLT2 mice had lower expression of pro-caspase1, caspase1 and IL-1 $\beta$ compared to C57 mice (figure 4.15). Monocytes from PLT2 mice had increased expression of NLRP3 in the absence of LPS stimulation but decreased NLRP3 (figure 4.15) following LPS stimulation, which coincided with an absence of pro-IL-1 $\beta$ expression. Densitometric analysis of the depicted western blots is presented in appendix $7.6 \mathrm{H}-\mathrm{L}$. 


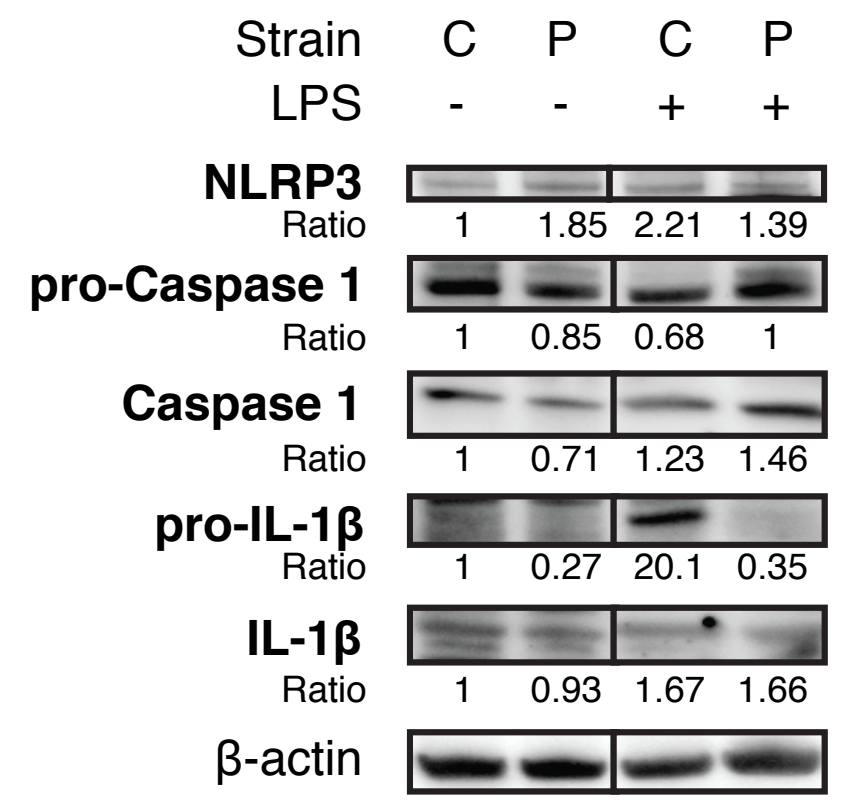

Figure 4.15 After LPS challenge PLT2 mouse monocytes have decreased NLRP3 and pro-IL-1 $\beta$ expression compared to C57.

Cytosolic protein from purified splenic monocytes isolated from C57 and PLT2 mice $(n=5)$ administered LPS i.v. (40ng/kg, $1 \mathrm{hr})$. Protein was analysed by western blotting. Strain: $C=C 57$ and $P=P L T 2$. Results are representative of two independent experiments. Lanes have been cropped from a single blot for display purposes.

These results showed that the systemic cytokine and monocyte signalling responses to LPS stimulation in chronic hyperuricaemia in the PLT2 strain differed significantly from the acute hyperuricaemia induced in C57 mice.

The PLT2 strain produced significantly more pro-inflammatory serum cytokines following LPS stimulation compared to C57 mice. Chronic hyperuricaemia had little effect on splenic monocyte inflammatory signalling with the PLT2 mice showing a small increase in MAP kinase pathway phosphorylation but not NFKB nuclear translocation. Following LPS stimulation NLRP3 expression and pro-IL-1 $\beta$ expression was decreased in PLT2 mice compared to C57.

\subsubsection{Lowering serum UA in chronic hyperuricaemia}

Next I wanted to see if lowering serum urate in the PLT2 mice could alter the inflammatory response to LPS as it had in the C57 mice. 


\section{Allopurinol}

I treated PLT2 mice with allopurinol for $3 \mathrm{hr}$ then challenged with LPS for $1 \mathrm{hr}$ and measured serum UA levels and LPS-induced serum cytokines. Interestingly, allopurinol had no effect on PLT2 mouse serum UA levels (figure 4.16A). Allopurinol treatment also had no effect on serum cytokine levels with or without LPS administration (figure 4.16B-E). There was a trend towards decreased serum IL-10 in allopurinol and LPS treated PLT2 mice versus LPS alone (figure 4.16B).
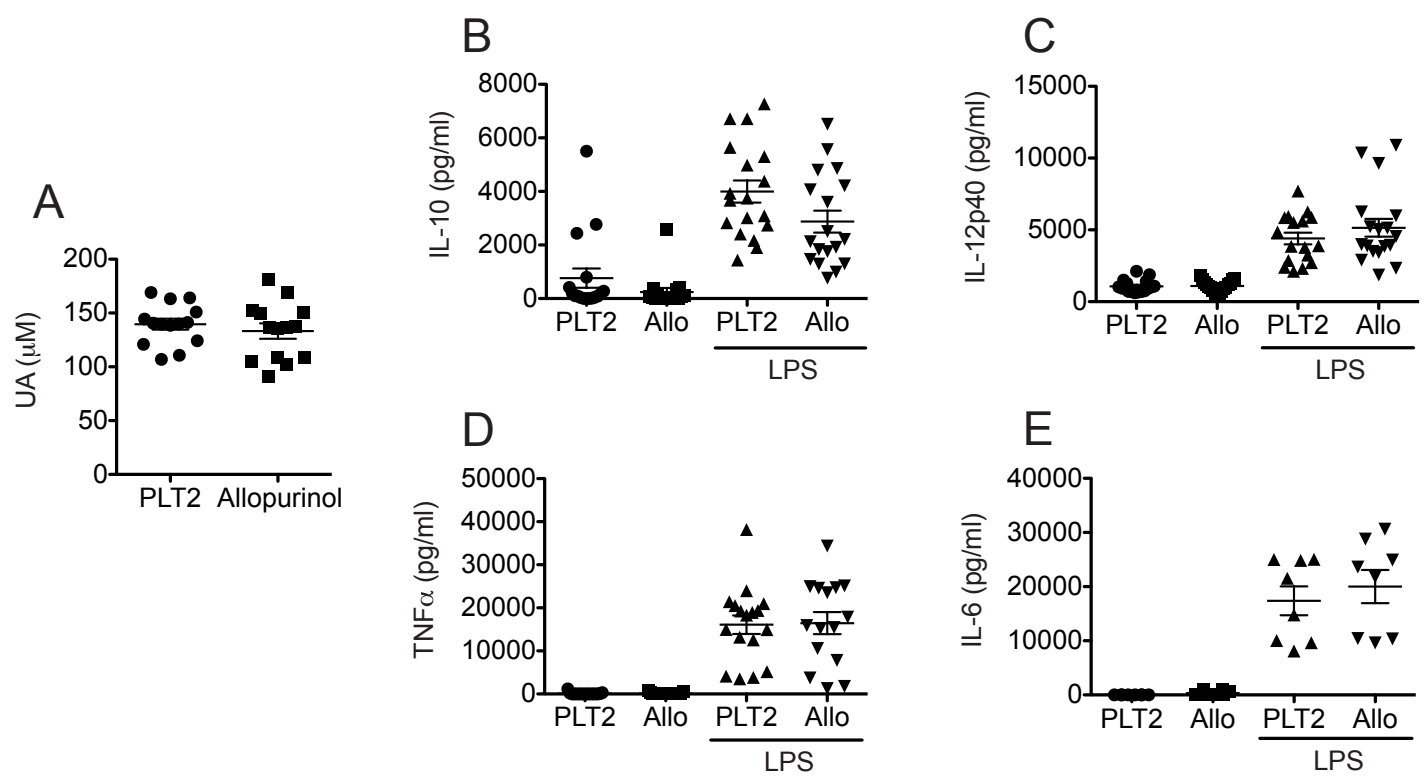

Figure 4.16 Allopurinol treatment has no effect on either serum UA or LPSinduced serum cytokines in PLT2 mice.

A: Serum UA and B-E: cytokine levels of PLT2 mice pre-treated with or without allopurinol (30mg/kg, 3hr) then administered LPS i.v. (40ng/kg, 1hr). Serum UA was measured using a fluorometric assay. Serum cytokines were measured by ELISA. Results are mean with SEM and are combined from at least two independent experiments. $A: n=15, B: n=18, C: n=18, D n=15, E: n=8$.

I then investigated protein expression in purified splenic monocytes from PLT2 mice after in vivo allopurinol treatment and LPS challenge. Expression and phosphorylation of cytosolic monocyte inflammatory proteins was not affected by allopurinol treatment in PLT2 mice (figure 4.17A). There was a 
decrease in ERK1/2 phosphorylation in allopurinol and LPS treated PLT2 mice but this pattern was not consistent in a repeat experiment indicating that more work is needed to define the effect of allopurinol on ERK1/2 phosphorylation in the PLT2 strain. Densitometric analysis showed that allopurinol treatment had no effect on p65 and p50 in unstimulated mice but decreased NFKB p50 subunit nuclear translocation with LPS treatment (figure 4.17B). Densitometric analysis of the depicted western blots is presented in appendix 7.7A-G.

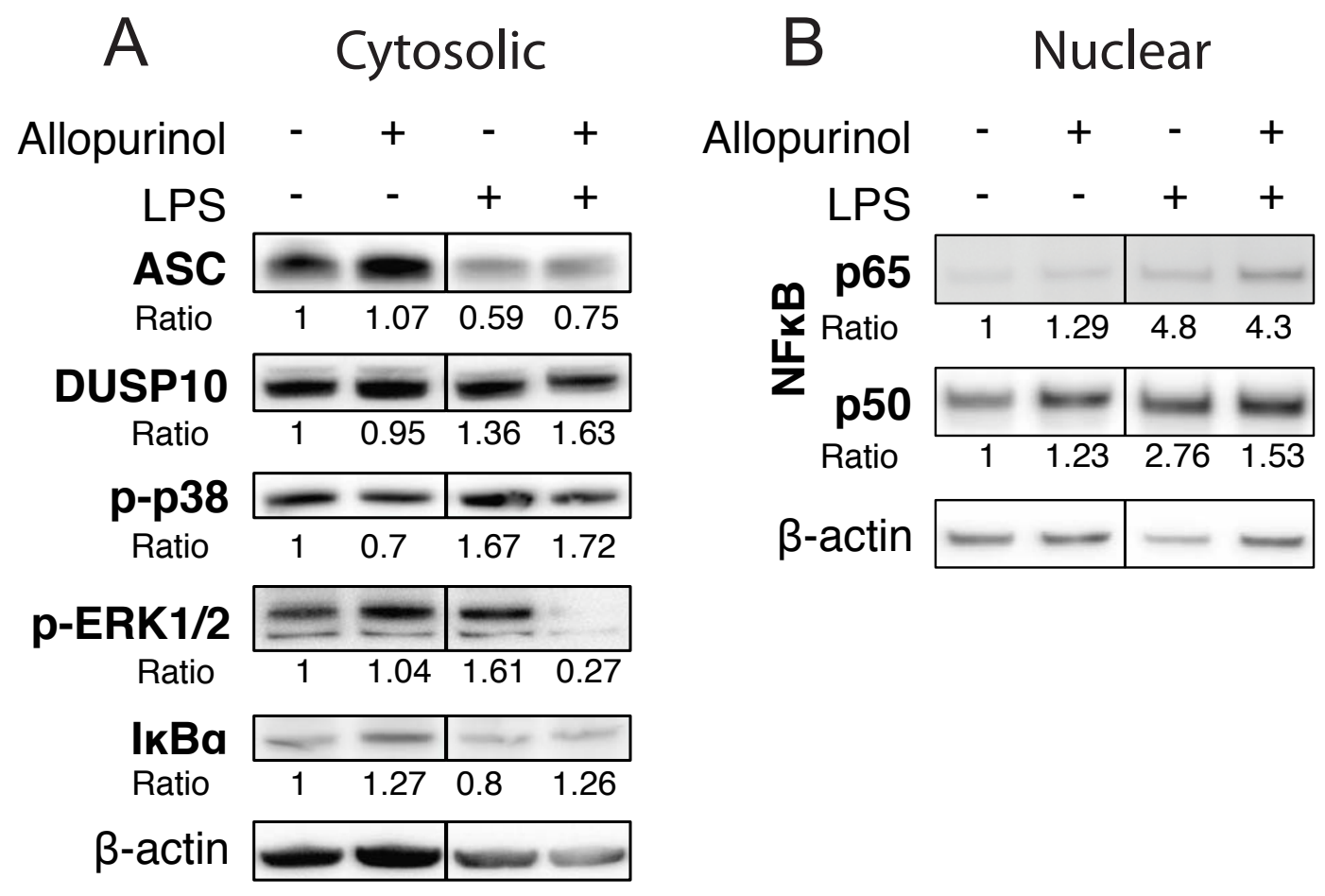

Figure 4.17 Allopurinol treatment has no effect on ASC expression in splenic monocytes from PLT2 mice.

A: Cytosolic and B: nuclear protein from purified splenic monocytes isolated from PLT2 mice (n=5) pre-treated with or without allopurinol $(30 \mathrm{mg} / \mathrm{kg}$, 3hr) then administered LPS i.v. (40ng/kg, 1hr). Protein was analysed by western blotting. Results are representative of two independent experiments. Lanes have been cropped from a single blot for display purposes. 
Allopurinol treatment increased NLRP3 protein expression in splenic monocytes from LPS treated PLT2 mice but did not affect the expression of any other NLRP3 inflammasome components (figure 4.18). Densitometric analysis of the depicted western blots is presented in appendix 7.7 H-L.

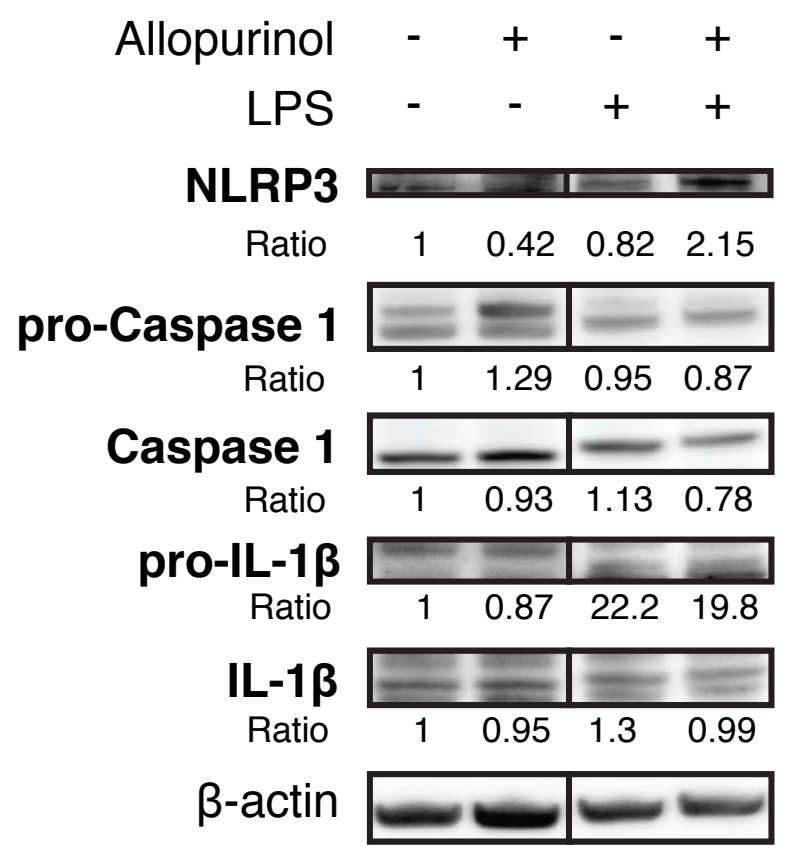

Figure 4.18 Allopurinol treatment increases NLRP3 expression in splenic monocytes from PLT2 mice.

Cytosolic protein from purified splenic monocytes isolated from PLT2 mice $(n=5)$ pretreated with or without allopurinol $(30 \mathrm{mg} / \mathrm{kg}$, 3hr) then administered LPS i.v. (40ng/kg, 1hr). Protein was analysed by western blotting. Results are representative of two independent experiments. Lanes have been cropped from a single blot for display purposes.

Acute allopurinol treatment had no effect on PLT2 mouse serum UA levels. This is likely to be the reason there was no effect of allopurinol on either the systemic cytokine levels or inflammatory monocyte signalling molecules in response to LPS challenge. 


\section{Rasburicase}

After testing allopurinol in the PLT2 strain I wanted to see if rasburicase treatment would have a similar or different effect. I treated PLT2 mice with or without rasburicase then administered LPS i.v. to investigate the effect of rasburicase on serum UA levels and inflammatory cytokine production.

Unlike allopurinol, rasburicase treatment reduced serum UA levels in PLT2 mice (figure 4.19A). However, there were no differences in LPS-induced serum cytokines between untreated and rasburicase treated PLT2 mice (figure 4.19B-E).
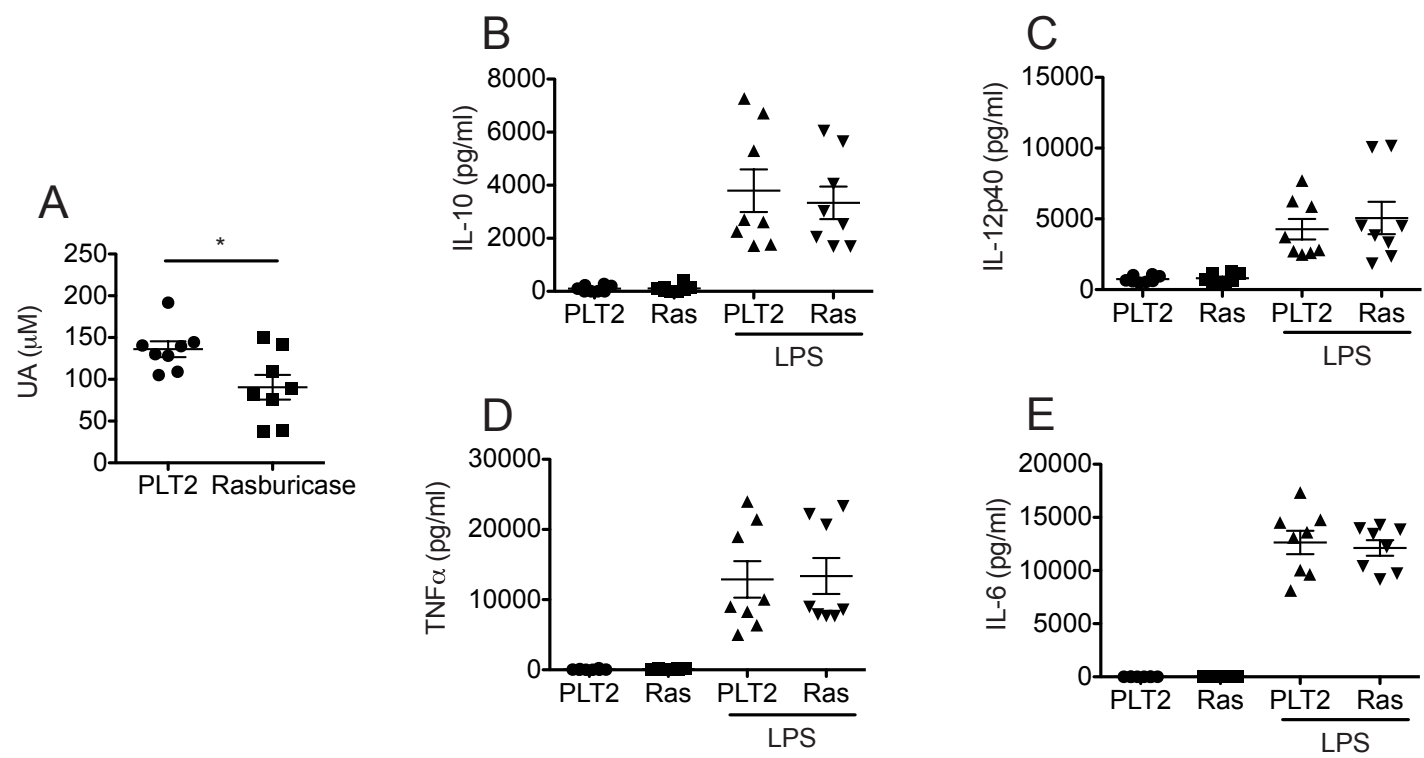

Figure 4.19 Rasburicase treatment lowers serum UA but has no effect on LPS-induced systemic inflammation in PLT2 mice.

Serum cytokine levels of C57 mice pre-treated with or without rasburicase $10.8 \mathrm{mg} / \mathrm{kg}$, $3 \mathrm{hr}$ ) then administered LPS i.v. (40ng/kg, 1hr). Serum UA was measured using a fluorometric assay. Serum cytokines were measured by ELISA. ${ }^{*} p<0.05$, student's $t$ test. Results are mean with SEM and are combined from two independent experiments. A: $n=8, B: n=8, C: n=8, D n=8, E: n=8$. 
Next I investigated inflammatory protein signalling in splenic monocytes from rasburicase treated PLT2 mice. Rasburicase treatment decreased DUSP10 expression compared to control PLT2 mice (figure 4.20A). After LPS treatment there was an increase in ERK1/2 phosphorylation in monocytes from rasburicase treated PLT2 mice (figure 4.20A). Rasburicase treatment increased NFKB p65 subunit nuclear translocation in splenic monocytes following LPS challenge (figure 4.20B). Densitometric analysis of the depicted western blots is presented in appendix 7.8A-G.

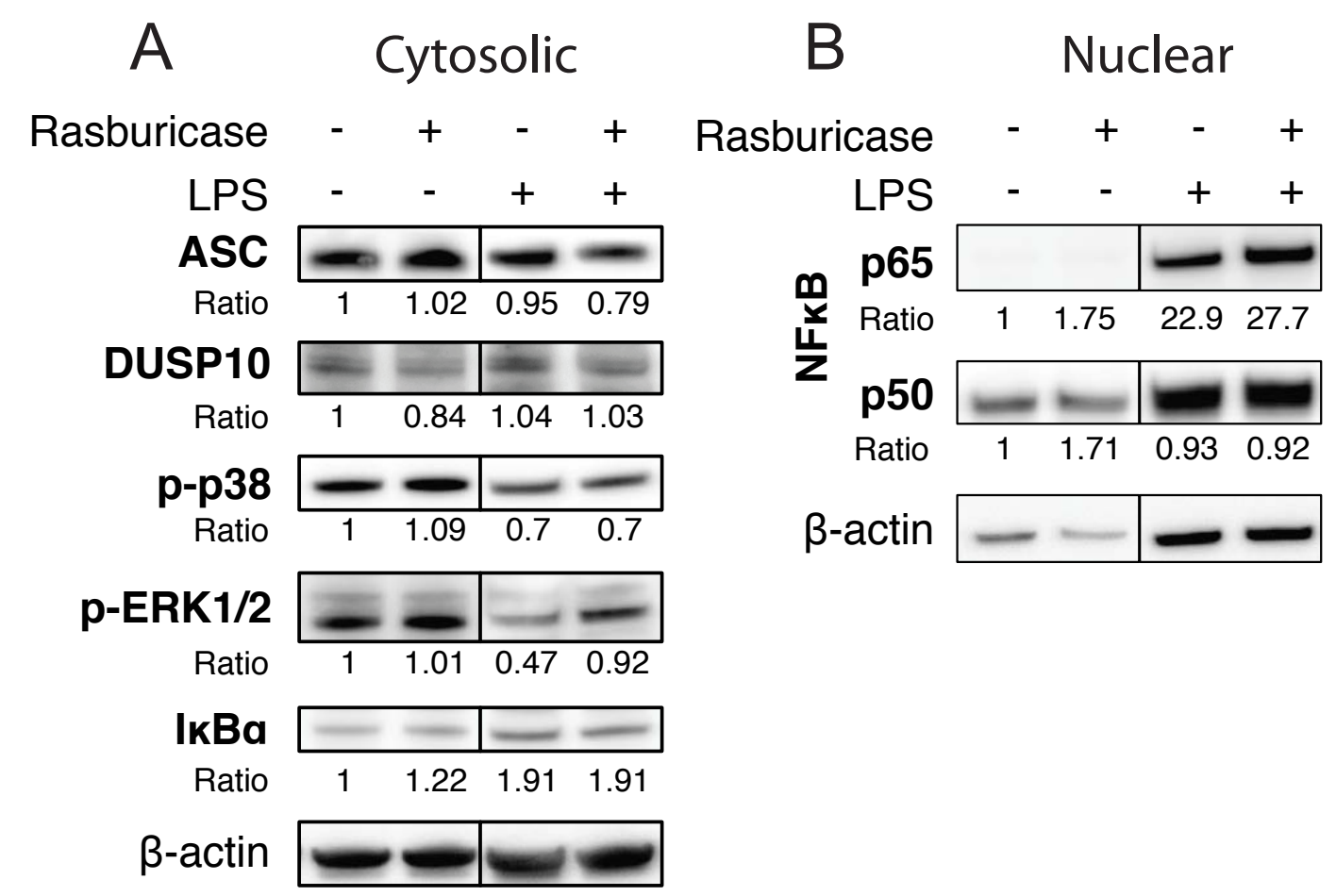

Figure 4.20 Rasburicase treatment increases $p$-ERK1/2 and increases NFKB p65 subunit nuclear translocation in splenic monocytes from PLT2 mice.

A: Cytosolic and B: nuclear protein from purified splenic monocytes isolated from PLT2 mice $(n=5)$ pre-treated with or without rasburicase $(0.8 \mathrm{mg} / \mathrm{kg}$, $3 \mathrm{hr})$ then administered LPS i.v. (40ng/kg, 1hr). Protein was analysed by western blotting. Results are representative of two independent experiments. Lanes have been cropped from a single blot for display purposes. 
I analysed the expression of NLRP3 inflammasome components in rasburicase treated PLT2 mice with and without LPS challenge. Monocyte expression of pro-caspase 1 and pro-IL-1 $\beta$ were increased in rasburicase treated PLT2 mice administered LPS (figure 4.21). Densitometric analysis of the depicted western blots is presented in appendix $7.8 \mathrm{H}-\mathrm{L}$.

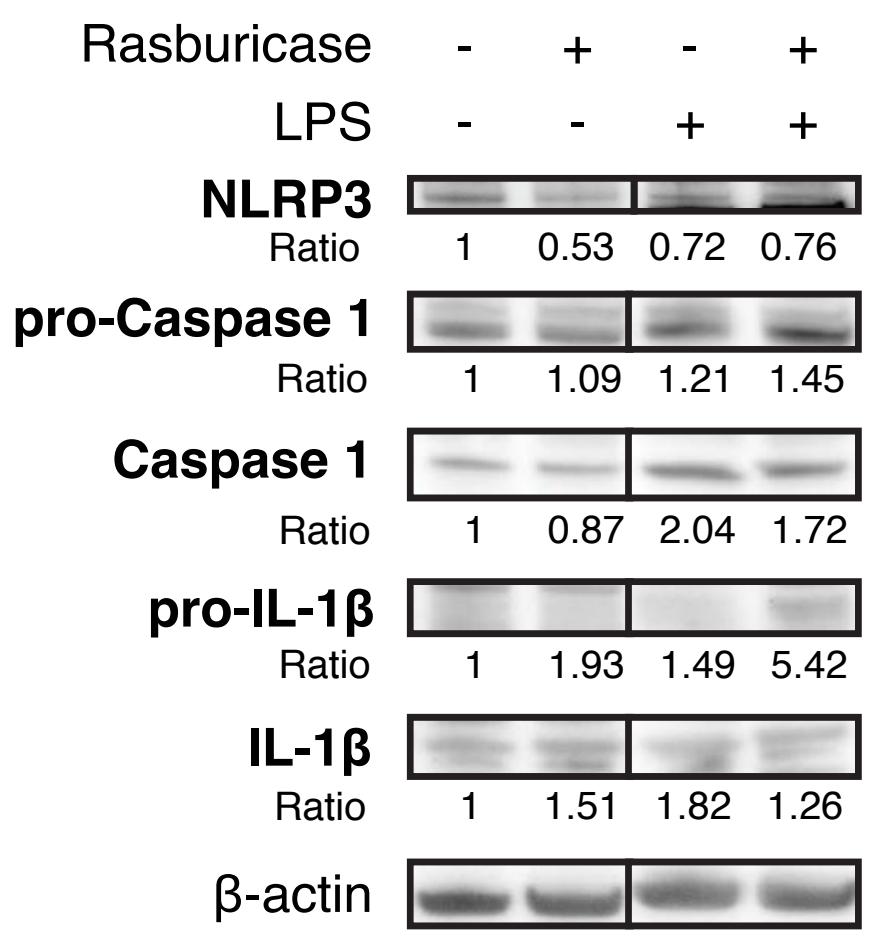

Figure 4.21 Rasburicase treatment increases pro-caspase1 and pro-IL-1 $\beta$ expression in splenic monocytes from PLT2 mice.

Cytosolic protein from purified splenic monocytes isolated from PLT2 mice $(n=5)$ pretreated with or without rasburicase $(0.8 \mathrm{mg} / \mathrm{kg}$, 3hr) then administered LPS i.v. $(40 \mathrm{ng} / \mathrm{kg}, 1 \mathrm{hr})$. Protein was analysed by western blotting. Results are representative of two independent experiments. Lanes have been cropped from a single blot for display purposes.

These results demonstrated that rasburicase was effective at reducing serum UA levels in PLT2 mice, but had little effect on the monocyte inflammatory signalling profile with increased ERK1/2 phosphorylation and NFKB p65 nuclear translocation. Also, this reduction in serum UA did not translate into an increase in systemic inflammatory cytokines or a decrease in serum IL-10 after LPS administration. 


\subsection{Summary}

In this chapter I explored how changes to systemic UA levels influence the acute inflammatory response to a non-lethal dose of LPS in vivo. My in vitro results from Chapter 3 provided an experimental platform that I used to investigate the systemic inflammatory cytokine response and inflammatory signalling in splenic monocytes following in vivo LPS challenge. I started by investigating the effect of acute hyperuricaemia and found that, like my in vitro human monocyte results, raising UA levels reduced the inflammatory cytokine response to LPS and increased IL-10. Splenic monocytes from LPSstimulated mice with acute hyperuricaemia had a decreased inflammatory signalling profile similar to human blood monocytes with in vitro hyperuricaemia.

Allopurinol and rasburicase both effectively lowered serum UA levels in C57 mice but only allopurinol treatment resulted in increased inflammatory serum cytokines and decreased IL-10 in response to LPS administration. Lowering serum UA with allopurinol or rasburicase had differential effects on monocyte inflammatory signalling pathways where both treatments increased inflammatory NFKB signalling but only rasburicase increased ASC expression. This indicates that the mechanism of urate lowering may be an important consideration in determining the immunological outcome.

As a model of chronic hyperuricaemia I used the PLT2 mouse strain and compared the systemic inflammatory responses of PLT2 and C57 mice to LPS challenge. There was an increase in IL-10 and pro-inflammatory serum cytokines in the PLT2 strain with increased MAP kinase phosphorylation. However, NLRP3 and pro-IL-1 $\beta$ expression were reduced in the PLT2 strain. The discrepancy between the results observed with acute vs chronic hyperuricaemia could be a result of the genetic mutation in the PLT2 mouse or may represent differences between the immunological effects of short-term and long-term hyperuricaemia.

Finally I treated the PLT2 mice with allopurinol or rasburicase and found that acute allopurinol treatment had no effect on serum UA levels in the PLT2 strain. Allopurinol also had no effect on serum cytokines or monocyte signalling during LPS-induced systemic inflammation, demonstrating that in 
the PLT2 mouse, acute allopurinol treatment did not affect cytokine and monocyte responses to LPS in the absence of UA lowering. However, Rasburicase treatment, which lowered serum UA, did not alter serum cytokines but did increase the monocyte inflammatory signalling profile via increased ASC expression, decreased DUSP10 and increased NFKB p65 nuclear translocation. Indicating again that the mechanism of urate lowering may be an important factor in determining the immunological effect of soluble UA.

Taken together, these results confirmed that, like acute in vitro hyperuricaemia, acute in vivo hyperuricaemia suppressed inflammatory cytokines and monocyte inflammatory signalling. The effect of allopurinol and rasburicase on systemic and monocytic responses to LPS in C57 mice provided proof of concept to support the development of a clinical study to investigate urate-lowering therapy. Chapter 5 investigates how urate-lowering therapy modulates the response of human blood monocytes to immune stimuli. 
Chapter 5:

Urate-Lowering Therapy Study 



\section{Urate-lowering therapy study}

\subsection{Introduction}

Urate-lowering therapy (ULT) is prescribed primarily for the treatment and prevention of gouty arthritis ${ }^{83}$. However, it is also used during chemotherapy of haematological cancers to combat tumour lysis syndrome, where extensive cell death causes an acute spike in serum UA that can result in acute kidney injury ${ }^{197}$. In the previous two chapters I used in vitro soluble UA treatment and in vivo models of hyperuricaemia in combination with ULT to investigate the role of soluble UA in modulating LPS-induced inflammation. The overarching results were that increases in soluble UA suppressed monocyte responses to LPS and enhanced IL-10 production and that elements of this immune suppression could be reversed with ULT.

The next stage was to test whether the suppressive effects of soluble UA could be reversed by ULT in the treatment of patients with hyperuricaemia.

There are three different mechanisms of urate lowering: xanthine oxidoreductase inhibition, recombinant uricase treatment and uricosurics. Allopurinol, a xanthine oxidoreductase inhibitor, is the first line ULT for hyperuricaemia associated with gout, which made it the best option for an initial proof of concept clinical study.

A clinical study had been designed to examine the kinetics of allopurinol metabolism in patients with hyperuricaemia and gout and I expanded the scope of this study to examine the effect of ULT on the phenotype of blood monocytes and their inflammatory function.

\subsection{Aim}

The aim of this chapter was to determine whether ULT increases the inflammatory cytokine and signalling responses of human blood monocytes. 


\subsection{Results}

\subsubsection{Demographic data}

15 patients were sequentially recruited as described in the materials and methods (section 2.2.15). The study participants were $80 \%$ male and had a mean age of 62 (table 5.1) consistent with hyperuricaemia being more common in males and the ageing population ${ }^{35}$. Most patients were classed as overweight (BMI 25-29.9) or obese (BMI $\geq 30$ ) with a mean BMI of $30.13 \mathrm{~kg} / \mathrm{m}^{2}$. Several patients also had metabolic syndrome related comorbidities such as diabetes, hypertension and ischemic heart disease, which is consistent with the BMI data and the well-established association between hyperuricaemia and metabolic syndrome (table 5.1). Anti-inflammatory medications are often prescribed as prophylaxis or treatment for the pain and inflammation associated with gout. Therefore, changes to patient anti-inflammatory medications were also recorded at baseline and day 28 (table 5.1). Patient comorbidities and medications could influence the phenotype and function of immune cells and should be considered when interpreting the results of this study. However, given the small sample size and preliminary nature of this study I was unable to exclude patients with comorbidities or those that used anti-inflammatory medications. 
Table 5.1 Serum UA levels, demographic data, comorbidities and medication.

\begin{tabular}{|c|c|c|c|c|c|c|c|c|c|c|c|c|c|c|}
\hline Study No. & $\begin{array}{c}\text { Experimental } \\
\text { No. }\end{array}$ & $\begin{array}{l}\text { SUA } \\
\text { Day } 0 \\
\end{array}$ & $\begin{array}{c}\text { sUA } \\
\text { Day } 1\end{array}$ & $\begin{array}{c}\text { SUA } \\
\text { Day } 28 \\
\end{array}$ & $\begin{array}{c}\begin{array}{c}\text { Allopurinol } \\
\text { (mg/day) }\end{array} \\
\end{array}$ & Age & Sex & BMI & $\begin{array}{l}\text { Metabolic syndrome } \\
\text { related comorbidities }\end{array}$ & Predinsone & NSAIDs & s Colchicine & $\begin{array}{l}\text { Cytokine } \\
\text { analysis }\end{array}$ & $\begin{array}{c}\text { Protein } \\
\text { analysis }\end{array}$ \\
\hline AUHG03 & 1 & 0.58 & 0.58 & 0.51 & 50 & 69 & $f$ & 22.34 & None & - & - & - & LPS & - \\
\hline AUHG04 & 2 & 0.64 & 0.61 & 0.57 & 100 & 51 & $\mathrm{~m}$ & 33.88 & None & - & - & - & LPS & - \\
\hline AUHG05 & 3 & 0.44 & 0.44 & 0.43 & 100 & 64 & $\mathrm{~m}$ & 37.45 & $\begin{array}{l}\text { Diabetes, hypertension, } \\
\text { hypercholesterolemia, } \\
\text { IHD, renal impairment }\end{array}$ & - & - & - & LPS & - \\
\hline AUHG06 & 4 & 0.46 & 0.45 & 0.46 & 50 & 82 & $f$ & 32.04 & $\begin{array}{l}\text { Hypertension, } \\
\text { hypercholesterolemia, } \\
\text { IHD, renal impairment }\end{array}$ & + & - & + & LPS & - \\
\hline AUHG07 & 5 & 0.53 & 0.54 & 0.51 & 50 & 65 & $\mathrm{~m}$ & 32.71 & None & - & - & - & LPS & - \\
\hline AUHG08 & 6 & 0.56 & 0.53 & 0.48 & 100 & 34 & $f$ & 33.17 & Renal impairment & - & - & - & LPS & - \\
\hline AUHG09 & 7 & 0.57 & 0.61 & 0.47 & 100 & 60 & $\mathrm{~m}$ & 45.9 & $\begin{array}{l}\text { Diabetes, hypertension, } \\
\text { hypercholesterolemia }\end{array}$ & - & - & - & $\begin{array}{l}\text { LPS and } \\
\text { MSU }\end{array}$ & LPS \\
\hline AUHG10 & 8 & 0.45 & 0.41 & 0.44 & 100 & 46 & $\mathrm{~m}$ & 35.8 & None & - & + & - & $\begin{array}{l}\text { LPS and } \\
\text { MSU }\end{array}$ & - \\
\hline AUHG11 & 9 & 0.54 & 0.49 & 0.45 & 100 & 56 & $\mathrm{~m}$ & 26.28 & None & - & + & - & $\begin{array}{c}\text { LPS and } \\
\text { MSU }\end{array}$ & MSU \\
\hline AUHG12 & 10 & 0.69 & 0.56 & 0.53 & 50 & 80 & $\mathrm{~m}$ & 24.34 & $\begin{array}{l}\text { Hypertension, } \\
\text { hypercholesterolemia, } \\
\text { IHD, renal impairment }\end{array}$ & + & + & + & $\begin{array}{l}\text { LPS and } \\
\text { MSU }\end{array}$ & LPS \\
\hline AUHG13 & 11 & 0.51 & 0.49 & 0.51 & 50 & 60 & $\mathrm{~m}$ & 26.13 & $\begin{array}{l}\text { Hypertension, } \\
\text { hypercholesterolemia, } \\
\text { renal impairment }\end{array}$ & + & - & - & $\begin{array}{l}\text { LPS and } \\
\text { MSU }\end{array}$ & MSU \\
\hline AUHG14 & 12 & 0.46 & 0.41 & 0.39 & 100 & 63 & $\mathrm{~m}$ & 28.91 & None & - & + & - & $\begin{array}{l}\text { LPS and } \\
\text { MSU }\end{array}$ & LPS \\
\hline AUHG15 & 13 & 0.66 & 0.63 & 0.51 & 50 & 69 & $\mathrm{~m}$ & 23.16 & $\begin{array}{l}\text { Hypertension, renal } \\
\text { impairment }\end{array}$ & + & - & - & $\begin{array}{l}\text { LPS and } \\
\text { MSU }\end{array}$ & MSU \\
\hline AUHG16 & 14 & 0.48 & 0.44 & 0.39 & 100 & 71 & $\mathrm{~m}$ & 25.91 & $\begin{array}{l}\text { Hypercholesterolemia, } \\
\text { IHD }\end{array}$ & - & + & - & $\begin{array}{l}\text { LPS and } \\
\text { MSU }\end{array}$ & LPS \\
\hline \multirow[t]{2}{*}{ AUHG17 } & 15 & 0.54 & 0.50 & 0.49 & 100 & 63 & $\mathrm{~m}$ & 23.96 & IHD & + & - & - & $\begin{array}{l}\text { LPS and } \\
\text { MSU }\end{array}$ & MSU \\
\hline & $\begin{array}{l}\text { Mean } \\
\text { (SD) }\end{array}$ & $\begin{array}{c}0.54 \\
(0.08) \\
\end{array}$ & $\begin{array}{c}0.51 \\
(0.07)^{\star}\end{array}$ & $\begin{array}{c}0.48 \\
(0.05)^{\star \star \star} \\
\end{array}$ & & $\begin{array}{c}62.2 \\
(12.3) \\
\end{array}$ & & $\begin{array}{l}30.13 \\
(6.54) \\
\end{array}$ & & & & & & \\
\hline
\end{tabular}

Individual and mean values (SD) for patient serum UA (sUA) age and body mass index (BMI). Several patients also suffered from metabolic syndrome related disorders and were taking anti-inflammatory medication. Cytokine and protein analysis columns denote which treatment patient monocytes were stimulated with for cytokine and protein analysis. ${ }^{*} p<0.05$ patient serum UA levels at day 0 vs day 1 and ${ }^{* * *} p<0.001$ day 0 vs day 28 , paired samples t-test. Patient AUHG05 started allopurinol before the study began. 


\subsubsection{Patient serum UA levels}

All the patients were hyperuricaemic (serum $U A \geq 0.42 \mathrm{mM}$ ) at the beginning of the study with a mean serum UA level of $0.54 \mathrm{mM}$ (table 5.1 ). ULT with allopurinol significantly reduced serum UA levels by day 1 with further significant reduction by day 28 (table 5.1). The majority of patients were still hyperuricaemic at day 28 and none had reached the target level of $<0.36 \mathrm{mM}$, which may be due to the low dose of allopurinol (50-100mg/day).

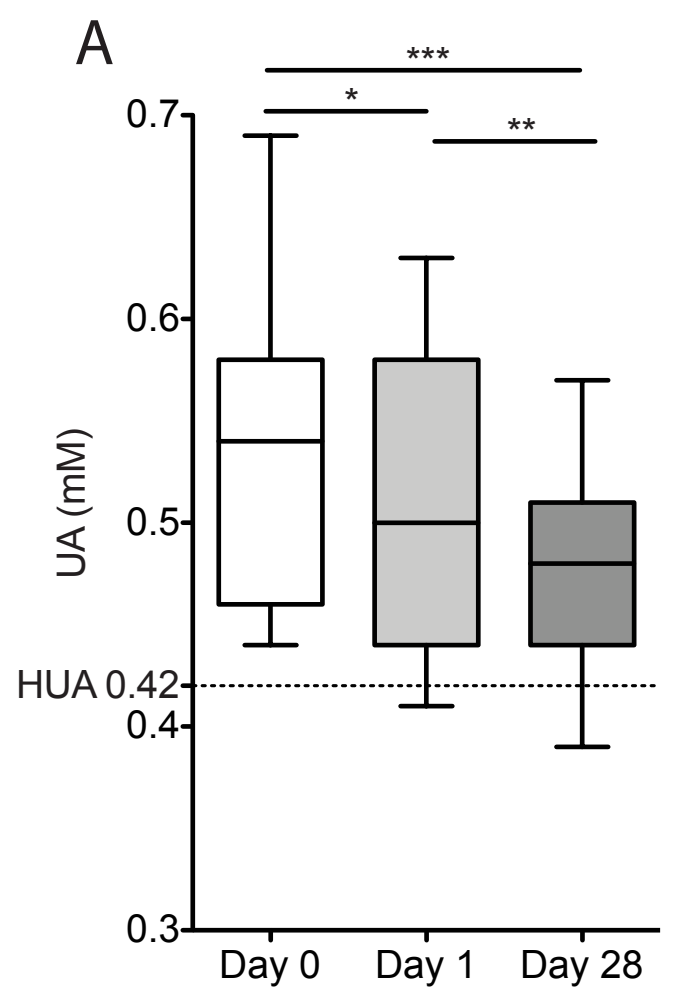

Figure 5.1 ULT reduces patient serum UA levels.

Patient serum UA levels measured on day 0 , day 1 and day 28 of ULT. HUA $=$ Hyperuricaemia. ${ }^{*} p<0.05,{ }^{* *} p<0.01,{ }^{* * *} p<0.001$, paired samples $t$-test. Graph displays median, upper and lower quartiles, maximum and minimum. 


\subsubsection{Blood monocyte percentages}

There are two main monocyte subsets in the blood, the CD14+ circulating monocytes and the CD14+/CD16+ patrolling monocytes, both of which are CD11b positive ${ }^{119}$. The Harper group has shown that hyperuricaemia positively correlates with increased serum CCL2, a major monocyte chemokine, and that serum CCL2 in turn positively correlates with CD14+ blood monocyte percentages ${ }^{128}$. To determine whether ULT altered the percentages of the two blood monocyte subsets I stained whole white blood cell (WBC) preparations with antibodies for CD14 and CD16 and analysed the populations by flow cytometry. Figure 5.2 is a representative flow plot showing the two blood monocyte populations (CD14+ and CD14+/CD16+) and the larger neutrophil population (CD16+).

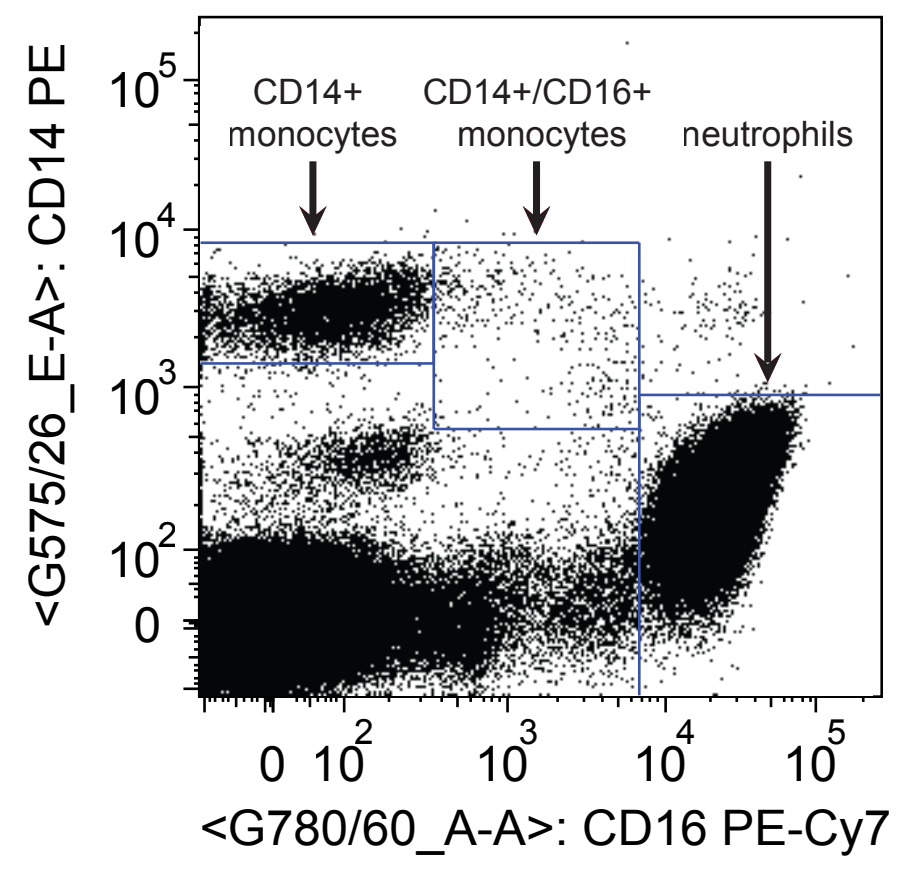

Figure 5.2 Representative flow plot of CD14 and CD16 stained white blood cells (WBC).

Isolated WBC were stained with fluorescent antibodies for CD14 and CD16 then analysed by flow cytometry. 
ULT had no significant effect on the overall percentages of blood monocytes or neutrophils (figure 5.3a-c). I also analysed the cell percentages from each patient versus the patient's serum UA level at each time point. This analysis produced a positive correlation between serum UA and the percentage of CD14+ blood monocytes (figure 5.3d). There was a trend towards increased CD14+/CD16+ monocytes with increasing serum UA and there was no association between serum UA and neutrophils (figure $5.3 e-f$ ).
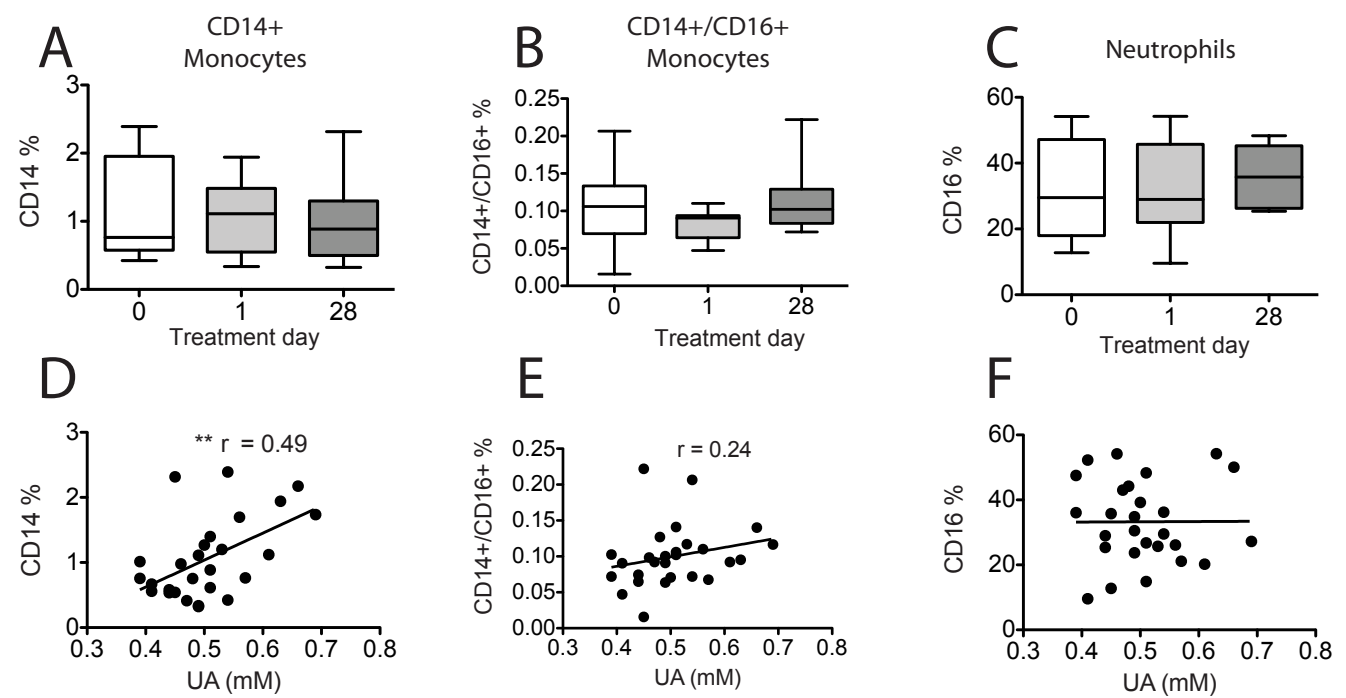

Figure 5.3 Monocyte and neutrophil percentages before and after ULT. Patient WBC $(n=8)$ were stained with fluorescent antibodies for CD14, CD16 and $C D 11 b$ and cell percentages were analysed by flow cytometry. Percentage of $A$ : CD14+ monocytes, B: CD14+/CD16+ monocytes and C: CD16+ neutrophils. Graphs display median, upper and lower quartiles, maximum and minimum. Correlation analysis of D: CD14+ monocytes, E: CD14+/CD16+ monocytes and F: CD16+ neutrophils from each patient at each time point compared to patient serum UA at each time point. ${ }^{* *} p<0.01$, Pearson's correlation coefficient.

\subsubsection{Serum chemokines and cytokines}

I measured the levels of chemokines and cytokines in patient serum before and after ULT. I found that ULT had no effect on serum CCL2 levels (figure 5.4a) and there was no association when serum CCL2 from each patient at each time point was analysed alongside serum UA (figure 5.4b). This indicated that serum UA levels and blood monocyte percentages may not be 
so tightly linked to CCL2 as we had first supposed. I also measured IL-8, CCL4, CCL5, CCL7, IL-10, IL-12p40, TNF $\alpha, I L-6, I L-1 \beta$ and IL-1Ra. There was a negative correlation between serum IL-10 and IL-8 compared to serum UA levels but ULT had no effect on the other serum cytokines or chemokines measured (appendices 7.10 and 7.11).
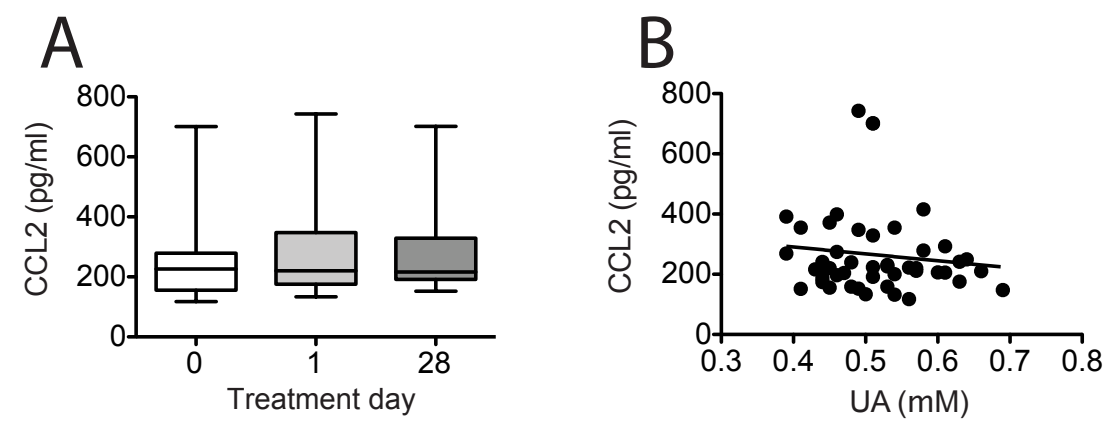

Figure 5.4 There is no relationship between ULT or serum UA levels and serum CCL2.

Patient CCL2 levels ( $n=15)$ were measured in patient serum by multiplex bead array. A: Serum CCL2 levels grouped by treatment day. Graph displays median, upper and lower quartiles, maximum and minimum. B: Serum CCL2 levels compared to patient serum UA at each time point.

\subsubsection{Adhesion molecule expression}

CD11b is highly expressed on circulating monocytes and is an important adhesion molecule involved in monocyte diapedesis through the blood vessel endothelium ${ }^{198}$. We have shown previously that monocytes from patients with hyperuricaemia and gout express more CD11b than monocytes from healthy controls $^{128}$. Therefore, I analysed the mean fluorescent intensity (MFI) of CD11b expression of the two monocyte subsets and neutrophils from each patient by flow cytometry to determine whether ULT reduced CD11b expression.

Figure 5.5a-c displays representative histograms of the downward shift in CD11b expression in monocytes and neutrophils at this time point. Analysis of the combined results showed that there was a trend towards decreased CD11b expression in CD14+ monocytes following ULT (figure 5.5d). CD11b 
expression on CD14+/CD16+ monocytes and CD16+ neutrophils at day 28 of ULT was significantly lower compared to day 0 (figure $5.5 e-f$ ). There was a positive correlation between monocyte CD11b expression and serum UA levels in all three cells subsets (figure $5.5 \mathrm{~g}-\mathrm{i}$ ). These results indicated that the increased monocyte CD11b expression, associated with increased serum UA, levels could be reversed by ULT.
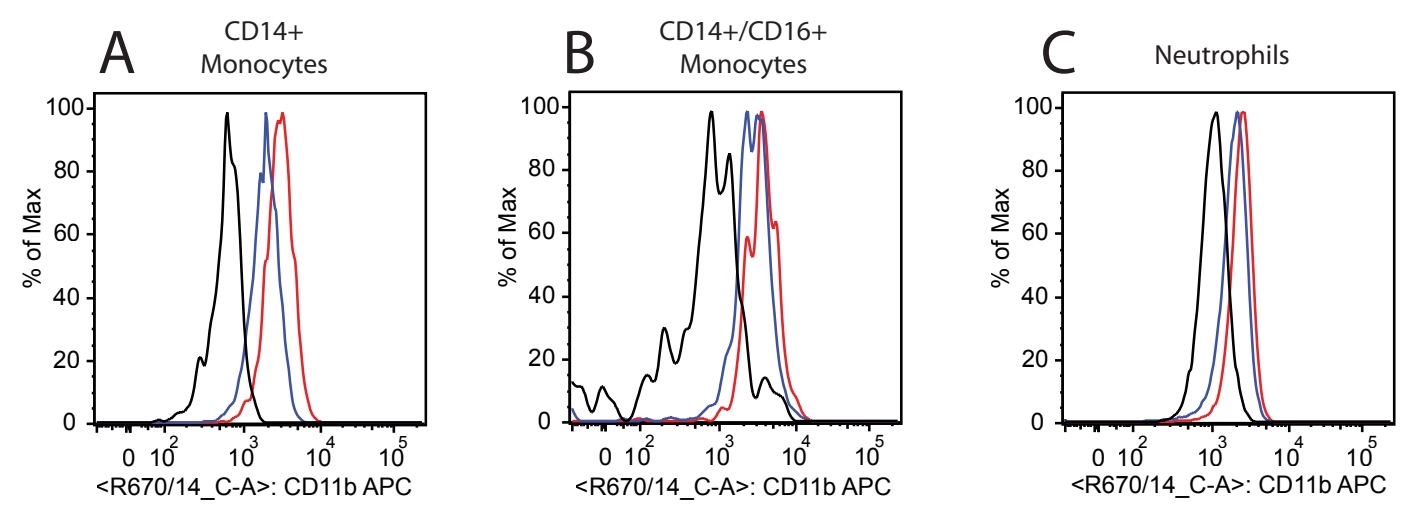

Day 0: Red Day 1: Blue Day 28: Black
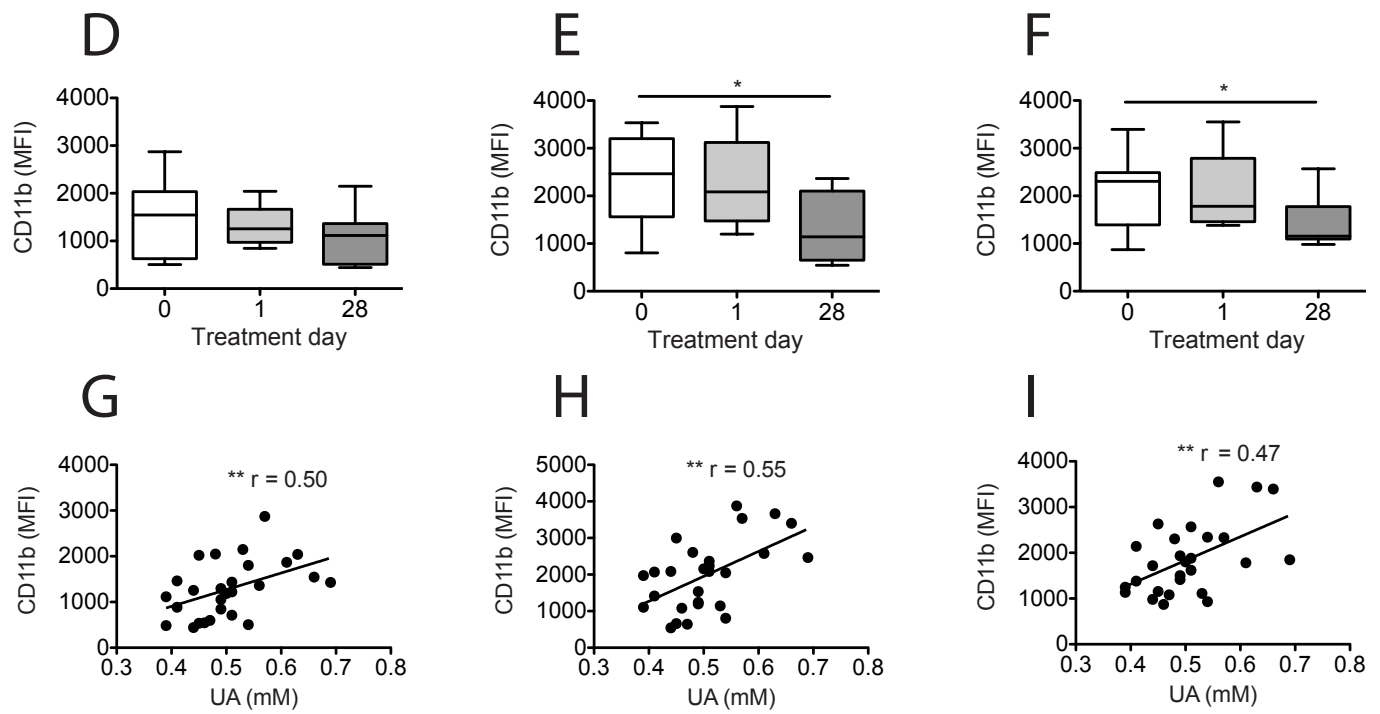

Figure 5.5 Monocyte and neutrophil CD11b expression decreases with ULT. Patient WBC ( $n=8)$ were stained with fluorescent antibodies for CD14, CD16 and $C D 11 b$, and mean fluorescent intensity (MFI) was analysed by flow cytometry. Representative histograms of $C D 11 b$ expression on $A: C D 14+$ monocytes, $B$ : CD14+/CD16+ monocytes and C: CD16+ neutrophils. CD11b expression of $D$ : CD14+ monocytes, E: CD14+/CD16+ monocytes and F: CD16+ neutrophils. Correlation analysis of CD11b expression in G: CD14+ monocytes, $\mathrm{H}$ : CD14+/CD16+ monocytes and I: CD16+ neutrophils from each patient at each time point compared to patient serum UA at each time point. ${ }^{*} p<0.05,{ }^{* *} p<0.01, D-F$ : paired samples t-test, graphs display median, upper and lower quartiles, maximum and minimum. G-I: ${ }^{* *} p<0.01$, Pearson's correlation coefficient. 
Next I investigated the effect of ULT on the expression of adhesion molecules on CD14+ monocytes after LPS stimulation. I used flow cytometry to measure the MFI of CD11b and ICAM1 on isolated blood monocytes following 18hr culture with or without LPS stimulation.

Figure 5.6a and $\mathrm{b}$ are representative histograms of CD11b and ICAM1 expression respectively displaying the reduced adhesion molecule expression on LPS-treated monocytes at day 1 and a further reduction at day 28. Analysis of the combined results from each patient at each time point showed a strong trend towards decreased CD11b and ICAM1 expression following ULT in unstimulated monocytes (figure 5.6c-d, Unstimulated). Expression of CD11b and ICAM1 was significantly lower in LPS treated monocytes after 28 days of ULT (figure $5.6 c-d$, LPS).
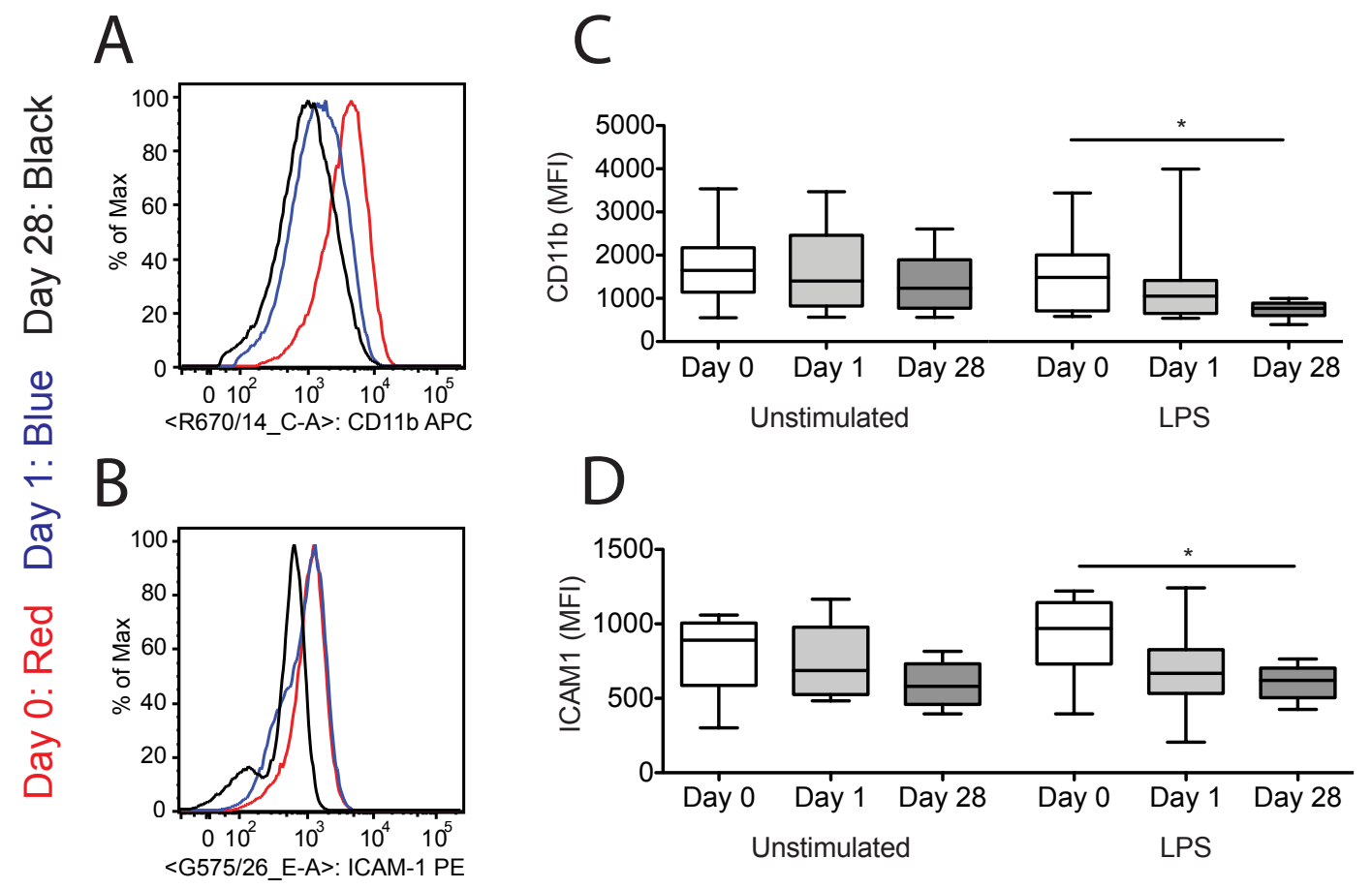

Figure 5.6 ULT reduces expression of monocyte adhesion molecules.

Patient blood monocytes $(n=9)$ were isolated and cultured for $18 \mathrm{hr}$ with or without LPS stimulation (100ng/ml), stained with fluorescent antibodies. $A$ and C: CD11b and $B$ and D: ICAM1 mean fluorescent intensity (MFI) was analysed by flow cytometry. ${ }^{*} p<0.05$, paired samples $t$-test. $C$-D: Graphs display median, upper and lower quartiles, maximum and minimum. 


\subsubsection{Monocyte cytokine production following ULT}

I demonstrated in Chapter 4 that murine ULT enhanced serum proinflammatory cytokines and reduced IL-10 following in vivo LPS challenge. Therefore, I wanted to determine whether lowering serum UA in a clinical context resulted in an increase in pro-inflammatory cytokine production from human blood monocytes. To do this I cultured blood monocytes purified before and after ULT with or without LPS or MSU for 18hr and measured the production of multiple cytokines (IL-10, IL12p40, TNF $\alpha$, IL-6, IL-1 $\beta$ and IL$1 \mathrm{Ra})$ in the culture supernatants.

ULT had no effect on background monocyte IL-10 production (figure 5.7a, Unstimulated). 28 days of ULT significantly reduced monocyte IL-10 production in response to LPS (figure 5.7a, LPS). As expected MSU did not stimulate monocytes to produce IL-10 above background levels (figure 5.7a, MSU). ULT did not alter IL12p40 production in unstimulated or stimulated monocytes (figure 5.7b).
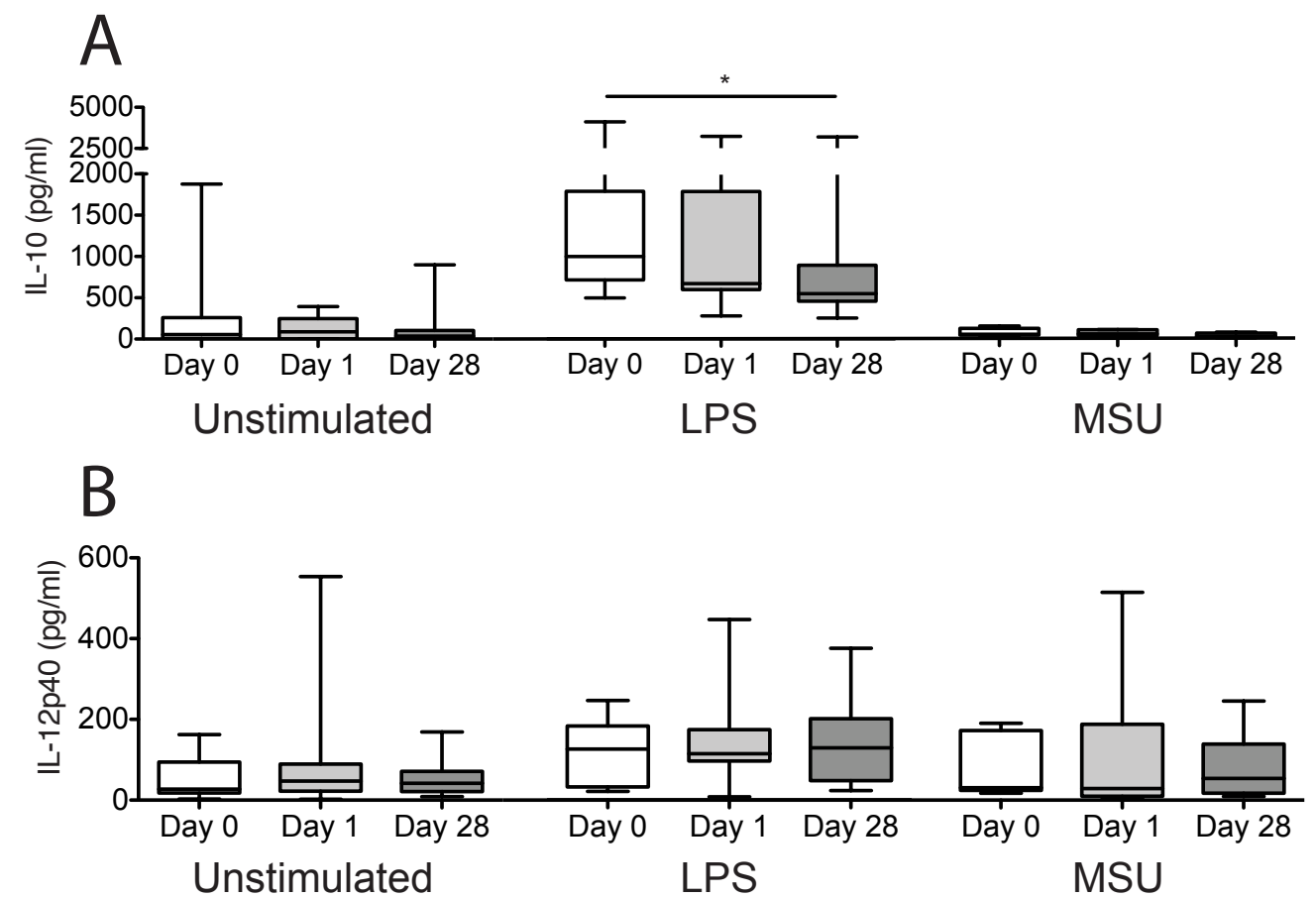

Figure 5.7 ULT reduces LPS-induced monocyte IL-10 production.

Patient blood monocytes were isolated and cultured for $18 \mathrm{hr}$ unstimulated $(n=15)$ or with LPS (100ng/mI $n=15)$ or MSU $(0.5 \mathrm{mg} / \mathrm{ml} n=7)$ stimulation. A: IL-10 and B: IL$12 p 40$ in cell culture supernatants were analysed by ELISA or multiplex bead array. ${ }^{*} p<0.05$, paired samples $t$-test. Graphs display median, upper and lower quartiles, maximum and minimum. 
I also analysed monocyte cytokine production in relation to each patient's serum UA levels at each time point. There were no clear associations between IL-10 or IL-12p40 production and serum UA levels in unstimulated or stimulated monocytes (figure 5.8).
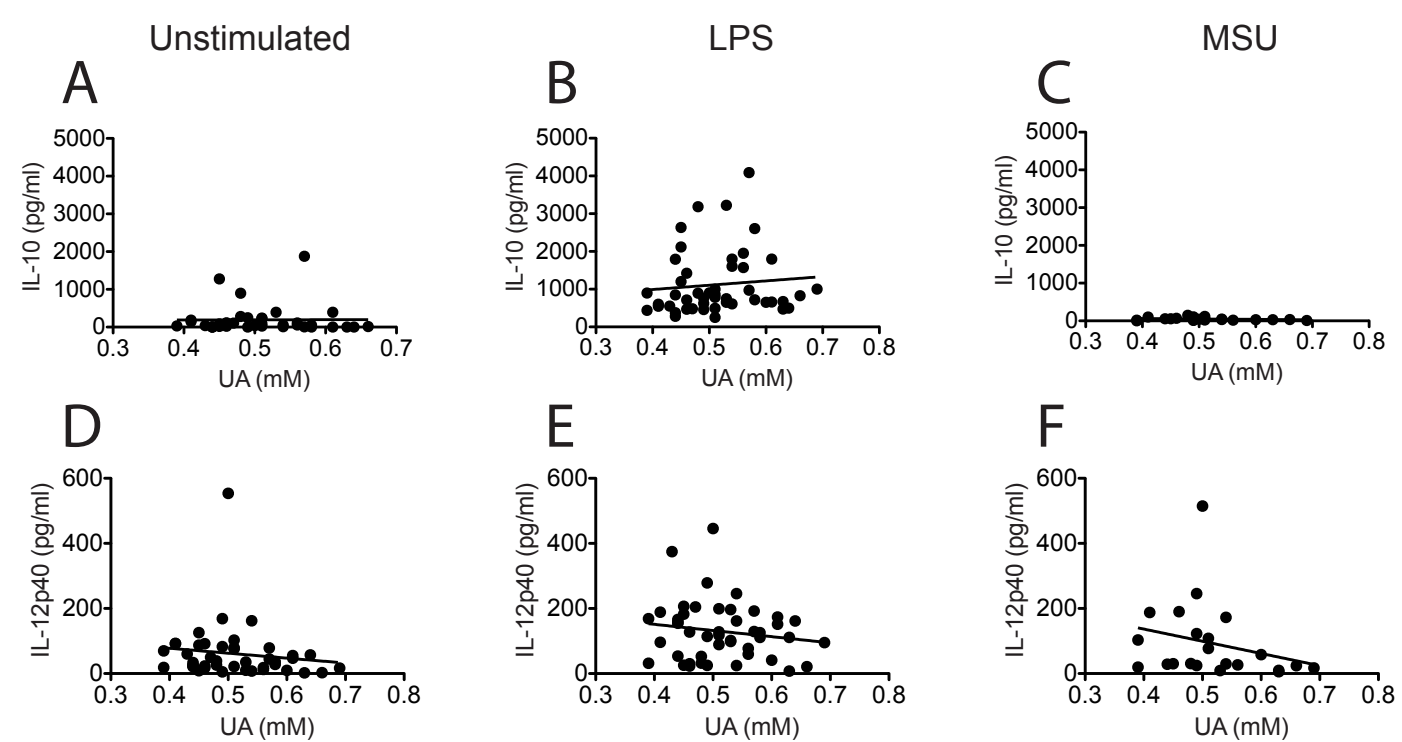

Figure 5.8 Correlation analysis of monocyte IL-10 and IL12p40 production versus serum UA levels.

Patient blood monocytes were isolated and cultured for $18 \mathrm{hr}$ unstimulated $(n=15)$ or with LPS $(100 \mathrm{ng} / \mathrm{ml} n=15)$ or $M S U(0.5 \mathrm{mg} / \mathrm{ml} n=7)$ stimulation. Data is from each patient at each time point compared to patient serum UA at each time point. A-C: IL10 and D-F: IL-12p40 in cell culture supernatants were analysed by ELISA or multiplex bead array.

ULT did not affect monocyte production of TNF $\alpha$ or IL-6 regardless of ex vivo stimulation (figure 5.9).

However, when TNF $\alpha$ and IL-6 production was analysed in relation to serum UA levels there was a significant negative correlation between serum UA and both TNF $\alpha$ and IL-6 from MSU-stimulated monocytes (figure 5.10c and f). 

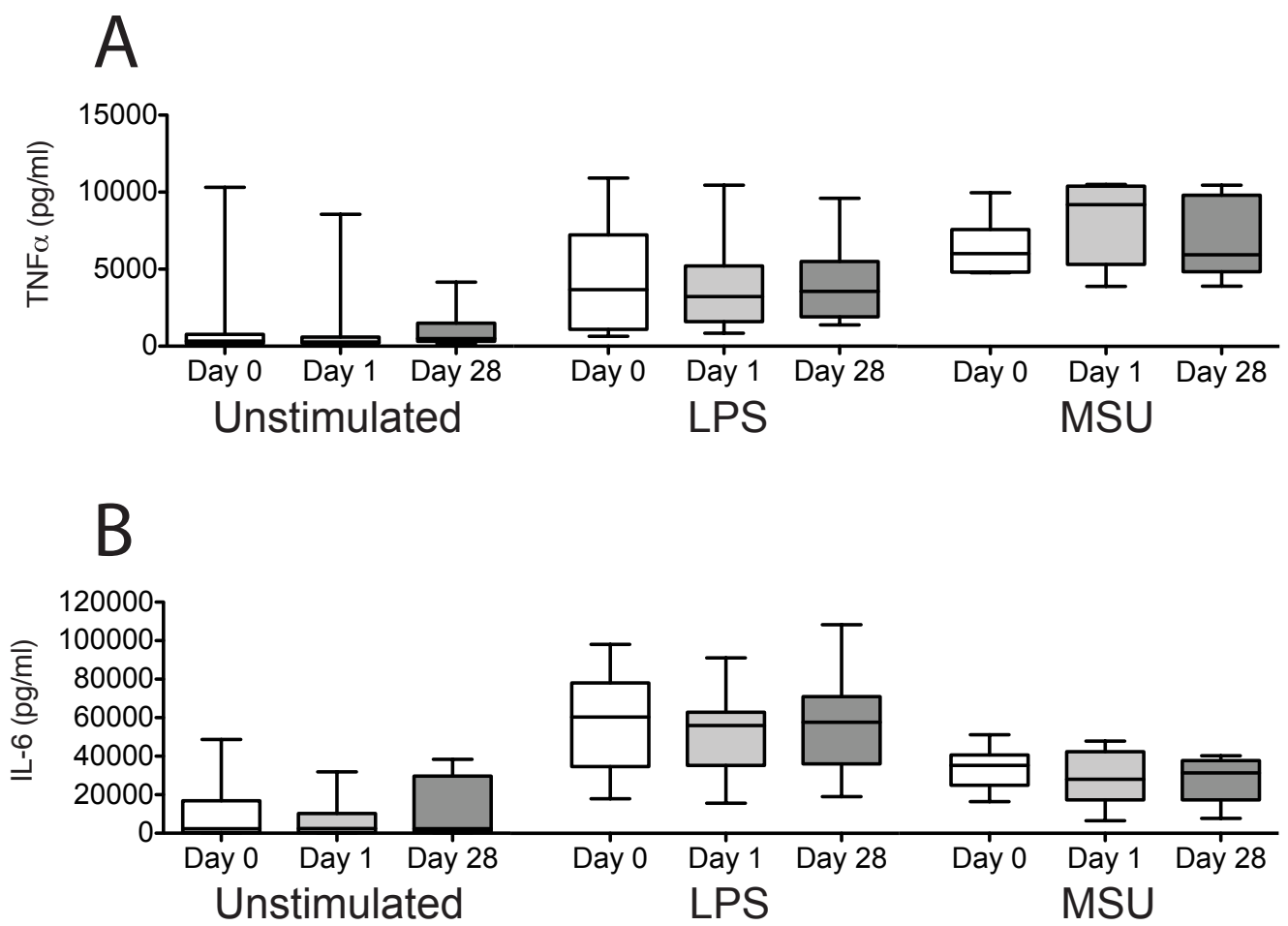

Figure 5.9 ULT does not affect monocyte TNF $\alpha$ or IL-6 production.

Patient blood monocytes were isolated and cultured for $18 \mathrm{hr}$ unstimulated $(n=15)$ or with LPS (100ng/ml $n=15)$ or MSU $(0.5 \mathrm{mg} / \mathrm{ml} n=7)$ stimulation. A: TNF $\alpha$ and B: IL-6 in cell culture supernatants were analysed by ELISA or multiplex bead array. Graphs display median, upper and lower quartiles, maximum and minimum.
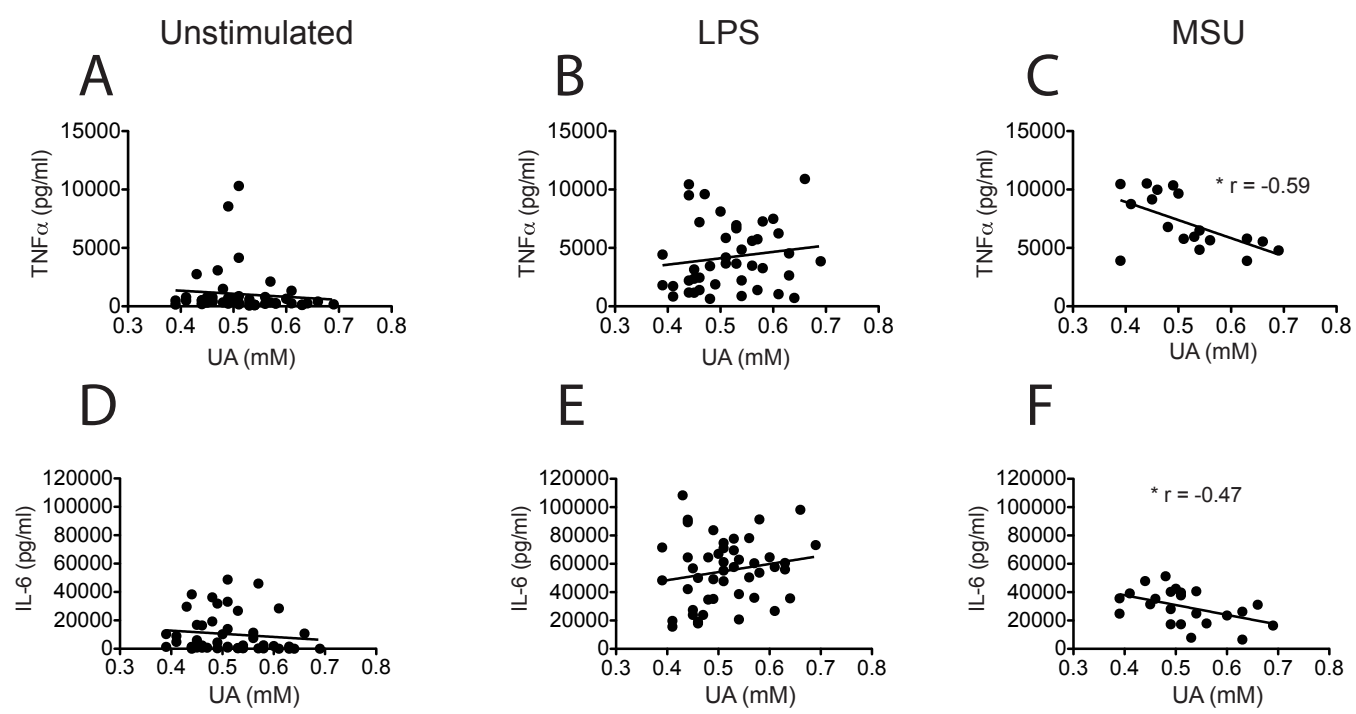

Figure 5.10 Serum UA negatively correlates with MSU-induced monocyte TNF $\alpha$ and IL-6 production.

Patient blood monocytes were isolated and cultured for $18 \mathrm{hr}$ unstimulated $(n=15)$ or with LPS $(100 \mathrm{ng} / \mathrm{ml} n=15)$ or $M S U(0.5 \mathrm{mg} / \mathrm{ml} n=7)$ stimulation. Data is from each patient at each time point compared to patient serum UA at each time point. A-C: TNF $\alpha$ and D-F: IL-6 in cell culture supernatants were analysed by ELISA or multiplex bead array. ${ }^{*} p<0.05$, Pearson's correlation coefficient. 
I demonstrated in Chapter 3 that in vitro UA treatment decreased IL- $\beta$ production in LPS-stimulated monocytes. IL-1 receptor antagonist (IL-1Ra) is produced as a negative feedback signal to inhibit IL-1 $\beta$ signalling ${ }^{199}$. Therefore, I measured the effect of ULT on both IL-1 $\beta$ and IL-1Ra in monocyte culture supernatants.

ULT had no effect on either unstimulated or LPS-induced monocyte IL-1 $\beta$ production but MSU-stimulated monocytes produced less IL-1 $\beta$ after 28 days of ULT compared to day 0 (figure 5.11a). ULT did not affect IL1-Ra production in either unstimulated or stimulated monocytes (figure 5.11b).

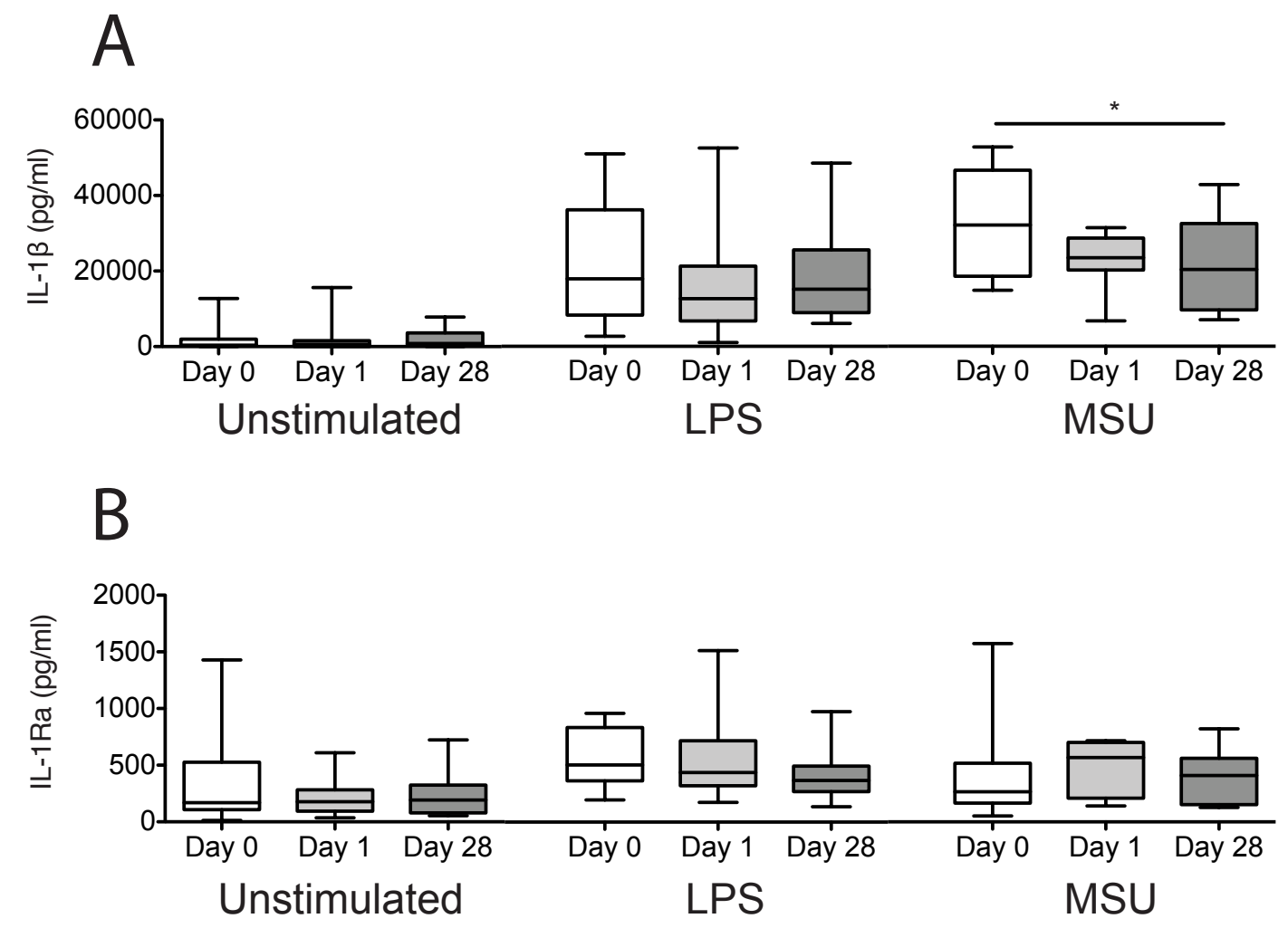

Figure 5.11 MSU-induced monocyte IL-1 $\beta$ production is reduced following ULT.

Patient blood monocytes were isolated and cultured for $18 \mathrm{hr}$ unstimulated ( $n=15)$ or with LPS $(100 \mathrm{ng} / \mathrm{ml} n=15)$ or MSU $(0.5 \mathrm{mg} / \mathrm{ml} n=7)$ stimulation. A: IL-1 $\beta$ and $B: I L-$ $1 R a$ in cell culture supernatants were analysed by ELISA or multiplex bead array. ${ }^{*} p<0.05$, paired samples $t$-test. Graphs display median, upper and lower quartiles, maximum and minimum. 
When monocyte IL-1 $\beta$ and IL1-Ra production was analysed in relation to serum UA levels there were no clear associations between serum UA levels and monocyte IL-1 $\beta$ or IL-1Ra production in either unstimulated or stimulated cells (figure 5.12).
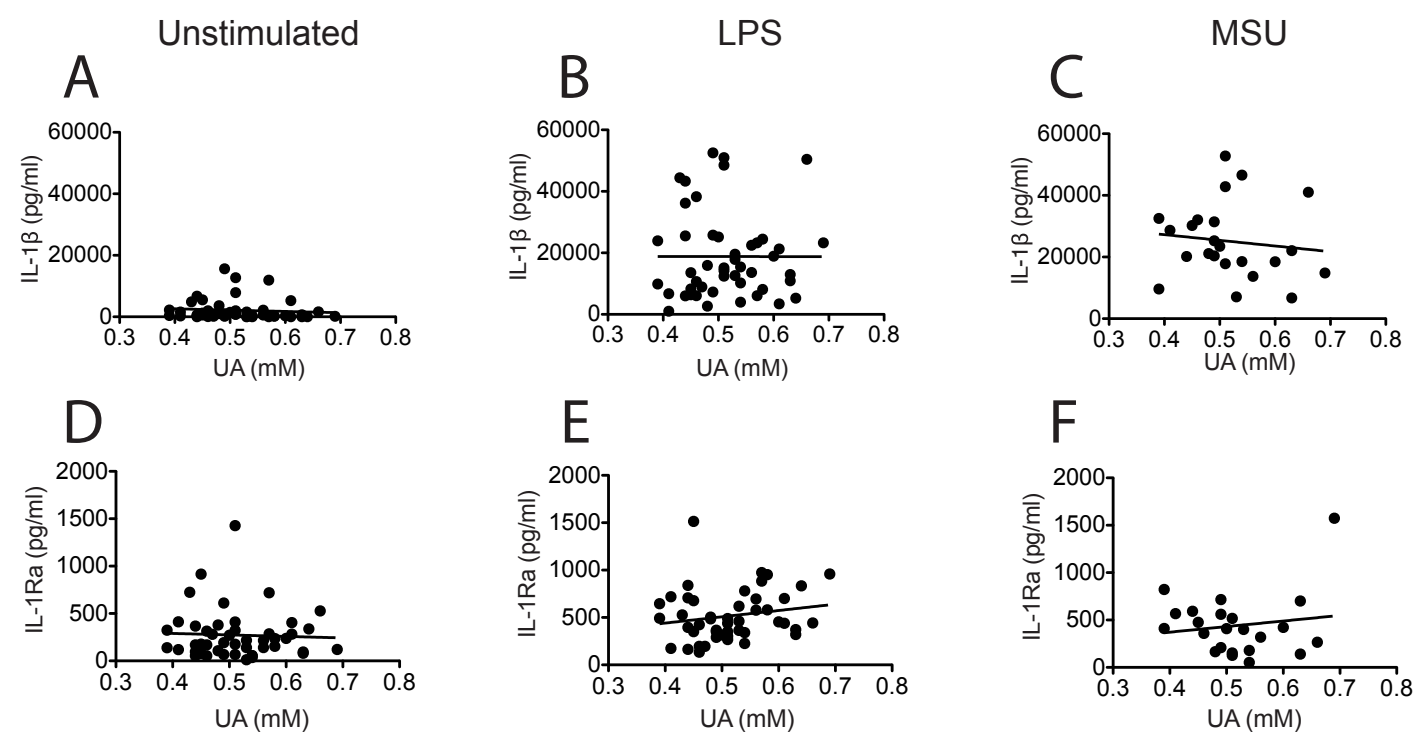

Figure 5.12 Correlation analysis of monocyte IL-1 $\beta$ and IL-1Ra production compared to serum UA.

Patient blood monocytes were isolated and cultured for $18 \mathrm{hr}$ unstimulated $(n=15)$ or with LPS $(100 \mathrm{ng} / \mathrm{ml} n=15)$ or MSU $(0.5 \mathrm{mg} / \mathrm{ml} n=7)$ stimulation. Data is from each patient at each time point compared to patient serum UA at each time point. A-C: IL$1 \beta$ and $D-F:$ IL-1Ra in cell culture supernatants were analysed by ELISA or multiplex bead array.

ULT reduced the production of IL-10 from monocytes stimulated with LPS and there was a negative correlation between TNF $\alpha$ and IL-6 production after MSU stimulation and serum UA levels. Taken together these results indicated that ULT was removing an inhibitory effect of soluble UA to garner a more inflammatory monocyte response to both LPS and MSU. However, ULT reduced monocyte production of $\mathrm{IL}-1 \beta$ following MSU stimulation, which suggests that UA may have differential effects on the mechanisms controlling IL-1 $\beta$ production and maturation compared to IL-10, TNF $\alpha$ and IL-6 following LPS vs. MSU stimulation. 


\subsubsection{Monocyte chemokine production following ULT}

Monocytes are an important source of chemokines during inflammation and chemokines released from the site of inflammation enhance recruitment of immune cells into the inflamed area. Therefore, I explored the effect of ULT on monocyte chemokine production. I measured the production of chemokines (IL-8, CCL2, CCL4, CCL5 and CCL7) in the culture supernatants of isolated monocytes stimulated with LPS or MSU for $18 \mathrm{hr}$.

There was no effect of ULT on monocyte IL-8 production in unstimulated or stimulated cells (figure 5.13a). There was a trend towards increased CCL2 production in untreated and LPS treated monocytes after ULT and a significant increase in CCL2 production in MSU-stimulated monocytes after 28 days of ULT compared to monocytes from day 0 (figure 5.13b).

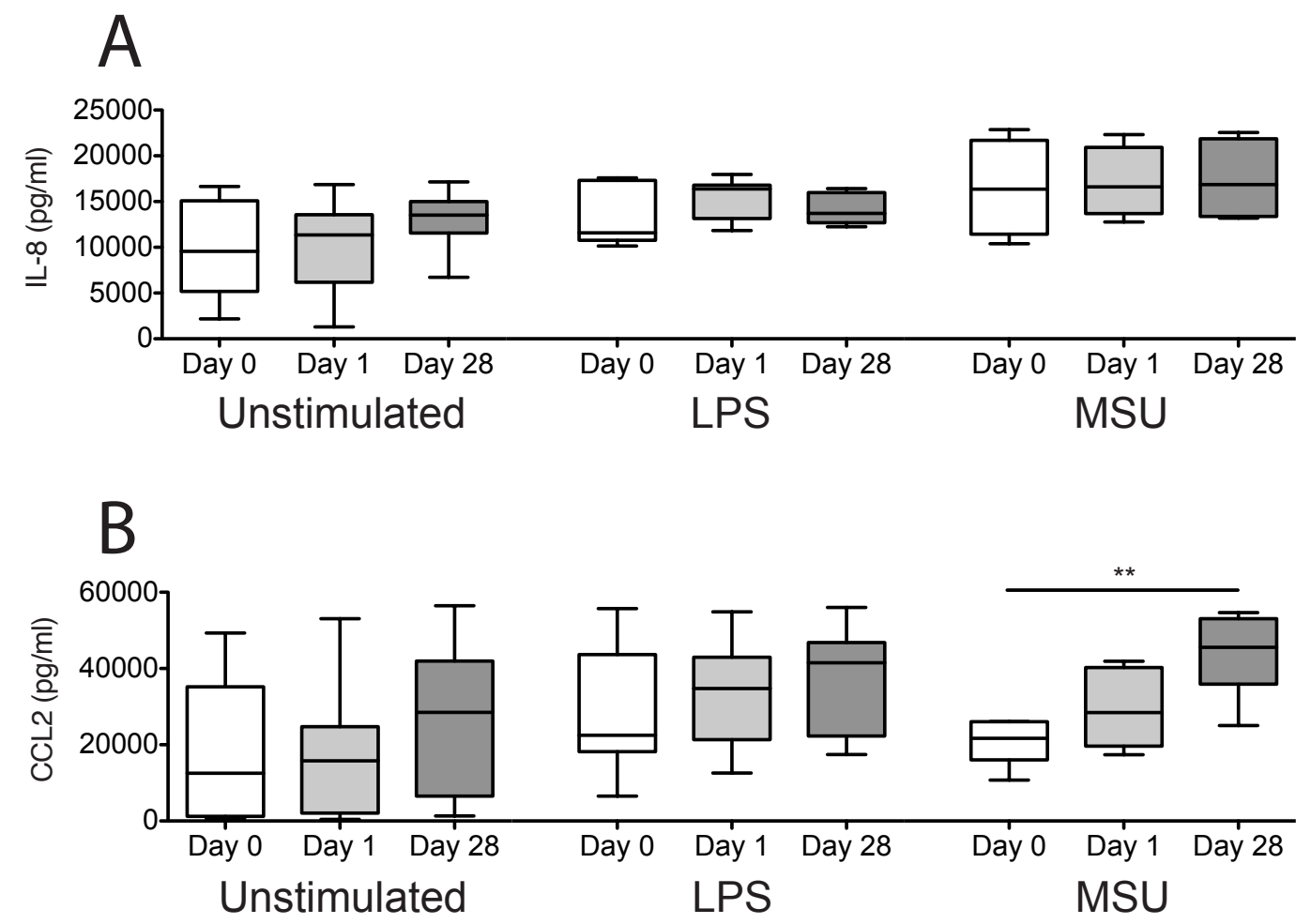

Figure 5.13 MSU-induced monocyte CCL2 production increases following ULT.

Patient blood monocytes were isolated and cultured for 18hr unstimulated $(n=15)$ or with LPS (100ng/m/ $n=15)$ or MSU $(0.5 \mathrm{mg} / \mathrm{ml} n=7)$ stimulation. A: IL-8 and B: CCL2 in cell culture supernatants were analysed by ELISA or multiplex bead array. ${ }^{* *} p<0.01$, paired samples $t$-test. Graphs display median, upper and lower quartiles, maximum and minimum. 
When monocyte chemokine production was analysed in relation to serum UA levels there were no clear associations between either IL-8 or CCL2 production and serum UA regardless of stimulation (figure 5.14).
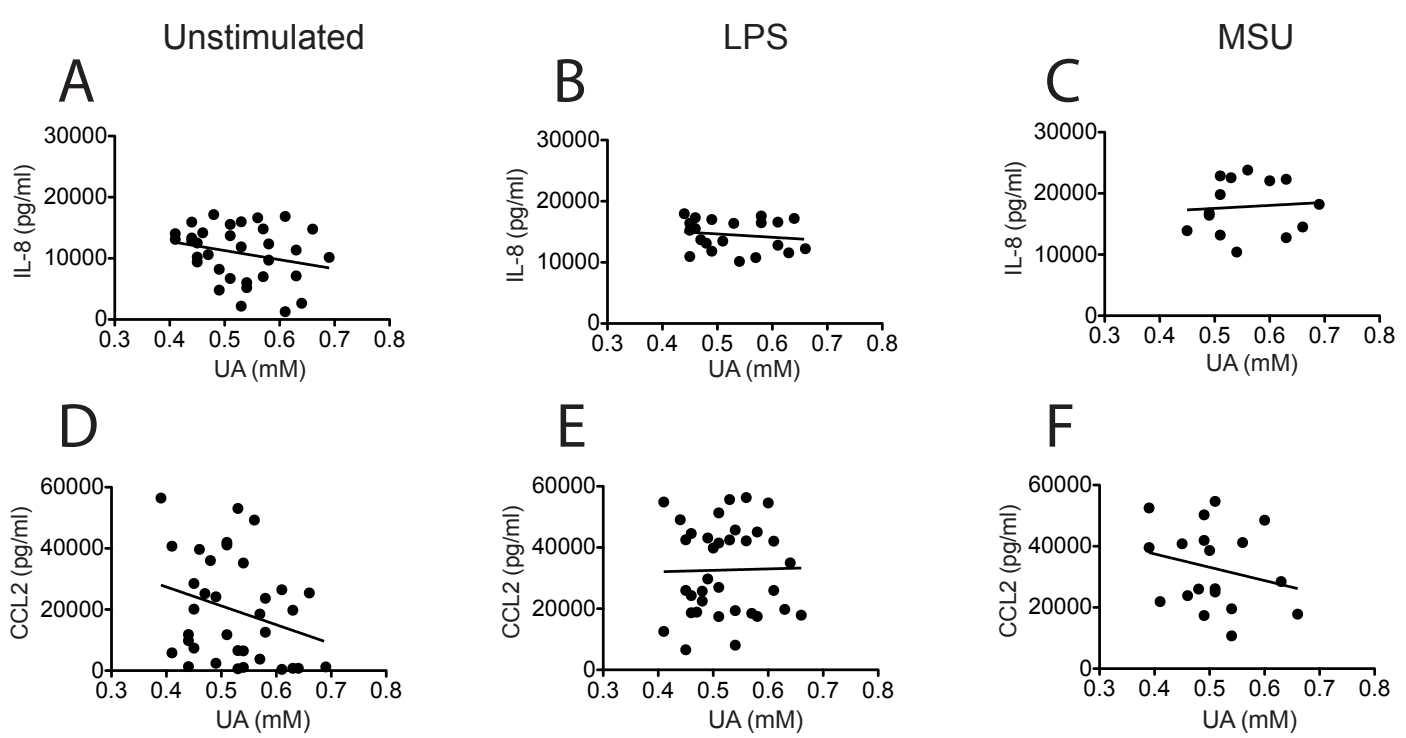

Figure 5.14 Correlation analysis of monocyte IL-8 and CCL2 production compared to serum UA.

Patient blood monocytes were isolated and cultured for $18 \mathrm{hr}$ unstimulated $(n=15)$ or with LPS $(100 \mathrm{ng} / \mathrm{ml} n=15)$ or $M S U(0.5 \mathrm{mg} / \mathrm{ml} n=7)$ stimulation. Data is from each patient at each time point compared to patient serum UA at each time point. A-C: IL8 and D-F: CCL2 in cell culture supernatants were analysed by ELISA or multiplex bead array.

I also measured CCL4, CCL5 and CCL7 to investigate the broader effect of ULT on monocyte chemokine production following LPS or MSU stimulation. ULT did not affect production of CCL4, CCL5 and CCL7 in unstimulated monocytes (figure 5.15, Unstimulated). There was a trend towards increased production of all three chemokines in LPS-stimulated monocytes (figure 5.15, LPS). In MSU-stimulated monocytes there was a trend towards increased production of CCL5 after ULT and a significant increase in CCL7 after both 1 and 28 days of ULT (figure $5.15 b-c, M S U$ ). 

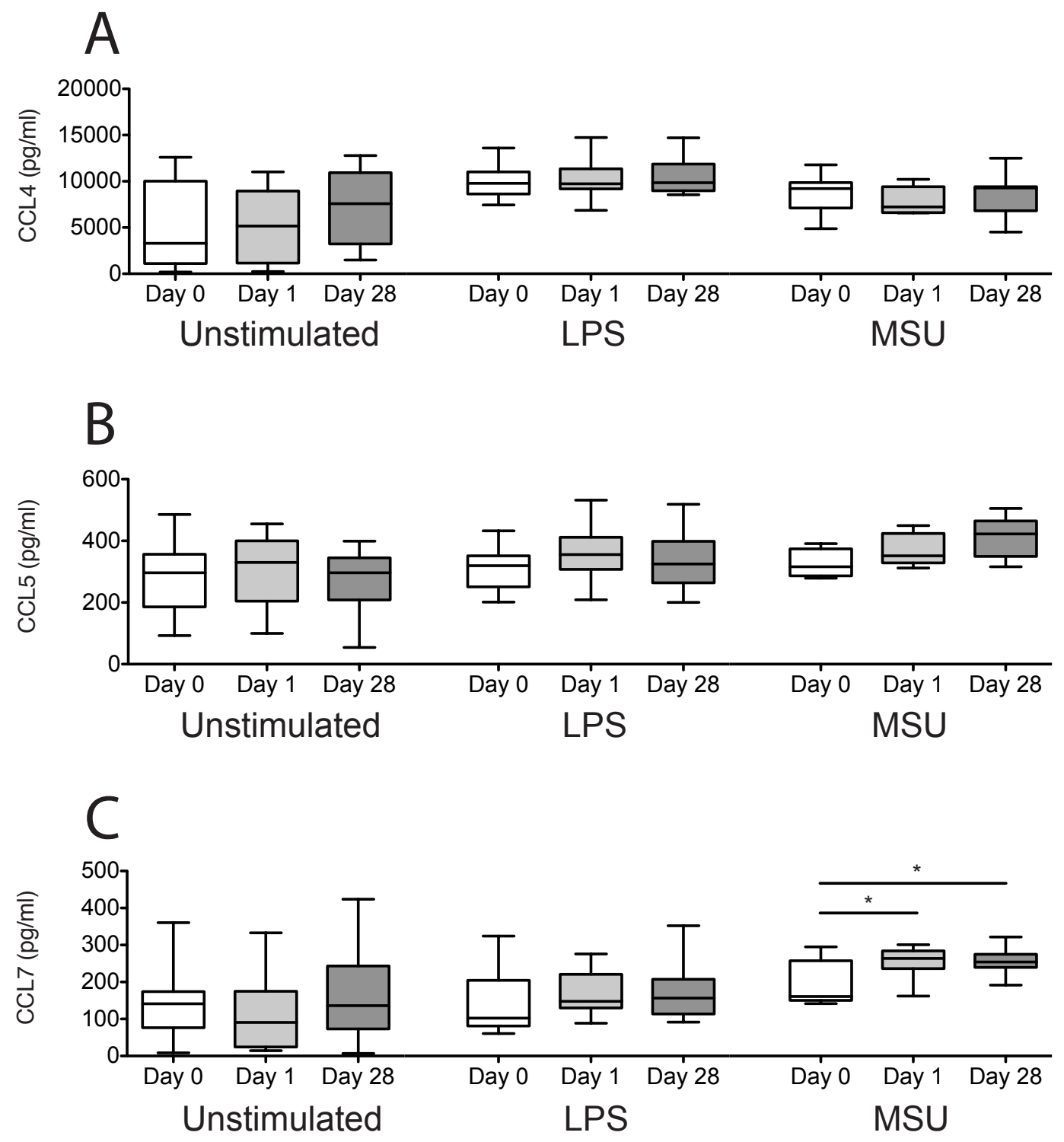

Figure 5.15 MSU-induced monocyte CCL7 production increases following ULT.

Patient blood monocytes were isolated and cultured for 18hr unstimulated $(n=15)$ or with LPS $(100 \mathrm{ng} / \mathrm{ml} n=15)$ or MSU $(0.5 \mathrm{mg} / \mathrm{ml} n=7)$ stimulation. A: CCL4, B: CCL5 and C: CCL7 in cell culture supernatants were analysed by ELISA or multiplex bead array. ${ }^{*} p<0.05$, paired samples $t$-test. Graphs display median, upper and lower quartiles, maximum and minimum. 
Correlation analysis of chemokine production relative to serum UA levels showed that CCL4, CCL5 and CCL7 from unstimulated monocytes trended down with increasing serum UA levels (figure 5.16a, d and g). Furthermore, there was a significant negative correlation between chemokine production and serum UA levels of CCL4 in LPS-stimulated monocytes and CCL5 in MSU-stimulated monocytes (figure 5.16b and f).

Taken together, these results indicated that ULT was enhancing the production of multiple chemokines in LPS and MSU-stimulated monocytes and suggested that serum UA levels might have a stronger influence on CCL2 (monocytes) over IL-8 (neutrophils) dependent recruitment.
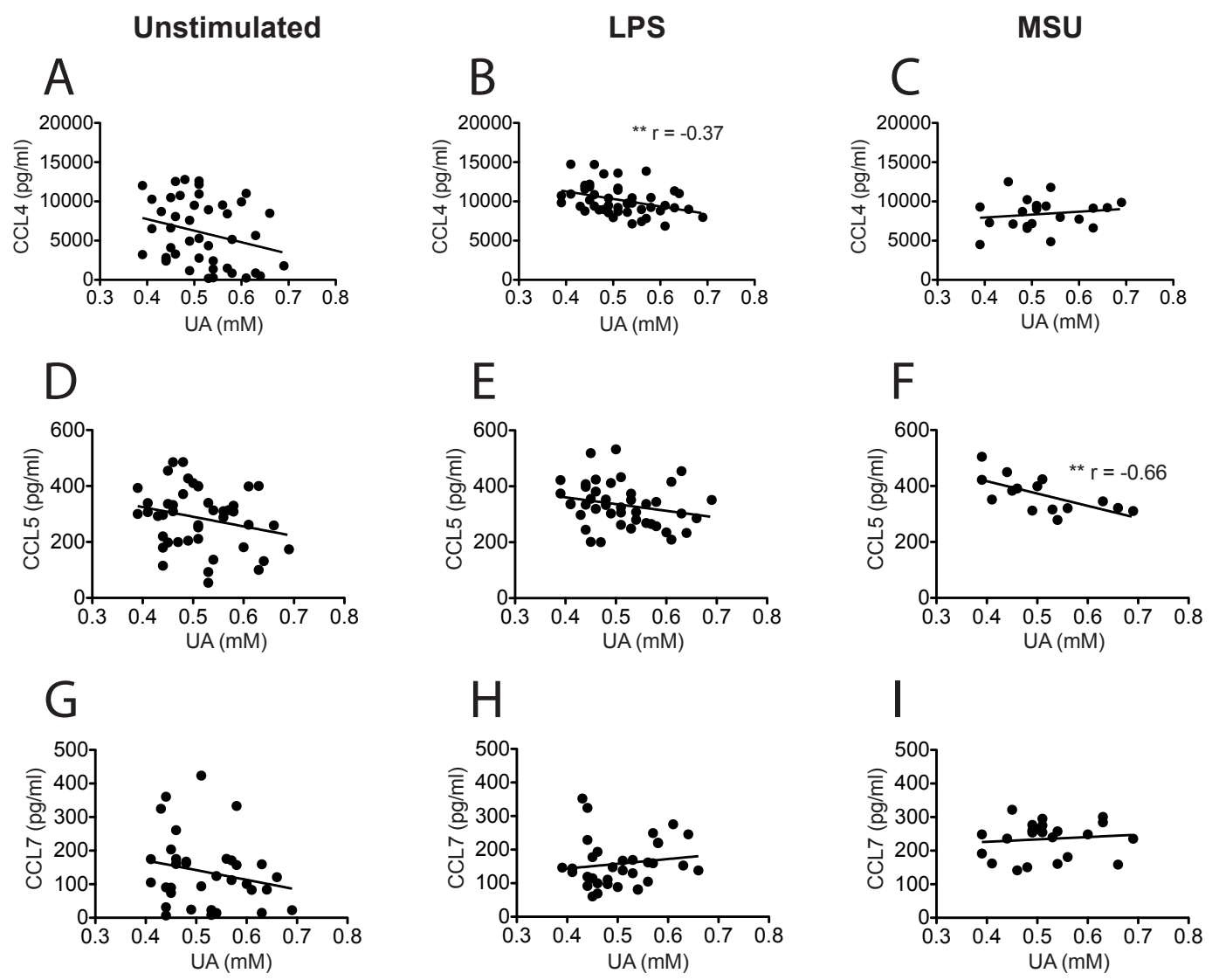

Figure 5.16 Correlation analysis of monocyte CCL4, CCL5 and CCL7 production compared to serum UA.

Patient blood monocytes were isolated and cultured for $18 \mathrm{hr}$ unstimulated $(n=15)$ or with LPS $(100 \mathrm{ng} / \mathrm{ml} n=15)$ or $M S U(0.5 \mathrm{mg} / \mathrm{ml} n=7)$ stimulation. Data is from each patient at each time point compared to patient serum UA at each time point. A-C: CCL4, D-F: CCL5 and G-I: CCL7 in cell culture supernatants were analysed by ELISA or multiplex bead array. ${ }^{* *} p<0.01$, Pearson's correlation coefficient. 


\subsubsection{ULT and cytosolic monocyte MAP kinase signalling}

In Chapter 3 and $4 \mathrm{I}$ showed that in vitro and in vivo hyperuricaemia suppressed inflammatory signalling in monocytes. Furthermore, the in vivo ULT studies suggested that some of these signalling changes could be reversed. Based on these results I hypothesised that ULT would increase monocyte inflammatory signalling in response to immune stimuli. In order to test this hypothesis I isolated cytosolic and nuclear protein from purified naive blood monocytes and from LPS or MSU-stimulated monocytes from patients at each stage of ULT and assessed the expression of inflammatory signalling molecules by western blotting.

Figure 5.17 is a representative western blot and the densitometry of the combined data is shown in figure 5.18. ULT increased phosphorylation of both p38 and ERK1/2 MAP kinases in unstimulated and LPS or MSU-stimulated cells. Importantly, ULT also increased cytosolic ASC expression in stimulated and unstimulated monocytes confirming $m y$ in vitro and in vivo results indicating that soluble UA levels control monocyte ASC expression (figure 5.17).

I used densitometry to combine the relative band intensities from each patient. The intensity of protein bands for each treatment day was measured using Image J software. These were normalised to evaluate the fold change in protein expression at Day 1 and Day 28 relative to Day 0 then normalised again to the corresponding density of $\beta$-actin to control for differences in protein loading. The blots used for this analysis can be found in appendices 7.12 and 7.13 .

Densitometric analysis of the combined western blots showed that ULT increased monocyte MAP kinase phosphorylation in unstimulated and LPS or MSU-stimulated cells (figure 5.18a-b). It also showed that 1 and 28 days of ULT increased monocyte ASC expression in unstimulated and stimulated monocytes (figure 5.18c). 


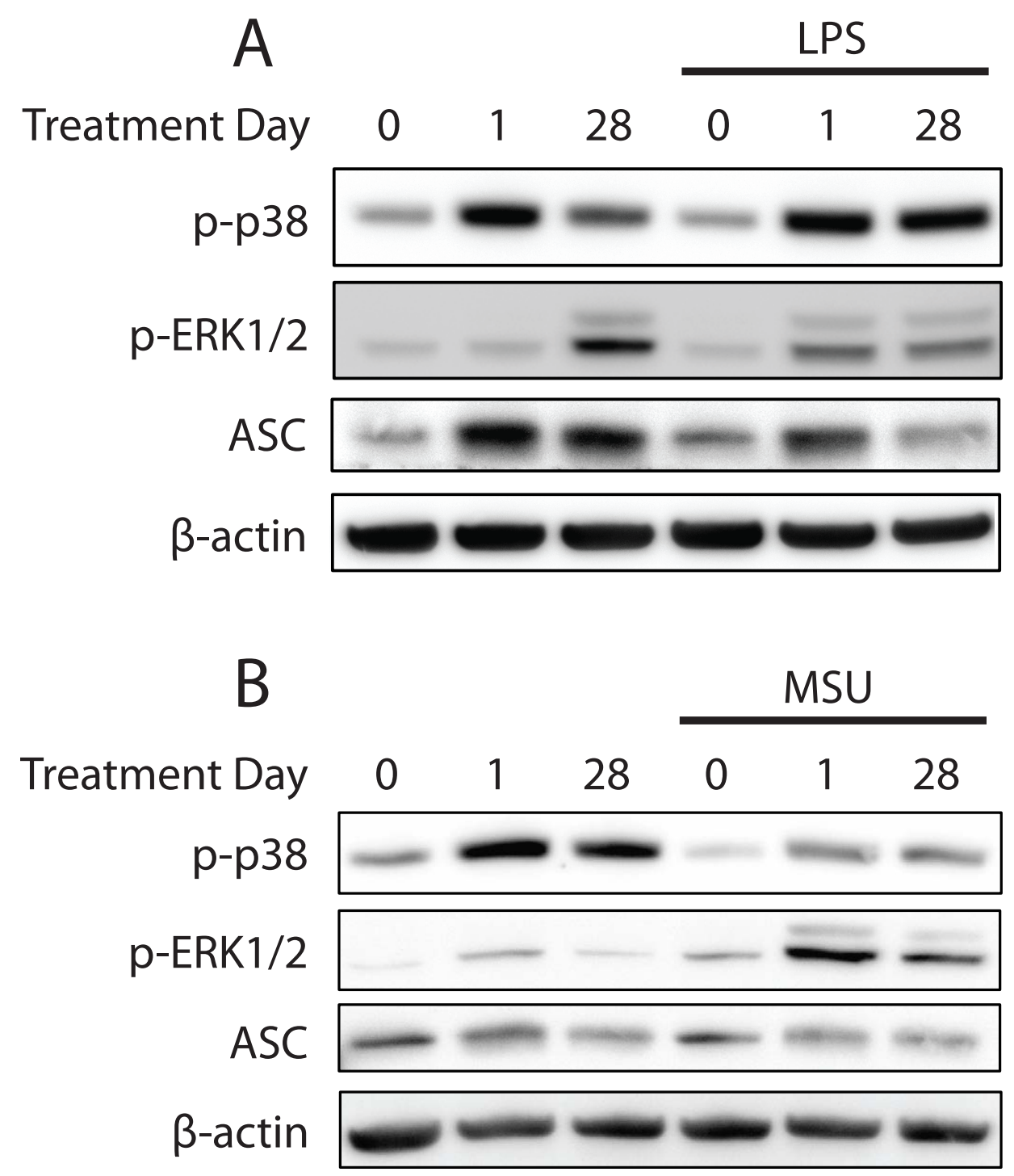

Figure 5.17 ULT increases monocyte MAP kinase phosphorylation and ASC expression.

Cytosolic protein was isolated from blood monocytes purified from patients before (0) and after 1 and 28 days of ULT. Monocytes were unstimulated $(n=8)$ or stimulated for $1 \mathrm{hr}$ with A: LPS $(100 \mathrm{ng} / \mathrm{ml} \mathrm{n}=4)$ or $B: M S U(0.5 \mathrm{mg} / \mathrm{ml} n=4)$. Protein extracts were analysed by western blotting. 


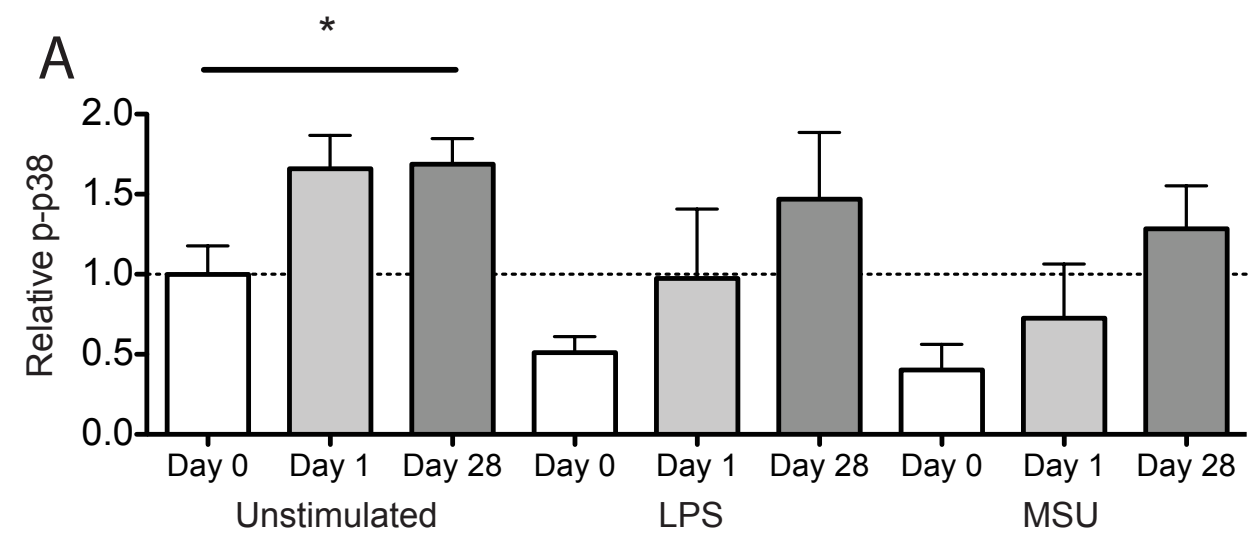

B
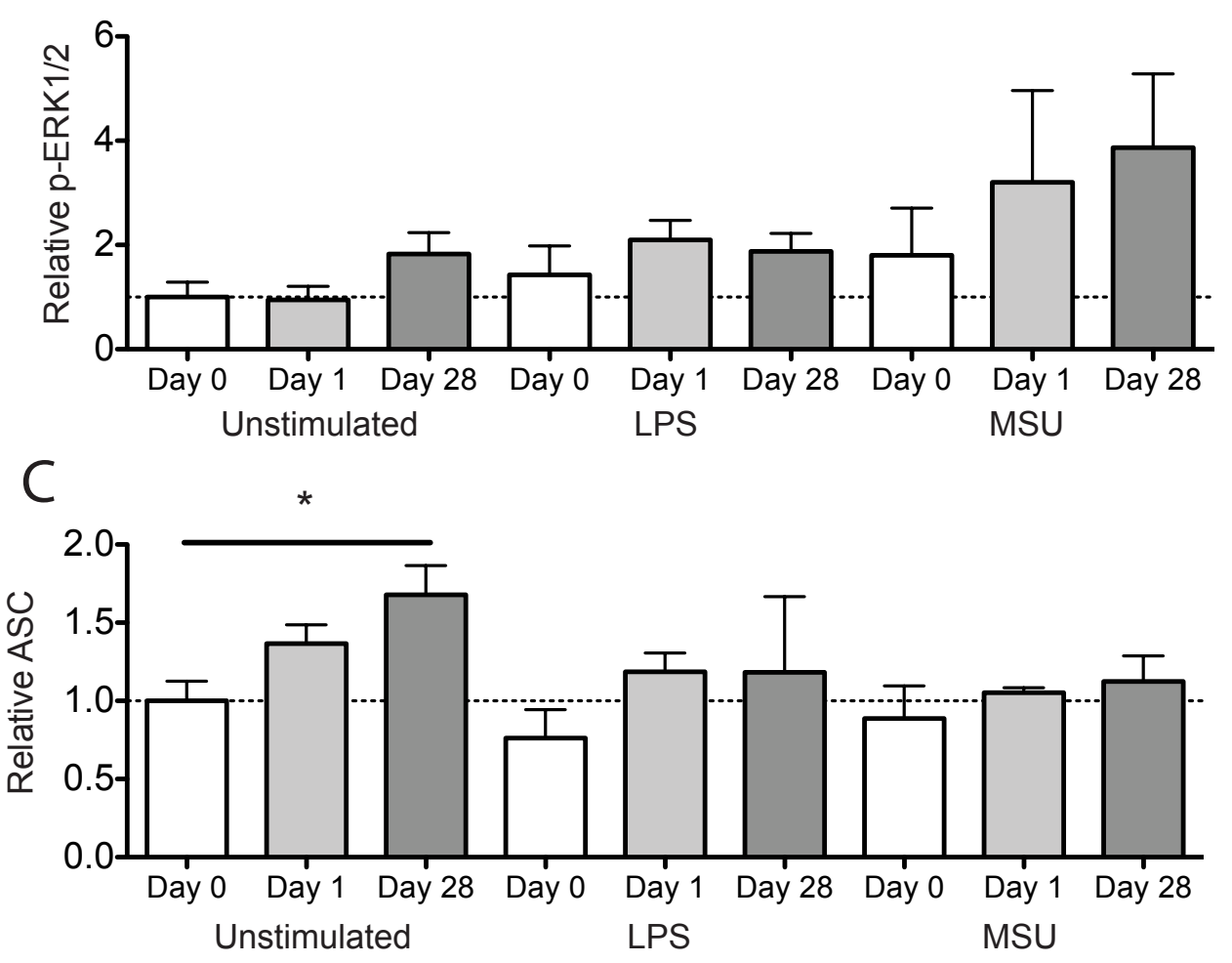

Figure 5.18 ULT increases monocyte MAP kinase phosphorylation and ASC expression.

Cytosolic protein was isolated from blood monocytes purified from patients before (0) and after 1 and 28 days of ULT. Monocytes were unstimulated $(n=8)$ or stimulated for $1 \mathrm{hr}$ with $A$ : LPS $(100 \mathrm{ng} / \mathrm{ml} \mathrm{n=4})$ or B: MSU $(0.5 \mathrm{mg} / \mathrm{ml} n=4)$. A: $p$-p38, B: $p$-ERK1/2 and $C$ : ASC western blot band intensity relative to $\beta$-actin and Day 0 were analysed using ImageJ. ${ }^{*} p<0.05$, $t$-test. Data are mean with SEM. 
DUSP10 and DUSP1 are regulators of MAP kinase phosphorylation that have been implicated in the control of IL-10 production ${ }^{173,200}$.

Figure 5.19 is a representative western blot of monocyte DUSP10 and DUSP1 and the phosphorylation status of DUSP1 and the densitometry of the combined data is shown in figure 5.20. ULT had no effect on the levels of DUSP10 or DUSP1 in unstimulated or stimulated monocytes (figure 5.19). However, ULT resulted in a large increase in DUSP1 phosphorylation in both LPS and MSU-stimulated monocytes (figure 5.19).

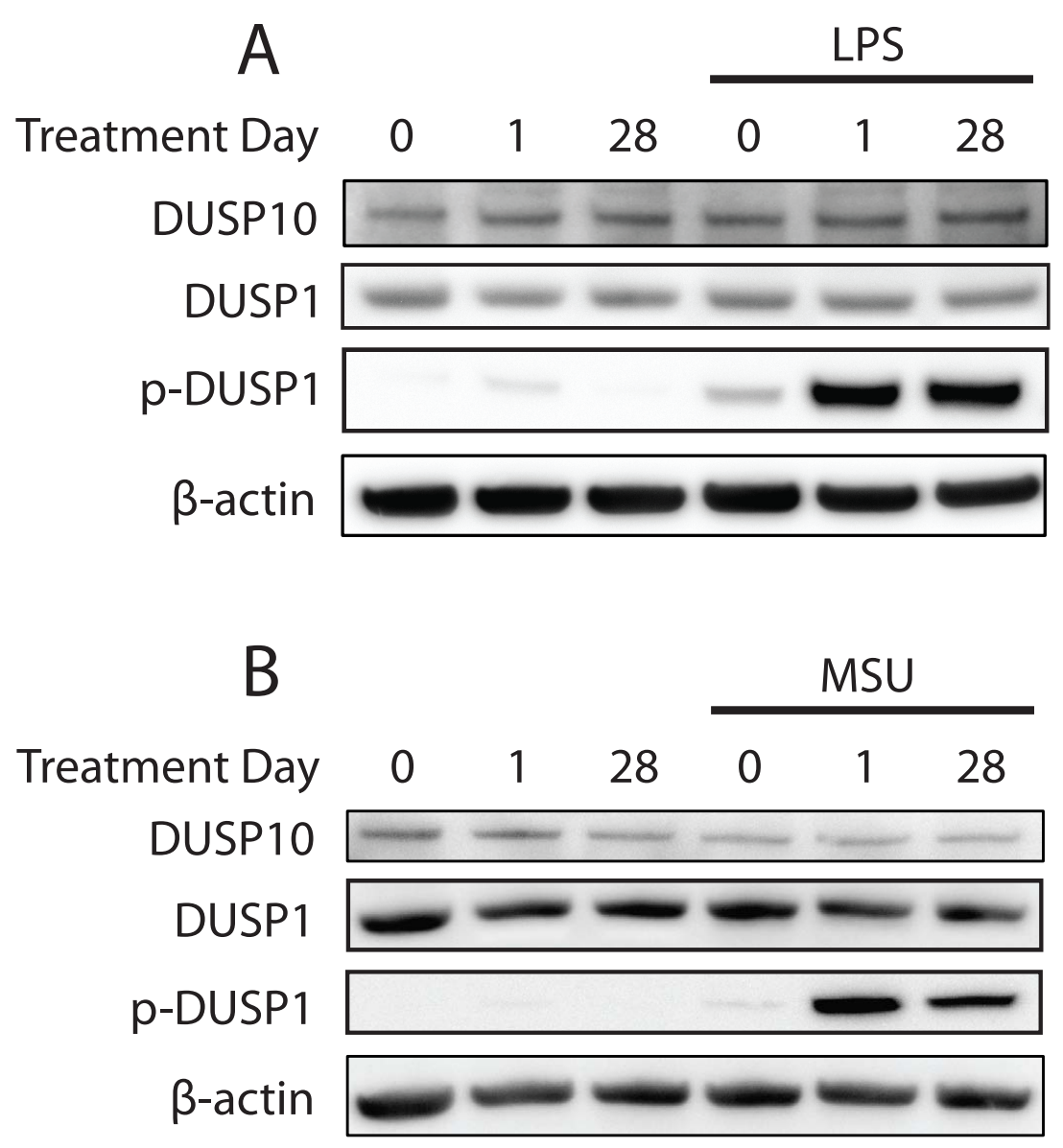

Figure 5.19 ULT increases monocyte DUSP1 phosphorylation.

Cytosolic protein was isolated from blood monocytes purified from patients before (0) and after 1 and 28 days of ULT. Monocytes were unstimulated $(n=8)$ or stimulated for $1 \mathrm{hr}$ with A: LPS $(100 \mathrm{ng} / \mathrm{ml} \mathrm{n}=4)$ or $B: \mathrm{MSU}(0.5 \mathrm{mg} / \mathrm{ml} \mathrm{n}=4)$. Protein extracts were analysed by western blotting. 
When combined using densitometry the DUSP10, DUSP1 and p-DUSP1 western blots showed that ULT had no effect on the expression levels of DUSP10 and DUSP1 (figure 5.20a-b). However, after ULT there was an average 10-fold and 15-fold higher level of phosphorylated DUSP1 following LPS or MSU stimulation respectively (figure $5.20 \mathrm{c}$ ).

A

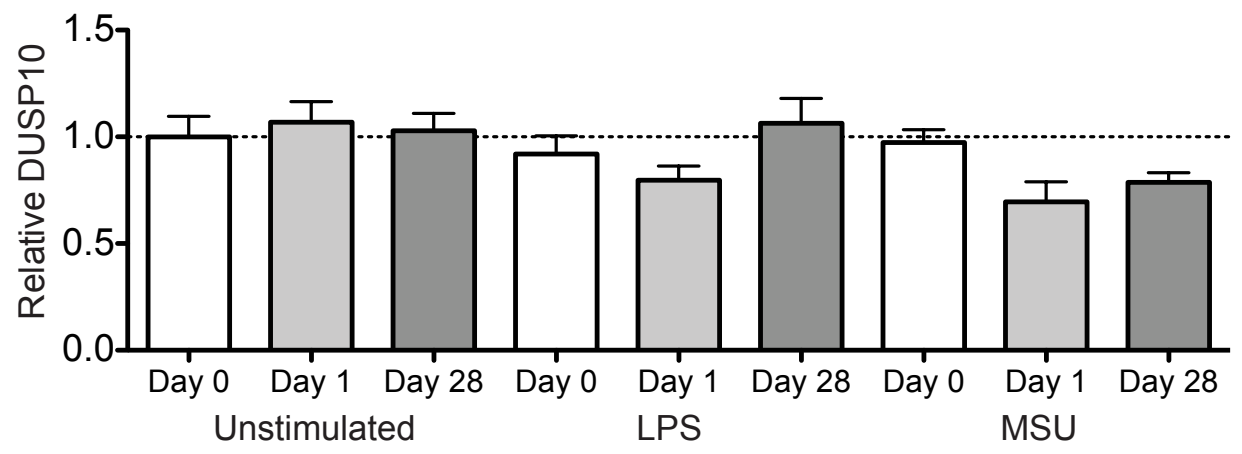

B

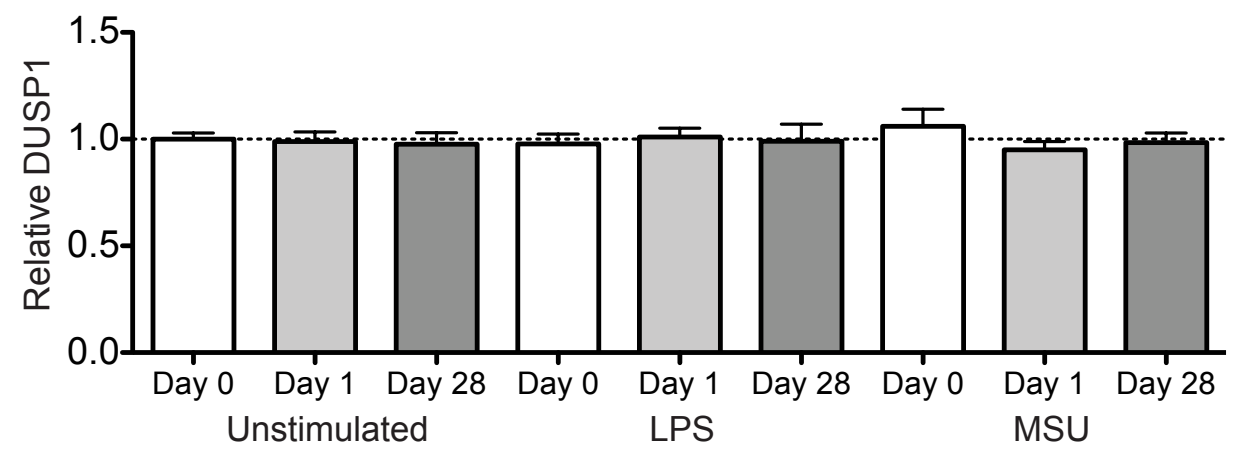

C

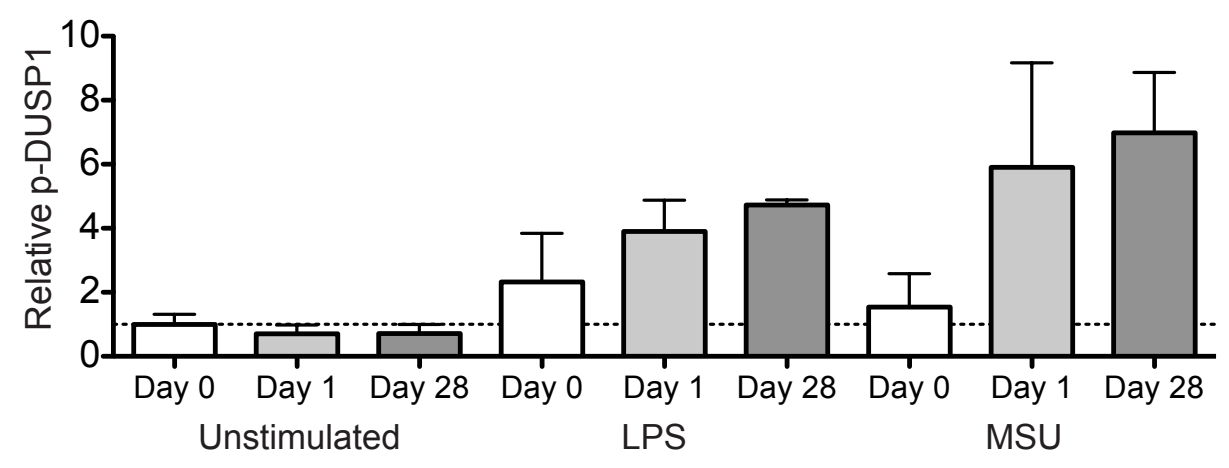

Figure 5.20 ULT increases monocyte DUSP1 phosphorylation.

Cytosolic protein was isolated from blood monocytes purified from patients before (0) and after 1 and 28 days of ULT. Monocytes were unstimulated $(n=8)$ or stimulated for 1hr with A: LPS $(100 \mathrm{ng} / \mathrm{ml} n=4)$ or B: MSU $(0.5 \mathrm{mg} / \mathrm{ml} n=4)$. A: DUSP10, B: DUSP1 and C: $p$-DUSP1 western blot band intensity relative to $\beta$-actin and Day 0 were analysed using ImageJ. Data are mean with SEM. 


\subsubsection{ULT and monocyte protein expression in the nucleus}

In Chapter 4 I showed that acute hyperuricaemia reduced the translocation of the NFкB p65 subunit into the nucleus of murine splenic monocytes following in vivo LPS stimulation. Additionally, ULT with allopurinol reversed this effect. Therefore, I wanted to establish whether ULT in patients with hyperuricaemia altered nuclear translocation of the p65 and p50 NFkB subunits in human blood monocytes.

Figure 5.21 is a representative western blot of nuclear extracts from patient blood monocytes and the densitometry of the combined data is shown in figure 5.22. ULT increased p65 nuclear translocation in monocytes stimulated with LPS but not MSU (figure 5.21 and figure 5.22a). ULT also increased p50 translocation in LPS stimulated monocytes (figure 5.22b).

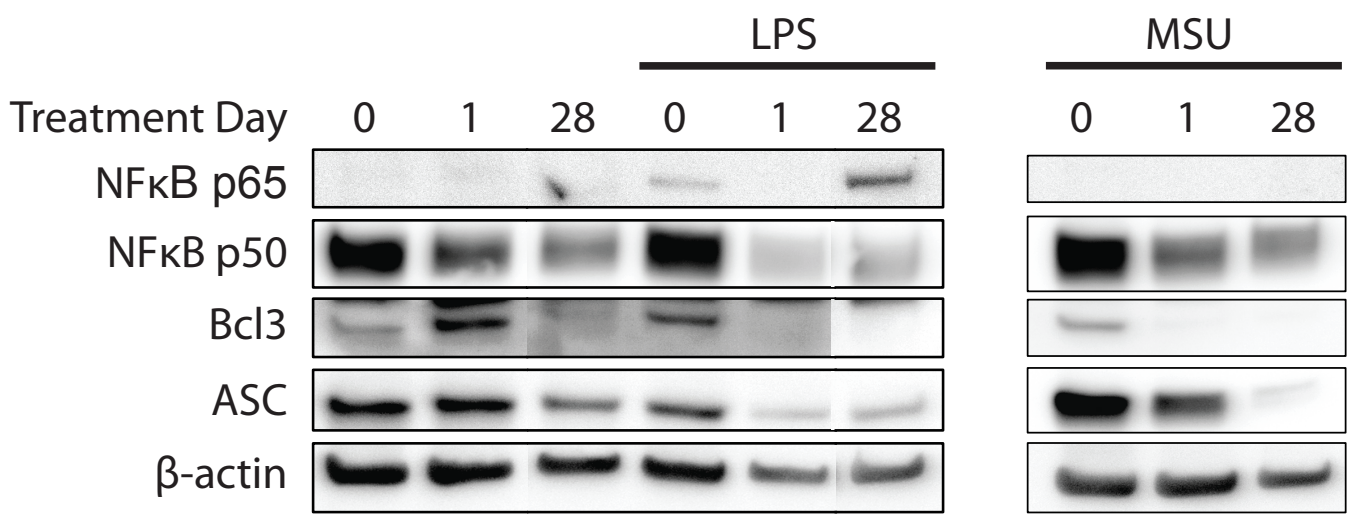

Figure 5.21 ULT increases monocyte NFKB p65 nuclear translocation and decreases nuclear p50.

Nuclear protein was isolated from blood monocytes purified from patients before (0) and after 1 and 28 days of ULT. Monocytes were unstimulated $(n=7)$ or stimulated for $1 \mathrm{hr}$ with A: LPS $(100 \mathrm{ng} / \mathrm{ml} \mathrm{n}=4)$ or $B:$ MSU $(0.5 \mathrm{mg} / \mathrm{ml} n=3)$. Protein extracts were analysed by western blotting. 


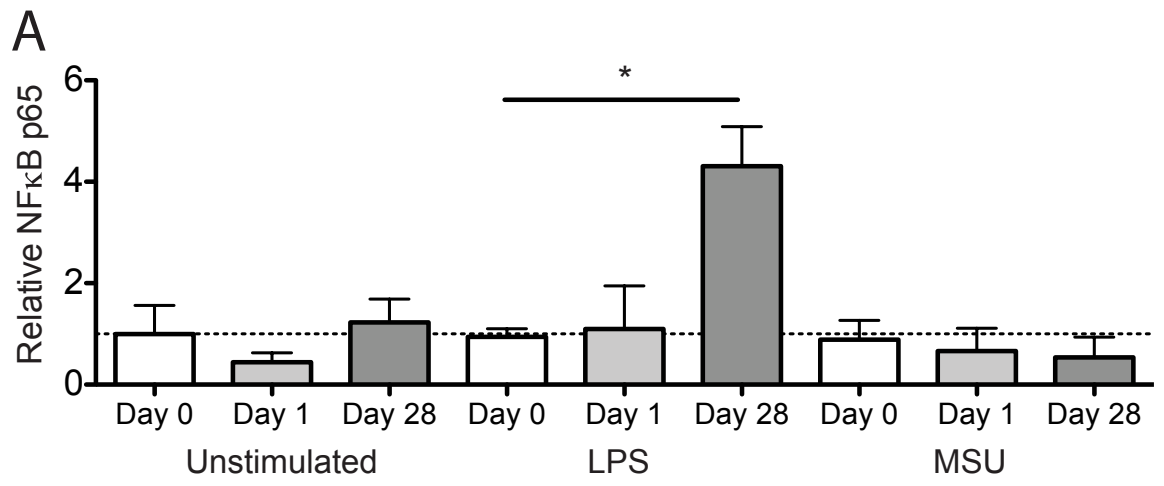

B

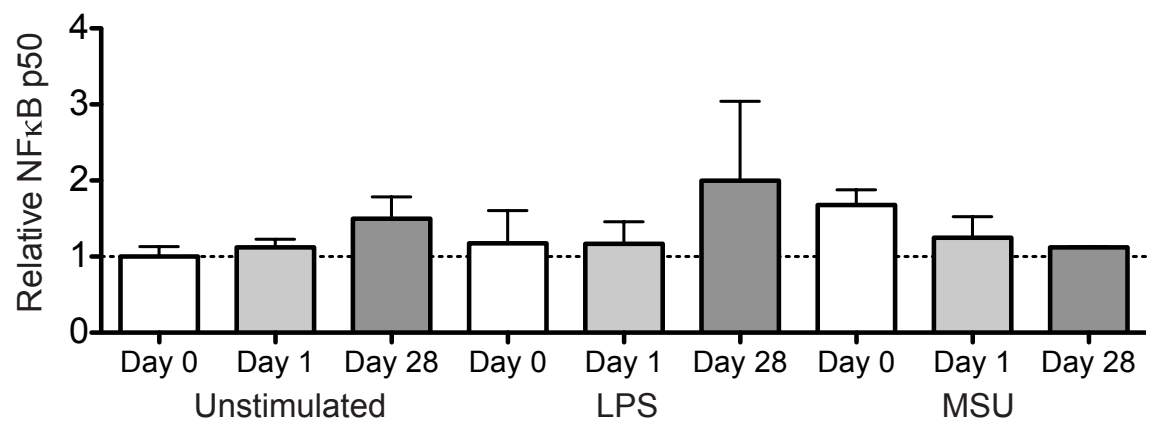

C

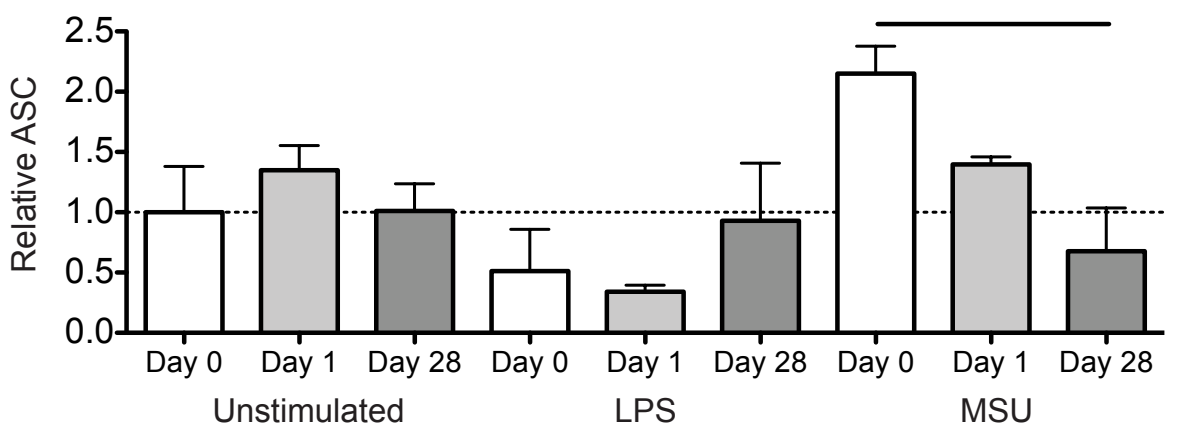

D

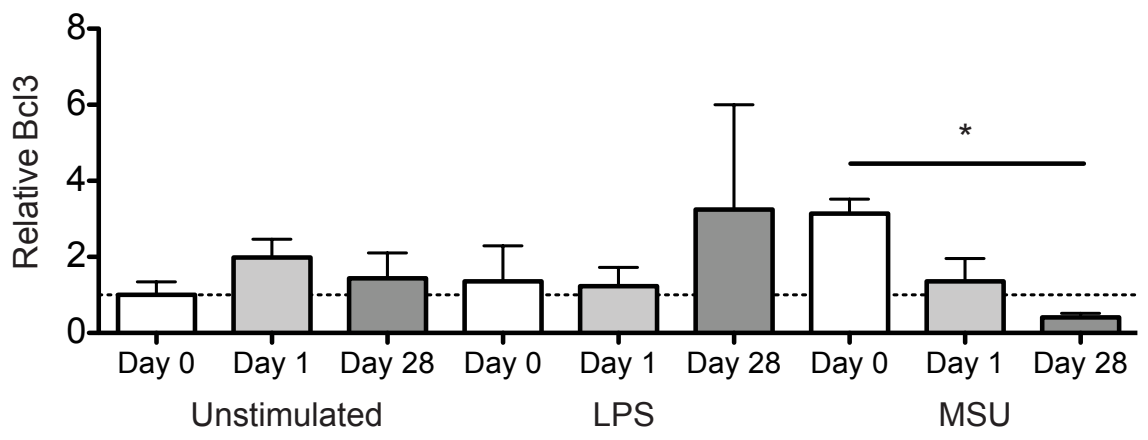

Figure 5.22 ULT increases NFKB nuclear translocation in LPS-stimulated monocytes but decreases ASC and Bc/3 in MSU-stimulated monocytes.

Nuclear protein was isolated from blood monocytes purified from patients before (0) and after 1 and 28 days of ULT. Monocytes were unstimulated $(n=7)$ or stimulated for $1 \mathrm{hr}$ with $A$ : LPS (100ng/ml n=4) or B: MSU $(0.5 \mathrm{mg} / \mathrm{ml} \mathrm{n=3})$. A: NFKB p65, B: NFKB p50, C: ASC and D: Bcl3 western blot band intensity relative to $\beta$-actin and Day 0 were analysed using ImageJ. ${ }^{*} p<0.05$, $t$-test. Data are mean with SEM. 
$\mathrm{Bcl} 3$ is an $1 \kappa \mathrm{B}$ protein that associates with the p50/p50 homodimer and has been reported to inhibit IL-10 transcription ${ }^{201}$. I measured nuclear $\mathrm{Bcl} 3$ expression to investigate the mechanism behind the control of p50 subunit nuclear translocation by soluble UA.

ASC has immunoregulatory effects in the nucleus by increasing mRNA stability in DC to support immune function ${ }^{176}$. Therefore, I wanted to investigate whether ULT altered nuclear expression of ASC in human blood monocytes.

Densitometric analysis of the combined western blots showed that ULT increased ASC and Bcl3 at day 1 in unstimulated monocytes and at day 28 in LPS-stimulated monocytes. However, both ASC and $\mathrm{Bcl} 3$ were reduced 1 and 28 days after ULT in MSU-stimulated monocytes (figure 5.22C and D).

The increased NFKB p65 and decreased p50 translocation following ULT showed that the inflammatory potential of human blood monocytes was increased following ULT.

\subsubsection{Monocyte inflammasome signalling following ULT}

In Chapter 4 I showed that in vivo ULT increased monocyte ASC expression and decreased expression of other NLRP3 inflammasome components. To investigate this effect in the context of ULT I measured monocyte expression of NLRP3 inflammasome components by western blotting.

Figure 5.23 is a representative western blot of monocyte NLRP3, caspase1 and IL-1 $\beta$ before and after ULT and the densitometry of the combined data is shown in figure 5.24. Expression of NLRP3 increased in unstimulated monocytes and LPS or MSU-stimulated monocytes after 1 and 28 days of ULT (figure 5.23). ULT increased pro-caspase1 expression and this corresponded with a decrease in caspase1. Monocyte pro-IL-1 $\beta$ expression increased after ULT in LPS and MSU treated cells. There was very little IL-1 $\beta$ expression in monocytes following ULT (figure 5.23a) and figure 5.23b shows that ULT decreased IL-1 $\beta$ expression with or without MSU stimulation. 

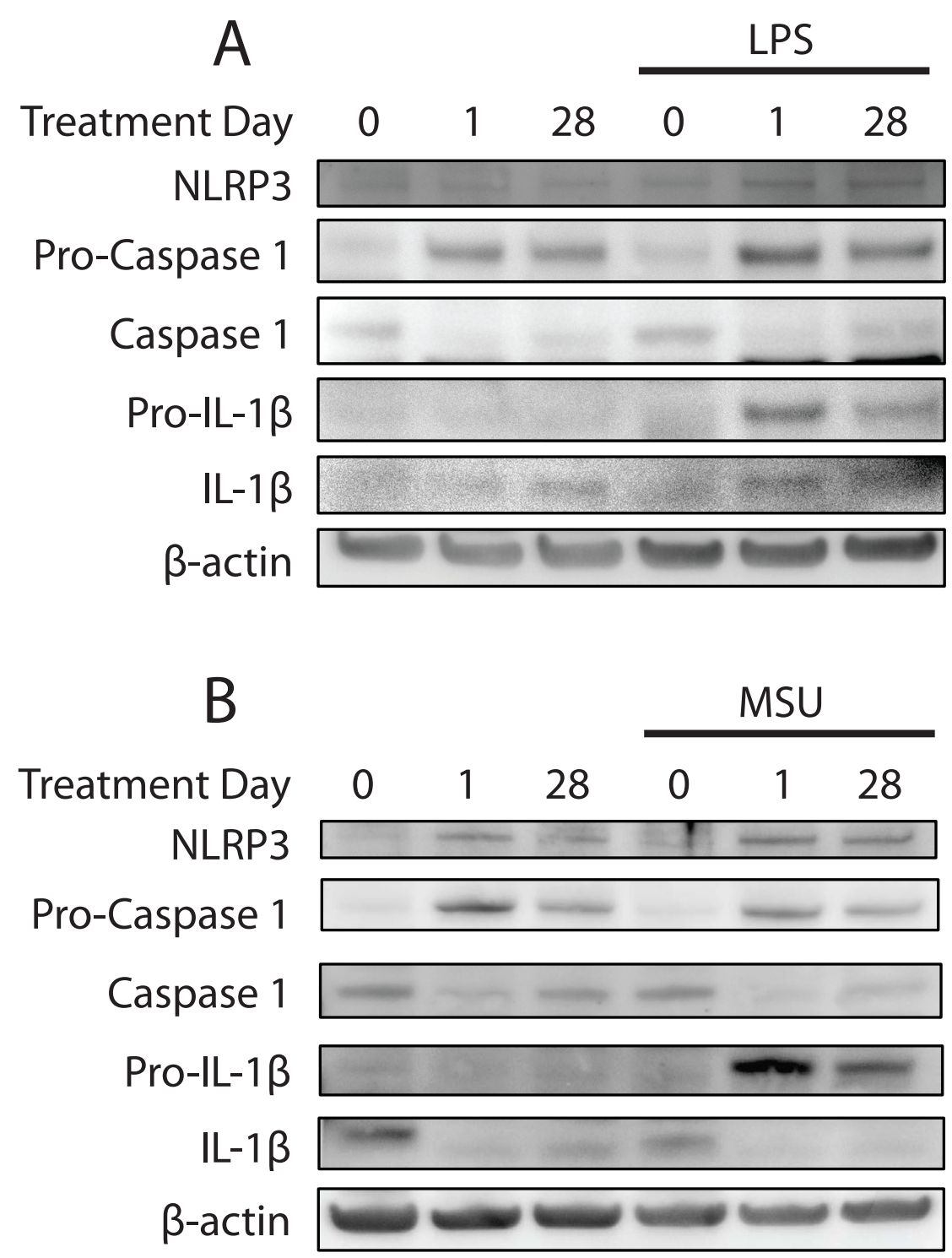

Figure 5.23 ULT increases monocyte NLRP3 inflammasome components.

Cytosolic protein was isolated from blood monocytes purified from patients before (0) and after 1 and 28 days of ULT. Monocytes were unstimulated $(n=8)$ or stimulated for $1 \mathrm{hr}$ with A: LPS $(100 \mathrm{ng} / \mathrm{ml} \mathrm{n=4})$ or $B:$ MSU $(0.5 \mathrm{mg} / \mathrm{ml} n=4)$. Protein extracts were analysed by western blotting. 
When the NLRP3 inflammasome western blots were normalised and combined using densitometry it shows that ULT resulted in a 3 to 5 -fold increase in NLRP3 and a 2 to 4 -fold increase in pro-caspase 1 expression in unstimulated and stimulated monocytes (figure 5.24a-b). Caspase1 expression had decreased at day 1 and decreased further by day 28 of ULT in unstimulated and stimulated monocytes (figure5.24c). Pro-IL-1 $\beta$ expression increased in LPS and MSU-stimulated monocytes following ULT (figure 5.24d). Contrary to the IL- $\beta$ expression in figure $5.23 \mathrm{~b}$, combined results showed that there was an overall increase in IL-1 $\beta$ expression following ULT in unstimulated monocytes but no difference in IL-1 $\beta$ expression after LPS or MSU stimulation (figure $5.24 \mathrm{e}$ ).

Overall ULT resulted in increased expression of NLRP3, pro-caspase1 and ASC, the three major subunits of the NLRP3 inflammasome and further confirms the inflammatory priming of human blood monocytes by ULT. 

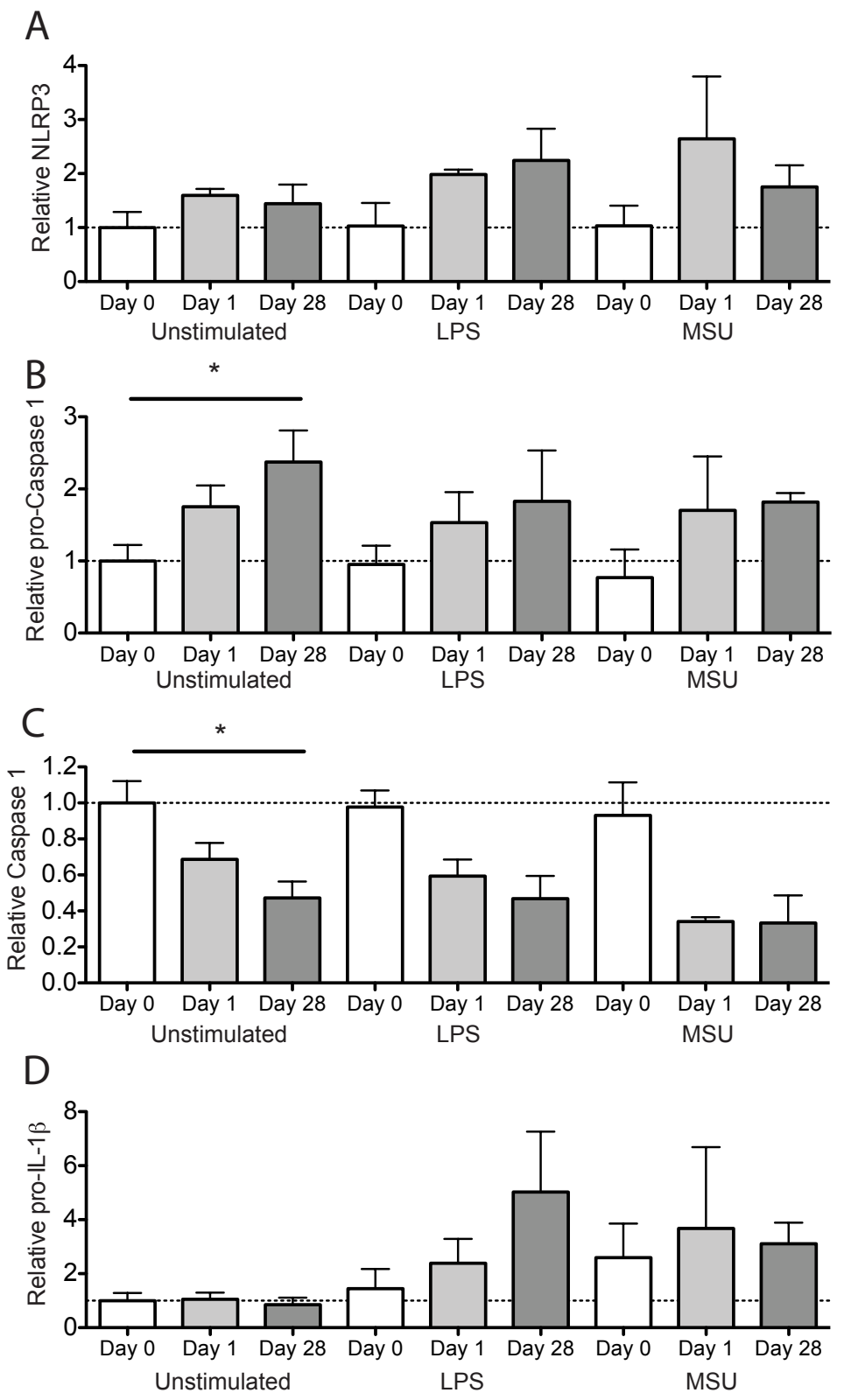

$E$

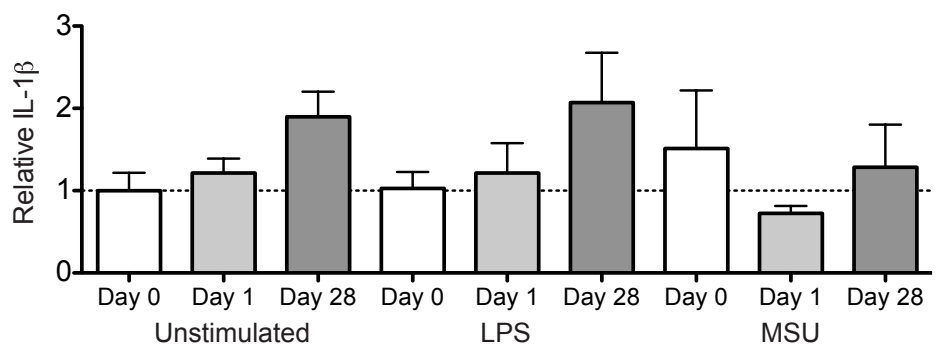

Figure 5.24 ULT increases monocyte NLRP3 inflammasome components.

Cytosolic protein was isolated from blood monocytes purified from patients before (0) and after 1 and 28 days of ULT. Monocytes were unstimulated $(n=8)$ or stimulated for $1 \mathrm{hr}$ with A: LPS $(100 \mathrm{ng} / \mathrm{ml} n=4)$ or B: $M S U(0.5 \mathrm{mg} / \mathrm{ml} n=4)$. A: NLRP3, B: proCaspase1, C: Caspase1, D: Pro-IL-1 $\beta$ and E: IL-1 $\beta$ western blot band intensity relative to $\beta$-actin and Day 0 were analysed using ImageJ. ${ }^{*} p<0.05$, t-test. Data are mean with SEM. 


\subsection{Summary}

In this chapter I have presented results from a clinical study investigating how ULT, in patients with hyperuricaemia, alters monocyte inflammatory function. Allopurinol treatment significantly lowered serum UA levels over the course of the study. Serum UA levels positively correlated with the percentage of monocytes in the blood and with monocyte CD11b expression, confirming the Harper group's previous findings comparing monocytes from normouricaemic to hyperuricaemic individuals ${ }^{128}$. I also extended this analysis, demonstrating that ULT reduced monocyte percentages and CD11b expression, thereby reinforcing the role of serum UA in the control of monocyte recruitment into the circulation and tissues.

Monocytes produced less IL-10 in response to LPS after ULT and there was a negative correlation between TNF $\alpha$ and IL-6 production and serum UA levels after MSU stimulation. Monocytes also produced more chemokines, such as CCL2, CCL5 and CCL7, following ULT and immune stimulation. These data indicated that blood monocytes were becoming more inflammatory as serum UA levels decreased.

The increased monocyte inflammatory profile was supported by western blot analysis, which showed that ULT caused an increase in MAP kinase phosphorylation and NFKB p65 and p50 nuclear translocation. ULT also increased monocyte ASC, NLRP3 and pro-caspase1 expression, the three central components of the NLRP3 inflammasome.

The study was designed primarily to measure the kinetics of allopurinol metabolism in hyperuricaemic patients with gout over 28 days, using a constant allopurinol dose of 50 or $100 \mathrm{mg} /$ day depending on patient creatinine clearance. The usual practice of titrating the dose upwards ${ }^{83}$ to achieve target serum UA levels was not instigated until after the end of the study. Therefore, the main limitation of this study was the low starting dose of allopurinol and as such, the effect of reaching the target UA level of $<0.36 \mathrm{mM}$ could not be evaluated. Other limitations include the different comorbidities of each patient, which could have unpredictable effects on monocyte function, and medications the patients were taking. Anti-inflammatory medications could influence the behaviour of immune cells and alter the systemic immune 
environment but are a normal part of regular treatment and care of gouty arthritis. Additionally, there were some strong trends in the data that may prove to be biologically significant if measured in a larger patient group.

The results of this chapter confirmed the suppressive effects of soluble UA by demonstrating that lowering of serum UA, in a clinical setting, increases the inflammatory potential of human blood monocytes. This study indicates that the suppression of monocyte inflammatory potential induced by hyperuricaemia can be reversed with pharmacological reduction of serum UA levels. 

Chapter 6:

Discussion and Future Directions 



\section{Discussion and future directions}

\subsection{Overview}

Hyperuricaemia is directly associated with metabolic syndrome, an umbrella term encompassing diseases that result from perturbations in energy metabolism. These conditions include obesity, type 2 diabetes, hypertension, ischemic heart disease and gout and are commonly associated with lowgrade chronic inflammation ${ }^{2}$.

The widely reported associations between hyperuricaemia and chronic inflammation have meant that hyperuricaemia is commonly seen as a potential inflammatory driver. However, results from a previous observational study conducted in the Harper group (figure 3.1, unpublished data) provided evidence contrary to this hypothesis. Comparing the ex vivo responses of innate immune cells from healthy controls versus people with hyperuricaemia, the study found a negative correlation between LPS-induced monocyte proinflammatory cytokine production and serum UA levels. This indicated that hyperuricaemia could in fact be moderating monocyte responses during chronic inflammation.

The aim of my research was to expand on our understanding of the potential moderating effects of soluble UA on the pro-inflammatory response of blood monocytes to stimulation with LPS. Using a combination of in vitro, in vivo and clinical modulation of soluble UA my research has confirmed the ability of soluble UA to moderate blood monocyte pro-inflammatory cytokines alongside increased production of anti-inflammatory IL-10. This occurs in conjunction with decreased pro-inflammatory cytokine signalling and upregulation of antiinflammatory signalling pathways. 


\subsection{Monocyte inflammatory signalling and cytokine production}

\subsubsection{NFKB signalling}

In monocytes the NFKB p65/p50 heterodimer is activated by LPS stimulation to trigger pro-inflammatory cytokine transcription. p65/p50 is sequestered in the cytosol by $I_{\kappa} \mathrm{B} \alpha$ and LPS stimulation triggers proteosomal degradation of $\mathrm{I}_{\kappa} \mathrm{B} \alpha$ releasing $\mathrm{p} 65 / \mathrm{p} 50$ for nuclear translocation. $\mathrm{I}_{\kappa \mathrm{B}} \alpha$ is then gradually replenished via NFKB signalling in a negative feedback loop to shut down inflammation ${ }^{202}$.

My results showed that in vitro and in vivo hyperuricaemia decreased p65 nuclear translocation in LPS-stimulated monocytes and that there was a more rapid recovery of $I_{\kappa} \mathrm{B} \alpha$ levels in the presence of hyperuricaemia. These results indicate that soluble UA could be enhancing $I_{\kappa} \mathrm{B} \alpha$ through a posttranscriptional mechanism to limit pro-inflammatory NF $\kappa B$ p65/p50 signalling and reduce pro-inflammatory cytokine production (figure 6.1).

The NFkB p50/p50 homodimer is a transcription factor for the antiinflammatory cytokine IL-10 and is also inhibitory of p65/p50 signalling ${ }^{171}$.

My data showed an increase in LPS-induced monocyte nuclear p50 in the presence of in vitro hyperuricaemia. This indicates that hyperuricaemia may be augmenting IL-10 and inhibiting pro-inflammatory p65/p50 signalling via increases in nuclear p50/p50.

The possible mechanism behind increased nuclear translocation of the p50 subunit is unclear. $\mathrm{Bcl} 3$ is a member of the ${ }_{1 \kappa} \mathrm{B}$ family of proteins that bind and inhibit NFKB transcription factors and has been implicated as an inhibitor of p50/p50-induced transcription of IL-10 201 . In the clinical study ULT coincided with an increase in nuclear $\mathrm{Bcl} 3$ expression, indicating that $\mathrm{Bcl} 3$ may be involved in the mechanism through which UA enhances LPS-induced IL-10 production. 


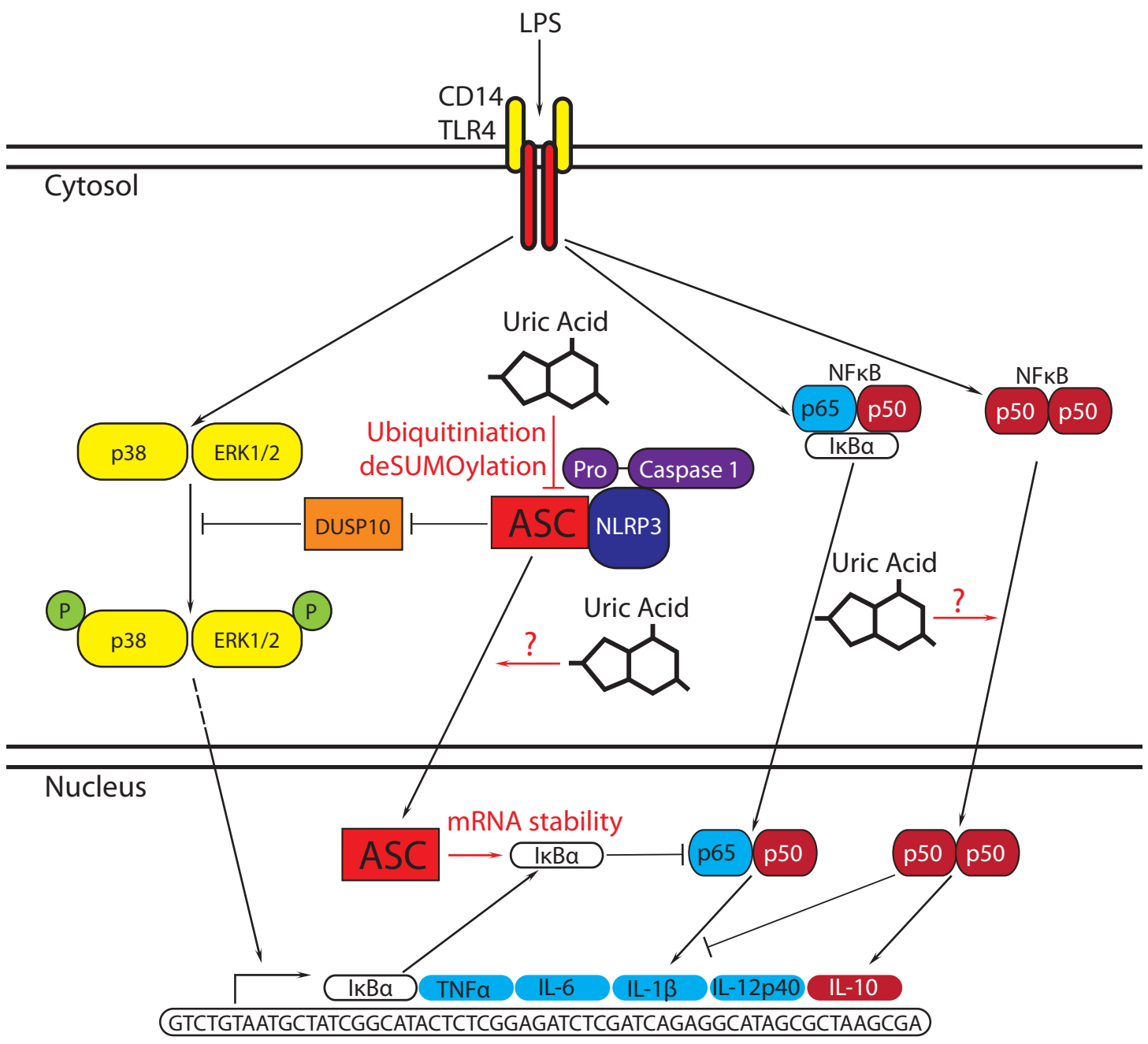

Figure 6.1 Potential mechanisms through which soluble UA suppresses monocyte inflammatory signalling.

Soluble UA could decrease ASC expression via enhanced ubiquitination or deSUMOylation, thereby removing suppression of DUSP10. Increased nuclear ASC could enhance mRNA stability to support I $\kappa \alpha$ translation. It is unclear how UA might enhance nuclear trafficking of ASC and NFKB p50. 


\subsubsection{MAP kinase signalling}

LPS activates MAP kinase phosphorylation, a key event in the signal transduction pathway that leads to pro-inflammatory cytokine production.

In my study, elevated UA levels (in vitro and in vivo) resulted in decreased p38 and ERK1/2 phosphorylation. I saw the reverse pattern in vivo with C57 mice and in the ULT study. These findings demonstrate that hyperuricaemia reduces pro-inflammatory MAP kinase signalling in activated monocytes.

A role for cytosolic ASC in MAP kinase regulation has been shown using knockdown of ASC with shRNA constructs where knockdown of ASC increased DUSP10 activity and dephosphorylation of ERK1/2 ${ }^{175}$. My data indicated that in vitro and in vivo hyperuricaemia mediate suppression of MAP kinase phosphorylation in association with increased DUSP10 and decreased ASC expression. ULT in vivo and in the clinical study had the opposite effect, increasing ASC expression alongside MAP kinase phosphorylation. These results identify soluble UA as an endogenous regulator of monocyte ASC expression and suggest that the concentration of soluble UA can control the degree of LPS-induced MAP kinase phosphorylation via modulation of ASC expression (figure 6.1).

Interestingly, ASC expression was reduced in monocytes after just $2 \mathrm{hr}$ of inducing in vitro or in vivo hyperuricaemia. This rapid reduction in cytosolic ASC suggests that soluble UA was reducing ASC expression through a posttranslational mechanism. One potential mechanism is proteosomal degradation, which involves the attachment of multiple ubiquitin molecules to cellular proteins to target them for removal by the proteosome ${ }^{203}$. There is evidence that ASC can be ubiquitinated following activation of autophagy ${ }^{204}$, providing a precedent for proteosomal degradation of ASC that could potentially be enhanced by increases in soluble UA (figure 6.1).

Alongside ubiquitination there is SUMOylation, which involves covalent attachment of small ubiquitin-like modifiers (SUMO) to target proteins. 
SUMOylation of proteins can result in many different functional outcomes including cytosol to nuclear trafficking and increased protein stability ${ }^{205,206}$. There is evidence that soluble UA can influence protein SUMOylation. One study shows that in mononuclear phagocytes overexpression of xanthine oxidoreductase (XOR) or treatment with soluble UA decreased SUMOylation of PPAR $\gamma^{207}$. However, the authors infer that increased activity of XOR rather than increased soluble UA reduces PPAR $\gamma$ SUMOylation despite showing that increased XOR and soluble UA treatment produce exactly the same result. It is therefore possible that this study demonstrates a precedent for increased soluble UA reducing protein SUMOylation. Therefore, hyperuricaemia could be causing ASC deSUMOylation and destabilisation, which may be driving the reduction in ASC observed in my research (figure 6.1).

There is evidence that ASC has an important role in the nucleus supporting mRNA stabilisation of DOCK2 in both myeloid cells and lymphocytes. This was shown by a decrease in DOCK2 mRNA transcript levels, mRNA half-life and protein expression in ASC deficient cells ${ }^{176}$. My clinical study showed that ULT resulted in increased cytosolic and decreased nuclear ASC in human monocytes indicating soluble UA may increase nuclear trafficking of ASC. Increasing nuclear translocation of ASC could support mRNA stability of $I_{\kappa} \mathrm{B} \alpha$ transcripts (figure 6.1), which would in turn reduce pro-inflammatory NFKB p65/p50 signalling after LPS stimulation. Nuclear translocation of ASC could also be a mechanism through which DUSP10 is released from inhibition by ASC in the cytosol. Whether ASC nuclear trafficking is regulated by SUMOylation is unknown.

\subsubsection{NLRP3 inflammasome signalling}

The NLRP3 inflammasome is a multiprotein complex comprised of NLRP3, ASC and pro-caspase 1 that cleaves pro-IL-1 $\beta$ into the active form. ASC is a major component of the NLRP3 inflammasome and in vitro hyperuricaemia reduced both ASC expression and LPS-induced IL-1 $\beta$. 
At the same time, induction of acute hyperuricaemia in vivo increased procaspase 1 and pro-IL-1 $\beta$ expression in splenic monocytes and this pattern was reversed in C57 mice treated with allopurinol or rasburicase. However, ULT in the clinical study increased NLRP3, pro-caspase 1 and pro-IL-1 $\beta$ expression in human blood monocytes. The reason for this differential expression pattern is unclear and may simply be a result of inherent differences in mouse and human immunology. However, in vivo ULT with rasburicase in the PLT2 strain also resulted in increased pro-caspase 1 and pro-IL-1 $\beta$ expression. In this instance ULT produced the same functional changes during chronic hyperuricaemia in mice as it had in humans. Therefore, there is some commonality in NLRP3 inflammasome expression in response to ULT and the common factor may have been the presence of chronic hyperuricaemia.

In the clinical study ULT increased NLRP3 inflammasome component expression, suggesting that the NLRP3 inflammasome would be more reactive in monocytes from a lower serum UA environment. MSU-induced pro-IL-1 $\beta$ protein expression was increased after ULT but MSU-induced IL-1 $\beta$ production was decreased. This indicates that inflammasome component expression (as measured by western blotting) does not always lead to increased cleavage and active IL-1 $\beta$ secretion (as measured by IL-1 $\beta$ ELISA).

\subsection{Monocyte adhesion molecule expression and CCL2}

CD11b is an adhesion molecule involved in the tethering of monocytes to the vascular endothelium at sites of inflammation ${ }^{208}$; ICAM1 is a ligand for CD11b and is important for monocyte-monocyte interactions and cell-cell adhesion between monocytes and other immune cells ${ }^{209}$; CCL2 is a major monocyte chemokine that can recruit monocytes from the bone marrow via the CCL2CCR2 chemokine-receptor axis ${ }^{118}$.

We have already reported that hyperuricaemic individuals have more CD14+ monocytes in the circulation versus normouricaemic controls, that the monocytes from hyperuricaemics have increased CD11b expression and that these changes are associated with increased serum CCL2 ${ }^{128}$. Another study 
involving hyperuricaemic children also reports increased CCL2 levels in hyperuricaemics versus healthy controls ${ }^{210}$. Additionally, soluble UA has been shown to induce production of CCL2 in vascular smooth muscle cells.

In my clinical study ULT had no effect on serum CCL2 levels but confirmed the positive associations between serum UA levels, blood monocyte percentages and CD11b expression. Soluble UA itself can act as a chemoattractant for eosinophils ${ }^{211}$. Therefore, it may be acting directly to recruit monocyte precursors from the bone marrow. However, this does not rule out a role for soluble UA in mediating CCL2 production. The degree of UA lowering may not have been sufficient to cause a decrease in serum CCL2, as patient serum UA levels did not reach the therapeutic target $(<0.36 \mathrm{mM})$.

The human ULT study also showed that CD11b and ICAM1 expression on LPS-activated blood monocytes positively correlated with serum UA levels. In contrast, in vitro hyperuricaemia had no effect on monocyte adhesion molecule expression regardless of LPS stimulation, although ICAM1 was not measured in vitro. This could be an example of differences between in vitro and clinical hyperuricaemia where the in vitro UA incubation times may have been too short to result in any changes in adhesion molecule expression. For example, during ULT monocytes were exposed to lower serum UA levels for up to 28 days whereas with in vitro hyperuricaemia monocytes were only treated with soluble UA for a maximum of $18 \mathrm{hr}$.

Nevertheless, my research has identified a potential mechanism whereby increased serum UA levels contribute to monocyte recruitment into the circulation and movement between the circulation and the tissues through increased expression of the adhesion molecule CD11b. To complement this increased ICAM1 expression on tubular epithelial cells in the presence of hyperuricaemia has been shown to contribute to monocyte/macrophage renal infiltration ${ }^{147}$. Additionally, increased monocyte ICAM1 expression induced by hyperuricaemia may act to increase cell-cell binding between LPS-stimulated monocytes as observed in the homotypic aggregation studies in Chapter 3. 


\subsection{Murine hyperuricaemia and urate-lowering therapy}

\subsubsection{Acute hyperuricaemia and ULT with allopurinol and rasburicase}

In vivo hyperuricaemia suppressed the systemic innate immune response to LPS and lowering serum UA in vivo produced the opposite effect. Allopurinol and rasburicase are both used clinically for the treatment of hyperuricaemia and both were equally effective at acutely lowering serum UA levels in C57 mice.

Clinically rasburicase reduces serum UA much faster than allopurinol ${ }^{54}$. Therefore, we could hypothesise that rasburicase would produce a more pronounced increase in pro-inflammatory cytokines in my model of systemic inflammation. However, rasburicase was less effective at increasing LPSinduced systemic inflammation, as measured by serum cytokine production, than allopurinol.

Additionally, the signalling response of splenic monocytes to LPS stimulation differed in allopurinol and rasburicase treated mice. Allopurinol predominantly

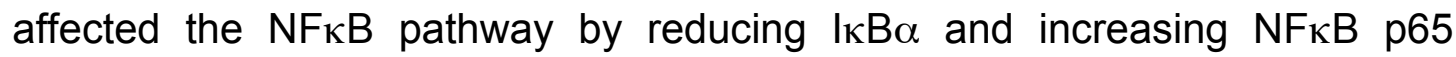
subunit nuclear translocation, while rasburicase treatment raised ASC and increased NFKB.

These differences in cytokine production and signalling may be a result of differences in the mechanism of action of allopurinol and rasburicase.

Allopurinol is a small molecule inhibitor that reduces serum UA by inhibiting intracellular UA production by $\mathrm{XOR}^{58}$. Rasburicase, a recombinant enzyme, is much larger and less likely to enter cells without being actively taken up and is therefore more likely to function as an extracellular enzyme within the circulation. Even though both treatments resulted in equivalent serum UA lowering, allopurinol may be more effective at altering immune cell function by directly reducing intracellular UA concentrations.

Additionally, rasburicase and allopurinol could have differential effects on ROS dependent inflammatory signalling ${ }^{212}$ as oxidation of UA by uricase ${ }^{213}$ 
and inhibition of XOR with allopurinol ${ }^{214}$ can increase and decrease ROS production respectively.

\subsubsection{The PLT2 mouse strain as a model for chronic hyperuricaemia}

Human hyperuricaemia is often a chronic condition and it is important to model a disease as closely as possible to avoid introducing experimental artefacts. To this end I investigated the suitability of the PLT2 strain as a model of chronic hyperuricaemia and ULT in the context of LPS-induced inflammation.

When comparing PLT2 mice to C57 mice there was a 2-fold increase in serum UA and LPS challenge increased pro-inflammatory serum cytokines and IL-10. This increase in pro-inflammatory serum cytokines in the PLT2 strain was not consistent with the effect of acute hyperuricaemia in C57 mice and did not correspond with human monocyte responses to hyperuricaemia and LPS. There were no changes in splenic monocyte inflammatory signalling pathways between PLT2 and C57 mice. The lack of changes in splenic monocyte signalling between the two mouse strains may be a result of lifelong exposure in the PLT2 strain to higher serum UA levels, causing the PLT2 mouse immune system to adjust to the chronic UA exposure.

Even though the PLT2 strain was hyperuricaemic in comparison to C57 mice, results from the PLT2 strain were not comparable to acute hyperuricaemia in C57 mice. Therefore, further research will be required to ascertain whether the PLT2 strain is a useful tool to model inflammation in the context of chronic hyperuricaemia. An important consideration will be the potentially confounding effects of other disturbances in immunological homeostasis caused by knockout of HIU hydrolase, as the PLT2 strain also has increased platelets compared to C57 mice and is predisposed to the development of liver cancer $^{90}$. 
The mechanism through which knockout of HIU hydrolase increases serum UA in the PLT2 strain is unknown but could involve product inhibition of uricase by HIU combined with naturally low renal UA excretion. I found that a single dose of allopurinol within a $3 \mathrm{hr}$ timeframe had no effect on serum UA levels or cytokine and signalling responses to LPS in the PLT2 strain. Although UA production would have been blocked by allopurinol treatment the reduced capacity for UA metabolism in the PLT2 strain could potentially explain why there was no effect of allopurinol at this time point.

Subsequent research in the Harper group has found that 7 days of allopurinol treatment, administered via the drinking water, can significantly reduce serum UA in the PLT2 strain (unpublished data). This indicates that allopurinol can lower serum UA in the PLT2 strain but that more time is required to excrete or metabolise the excess UA.

On the other hand, acute rasburicase treatment did reduce serum UA in PLT2 mice as, unlike allopurinol, addition of exogenous uricase increases UA metabolism. Rasburicase also increased inflammatory monocyte signalling. However, the degree of UA lowering in my experiments and the short time frame may not have been sufficient to fully reverse the suppressive effects of chronic hyperuricaemia and increase pro-inflammatory serum cytokines in response to LPS. 


\subsection{Modulating soluble UA as a method of controlling inflammation and disease}

In vitro, in vivo and clinical hyperuricaemia suppressed monocyte proinflammatory cytokine production in response to LPS and, importantly, increased IL-10 production. I also showed clinically that monocyte number and adhesion molecule expression increase with increasing hyperuricaemia. Together these results demonstrate a suppressive effect of soluble UA on systemic and monocyte cytokine production and an augmentation of monocyte migratory ability. The potential consequences of these findings in the context of hyperuricaemia and different disease processes are discussed here.

\subsubsection{Hyperuricaemia and sepsis}

I have shown that in vivo pharmacomanipulation of serum UA has the potential to influence inflammation during low dose LPS but whether hyperuricaemia is protective or exacerbates sepsis is still not clear. In human studies there are reports of gross immunosuppression in tissues from septic patients analysed post-mortem ${ }^{215,216}$ and serum UA has been shown to increase in patients with sepsis, which correlates with the severity of disease $^{217}$. In mouse models increased proinflammatory cytokines and decreased IL-10 levels are associated with increased mortality during peritonitis $^{218}$ and in a model of neutropenia that causes hyperuricaemia lowering serum UA with allopurinol was shown to increase survival in LPS challenged mice ${ }^{219}$.

Taken together, the current literature provides evidence supporting a role for hyperuricaemia in exacerbating sepsis by contributing to immunosuppression. Consequently, ULT to reduce or prevent immunosuppression during sepsis could potentially aid in the inflammatory cytokine response and clearance or killing of bacteria. 


\subsubsection{Hyperuricaemia and metabolic syndrome}

The physiological functions of hyperuricaemia have been widely investigated but there is still debate over the contribution of hyperuricaemia to metabolic dysfunction ${ }^{2}$. My findings may shed some light on how hyperuricaemia moderates or contributes to these diseases from an immunological perspective.

The formation and exacerbation of cardiovascular disease is a process involving monocyte recruitment to damaged vascular endothelium, differentiation into macrophages and phagocytosis of cholesterol crystals ${ }^{127}$. Whether this process results in enlargement or regression of the atherosclerotic plaque is dependent on a multitude of factors including the phenotype of the resident macrophage ${ }^{220}$

Obesity is characterised by chronic low-grade inflammation, endocrine imbalance and increased adipose tissue macrophages. Hyperuricaemia has been shown to contribute to monocyte recruitment to adipose tissue and insulin resistance $e^{72,221}$.

Results from the ULT study indicate that there are increased monocyte numbers in the circulation during hyperuricaemia with a greater potential for adhesion and migration through increased expression of adhesion molecules. This indicates that hyperuricaemia may directly contribute to the development and severity of cardiovascular disease and obesity through increased monocyte recruitment.

However, the development of hyperuricaemia during cardiovascular disease or obesity could be a regulatory mechanism designed to counteract the chronic inflammation. Hyperuricaemia could enhance recruitment of suppressive monocytes into atherosclerotic plaques or adipose tissue in an attempt to resolve the chronic inflammation. 


\subsubsection{Soluble versus crystalline uric acid}

The results of this thesis demonstrate that soluble UA has a suppressive effect on monocyte inflammatory potential. On the other side of the spectrum is the well-established pro-inflammatory nature of crystalline UA (MSU). MSU is a powerful immune adjuvant that triggers pro-inflammatory cytokine production, NLRP3 inflammasome activation and dendritic cell maturation to promote both innate and adaptive immune responses ${ }^{77,114,222,223}$.

By integrating these two opposing functions of soluble and crystalline UA I have developed a theory that positions UA as a key regulator of local inflammation that can direct the switch from innate to adaptive immunity (figure 6.2).

Local tissue environments can become hyperuricaemic as a result of cell death induced by injury or infection, as soluble UA is rapidly released from dying cells ${ }^{107}$. My in vitro, in vivo and clinical study results all indicate that monocytes entering a tissue environment with high levels of soluble UA would be less inflammatory upon stimulation with damage associated or pathogen associated molecular patterns (DAMPs or PAMPs) present due to the injury or infection.

This local rise in soluble UA could be important for containing the immune response during low-level damage or a minor infection (figure 6.2). Increased soluble UA levels may also aid in the migration of monocytes towards the site of inflammation by increasing adhesion molecule expression on both monocytes and cells in the affected tissue ${ }^{147}$.

However, if the damage or infection were severe enough the tissue would become supersaturated with soluble UA to the point where UA crystallisation occurs in order to activate a potent inflammatory response (figure 6.2).

In this way soluble UA could act as an inhibitory danger signal that discourages an overactive innate immune response or unnecessary adaptive immune response to a minor insult and limits the chances of a break in peripheral tolerance. The crystal switch to MSU would occur when the innate 
response is unable to contain the damage and would facilitate the development of potent innate and adaptive immune responses (figure 6.2).

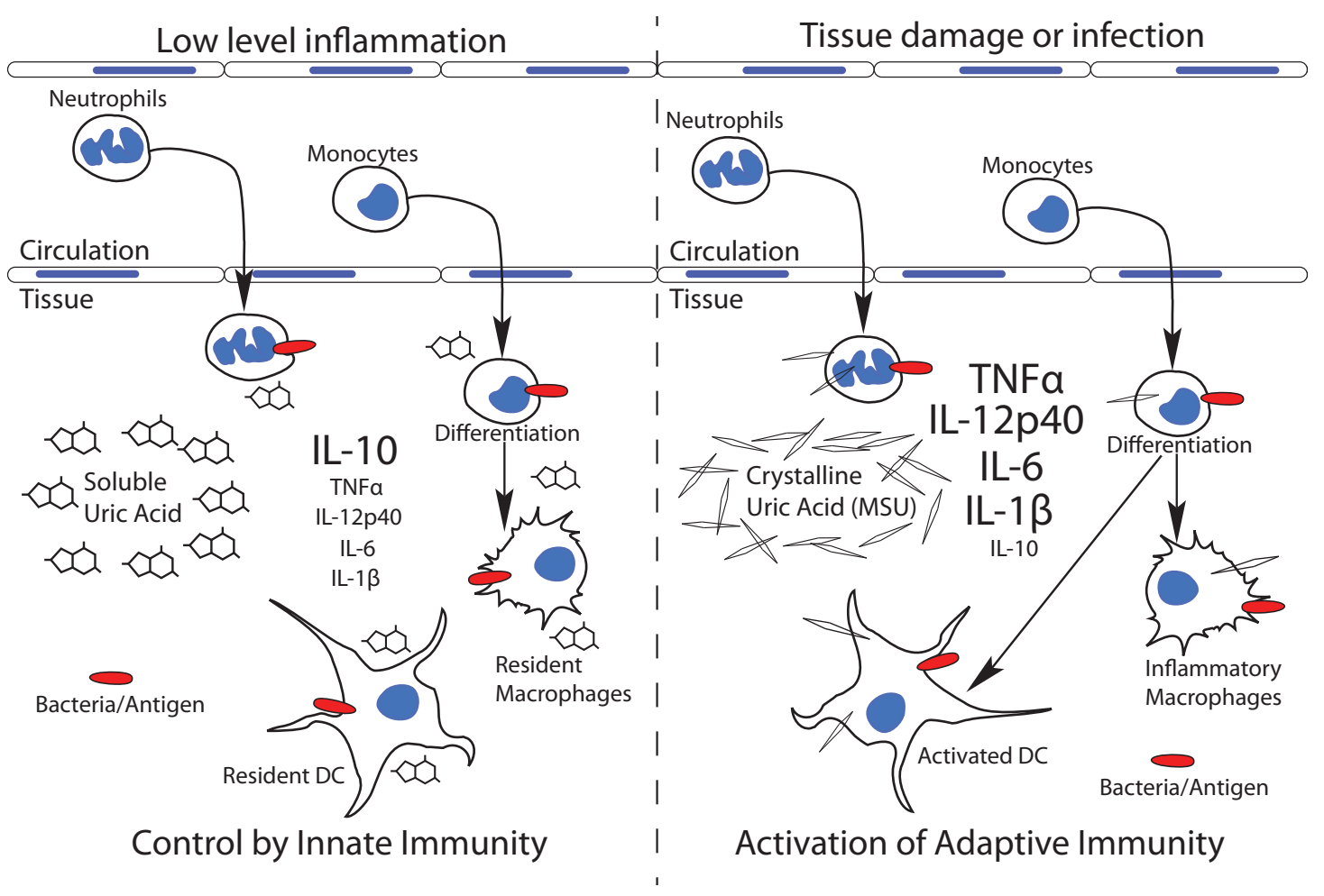

Figure 6.2 Proposed model of soluble UA controlling low-level inflammation and the switch to crystalline UA (MSU) activating innate and adaptive immunity during severe inflammation.

The font size of cytokines reflects their relative concentration. 


\subsection{Future directions}

\subsubsection{Immune cell signalling}

I have shown that soluble UA modulates monocyte signalling during inflammation. Therefore, the next question is how does UA specifically interact with these signalling molecules. It is clear that soluble UA affects several inflammatory signalling pathways; namely the MAP kinase, NFKB and the NLRP3 inflammasome; but whether UA has a single mechanism of action that is broad reaching or alters the different pathways through individual interactions is not known.

There are indications that soluble UA may be involved in protein ubiquitination or deSUMOylation, although there is no direct evidence that soluble UA can enhance ASC ubiquitination and there are no reports yet of ASC SUMOylation. These modifications are functionally diverse and could potentially explain the broad impact of soluble UA across the different inflammatory signalling pathways. Co-immunoprecipitation experiments, using protein isolated from monocytes treated with soluble UA, could be used to extract ASC. Western blotting could then be used to determine whether soluble UA alters ubiquitination or SUMOylation of ASC. Other potential target proteins such as NLRP3 and DUSP10 could also be investigated for ubiquitination and SUMOylation levels in the presence of hyperuricaemia.

The majority of this work involved stimulation of the monocyte TLR4 pathway with LPS. This has dictated, to some extent, the signalling pathways I have investigated. However, these signalling pathways are not unique to monocytes or LPS stimulation. Additionally, inflammasome independent functions of ASC are not unique to monocytes, having been demonstrated in dendritic cells and T-cells ${ }^{177,224}$. The effect of soluble UA on cytokine production and antigen presentation in myeloid cells, and the proliferation and antibody production of $\mathrm{T}$ and B-cells would be an excellent starting point to 
broaden this line of research and determine the extent of soluble UA's role in immune cell modulation.

\subsubsection{Adhesion and migration}

The clinical study confirmed that hyperuricaemia is augmenting monocyte recruitment into the circulation and also increasing adhesion molecule expression on these monocytes.

I did not measure monocyte ICAM1 expression in vitro but the clinical study suggests that the increased monocyte homotypic aggregation I observed with in vitro hyperuricaemia and LPS stimulation could be a result of increased ICAM1 expression through its interaction with $C D 11 b^{192}$. This could be investigated by measuring ICAM1 expression on monocytes following in vitro hyperuricaemia and LPS stimulation in combination with blocking antibodies for ICAM1 and CD11b. This could be followed up with antibody labelling of CD11b and ICAM1 and fluorescent microscopy to determine whether these molecules colocalise at the point of monocyte-monocyte interaction.

The next experimental step could be in vivo analysis of monocyte migration into tissues and the contribution of adhesion molecules to this process. The migratory potential could be investigated by measuring monocyte infiltration in mouse models of local inflammation, such as peritonitis or atherosclerosis, combined with hyperuricaemia or ULT.

Measuring rolling and tethering of monocytes under laminar flow and in the presence of hyperuricaemia could be used to determine whether the increased adhesion molecule expression translates to a quantifiable gain of adhesive function. These experiments could be combined with blocking different adhesion molecules to determine the extent of the contribution of each.

In this way monocyte recruitment into the tissues could be measured alongside their ability to adhere to and cross the vascular endothelium in response to different inflammatory stimuli and changes to circulating soluble UA levels. 


\subsubsection{Murine hyperuricaemia and urate-lowering therapy}

Pharmacologically induced acute hyperuricaemia was sufficient to induce a suppressive inflammatory response to systemic LPS challenge. However, C57 mice with acute hyperuricaemia responded differently than PLT2 mice with chronic hyperuricaemia to LPS challenge. This may be a reflection of different effects of acute vs. chronic hyperuricaemia. To study this further pharmacological induction of chronic hyperuricaemia in C57 mice could be compared to genetic hyperuricaemia in the PLT2 strain to ascertain the suitability of the PLT2 strain as a model of chronic hyperuricaemia. As human hyperuricaemia is predominantly chronic it would be important to validate a chronic model of hyperuricaemia for use in further studies.

Well-defined and validated chronic models of hyperuricaemia would then allow for the development of chronic ULT models combined with immune challenge. C57 mice with acute ULT responded to LPS with a reversal of the inflammatory phenotype produced by acute hyperuricaemia and LPS. However, monocyte immune suppression caused by chronic hyperuricaemia may be more profound than those caused by acute hyperuricaemia and could require long-term ULT to achieve a phenotypic reversal.

The PLT2 strain was refractory to acute allopurinol treatment but preliminary results showed that 1 week of allopurinol treatment via the drinking water lowered PLT2 mouse serum UA. The next step would be to investigate the effect of chronic ULT on inflammation in the PLT2 strain.

The differential effects of rasburicase and allopurinol on monocyte signalling molecules provides a rationale for combination therapy as there may be an additive or even synergistic effect on immune cell responses to inflammation when both therapies are used together. For example, the rapid urate lowering effects of an initial bolus rasburicase dose could be followed up with long-term allopurinol treatment to maintain urate lowering and ensure intracellular UA is lowered efficiently. If reducing serum UA also increases the inflammatory potential of other immune cells then different ULT dosing schedules could be investigated to optimise the augmentation of inflammatory responses in different cell types. 


\subsubsection{Human urate-lowering therapy studies}

The human ULT study was not initially designed to determine the effects of serum UA levels on immune cell function. This meant that lowering UA was not the main goal of the study and the final measurement (day 28) was taken before the patients reached target serum UA levels $(<0.36 \mathrm{mM})$. It would be ideal to resample the 15 patients from the ULT study once they have reached $0.36 \mathrm{mM}$ to compare the suppressive effect of hyperuricaemia to normouricaemia. This could be combined with the sampling and analysis of 15 healthy, age and gender matched controls to see whether the suppressive effects of hyperuricaemia on monocyte function are truly plastic. In this case we could hypothesise that monocyte immune function in patients who have reached target UA levels would return to normal and be equivalent to that of monocytes from healthy controls.

Currently ULT is only prescribed for hyperuricaemia associated with gout or tumour lysis syndrome. My ULT study showed that lowering serum UA levels increased the inflammatory potential of blood monocytes but decreased blood monocyte numbers and migratory potential. These results provide a rationale for the treatment of hyperuricaemia associated with diseases where monocyte infiltration is a known mediator of the pathophysiology, such as cardiovascular disease and obesity ${ }^{72,220}$. This could begin with an allopurinol safety and efficacy trial enrolling normo- and hyperuricaemic participants who are otherwise healthy or suffer from different metabolic syndrome related diseases. It could be expanded to include other methods of ULT and it could also look at other circulating immune cells in the context of their inflammatory potential to cell specific stimuli. It would be important to include measurements of patient disease burden and recovery to establish whether these parameters are linked to changes in serum UA and immune cell function. Finally, extreme care should be taken to carefully define and evaluate safety parameters and primary outcomes as my results also indicate that ULT could potentially exacerbate inflammation by removing the suppressive effects of hyperuricaemia. 


\section{References}

1. Storey, G. D. Alfred Baring Garrod (1819-1907). Rheumatology (Oxford) 40, 1189-1190 (2001).

2. Alvarez-Lario, B. \& Macarron-Vicente, J. Is there anything good in uric acid? QJM 104, 1015-1024 (2011).

3. Gustafsson, D. \& Unwin, R. The pathophysiology of hyperuricaemia and its possible relationship to cardiovascular disease, morbidity and mortality. BMC Nephrol 14, 164 (2013).

4. Ramazzina, I., Folli, C., Secchi, A., Berni, R. \& Percudani, R. Completing the uric acid degradation pathway through phylogenetic comparison of whole genomes. Nat Chem Biol 2, 144-148 (2006).

5. Wu, X. W., Muzny, D. M., Lee, C. C. \& Caskey, C. T. Two independent mutational events in the loss of urate oxidase during hominoid evolution. J. Mol. Evol. 34, 78-84 (1992).

6. Fathallah-Shaykh, S. A. \& Cramer, M. T. Uric acid and the kidney. Pediatr. Nephrol. (2013). doi:10.1007/s00467-013-2549-x

7. Alvarez-Lario, B. \& Macarron-Vicente, J. Uric acid and evolution. Rheumatology (Oxford) 49, 2010-2015 (2010).

8. Zoccali, C. \& Mallamaci, F. Uric Acid, Hypertension, and Cardiovascular and Renal Complications. Curr. Hypertens. Rep. (2013). doi:10.1007/s11906-013-0391-y

9. Ames, B. N., Cathcart, R., Schwiers, E. \& Hochstein, P. Uric acid provides an antioxidant defense in humans against oxidant- and radical-caused aging and cancer: a hypothesis. Proc Natl Acad Sci USA 78, 6858-6862 (1981).

10. Shen, C., Guo, Y., Luo, W., Lin, C. \& Ding, M. Serum urate and the risk of Parkinson's disease: results from a meta-analysis. Can $J$ Neurol Sci 40, 73-79 (2013).

11. Schrag, M. et al. Oxidative stress in blood in Alzheimer's disease and mild cognitive impairment: A meta-analysis. Neurobiol. Dis. 59, 100110 (2013).

12. Spitsin, S. V. et al. Comparison of uric acid and ascorbic acid in protection against EAE. Free Radic Biol Med 33, 1363-1371 (2002).

13. Zamani, A., Rezaei, A., Khaeir, F. \& Hooper, D. C. Serum and cerebrospinal fluid uric acid levels in multiple sclerosis patients. Clin Neurol Neurosurg 110, 642-643 (2008).

14. Liu, B. et al. Serum uric acid levels in patients with multiple sclerosis: a meta-analysis. Neurol. Res. 34, 163-171 (2012).

15. Ashtari, F., Bahar, M., Aghaei, M. \& Zahed, A. Serum uric acid level in patients with relapsing-remitting multiple sclerosis. J Clin Neurosci 20, 676-678 (2013).

16. Markowitz, C. et al. The treatment of multiple sclerosis with inosine. The Journal of Alternative and Complementary Medicine 15, 619-625 (2009).

17. Gonsette, R. E. et al. Boosting endogenous neuroprotection in multiple sclerosis: the ASsociation of Inosine and Interferon beta in relapsingremitting Multiple Sclerosis (ASIIMS) trial. Mult. Scler. 16, 455-462 
(2010).

18. Santos, C. X., Anjos, E. I. \& Augusto, O. Uric acid oxidation by peroxynitrite: multiple reactions, free radical formation, and amplification of lipid oxidation. Arch Biochem Biophys 372, 285-294 (1999).

19. Meotti, F. C. et al. Urate as a physiological substrate for myeloperoxidase: implications for hyperuricemia and inflammation. Journal of Biological Chemistry 286, 12901-12911 (2011).

20. Guzik, T. J., Korbut, R. \& Adamek-Guzik, T. Nitric oxide and superoxide in inflammation and immune regulation. $J$ Physiol Pharmacol 54, 469-487 (2003).

21. Chen, A. C.-H. et al. Low-level laser therapy activates NF-kB via generation of reactive oxygen species in mouse embryonic fibroblasts. PLoS ONE 6, e22453 (2011).

22. Enomoto, A. et al. Molecular identification of a renal urate anion exchanger that regulates blood urate levels. Nature 417, 447-452 (2002).

23. Tasic, V. et al. Clinical and functional characterization of URAT1 variants. PLoS ONE 6, e28641 (2011).

24. Stiburkova, B., Taylor, J., Marinaki, A. M. \& Sebesta, I. Acute kidney injury in two children caused by renal hypouricaemia type 2. Pediatr. Nephrol. 27, 1411-1415 (2012).

25. Igarashi, T., Sekine, T., Sugimura, H., Hayakawa, H. \& Arayama, T. Acute renal failure after exercise in a child with renal hypouricaemia. Pediatr. Nephrol. 7, 292-293 (1993).

26. Kurihara, I. et al. A case of exercise-induced acute renal failure in a patient with enhanced renal hypouricaemia. Nephrol Dial Transplant 15, 104-106 (2000).

27. Yim, J. J. et al. Exercise-induced acute renal failure in a patient with congenital renal hypouricaemia. Nephrol Dial Transplant 13, 994-997 (1998).

28. Ohta, T. et al. Exercise-induced acute renal failure associated with renal hypouricaemia: results of a questionnaire-based survey in Japan. Nephrol Dial Transplant 19, 1447-1453 (2004).

29. Uaratanawong, S., Suraamornkul, S., Angkeaw, S. \& Uaratanawong, R. Prevalence of hyperuricemia in Bangkok population. Clin. Rheumatol. 30, 887-893 (2011).

30. Mikuls, T. R. et al. Gout epidemiology: results from the UK General Practice Research Database, 1990-1999. Ann Rheum Dis 64, 267272 (2005).

31. Qiu, L. et al. Prevalence of hyperuricemia and its related risk factors in healthy adults from Northern and Northeastern Chinese provinces. BMC Public Health 13, 664 (2013).

32. Adamopoulos, D., Vlassopoulos, C., Seitanides, B., Contoyiannis, P. \& Vassilopoulos, $\mathrm{P}$. The relationship of sex steroids to uric acid levels in plasma and urine. Acta Endocrinol 85, 198-208 (1977).

33. Hak, A. E. \& Choi, H. K. Menopause, postmenopausal hormone use and serum uric acid levels in US women--the Third National Health and Nutrition Examination Survey. Arthritis Res Ther 10, R116 (2008).

34. Kurahashi, H. et al. Testosterone replacement elevates the serum uric 
acid levels in patients with female to male gender identity disorder. Endocr. J. (2013).

35. Saag, K. \& Choi, H. Epidemiology, risk factors, and lifestyle modifications for gout. Arthritis Res Ther 8, S2 (2006).

36. Caulfield, M. J. et al. SLC2A9 is a high-capacity urate transporter in humans. PLoS Med. 5, e197 (2008).

37. Woodward, O. M. et al. Identification of a urate transporter, ABCG2, with a common functional polymorphism causing gout. Proc Natl Acad Sci USA 106, 10338-10342 (2009).

38. Hollis-Moffatt, J. E. et al. Role of the urate transporter SLC2A9 gene in susceptibility to gout in New Zealand Māori, Pacific Island, and Caucasian case-control sample sets. Arthritis Rheum 60, 3485-3492 (2009).

39. Phipps-Green, A. J. et al. A strong role for the ABCG2 gene in susceptibility to gout in New Zealand Pacific Island and Caucasian, but not Māori, case and control sample sets. Hum. Mol. Genet. 19, 4813-4819 (2010).

40. Zhang, L. et al. Association of Functional Polymorphism rs2231142 (Q141K) in the ABCG2 Gene With Serum Uric Acid and Gout in 4 US Populations: The PAGE Study. Am. J. Epidemiol. (2013). doi:10.1093/aje/kws330

41. Ichida, K. et al. Decreased extra-renal urate excretion is a common cause of hyperuricemia. Nat Commun 3, 764 (2012).

42. Villegas, R. et al. Purine-rich foods, protein intake, and the prevalence of hyperuricemia: the Shanghai Men's Health Study. Nutr Metab Cardiovasc Dis 22, 409-416 (2012).

43. Nakamura, K. et al. Alcohol intake and the risk of hyperuricaemia: a 6year prospective study in Japanese men. Nutr Metab Cardiovasc Dis 22, 989-996 (2012).

44. Teng, G. G. et al. Serum urate levels and consumption of common beverages and alcohol among chinese in singapore. Arthritis Care Res (Hoboken) 65, 1432-1440 (2013).

45. Puig, J. G. \& Fox, I. H. Ethanol-induced activation of adenine nucleotide turnover. Evidence for a role of acetate. $J$ Clin Invest 74, 936-941 (1984).

46. Lieber, C. S., Jones, D. P., Losowsky, M. S. \& Davidson, C. S. Interrelation of uric acid and ethanol metabolism in man. $J$ Clin Invest 41, 1863-1870 (1962).

47. Hallfrisch, J., Ellwood, K., Michaelis, O. E., Reiser, S. \& Prather, E. S. Plasma fructose, uric acid, and inorganic phosphorus responses of hyperinsulinemic men fed fructose. J Am Coll Nutr 5, 61-68 (1986).

48. Choi, J. W. J., Ford, E. S., Gao, X. \& Choi, H. K. Sugar-sweetened soft drinks, diet soft drinks, and serum uric acid level: the Third National Health and Nutrition Examination Survey. Arthritis Rheum 59, 109-116 (2008).

49. Bobridge, K. S. et al. Dietary fructose in relation to blood pressure and serum uric acid in adolescent boys and girls. J Hum Hypertens 27, 217-224 (2013).

50. Hallfrisch, J. Metabolic effects of dietary fructose. FASEB J 4, 26522660 (1990). 
51. Batt, C. et al. Sugar-sweetened beverage consumption: a risk factor for prevalent gout with SLC2A9 genotype-specific effects on serum urate and risk of gout. Ann Rheum Dis (2013). doi:10.1136/annrheumdis-2013-203600

52. Dessein, P. H., Shipton, E. A., Stanwix, A. E., Joffe, B. I. \& Ramokgadi, $\mathrm{J}$. Beneficial effects of weight loss associated with moderate calorie/carbohydrate restriction, and increased proportional intake of protein and unsaturated fat on serum urate and lipoprotein levels in gout: a pilot study. Ann Rheum Dis 59, 539-543 (2000).

53. Yamashita, S., Matsuzawa, Y., Tokunaga, K., Fujioka, S. \& Tarui, S. Studies on the impaired metabolism of uric acid in obese subjects: marked reduction of renal urate excretion and its improvement by a low-calorie diet. Int J Obes 10, 255-264 (1986).

54. Cortes, J. et al. Control of plasma uric acid in adults at risk for tumor Lysis syndrome: efficacy and safety of rasburicase alone and rasburicase followed by allopurinol compared with allopurinol alone-results of a multicenter phase III study. J. Clin. Oncol. 28, 4207-4213 (2010).

55. Hochberg, J. \& Cairo, M. S. Tumor lysis syndrome: current perspective. Haematologica 93, 9-13 (2008).

56. Feig, D. I., Soletsky, B. \& Johnson, R. J. Effect of allopurinol on blood pressure of adolescents with newly diagnosed essential hypertension: a randomized trial. JAMA 300, 924-932 (2008).

57. Feig, D. I., Kang, D.-H. \& Johnson, R. J. Uric acid and cardiovascular risk. N Engl J Med 359, 1811-1821 (2008).

58. Johnson, R. J. et al. What are the key arguments against uric Acid as a true risk factor for hypertension? Hypertension 61, 948-951 (2013).

59. Hong, Q. et al. Hyperuricemia induces endothelial dysfunction via mitochondrial $\mathrm{Na}+/ \mathrm{Ca} 2+$ exchanger-mediated mitochondrial calcium overload. Cell Calcium 51, 402-410 (2012).

60. Park, J.-H. et al. Uric acid attenuates nitric oxide production by decreasing the interaction between endothelial nitric oxide synthase and calmodulin in human umbilical vein endothelial cells: A mechanism for uric acid-induced cardiovascular disease development. Nitric Oxide 32, 36-42 (2013).

61. Papežíková, I. et al. Uric acid modulates vascular endothelial function through the down regulation of nitric oxide production. Free Radic Res 47, 82-88 (2013).

62. Perticone, F. et al. Prognostic significance of endothelial dysfunction in hypertensive patients. Circulation 104, 191-196 (2001).

63. Ndrepepa, G. et al. Prognostic value of uric acid in patients with Type 2 diabetes mellitus and coronary artery disease. Clin. Sci. 124, 259-268 (2012).

64. Krishnan, E. et al. Relative and attributable diabetes risk associated with hyperuricemia in US veterans with gout. QJM 106, 721-729 (2013).

65. Johnson, R. J. et al. Hypothesis: could excessive fructose intake and uric acid cause type 2 diabetes? Endocr. Rev. 30, 96-116 (2009).

66. Perticone, F. et al. Interaction between uric acid and endothelial dysfunction predicts new onset of diabetes in hypertensive patients. 
Int. J. Cardiol. 167, 232-236 (2013).

67. Zhang, Y. et al. Uric acid induces oxidative stress and growth inhibition by activating adenosine monophosphate-activated protein kinase and extracellular signal-regulated kinase signal pathways in pancreatic $\beta$ cells. Mol. Cell. Endocrinol. 375, 89-96 (2013).

68. Taniguchi, Y. et al. Serum uric acid and the risk for hypertension and Type 2 diabetes in Japanese men: The Osaka Health Survey. J. Hypertens. 19, 1209-1215 (2001).

69. Mangge, $\mathrm{H}$. et al. Uric acid best predicts metabolically unhealthy obesity with increased cardiovascular risk in youth and adults. Obesity (Silver Spring) 21, E71-7 (2013).

70. Tsushima, Y. et al. Uric Acid secretion from adipose tissue and its increase in obesity. Journal of Biological Chemistry 288, 27138-27149 (2013).

71. Sautin, Y. Y., Nakagawa, T., Zharikov, S. \& Johnson, R. J. Adverse effects of the classic antioxidant uric acid in adipocytes: NADPH oxidase-mediated oxidative/nitrosative stress. Am J Physiol, Cell Physiol 293, C584-96 (2007).

72. Baldwin, W. et al. Hyperuricemia as a mediator of the proinflammatory endocrine imbalance in the adipose tissue in a murine model of the metabolic syndrome. Diabetes 60, 1258-1269 (2011).

73. Palmer, T. M. et al. Association of plasma uric acid with ischaemic heart disease and blood pressure: mendelian randomisation analysis of two large cohorts. BMJ 347, f4262 (2013).

74. Pfister, R. et al. No evidence for a causal link between uric acid and type 2 diabetes: a Mendelian randomisation approach. Diabetologia 54, 2561-2569 (2011).

75. Lyngdoh, T. et al. Serum uric acid and adiposity: deciphering causality using a bidirectional Mendelian randomization approach. PLOS ONE 7, e39321 (2012).

76. Dalbeth, N. \& Haskard, D. O. Mechanisms of inflammation in gout. Rheumatology (Oxford) 44, 1090-1096 (2005).

77. Martin, W. J. \& Harper, J. L. Innate inflammation and resolution in acute gout. Immunol Cell Biol 88, 15-19 (2010).

78. Campion, E. W., Glynn, R. J. \& DeLabry, L. O. Asymptomatic hyperuricemia. Risks and consequences in the Normative Aging Study. Am. J. Med. 82, 421-426 (1987).

79. Nomura, J. et al. Febuxostat, an Inhibitor of Xanthine Oxidase, Suppresses Lipopolysaccharide-Induced MCP-1 Production via MAPK Phosphatase-1-Mediated Inactivation of JNK. PLOS ONE 8, e75527 (2013).

80. George, J. \& Struthers, A. D. Role of urate, xanthine oxidase and the effects of allopurinol in vascular oxidative stress. Vascular health and risk management 5, 265-272 (2009).

81. Baker, J. F. \& Schumacher, H. R. Update on gout and hyperuricemia. Int J Clin Pract 64, 371-377 (2010).

82. Ramasamy, S. N. et al. Allopurinol Hypersensitivity: A Systematic Review of All Published Cases, 1950-2012. Drug Saf 36, 953-980 (2013).

83. Khanna, D. et al. 2012 American College of Rheumatology guidelines 
for management of gout. Part 1: systematic nonpharmacologic and pharmacologic therapeutic approaches to hyperuricemia. Arthritis Care Res (Hoboken) 64, 1431-1446 (2012).

84. Takano, Y. et al. Selectivity of febuxostat, a novel non-purine inhibitor of xanthine oxidase/xanthine dehydrogenase. Life Sci 76, 1835-1847 (2005).

85. Becker, M. A. et al. Febuxostat compared with allopurinol in patients with hyperuricemia and gout. N Engl J Med 353, 2450-2461 (2005).

86. Becker, M. A., Macdonald, P. A., Hunt, B. J. \& Jackson, R. L. Diabetes and gout: efficacy and safety of febuxostat and allopurinol. Diabetes Obes Metab 15, 1049-1055 (2013).

87. Goldfarb, D. S., MacDonald, P. A., Gunawardhana, L., Chefo, S. \& McLean, L. Randomized Controlled Trial of Febuxostat Versus Allopurinol or Placebo in Individuals with Higher Urinary Uric Acid Excretion and Calcium Stones. Clin J Am Soc Nephrol (2013). doi:10.2215/CJN.01760213

88. Kobayashi, S., Ogura, M. \& Hosoya, T. Acute neutropenia associated with initiation of febuxostat therapy for hyperuricaemia in patients with chronic kidney disease. J Clin Pharm Ther 38, 258-261 (2013).

89. Gandhi, P. K., Gentry, W. M. \& Bottorff, M. B. Cardiovascular thromboembolic events associated with febuxostat: investigation of cases from the FDA adverse event reporting system database. Seminars in arthritis and rheumatism 42, 562-566 (2013).

90. Stevenson, W. S. et al. Deficiency of 5-hydroxyisourate hydrolase causes hepatomegaly and hepatocellular carcinoma in mice. Proc Natl Acad Sci USA 107, 16625-16630 (2010).

91. Garay, R. P., El-Gewely, M. R., Labaune, J.-P. \& Richette, P. Therapeutic perspectives on uricases for gout. Joint, bone, spine : revue du rhumatisme 79, 237-242 (2012).

92. Dave, A. J., Kelly, V. M. \& Krishnan, E. Pegloticase and the patient with treatment-failure gout. Expert Rev Clin Pharmacol 5, 501-508 (2012).

93. Stamp, L. K., O'Donnell, J. L. \& Chapman, P. T. Emerging therapies in the long-term management of hyperuricaemia and gout. Intern Med $\mathrm{J}$ 37, 258-266 (2007).

94. Perez-Ruiz, F. et al. Efficacy of allopurinol and benzbromarone for the control of hyperuricaemia. A pathogenic approach to the treatment of primary chronic gout. Ann Rheum Dis 57, 545-549 (1998).

95. Hamada, T. et al. Uricosuric action of losartan via the inhibition of urate transporter 1 (URAT 1) in hypertensive patients. Am J Hypertens 21, 1157-1162 (2008).

96. Lee, M.-H. H., Graham, G. G., Williams, K. M. \& Day, R. O. A benefitrisk assessment of benzbromarone in the treatment of gout. Was its withdrawal from the market in the best interest of patients? Drug Saf 31, 643-665 (2008).

97. Pui, K., Gow, P. J. \& Dalbeth, N. Efficacy and tolerability of probenecid as urate-lowering therapy in gout; clinical experience in highprevalence population. J. Rheumatol. 40, 872-876 (2013).

98. Choi, H. K., Soriano, L. C., Zhang, Y. \& Rodríguez, L. A. G. Antihypertensive drugs and risk of incident gout among patients with 
hypertension: population based case-control study. BMJ 344, d8190 (2012).

99. Zhu, Y., Pandya, B. J. \& Choi, H. K. Comorbidities of gout and hyperuricemia in the US general population: NHANES 2007-2008. Am. J. Med. 125, 679-687.e1 (2012).

100. Juraschek, S. P., Miller, E. R. \& Gelber, A. C. Effect of oral vitamin C supplementation on serum uric acid: a meta-analysis of randomized controlled trials. Arthritis Care Res (Hoboken) 63, 1295-1306 (2011).

101. Frei, B. \& Lawson, S. Vitamin C and cancer revisited. Proc Natl Acad Sci USA 105, 11037-11038 (2008).

102. Nuki, G. \& Simkin, P. A. A concise history of gout and hyperuricemia and their treatment. Arthritis Res Ther 8 Suppl 1, S1 (2006).

103. Roubenoff, R. Gout and hyperuricemia. Rheum. Dis. Clin. North Am. 16, 539-550 (1990).

104. Klemp, P., Stansfield, S. A., Castle, B. \& Robertson, M. C. Gout is on the increase in New Zealand. Ann Rheum Dis 56, 22-26 (1997).

105. Choi, H. K. A prescription for lifestyle change in patients with hyperuricemia and gout. Curr Opin Rheumatol 22, 165-172 (2010).

106. Singh, J. A., Reddy, S. G. \& Kundukulam, J. Risk factors for gout and prevention: a systematic review of the literature. Curr Opin Rheumatol 23, 192-202 (2011).

107. Shi, Y., Evans, J. E. \& Rock, K. L. Molecular identification of a danger signal that alerts the immune system to dying cells. Nature 425, 516521 (2003).

108. Kuhn, S. et al. Increased numbers of monocyte-derived dendritic cells during successful tumor immunotherapy with immune-activating agents. The Journal of Immunology 191, 1984-1992 (2013).

109. Scott, P., Ma, H., Viriyakosol, S., Terkeltaub, R. \& Liu-Bryan, R. Engagement of CD14 mediates the inflammatory potential of monosodium urate crystals. J Immunol 177, 6370-6378 (2006).

110. Barabé, F., Gilbert, C., Liao, N., Bourgoin, S. G. \& Naccache, P. H. Crystal-induced neutrophil activation VI. Involvment of FcgammaRIIIB (CD16) and CD11b in response to inflammatory microcrystals. FASEB $J$ 12, 209-220 (1998).

111. Ng, G. et al. Receptor-independent, direct membrane binding leads to cell-surface lipid sorting and Syk kinase activation in dendritic cells. Immunity 29, 807-818 (2008).

112. Shi, Y., Mucsi, A. D. \& Ng, G. Monosodium urate crystals in inflammation and immunity. Immunol Rev 233, 203-217 (2010).

113. Schroder, K., Zhou, R. \& Tschopp, J. The NLRP3 inflammasome: a sensor for metabolic danger? Science 327, 296-300 (2010).

114. Martinon, F., Pétrilli, V., Mayor, A., Tardivel, A. \& Tschopp, J. Goutassociated uric acid crystals activate the NALP3 inflammasome. Nature 440, 237-241 (2006).

115. Martinon, F., Gaide, O., Pétrilli, V., Mayor, A. \& Tschopp, J. NALP inflammasomes: a central role in innate immunity. Semin Immunopathol 29, 213-229 (2007).

116. Zare, F. et al. Uric acid, a nucleic acid degradation product, downregulates dsRNA-triggered arthritis. Journal of Leukocyte Biology 79, 482-488 (2006). 
117. Leighton, S., Kok, L.-F., Halliday, G. M. \& Byrne, S. N. Inhibition of UV-induced uric acid production using Allopurinol prevents suppression of the contact hypersensitivity response. Exp. Dermatol. 22, 189-194 (2013).

118. Serbina, N. V. \& Pamer, E. G. Monocyte emigration from bone marrow during bacterial infection requires signals mediated by chemokine receptor CCR2. Nat Immunol 7, 311-317 (2006).

119. Geissmann, F., Jung, S. \& Littman, D. R. Blood monocytes consist of two principal subsets with distinct migratory properties. Immunity 19, 71-82 (2003).

120. Cros, J. et al. Human CD14dim monocytes patrol and sense nucleic acids and viruses via TLR7 and TLR8 receptors. Immunity 33, 375386 (2010).

121. Swirski, F. K. et al. Identification of splenic reservoir monocytes and their deployment to inflammatory sites. Science 325, 612-616 (2009).

122. Martin, W. J., Shaw, O., Liu, X., Steiger, S. \& Harper, J. L. MSU crystal-recruited non-inflammatory monocytes differentiate into M1-like pro-inflammatory macrophages in a peritoneal murine model of gout. Arthritis Rheum (2011). doi:10.1002/art.30245

123. Roncal, C. A. et al. Effect of elevated serum uric acid on cisplatininduced acute renal failure. Am J Physiol Renal Physiol 292, F116-22 (2007).

124. Kang, D.-H. et al. A role for uric acid in the progression of renal disease. J Am Soc Nephrol 13, 2888-2897 (2002).

125. Schreiner, O., Wandel, E., Himmelsbach, F., Galle, P. R. \& MärkerHermann, E. Reduced secretion of proinflammatory cytokines of monosodium urate crystal-stimulated monocytes in chronic renal failure: an explanation for infrequent gout episodes in chronic renal failure patients? Nephrol Dial Transplant 15, 644-649 (2000).

126. Rodriguez-Iturbe, B., Vaziri, N. D., Herrera-Acosta, J. \& Johnson, R. J. Oxidative stress, renal infiltration of immune cells, and salt-sensitive hypertension: all for one and one for all. Am J Physiol Renal Physiol 286, F606-16 (2004).

127. Libby, P. Inflammation in atherosclerosis. Nature 420, 868-874 (2002).

128. Grainger, R., McLaughlin, R. J., Harrison, A. A. \& Harper, J. L. Hyperuricaemia elevates circulating CCL2 levels and primes monocyte trafficking in subjects with inter-critical gout. Rheumatology (Oxford) (2012). doi:10.1093/rheumatology/kes326

129. Kanellis, J. et al. Uric acid stimulates monocyte chemoattractant protein-1 production in vascular smooth muscle cells via mitogenactivated protein kinase and cyclooxygenase-2. Hypertension 41, 1287-1293 (2003).

130. Papathanassoglou, E. et al. Leptin receptor expression and signaling in lymphocytes: kinetics during lymphocyte activation, role in lymphocyte survival, and response to high fat diet in mice. J Immunol 176, 7745-7752 (2006).

131. Loffreda, S. et al. Leptin regulates proinflammatory immune responses. FASEB J 12, 57-65 (1998).

132. Rempel, J. D. et al. Preliminary analysis of immune activation in early onset type 2 diabetes. Int J Circumpolar Health 72, (2013). 
133. Shiny, A. et al. Convergence of innate immunity and insulin resistance as evidenced by increased nucleotide oligomerization domain (NOD) expression and signaling in monocytes from patients with type 2 diabetes. Cytokine (2013). doi:10.1016/j.cyto.2013.08.003

134. Jansen, H. J. et al. Start of insulin therapy in patients with type 2 diabetes mellitus promotes the influx of macrophages into subcutaneous adipose tissue. Diabetologia (2013). doi:10.1007/s00125-013-3018-6

135. Martin, W. J., Walton, M. \& Harper, J. Resident macrophages initiating and driving inflammation in a monosodium urate monohydrate crystalinduced murine peritoneal model of acute gout. Arthritis Rheum 60, 281-289 (2009).

136. Martin, W. J., Shaw, O., Liu, X., Steiger, S. \& Harper, J. L. Monosodium urate monohydrate crystal-recruited noninflammatory monocytes differentiate into M1-like proinflammatory macrophages in a peritoneal murine model of gout. Arthritis Rheum 63, 1322-1332 (2011).

137. Zhang, X. et al. Silkworms can be used as an animal model to screen and evaluate gouty therapeutic drugs. J. Insect Sci. 12, 4 (2012).

138. Simkin, P. A. The Dalmatian defect: a hepatic endocrinopathy of urate transport. Arthritis Rheum 52, 2257-2262 (2005).

139. Huang, C. G. et al. Hypouricemic effects of phenylpropanoid glycosides acteoside of Scrophularia ningpoensis on serum uric acid levels in potassium oxonate-pretreated Mice. Am J Chin Med 36, 149_ 157 (2008).

140. Wang, Y. et al. Administration of procyanidins from grape seeds reduces serum uric acid levels and decreases hepatic xanthine dehydrogenase/oxidase activities in oxonate-treated mice. Basic Clin Pharmacol Toxicol 94, 232-237 (2004).

141. Lo, H.-C., Wang, Y.-H., Chiou, H.-Y., Lai, S.-H. \& Yang, Y. Relative efficacy of casein or soya protein combined with palm or safflowerseed oil on hyperuricaemia in rats. Br J Nutr 104, 67-75 (2010).

142. Mazzali, M. et al. Elevated uric acid increases blood pressure in the rat by a novel crystal-independent mechanism. Hypertension 38, 1101-1106 (2001).

143. Sánchez-Lozada, L. G. et al. Treatment with the xanthine oxidase inhibitor febuxostat lowers uric acid and alleviates systemic and glomerular hypertension in experimental hyperuricaemia. Nephrol Dial Transplant 23, 1179-1185 (2008).

144. Scott, G. S. et al. Therapeutic intervention in experimental allergic encephalomyelitis by administration of uric acid precursors. Proc Natl Acad Sci USA 99, 16303-16308 (2002).

145. Wu, X. et al. Hyperuricemia and urate nephropathy in urate oxidasedeficient mice. Proc Natl Acad Sci USA 91, 742-746 (1994).

146. Preitner, F. et al. Glut9 is a major regulator of urate homeostasis and its genetic inactivation induces hyperuricosuria and urate nephropathy. Proc Natl Acad Sci USA 106, 15501-15506 (2009).

147. Kosugi, T. et al. Effect of lowering uric acid on renal disease in the type 2 diabetic db/db mice. Am J Physiol Renal Physiol 297, F481-8 (2009). 
148. Lanaspa, M. A. et al. Uric acid induces hepatic steatosis by generation of mitochondrial oxidative stress: potential role in fructose-dependent and -independent fatty liver. Journal of Biological Chemistry 287, 40732-40744 (2012).

149. Doshi, M., Takiue, Y., Saito, H. \& Hosoyamada, M. The increased protein level of URAT1 was observed in obesity/metabolic syndrome model mice. Nucleosides, Nucleotides \& Nucleic Acids 30, 1290-1294 (2011).

150. Chen, G.-L., Wei, W. \& Xu, S.-Y. Effect and mechanism of total saponin of Dioscorea on animal experimental hyperuricemia. Am J Chin Med 34, 77-85 (2006).

151. Lin, C.-C., Yin, M.-C., Hsu, C.-C. \& Lin, M.-P. Effect of five cysteinecontaining compounds on three lipogenic enzymes in Balb/cA mice consuming a high saturated fat diet. Lipids 39, 843-848 (2004).

152. Poudyal, H., Campbell, F. \& Brown, L. Olive leaf extract attenuates cardiac, hepatic, and metabolic changes in high carbohydrate-, high fat-fed rats. J. Nutr. 140, 946-953 (2010).

153. Nakagawa, T. A causal role for uric acid in fructose-induced metabolic syndrome. Am J Physiol Renal Physiol 290, F625-F631 (2005).

154. Sánchez-Lozada, L. G. et al. Effects of febuxostat on metabolic and renal alterations in rats with fructose-induced metabolic syndrome. Am J Physiol Renal Physiol 294, F710-8 (2008).

155. El-Bassossy, H. M., El-Fawal, R., Fahmy, A. \& Watson, M. L. Arginase inhibition alleviates hypertension in the metabolic syndrome. $\mathrm{Br} \mathrm{J}$ Pharmacol 169, 693-703 (2013).

156. Wallace, S. L. et al. Preliminary criteria for the classification of the acute arthritis of primary gout. Arthritis Rheum 20, 895-900 (1977).

157. Rahimian, $\mathrm{R}$. et al. Adenosine A2A receptors and uric acid mediate protective effects of inosine against TNBS-induced colitis in rats. Eur $J$ Pharmacol 649, 376-381 (2010).

158. Lowry, O. H., Rosebrough, N. J., Farr, A. L. \& Randall, R. J. Protein measurement with the Folin phenol reagent. J Biol Chem 193, 265275 (1951).

159. Berridge, M. V., Herst, P. M. \& Tan, A. S. Tetrazolium dyes as tools in cell biology: new insights into their cellular reduction. Biotechnol Annu Rev 11, 127-152 (2005).

160. Martin, W. J., Grainger, R., Harrison, A. \& Harper, J. L. Differences in MSU-induced superoxide responses by neutrophils from gout subjects compared to healthy controls and a role for environmental inflammatory cytokines and hyperuricemia in neutrophil function and survival. J. Rheumatol. 37, 1228-1235 (2010).

161. Kocaman, S. A. et al. The particular interactions of the traditional cardiovascular risk factors with different circulating specific leukocyte subtype counts in blood: an observational study. Anadolu Kardiyol Derg 11, 573-581 (2011).

162. Bordoni, V. et al. Protective effect of urate oxidase on uric acid induced-monocyte apoptosis. Curr Drug Discov Technol 2, 29-36 (2005).

163. Takeuchi, O. \& Akira, S. Toll-like receptors; their physiological role and signal transduction system. Int Immunopharmacol 1, 625-635 (2001). 
164. Spitsin, S. V., Scott, G. S., Kean, R. B., Mikheeva, T. \& Hooper, D. C. Protection of myelin basic protein immunized mice from free-radical mediated inflammatory cell invasion of the central nervous system by the natural peroxynitrite scavenger uric acid. Neurosci Lett 292, 137141 (2000).

165. Hooper, D. C. et al. Uric acid, a peroxynitrite scavenger, inhibits CNS inflammation, blood-CNS barrier permeability changes, and tissue damage in a mouse model of multiple sclerosis. FASEB J 14, 691-698 (2000).

166. Strasak, A. M. et al. The role of serum uric acid as an antioxidant protecting against cancer: prospective study in more than 28000 older Austrian women. Annals of Oncology 18, 1893-1897 (2007).

167. Naik, E. \& Dixit, V. M. Mitochondrial reactive oxygen species drive proinflammatory cytokine production. J Exp Med 208, 417-420 (2011).

168. Bulua, A. C. et al. Mitochondrial reactive oxygen species promote production of proinflammatory cytokines and are elevated in TNFR1associated periodic syndrome (TRAPS). J Exp Med 208, 519-533 (2011).

169. Rizvi, S. I., Zaid, M. A., Anis, R. \& Mishra, N. Protective role of tea catechins against oxidation-induced damage of type 2 diabetic erythrocytes. Clin. Exp. Pharmacol. Physiol. 32, 70-75 (2005).

170. Doyle, S. L. \& O'Neill, L. A. J. Toll-like receptors: From the discovery of NFKB to new insights into transcriptional regulations in innate immunity. Biochem Pharmacol 72, 1102-1113 (2006).

171. Cao, S., Zhang, X., Edwards, J. P. \& Mosser, D. M. NF-kappaB1 (p50) homodimers differentially regulate pro- and anti-inflammatory cytokines in macrophages. J Biol Chem 281, 26041-26050 (2006).

172. Beg, A. A., Finco, T. S., Nantermet, P. V. \& Baldwin, A. S. Tumor necrosis factor and interleukin-1 lead to phosphorylation and loss of I kappa B alpha: a mechanism for NF-kappa B activation. Molecular and Cellular Biology 13, 3301-3310 (1993).

173. Lang, R., Hammer, M. \& Mages, J. DUSP meet immunology: dual specificity MAPK phosphatases in control of the inflammatory response. J Immunol 177, 7497-7504 (2006).

174. Qian, F. et al. Map kinase phosphatase 5 protects against sepsisinduced acute lung injury. Am J Physiol Lung Cell Mol Physiol 302, L866-74 (2012).

175. Taxman, D. J. et al. The NLR adaptor ASC/PYCARD regulates DUSP10, mitogen-activated protein kinase (MAPK), and chemokine induction independent of the inflammasome. Journal of Biological Chemistry 286, 19605-19616 (2011).

176. Ippagunta, S. K. et al. The inflammasome adaptor ASC regulates the function of adaptive immune cells by controlling Dock2-mediated Rac activation and actin polymerization. Nat Immunol 12, 1010-1016 (2011).

177. Narayan, S., Kolly, L., So, A. \& Busso, N. Increased interleukin-10 production by ASC-deficient CD4+ T cells impairs bystander T-cell proliferation. Immunology 134, 33-40 (2011).

178. Takahashi, H. K. et al. Effects of adenosine on adhesion molecule expression and cytokine production in human PBMC depend on the 
receptor subtype activated. Br J Pharmacol 150, 816-822 (2007).

179. Link, A. A. et al. Ligand-activation of the adenosine A2a receptors inhibits IL-12 production by human monocytes. J Immunol 164, 436442 (2000).

180. Csóka, B. et al. A2A adenosine receptors and C/EBPbeta are crucially required for IL-10 production by macrophages exposed to Escherichia coli. Blood 110, 2685-2695 (2007).

181. Haskó, G. \& Cronstein, B. N. Adenosine: an endogenous regulator of innate immunity. Trends Immunol 25, 33-39 (2004).

182. Wei, C. J., Li, W. \& Chen, J. F. Normal and abnormal functions of adenosine receptors in the central nervous system revealed by genetic knockout studies. Biochim Biophys Acta 1808, 1358-1379 (2011).

183. Morelli, M., Carta, A. R., Kachroo, A. \& Schwarzschild, M. A. Pathophysiological roles for purines: adenosine, caffeine and urate. Prog. Brain Res. 183, 183-208 (2010).

184. Junger, W. G. Immune cell regulation by autocrine purinergic signalling. Nat Rev Immunol 11, 201-212 (2011).

185. Cronstein, G. H. A. B. Regulation of inflammation by adenosine. 1-8 (2013). doi:10.3389/fimmu.2013.00085/abstract

186. Linden, J. Molecular approach to adenosine receptors: receptormediated mechanisms of tissue protection. Annu. Rev. Pharmacol. Toxicol. 41, 775-787 (2001).

187. Feoktistov, I. \& Biaggioni, I. Adenosine A2b receptors evoke interleukin-8 secretion in human mast cells. An enprofylline-sensitive mechanism with implications for asthma. J Clin Invest 96, 1979-1986 (1995).

188. Mina-Osorio, P. CD13 in cell adhesion: aminopeptidase N (CD13) mediates homotypic aggregation of monocytic cells. Journal of Leukocyte Biology 79, 719-730 (2006).

189. Kirchberger, S., Vetr, H., Majdic, O., Stockinger, H. \& Stöckl, J. Engagement of ICAM-1 by major group rhinoviruses activates the LFA-1/ICAM-3 cell adhesion pathway in mononuclear phagocytes. Immunobiology 211, 537-547 (2006).

190. Davenpeck, K. L., Brummet, M. E., Hudson, S. A., Mayer, R. J. \& Bochner, B. S. Activation of human leukocytes reduces surface Pselectin glycoprotein ligand-1 (PSGL-1, CD162) and adhesion to Pselectin in vitro. $\mathrm{J}$ Immunol 165, 2764-2772 (2000).

191. Tedder, T. F., Steeber, D. A., Chen, A. \& Engel, P. The selectins: vascular adhesion molecules. FASEB J 9, 866-873 (1995).

192. Muller, W. A. Mechanisms of leukocyte transendothelial migration. Annu. Rev. Pathol. Mech. Dis. 6, 323-344 (2011).

193. Schulthess, J. et al. Interleukin-15-Dependent NKp46(+) Innate Lymphoid Cells Control Intestinal Inflammation by Recruiting Inflammatory Monocytes. Immunity (2012). doi:10.1016/j.immuni.2012.05.013

194. Ingersoll, M. A., Platt, A. M., Potteaux, S. \& Randolph, G. J. Monocyte trafficking in acute and chronic inflammation. Trends Immunol 32, 470-477 (2011).

195. Hu, Q.-H., Jiao, R.-Q., Wang, X., Lv, Y.-Z. \& Kong, L.-D. Simiao pill 
ameliorates urate underexcretion and renal dysfunction in hyperuricemic mice. J Ethnopharmacol 128, 685-692 (2010).

196. So, A. \& Thorens, B. Uric acid transport and disease. J Clin Invest 120, 1791-1799 (2010).

197. Mughal, T. I., Ejaz, A. A., Foringer, J. R. \& Coiffier, B. An integrated clinical approach for the identification, prevention, and treatment of tumor lysis syndrome. Cancer Treat. Rev. 36, 164-176 (2010).

198. Maiguel, D. et al. Small molecule-mediated activation of the integrin CD11b/CD18 reduces inflammatory disease. Science Signaling 4, ra57 (2011).

199. Arend, W. P., Joslin, F. G., Thompson, R. C. \& Hannum, C. H. An IL-1 inhibitor from human monocytes. Production and characterization of biologic properties. J Immunol 143, 1851-1858 (1989).

200. Hammer, M. et al. Dual specificity phosphatase 1 (DUSP1) regulates a subset of LPS-induced genes and protects mice from lethal endotoxin shock. J Exp Med 203, 15-20 (2006).

201. Riemann, M., Endres, R., Liptay, S., Pfeffer, K. \& Schmid, R. M. The IkappaB protein $\mathrm{Bcl}-3$ negatively regulates transcription of the IL-10 gene in macrophages. J Immunol 175, 3560-3568 (2005).

202. Hayden, M. S. \& Ghosh, S. Shared principles in NF-kappaB signaling. Cell 132, 344-362 (2008).

203. Mimnaugh, E. G., Bonvini, P. \& Neckers, L. The measurement of ubiquitin and ubiquitinated proteins. Electrophoresis 20, 418-428 (1999).

204. Shi, C.-S. et al. Activation of autophagy by inflammatory signals limits IL-1 $\beta$ production by targeting ubiquitinated inflammasomes for destruction. Nat Immunol 13, 255-263 (2012).

205. Matunis, M. J., Coutavas, E. \& Blobel, G. A novel ubiquitin-like modification modulates the partitioning of the Ran-GTPase-activating protein RanGAP1 between the cytosol and the nuclear pore complex. J. Cell Biol. 135, 1457-1470 (1996).

206. Geiss-Friedlander, R. \& Melchior, F. Concepts in sumoylation: a decade on. Nat Rev Mol Cell Biol 8, 947-956 (2007).

207. Gibbings, S. et al. Xanthine oxidoreductase promotes the inflammatory state of mononuclear phagocytes through effects on chemokine expression, peroxisome proliferator-activated receptor\{gamma\} sumoylation, and HIF-1\{alpha\}. J Biol Chem 286, 961-975 (2011).

208. Walker, G. et al. 3-deazaadenosine prevents adhesion molecule expression and atherosclerotic lesion formation in the aortas of C57BL/6J mice. Arterioscler Thromb Vasc Biol 19, 2673-2679 (1999).

209. Tamura, R. et al. Effect of beta2-adrenergic receptor agonists on intercellular adhesion molecule (ICAM)-1, B7, and CD40 expression in mixed lymphocyte reaction. Transplantation 77, 293-301 (2004).

210. Wasilewska, A., Tenderenda, E., Taranta-Janusz, K., Tobolczyk, J. \& Stypułkowska, J. Markers of systemic inflammation in children with hyperuricemia. Acta Paediatr 101, 497-500 (2012).

211. Kobayashi, T., Kouzaki, H. \& Kita, H. Human eosinophils recognize endogenous danger signal crystalline uric acid and produce proinflammatory cytokines mediated by autocrine ATP. The Journal of 
Immunology 184, 6350-6358 (2010).

212. Ryan, K. A., Smith, M. F., Sanders, M. K. \& Ernst, P. B. Reactive oxygen and nitrogen species differentially regulate Toll-like receptor 4mediated activation of NF-kappa B and interleukin-8 expression. Infect. Immun. 72, 2123-2130 (2004).

213. Kahn, K. \& Tipton, P. A. Spectroscopic characterization of intermediates in the urate oxidase reaction. Biochemistry $37,11651-$ 11659 (1998).

214. Akaike, T. et al. Dependence on O2- generation by xanthine oxidase of pathogenesis of influenza virus infection in mice. $J$ Clin Invest 85, 739-745 (1990).

215. Boomer, J. S. et al. Immunosuppression in patients who die of sepsis and multiple organ failure. JAMA 306, 2594-2605 (2011).

216. Sen, A. \& Yende, S. Towards personalized medicine in sepsis: Quest for Shangri-La? Crit Care 17, 303 (2013).

217. Chuang, C.-C. et al. Serum total antioxidant capacity reflects severity of illness in patients with severe sepsis. Crit Care 10, R36 (2006).

218. Barnett, R. E. et al. Poor outcome in bacterial peritonitis is associated with dysregulated microRNAs and an increased inflammatory response. Surgery 154, 521-527 (2013).

219. Netea, M. G., Kullberg, B. J., Blok, W. L., Netea, R. T. \& van der Meer, $\mathrm{J}$. W. The role of hyperuricemia in the increased cytokine production after lipopolysaccharide challenge in neutropenic mice. Blood 89, 577-582 (1997).

220. Feig, J. E. et al. Regression of atherosclerosis is characterized by broad changes in the plaque macrophage transcriptome. PLoS ONE 7, e39790 (2012).

221. Ferrante, A. W. The immune cells in adipose tissue. Diabetes Obes Metab 15 Suppl 3, 34-38 (2013).

222. Kono, H. \& Rock, K. L. How dying cells alert the immune system to danger. Nat Rev Immunol 8, 279-289 (2008).

223. Kool, M. et al. Alum adjuvant boosts adaptive immunity by inducing uric acid and activating inflammatory dendritic cells. $J$ Exp Med 205, 869-882 (2008).

224. Ippagunta, S. K. et al. Inflammasome-independent Role of Apoptosisassociated Speck-like Protein Containing a CARD (ASC) in T Cell Priming Is Critical for Collagen-induced Arthritis. Journal of Biological Chemistry 285, 12454-12462 (2010). 


\section{Appendices}

\subsection{NFKB signalling in human monocytes following in vitro UA treatment}

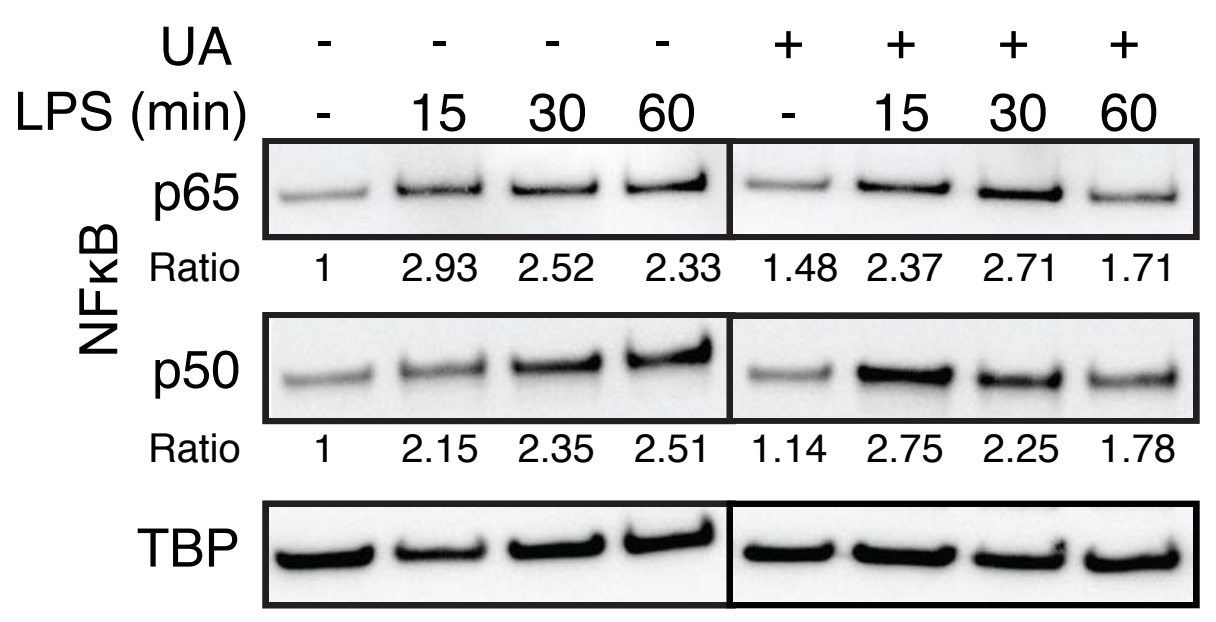

Figure 7.1 NFKB p65 nuclear translocation is inhibited and p50 in enhanced by soluble UA.

Western blot of nuclear NFKB p65 and p50 subunits from human blood monocytes pre-treated with or without UA $(0.6 \mathrm{mM})$ for $2 \mathrm{hr}$ and 15,30 or $60 \mathrm{~min}$ with LPS $(100 \mathrm{ng} / \mathrm{ml})$. Tata binding protein (TBP) was used as a loading control. Monocyte western blots were analysed with ImageJ software to determine band density and normalised to loading controls (TBP) and unstimulated control (lane1). Results represent three independent experiments from individual volunteers. 
7.2 MAP kinase signalling in human monocytes following in vitro UA treatment

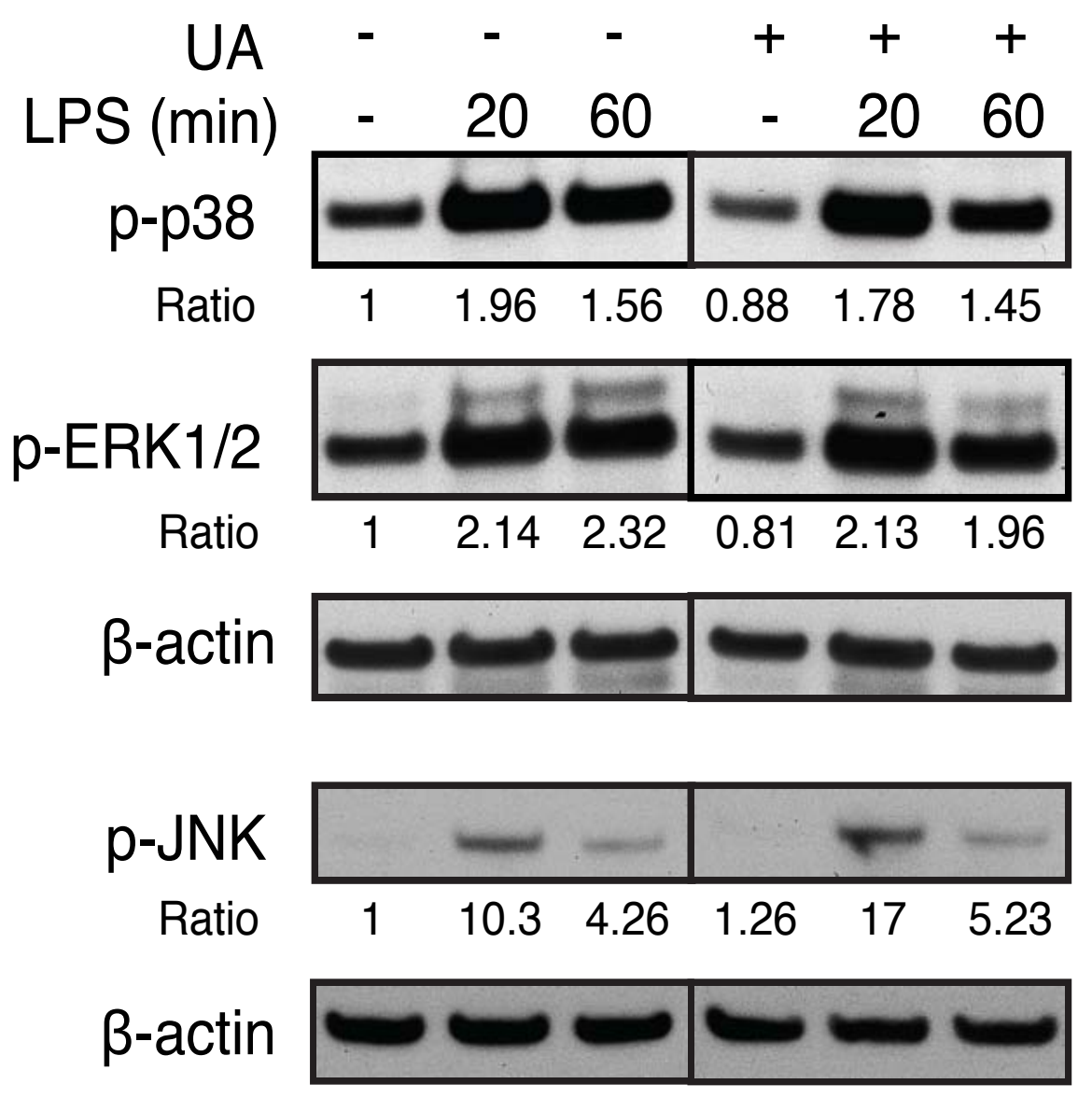

Figure 7.2 p38 and ERK1/2 MAP kinases are more rapidly dephosphorylated in UA treated monocytes.

Western blot of cytosolic MAP kinase phosphorylation from human blood monocytes pre-treated with or without UA (0.6mM) for $2 \mathrm{hr}$ and 20 or $60 \mathrm{~min}$ with LPS $(100 \mathrm{ng} / \mathrm{ml})$. $\beta$-actin was used as a loading control.Monocyte western blots were analysed with ImageJ software to determine band density and normalised to loading controls ( $\beta$ actin) and unstimulated control (lane1). Results represent three independent experiments from individual volunteers. 
7.3 Densitometric analysis of western blot data from figure 4.5 and 4.6
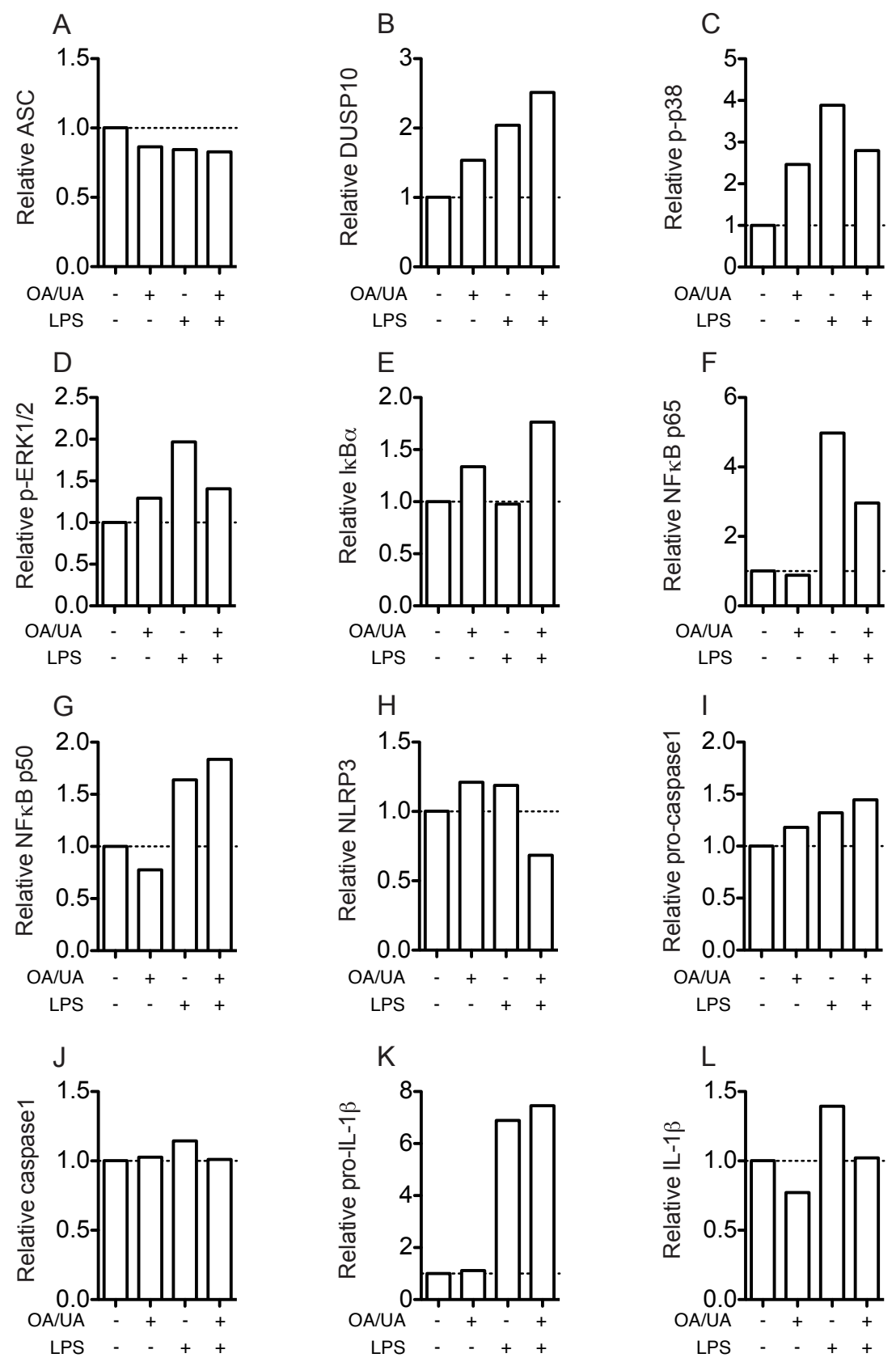

Figure 7.3 Densitometric analysis of western blot data from figure 4.5 and 4.6.

A-E and H-L: Cytosolic and F-G: nuclear protein from purified splenic monocytes isolated from C57 mice $(n=5)$ pre-treated with or without oxonic acid $(O A 300 \mathrm{mg} / \mathrm{kg}$, $30 \mathrm{~min}$ ) and UA (12ug/kg, $1 \mathrm{hr})$, then administered LPS i.v. (40ng/kg, $1 \mathrm{hr})$. Protein was analysed by western blotting. Results are representative of two independent experiments. Monocyte western blots were analysed with ImageJ software to determine band density and normalised to loading controls ( $\beta$-actin) and unstimulated control (lane1). 


\subsection{Densitometric analysis of western blot data from figure 4.8}
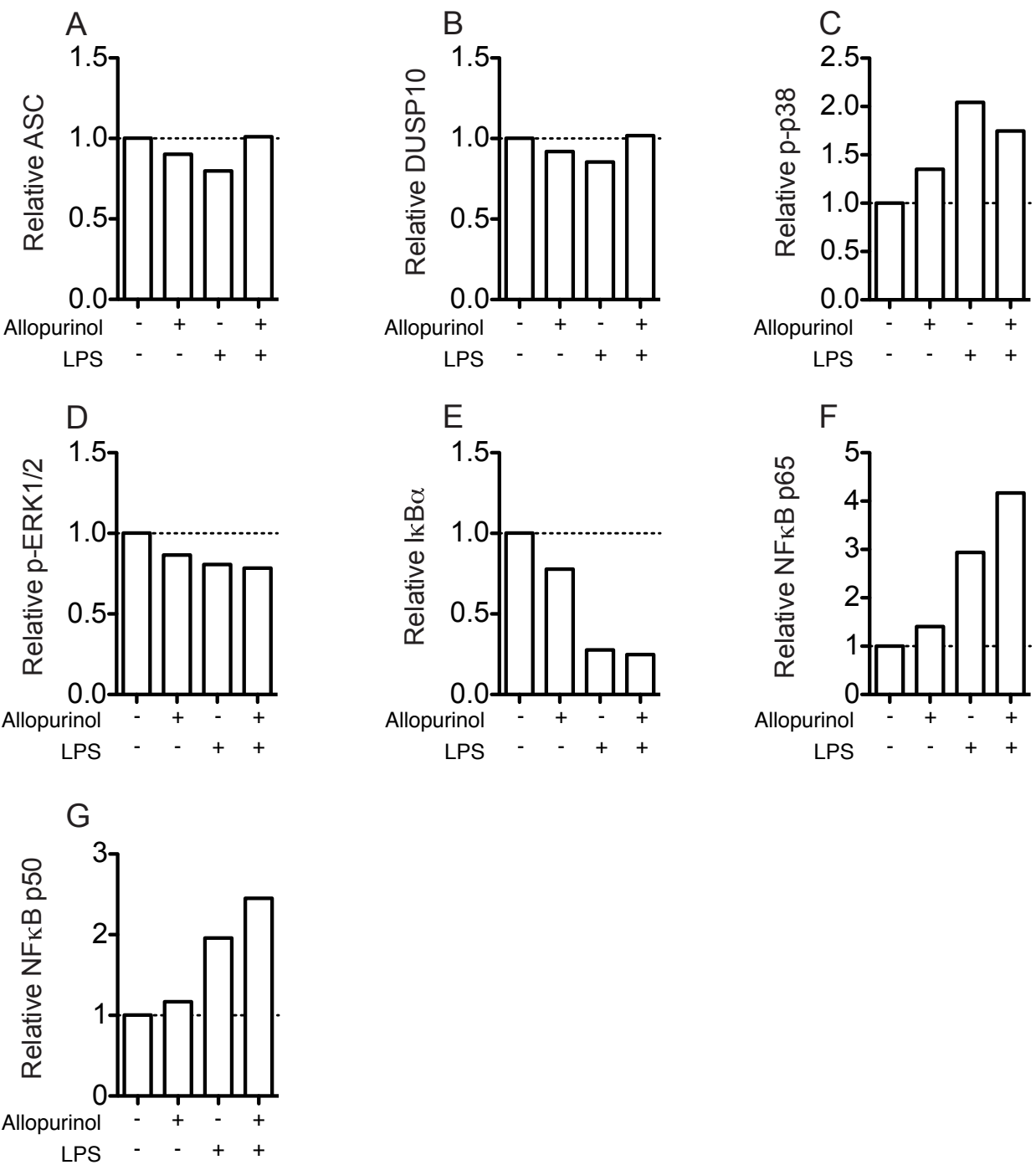

Figure 7.4 Densitometric analysis of western blot data from figure 4.8.

A-E: Cytosolic and F-G: nuclear protein from purified splenic monocytes isolated from C57 mice $(n=5)$ pre-treated with or without allopurinol $(30 \mathrm{mg} / \mathrm{kg}$, $3 \mathrm{hr})$ then administered LPS i.v. (40ng/kg, $1 \mathrm{hr}$ ). Protein was analysed by western blotting. Results are representative of two independent experiments. Monocyte western blots were analysed with ImageJ software to determine band density and normalised to loading controls ( $\beta$-actin) and unstimulated control (lane1). 


\subsection{Densitometric analysis of western blot data from figure 4.10}
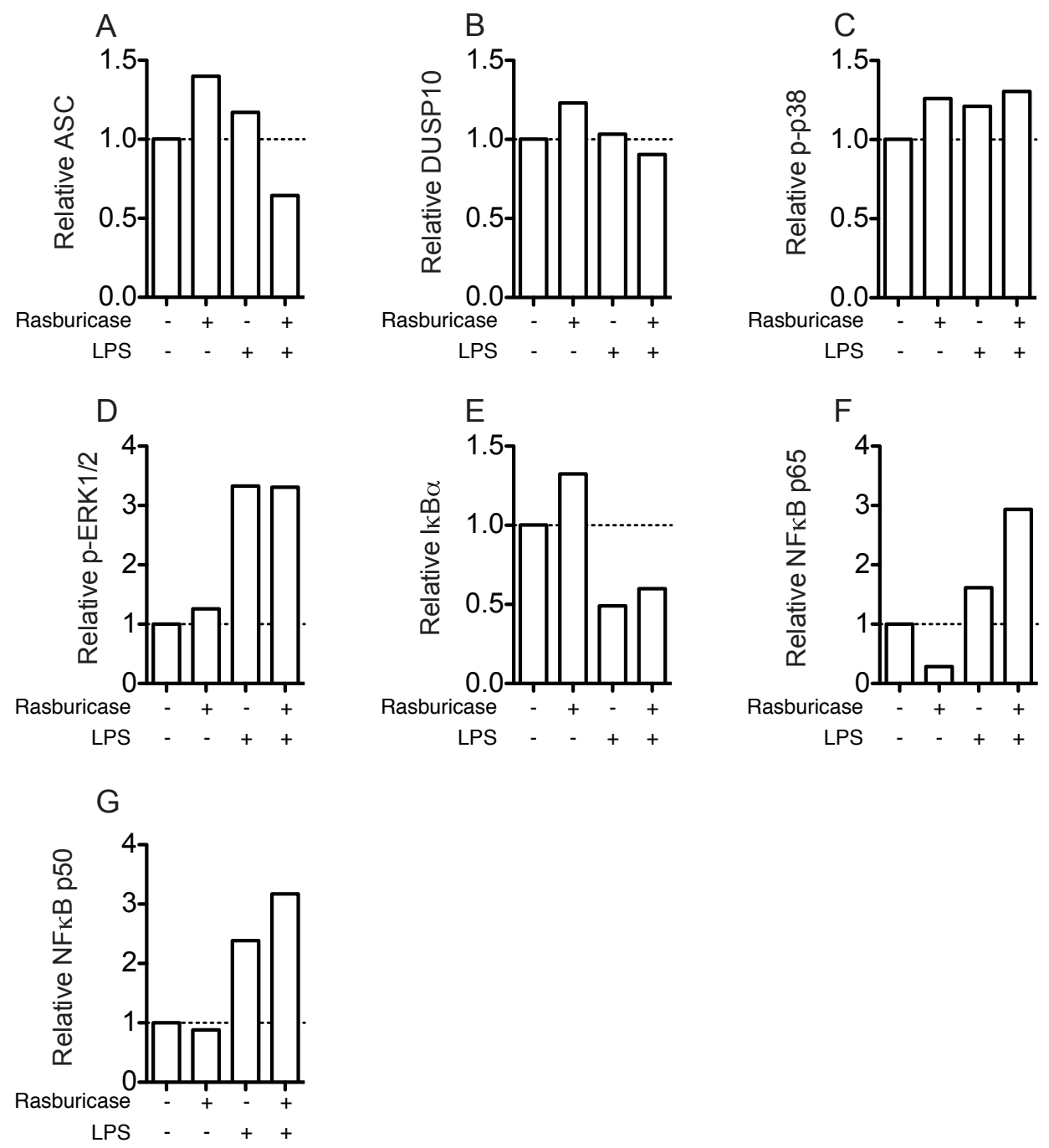

Figure 7.5 Densitometric analysis of western blot data from figure 4.10.

A-E: Cytosolic and F-G: nuclear protein from purified splenic monocytes isolated from C57 mice $(n=5)$ pre-treated with or without rasburicase $(0.8 \mathrm{mg} / \mathrm{kg}$, 3hr) then administered LPS i.v. (40ng/kg, 1hr). Protein was analysed by western blotting. Results are representative of two independent experiments. Monocyte western blots were analysed with ImageJ software to determine band density and normalised to loading controls ( $\beta$-actin) and unstimulated control (lane1). 


\subsection{Densitometric analysis of western blot data from figure 4.11}
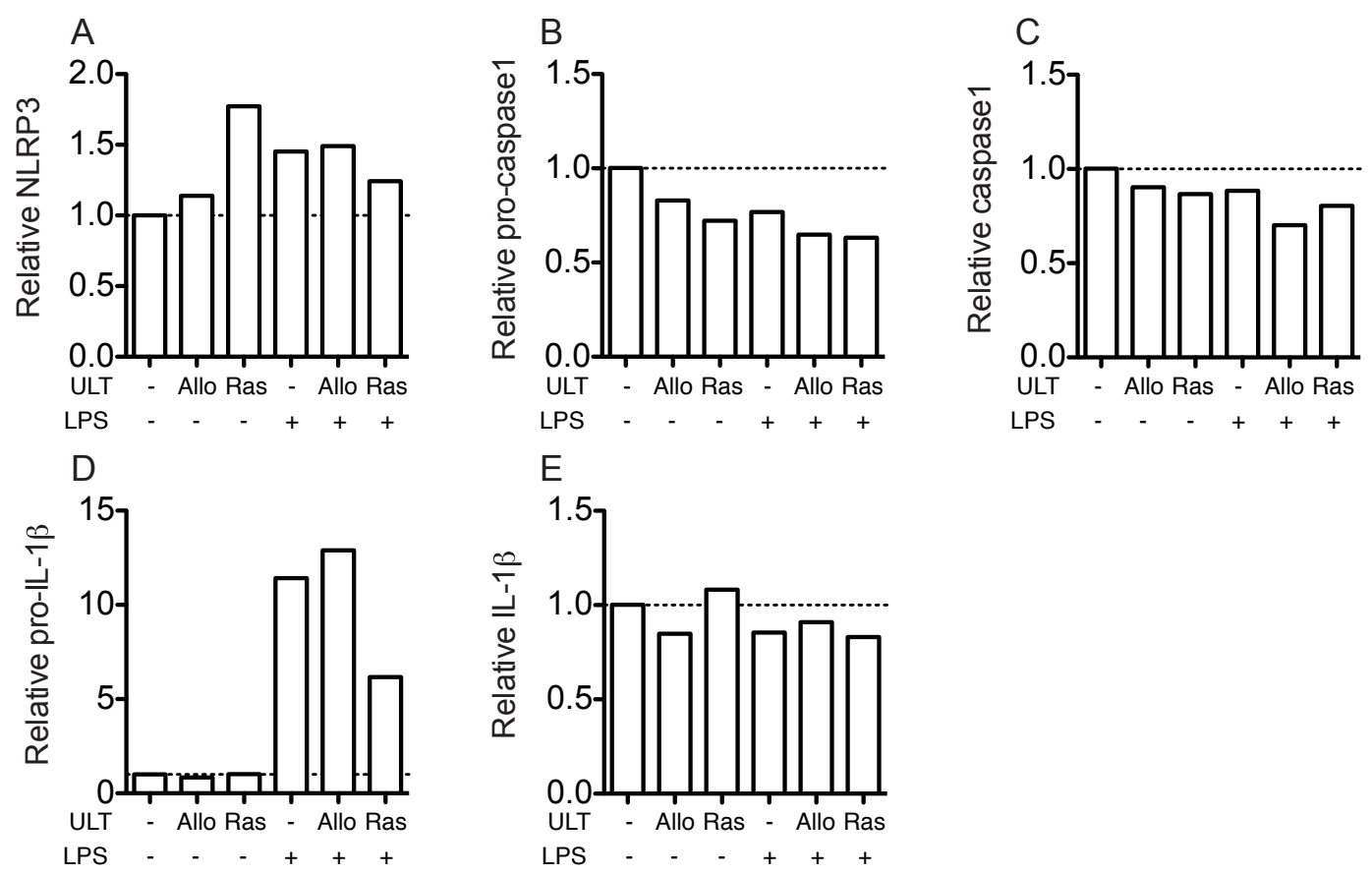

Figure 7.6 Densitometric analysis of western blot data from figure 4.11.

Cytosolic protein from purified splenic monocytes isolated from C57 mice $(n=5)$ pretreated with or without allopurinol $(30 \mathrm{mg} / \mathrm{kg}$, 3hr) or rasburicase $(0.8 \mathrm{mg} / \mathrm{kg}$, 3hr) then administered LPS i.v. (40ng/kg, 1hr). Protein was analysed by western blotting. Results are representative of two independent experiments. Monocyte western blots were analysed with ImageJ software to determine band density and normalised to loading controls ( $\beta$-actin) and unstimulated control (lane1). 
7.7 Densitometric analysis of western blot data from figure 4.14 and 4.15
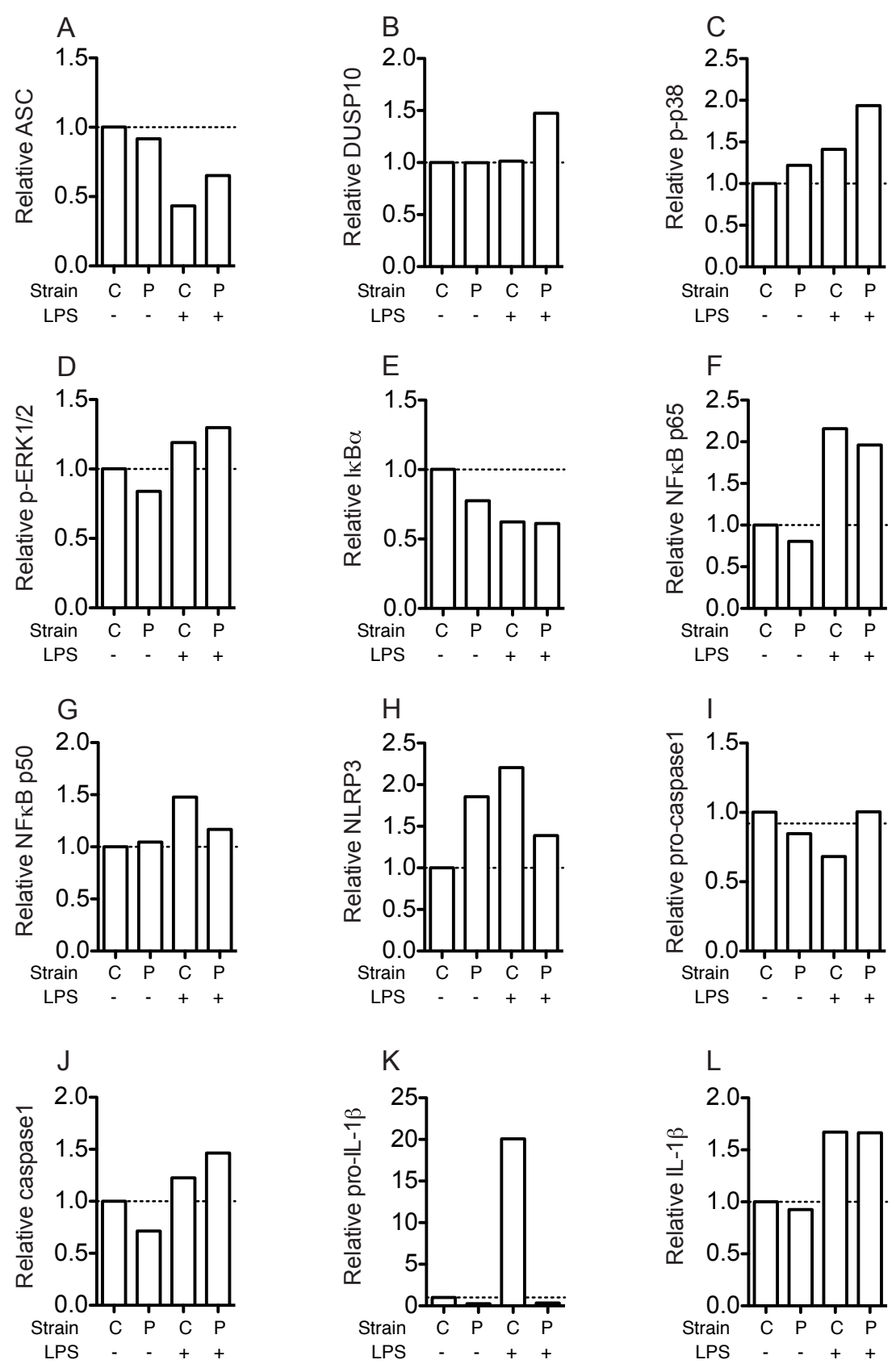

Figure 7.7 Densitometric analysis of western blot data from figure 4.14 and 4.15.

A-E and H-L: Cytosolic and F-G: nuclear protein from purified splenic monocytes isolated from C57 and PLT2 mice ( $n=5)$ administered LPS i.v. (40ng/kg, 1hr). Protein was analysed by western blotting. Strain: $C=C 57$ and $P=P L T 2$. Results are representative of two independent experiments. Monocyte western blots were analysed with ImageJ software to determine band density and normalised to loading controls ( $\beta$-actin) and unstimulated control (lane1). 


\subsection{Densitometric analysis of western blot data from figure 4.17} and 4.18
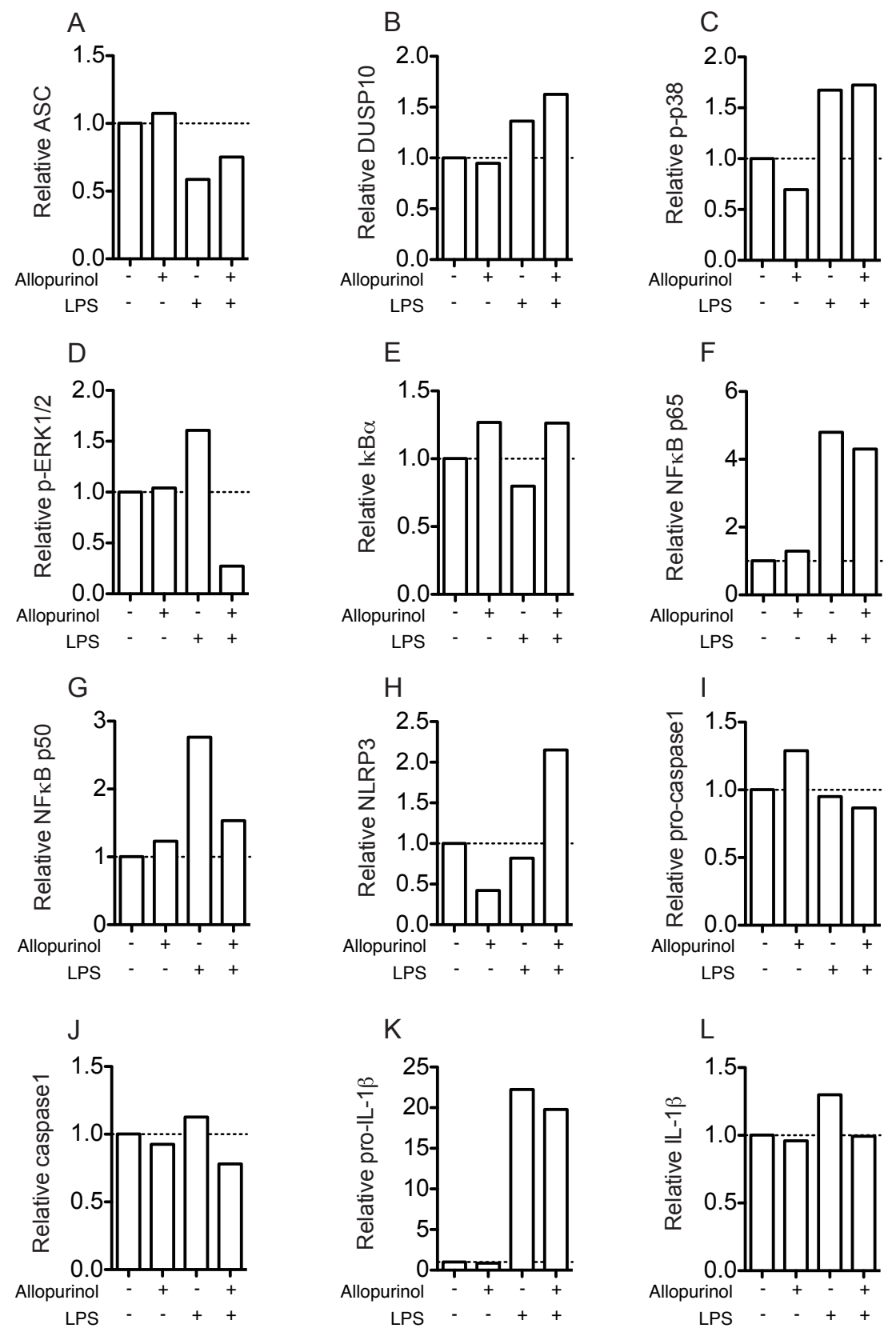

Figure 7.8 Densitometric analysis of western blot data from figure 4.17 and 4.18.

A-E and H-L: Cytosolic and F-G: nuclear protein from purified splenic monocytes isolated from PLT2 mice ( $\mathrm{n=5}$ ) pre-treated with or without allopurinol $(30 \mathrm{mg} / \mathrm{kg}$, 3hr) then administered LPS i.v. (40ng/kg, $1 \mathrm{hr})$. Protein was analysed by western blotting. Results are representative of two independent experiments. Monocyte western blots were analysed with ImageJ software to determine band density and normalised to loading controls ( $\beta$-actin) and unstimulated control (lane1). 


\subsection{Densitometric analysis of western blot data from figure 4.20} and 4.21
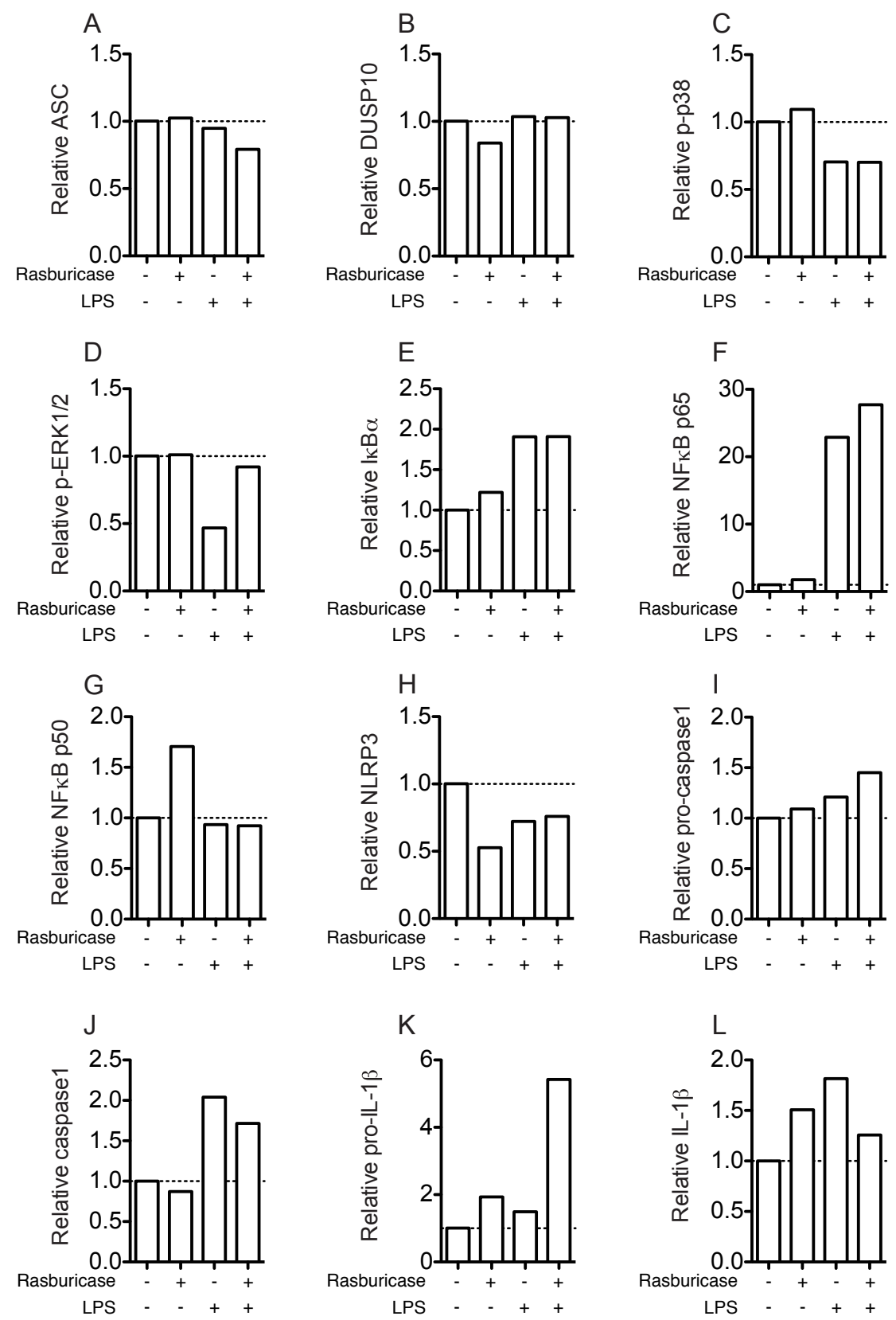

Figure 7.9 Densitometric analysis of western blot data from figure 4.20 and 4.21.

A-E and H-L: Cytosolic and F-G: nuclear protein from purified splenic monocytes isolated from PLT2 mice $(n=5)$ pre-treated with or without rasburicase $10.8 \mathrm{mg} / \mathrm{kg}$, $3 \mathrm{hr}$ ) then administered LPS i.v. (40ng/kg, $1 \mathrm{hr}$ ). Protein was analysed by western blotting. Results are representative of two independent experiments. Monocyte western blots were analysed with ImageJ software to determine band density and normalised to loading controls ( $\beta$-actin) and unstimulated control (lane1). 


\subsection{Serum cytokines and chemokines during ULT}

\section{Serum Cytokines}

A

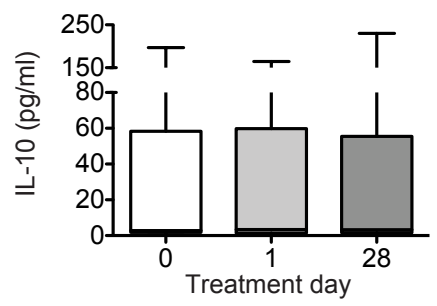

C

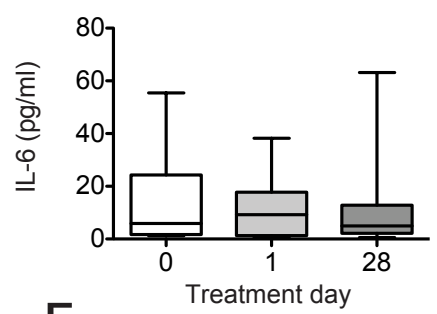

E

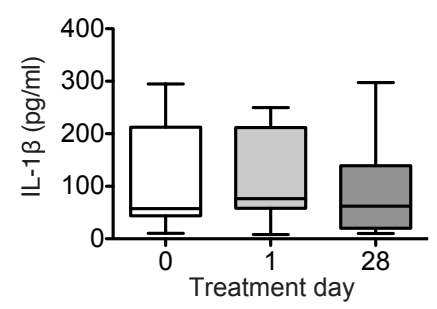

B

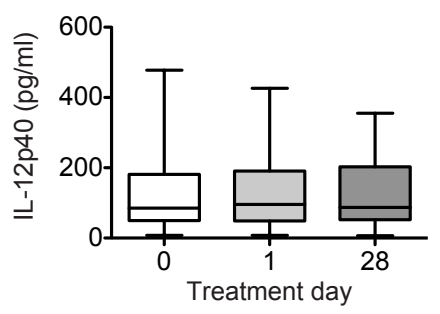

$\mathrm{D}$

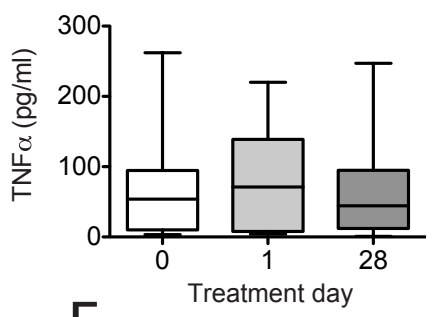

F

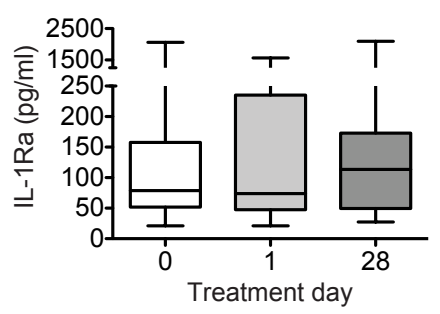

Serum Chemokines

$G$
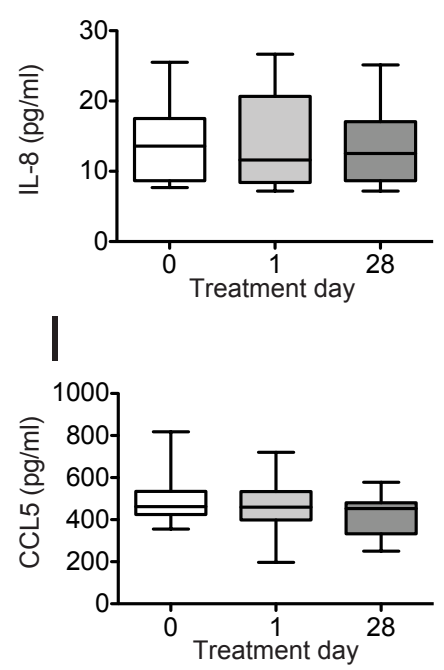
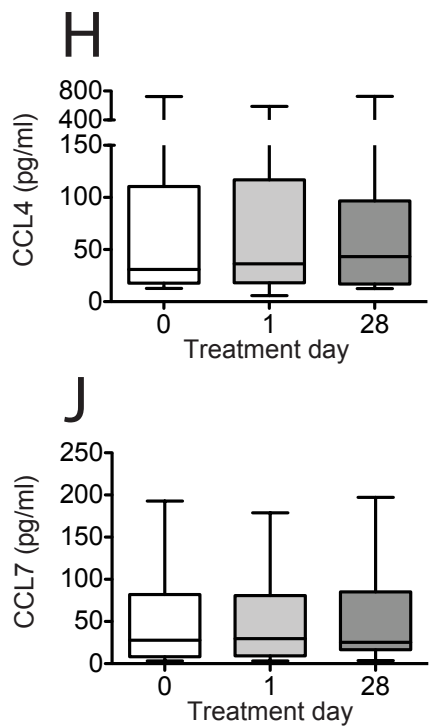

Figure 7.10 ULT does not alter human serum cytokine or chemokine levels.

Cytokines and chemokines in human serum from day 0 , day 1 and day 28 of ULT were analysed by multiplex bead array. A: IL-10, B: IL12p40, C: IL-6, D: TNF $\alpha$, E: IL1ß, F: IL-1Ra, G: IL-8, H: CCL4, I: CCL5, J: CCL7. 


\subsection{Serum cytokines and chemokines versus serum UA levels}

Serum Cytokines

A
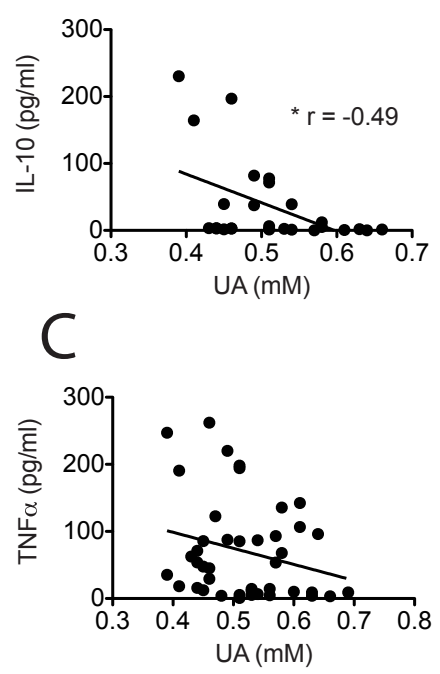

E

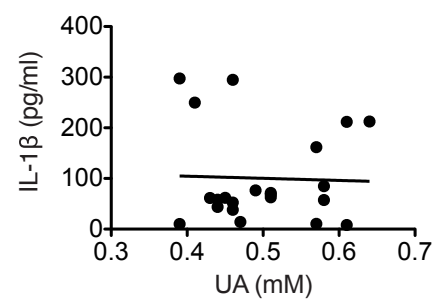

B

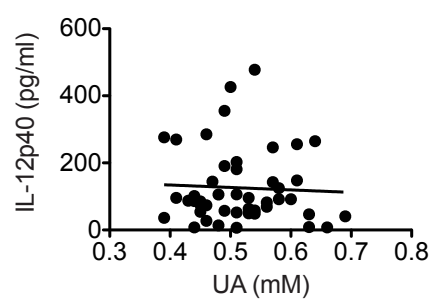

D

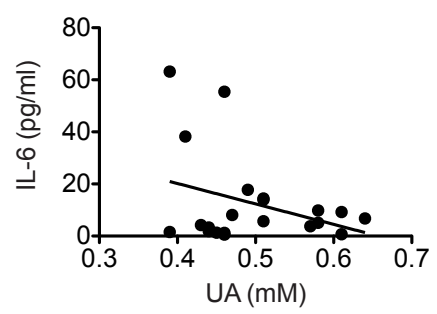

$\mathrm{F}$

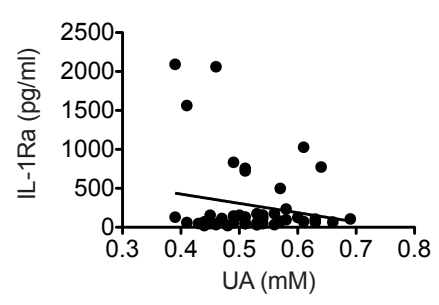

Serum Chemokines

G
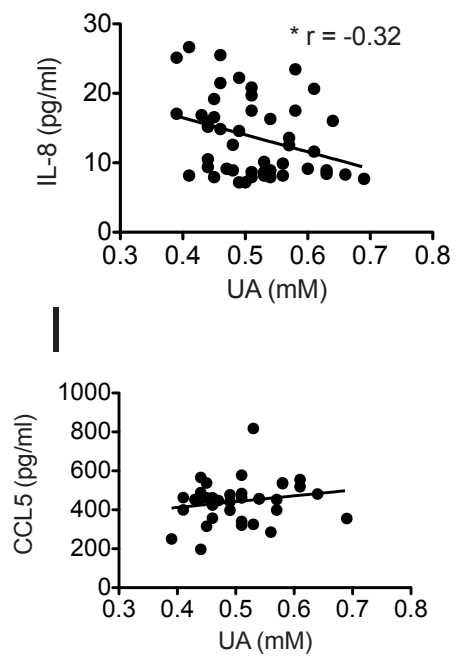

$\mathrm{H}$
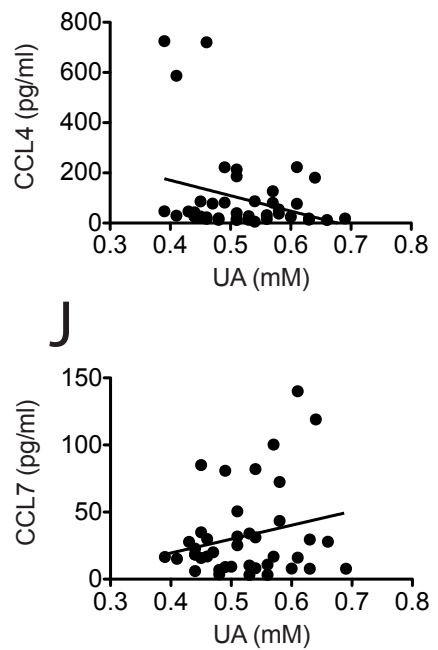

Figure 7.11 Serum IL-10 and IL-8 levels negatively correlate with serum UA. Cytokines and chemokines in human serum from day 0 , day 1 and day 28 of ULT were analysed by multiplex bead array. A: IL-10, B: IL12p40, C: IL-6, D: TNF $\alpha$, E: IL13, F: IL-1Ra, G: IL-8, H: CCL4, I: CCL5, J: CCL7. * ${ }^{*}<0.05$, Pearson's correlation coefficient. 


\subsection{Clinical western blots of LPS treated monocytes}
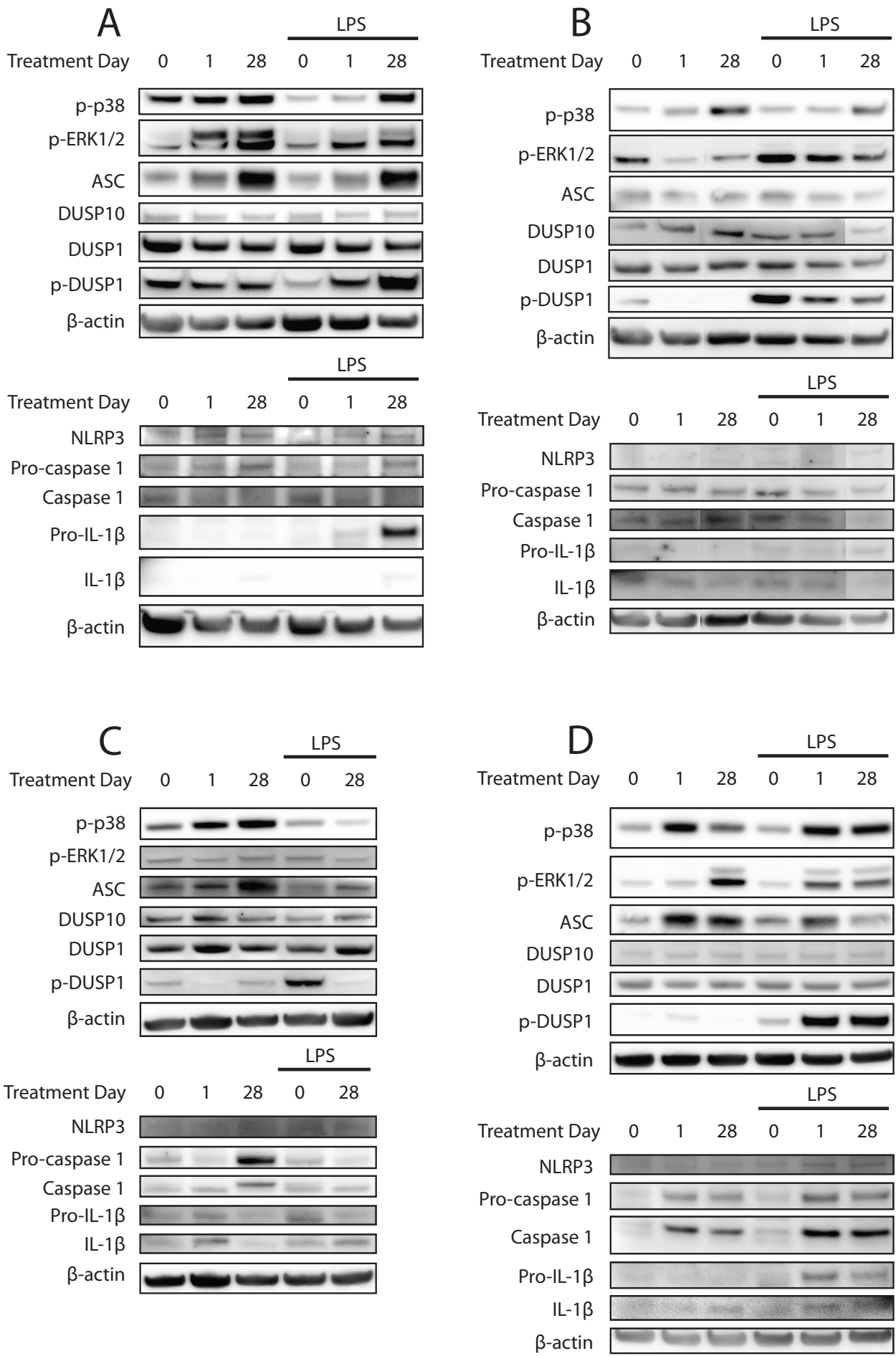

Figure 7.12 Western blots of LPS treated monocyte cytosolic protein from patients undergoing ULT.

Cytosolic protein was isolated from blood monocytes purified from patients before (0) and after 1 and 28 days of ULT. Monocytes were unstimulated $(n=8)$ or stimulated for $1 \mathrm{hr}$ with A: LPS $(100 \mathrm{ng} / \mathrm{ml} \mathrm{n=4})$ or $B:$ MSU $(0.5 \mathrm{mg} / \mathrm{ml} \mathrm{n}=4)$. Protein extracts were analysed by western blotting. $A, B, C$ and $D$ correspond with patient experimental numbers 7, 10, 12 and 14 respectively. 


\subsection{Clinical western blots of MSU treated monocytes}
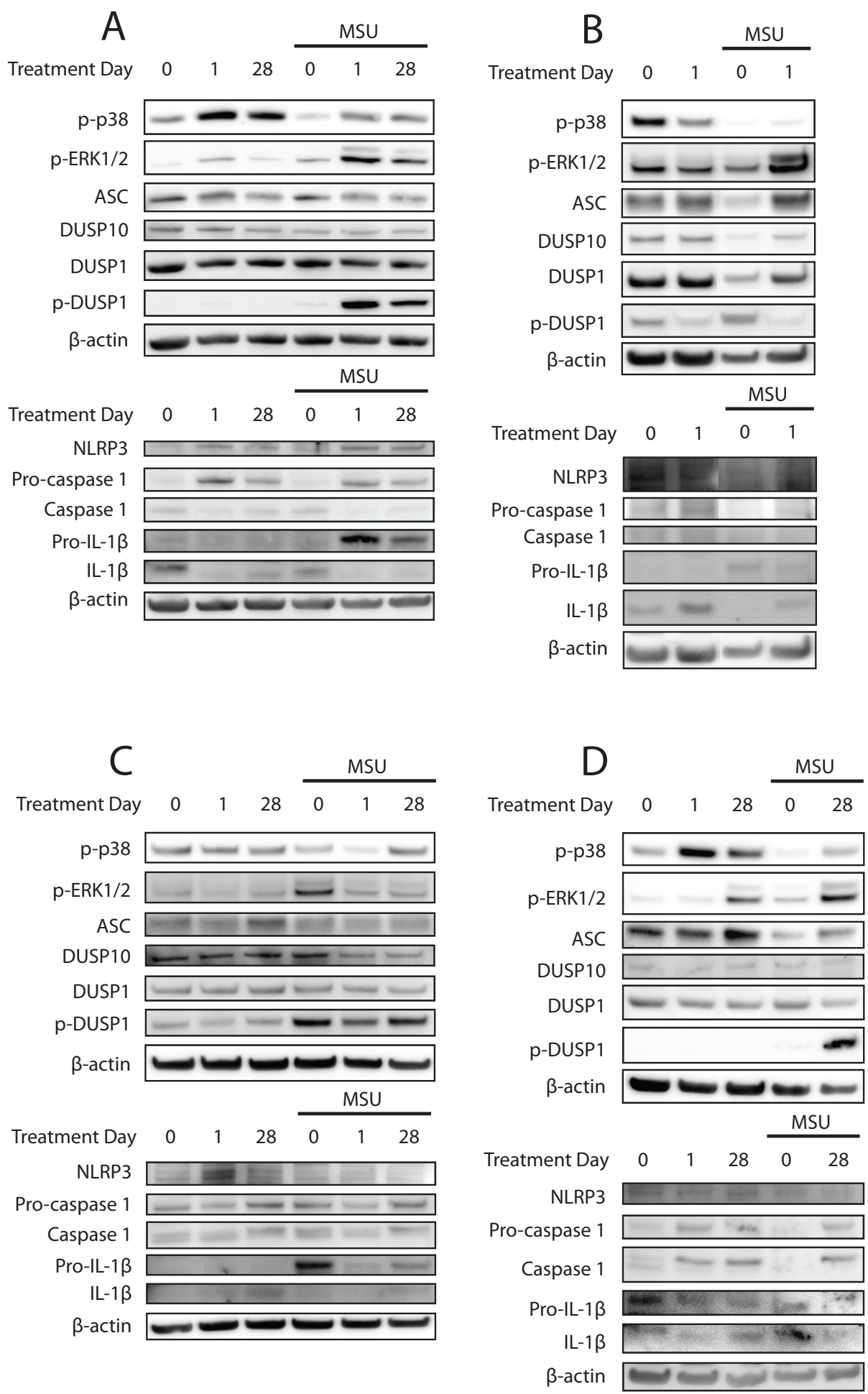

Figure 7.13 Western blots of MSU treated monocyte cytosolic protein from patients undergoing ULT. Cytosolic protein was isolated from blood monocytes purified from patients before (0) and after 1 and 28 days of ULT. Monocytes were unstimulated $(n=8)$ or stimulated for $1 \mathrm{hr}$ with $A$ : LPS $(100 \mathrm{ng} / \mathrm{ml} n=4)$ or $B$ : MSU $(0.5 \mathrm{mg} / \mathrm{ml} \mathrm{n}=4)$. Protein extracts were analysed by western blotting. $A, B, C$ and $D$ correspond with patient experimental numbers $9,11,13$ and 15 respectively. 